\title{
Emerging role of RNA-binding proteins in sporadic and rapid progressive Alzheimer's disease
}

\author{
Dissertation \\ for the award of the degree \\ “Doctor rerum naturalium" (Dr. rer. nat.) \\ of the Georg-August-Universität Göttingen \\ within the doctoral program "Molecular Medicine" \\ of the Georg-August University School of Science (GAUSS)
}

\author{
Submitted by \\ NEELAM YOUNAS \\ from Sialkot, Pakistan
}

Göttingen, 2019 


\section{Thesis Committee}

Prof. Dr. med. Inga Zerr (supervisor)

Prion research group, Department of Neurology, UMG, Göttingen

Prof. Dr. Thomas Meyer (first member of the thesis committee)

Department of Psychosomatic Medicine and Psychotherapy, UMG, Göttingen

Prof. Dr. Tiago Fleming Outeiro (second member of the thesis committee)

Department of Experimental Neurodegeneration, UMG, Göttingen

\section{Members of the Examination Board}

\section{Prof. E. A. Wimmer}

Department of Developmental Biology, Göttingen Center for Molecular Biosciences, Göttingen

\section{Prof. Dr. Stefan Jacobs}

Department of NanoBiophotonics, Max Planck Institute for Biophysical Chemistry, Göttingen

PD Dr. Michael Hoppert

Department of General Microbiology, Georg August University Göttingen

\section{Dr. Dieter Klopfenstein}

Department of Biophysics, University of Göttingen, Göttingen

Date of oral examination: 14-01-2020 


\section{DECLARATION}

I hereby declare that the Ph.D. thesis entitled "Emerging role of RNA-binding proteins in sporadic and rapid progressive Alzheimer's disease" is exclusively my own work. It is written solely by me and it does not contain any already published / written material except quoted with references

Neelam Younas

Göttingen, September 30th 2019 
Dedicated to

My wonderful and loving sister: Swaiba Younas 
"Every stress leaves an indelible scar, and the organism pays for its survival after a stressful situation by becoming a little older"

(Selye, 1950) 


\section{Table of Contents}

Abbreviations__ 1

Summary___ 4

1 Introduction__ 7

1.1 RNA-binding proteins (RBPs)

1.2 Alzheimer's disease ___ 13

1.3 Atypical subtype of Alzheimer's disease __ 18

2 Materials and methods __ 21

$2.1 \quad$ Materials

2.1.1 Antibodies _ 21

2.1.2 Antibiotics, enzymes and standards___ 22

2.1.3 Bacterial strain and culture media___ 22

2.1.4 Cell culture reagents

2.1.5 Chemicals__ 22

2.1.6 Instruments and other materials _ 23

2.1.7 Kits 24

2.1.8 Mammalian cell lines and culture media

2.1.9 Plasmids__ 24

2.1.10 Primer pairs _ 24

2.1.11 Software and online tools ___ 25

2.1.12 Solutions and buffers 25

2.2 Methods 227

2.2.1 Patient cohorts and sample processing __ 27

2.2.1.1 Pathological profiles__ 28

2.2.2 Molecular biology methods __ 29

2.2.2.1 Genomic DNA extraction __ 29

2.2.2.2 APOE genotyping _ 29

2.2.2.3 RNA extraction _ 30

2.2.2.4 Tissue lysis for pull-down assay __ 30 


\section{Contents}

2.2.2.5 RNA pull-down assay 31

2.2.2.6 Label-free quantification mass spectrometry (LFQ-MS) analysis 31

2.2.2.7 Differential enrichment analysis of RBPome 33

2.2.2.8 Quantitative real-time PCR (qRT-PCR)__ 34

2.2.2.9 Preparation of brain homogenates for protein analysis__ 34

2.2.2.10 Immunoblotting___ 35

2.2.2.11 Immunohistochemistry ___ 35

2.2.2.12 Confocal laser scanning and image analysis___ 36

2.2.3 Microbiological methods __ 36

2.2.3.1 Culturing and storage of E. coli _ 36

2.2.3.2 Extraction of plasmid DNA ___ 37

2.2.4 Cell biology methods

2.2.4.1 Cryopreservation of mammalian cell lines ___ 37

2.2.4.2 Cell culturing and maintenance ___ 37

2.2.4.3 Stress model for stress induction___ 37

2.2.4.4 Immunocytochemistry __ 38

2.2.4.5 Subcellular fractionation__ 38

2.2.4.6 Cell lysis and protein extraction___ 39

2.2.4.7 Tau transfections _ 39

2.2.4.8 SWATH-MS for global proteomics___ 40

2.2.5 Biochemical methods ___ 42

2.2.5.1 MTS assay ___ 42

2.2.5.2 Trypan blue exclusion assay ___ 42

2.2.6 Animal time course and sample collection $\_42$

2.2.7 Statistical analysis __ 43

3 Results__ 45

3.1 RNA pull-down assay and mass spectrometry analysis of RNA-binding proteome__ 46

3.1.1 Global enrichment profile of RNA-binding proteome

3.1.2 Functional categorization of RBP candidates _ 52

3.1.3 Classification of known and putative/novel RBP candidates _ 55

3.1.4 Prion-like domain (PLD) prediction

3.1.5 Target candidates from proteomic study _ 58 
3.2 Pathological characterization of target RBP (SFPQ) in the postmortem human brain 59

3.2.1 SFPQ is dysregulated in $r P A D$ and $S C J D$ brains 59

3.2.2 SFPQ mislocalization and co-localization with SG marker TIA-1 in the rPAD brain 63

3.2.3 SFPQ is co-localized with phospho-tau in neurofibrillary tangles in the rPAD brain 66

3.2.4 Tau oligomers are co-localized with SFPQ in the rpAD brain 70

3.3 Translational study of SFPQ in cellular models 72

3.3.1 Role of SFPQ towards stress axis 72

3.3.1.1 Characterization of TIA-1-positive SGs 72

3.3.1.2 Tau phosphorylation is increased after stress induction 74

3.3.1.3 Tau and phospho-tau are recruited into SGs 75

3.3.1.4 Endogenous SFPQ redistributes into the cytoplasm and assembles with SGs upon oxidative stress treatment 77

3.3.1.5 SFPQ co-localizes with tau and phospho-tau in cytoplasmic granules 80

3.3.1.6 Biochemical characterization of stress granule components 81

3.3.2 Role of SFPQ in the tau axis 84

3.3.2.1 SFPQ downregulation induced by human tau expression 84

3.3.2.2 Proteomic changes associated with SFPQ downregulation after human tauexpression 86

3.3.2.2.1 Canonical pathway analysis 89

3.3.2.2.2 Disease- and function-based protein networks 90

3.3.2.2.3 Protein network associated with RNA damage and repair, protein synthesis, cancer 90

3.3.2.2.4 Protein network associated to cell morphology, cellular assembly and organization, DNA replication, recombination, and repair 91

3.4 Translation of SFPQ-tau-TIA-1 in the 3xTg-AD mice model 92

3.4.1 Differential expression of tau in AD mice 93

3.4.2 Dysregulation of SFPQ at early and late-symptomatic disease stages 93

3.4.3 Alterations in TIA-1 levels at early pre-symptomatic and terminal stage of the disease 94 


\section{Contents}

4.1.1 Functional analysis of MS results 97

4.1.1.1 Proteins involved in RNA metabolism and stress response 98

4.1.2 Canonical and putative RNA-binding candidates 99

4.1.3 Prion-like-domain (PLD)-containing proteins 99

4.2 Pathological characterization of SFPQ in the human brain 100

4.2.1 SFPQ dysregulation in the rPAD and SCJD brains 100

4.2.2 SFPQ dislocation in the brain of rpAD patients 101

4.2.3 SFPQ co-localization with the SG marker TIA-1 in the rPAD brain 102

4.2.4 SFPQ and neurofibrillary tangles 103

4.2.5 SFPQ co-localizes with oligomeric tau in the rpAD 104

4.2.6 Differential regulation of SG marker TIA-1 in disease-subtype-specific manner 106

4.3 Translational aspects of SFPQ in cellular models 107

4.3.1 Cellular model of stress 107

4.3.2 SFPQ recruitment into SGs after oxidative stress treatment 107

4.3.3 Tau, TIA-1 and SFPQ in stress granules 108

4.3.4 Biochemical characterization of stress-granule components 110

4.3.5 Role of SFPQ towards tau axis 111

4.3.5.1 Dysregulated pathways associated with tau-mediated downregulation of SFPQ

4.4 Dysregulation of SFPQ, tau, and TIA-1 in 3xTg-AD mice 114

4.5 Conclusion 116

5 Annexure

5.1 RNA-binding protein candidates from mass spectrometry analysis. 118

6 Bibliography 142

Acknowledgements 173

Biography 174 


\section{Abbreviations}

ACN

AD

ALS

APP

$A \beta$

$\mathrm{BH}$

CA1

CERAD

Cont.

CDNA

$\mathrm{dd}_{2} \mathrm{O}$

DAPI

DIA

DMSO

DEPs

DNA

DTT

EC

EDTA

EOAD

ESI

FA

FAD

FBS

FC

FDR

FTD

GAPDH

GSK3- $\beta$

GO

HD

HMW

$\mathrm{hr}$

IAA

IB

IF

$\lg G$

$\mathrm{IHC}$

I $\beta-1$

$\mathrm{kDa}$

LFQ-MS
Acetonitrile

Alzheimer's disease

Amyotrophic lateral sclerosis

Amyloid-precursor protein

Amyloid- $\beta$

Benjamini-Hochberg correction

Cornu ammonis 1

Consortium to Establish a Registry for Alzheimer's Disease

Control

Complementary DNA

Double distilled water

4',6-Diamidino-2-phenylindole (double

stranded DNA staining)

Data independent acquisition

Dimethyl sulfoxide

Differentially expressed proteins

Deoxyribonucleic acid

Dithiothreitol

Entorhinal cortex

Ethylenediaminetetraacetic acid

Early onset $A D$

Electrospray ionization

Formic acid

Familial Alzheimer's disease

Fetal bovine serum

Fold change

False discovery rate

Fronto-temporal dementia

Glyceraldehyde 3-phosphate dehydrogenase

Glycogen synthase kinase 3- $\beta$

Gene Ontology

Huntington's disease

High molecular weight

Hour

lodoacetamide

Immunoblotting

Immunofluorescence

Immunoglobulin G

Immunohistochemistry

Importin- $\beta-1$

Kilodalton

Label-free quantification-mass spectrometry 
Lauria-Bertani

Low molecular weight

Late onset Alzheimer's disease

Microtubule-associated protein tau

Mild cognitive impairment

Mini-Mental State Examination

Mass spectrometry

Neurofibrillary tangles

Nonidet P-40

Phosphate-buffered saline

Phosphate-buffered saline-Tween-20

Penicillin-Streptomycin

Parkinson's disease

Prion-like amino acid composition

Prion-like domain

Prion protein gene

Presenilin 1

Polyvinylidene difluoride

Phosphorylated tau

Quantitative real time-polymerase chain reaction

Quadrupole-time-of-flight

RNA-binding domain

RNA-binding protein

Ribonucleic acid

$[\mathrm{PIN}+]$ prion protein

Ribonucleoprotein

Reactive oxygen species

Pearson's linear correlation coefficient

Rapidly progressive Alzheimer's disease

Revolutions per minute

Room temperature

Sporadic Alzheimer's disease

Sporadic Creutzfeldt-Jakob disease

Sodium dodecyl sulfate

Sodium dodecyl sulfate-polyacrylamide gel electrophoresis

Sporadic Alzheimer's disease

Stress granule

Splicing factor proline and glutamine rich

Sequential window acquisition of all theoret-

ical mass spectra-Mass spectrometry

Eukaryotic peptide chain release factor

GTP-binding subunit

Tubulin-associated unit

Tris-buffered saline 
TBS-T

TEMED

TFA

TIA-1

tM

Tris

UTRs

VCP
Tris-buffered saline-Tween-20

Tetramethylethylenediamine

Triflouroacetic acid

T-cell intracellular antigen-1

Threshold Mander's coefficient

Tris(hydroxymethyl)aminomethane

Untranslated regions

Valosin-containing protein 


\section{Summary}

Alzheimer's disease (AD) is the most prevalent cause of dementia. Typically, $A D$ is characterized as a slow progressive dementia with an average disease duration of eight years. Classically, $A D$ is categorized into two subtypes: the first subtype includes cases with spontaneous onset, termed sporadic AD (spAD), while the second subtype (familial $A D$ ) includes cases exhibiting mutations in genes encoding presenilin-1, presenilin-2, and amyloid-precursor protein (APP). Recently, a rapidly progressive variant of Alzheimer's disease (rpAD) was identified, in which patients exhibit a rapid cognitive decline and/or short disease duration (average of 4 years). It is known that SPAD and rpAD share core neuropathological features, but unfortunately the altered molecular processes, which eventually lead to these variable rates of progression, remain elusive. To this end, we aimed to explore the emerging role of RNA-binding proteins (RBPs) in these two AD subtypes and in sporadic CreutzfeldtJakob disease (sCJD), another rapidly progressive form of dementia.

In the current study, we utilized an RNA pull-down approach from brain samples followed by mass spectrometry analysis to comprehensively interrogate RNA-binding protein (RBP) complexes; these were examined in human brain frontal cortex samples from three groups of patients, namely spAD, rpAD, and SCJD, as well as controls. Using a combination of bioinformatic and computational techniques, significant targets from the proteomic study were identified and prioritized for further characterization. The first set of analyses investigated differential expression of a target RBP termed splicing factor proline and glutamine rich (SFPQ) at both the protein and mRNA level, its accumulation as well as its possible interactions with tau protein and stress granules (SGs) in the postmortem brains. To investigate a mechanistic link between SFPQ and the pathogenesis and progression of AD, it was furthermore studied in two cellular models - the cellular model of stress and the tau-pathology model - given that in the human brain associations exist between SFPQ, stress granules and tau protein. Finally, SFPQ and associated proteomic signatures were studied at the pre-symptomatic and symptomatic stages of the disease in the $3 \times \mathrm{Tg}$ $A D$ mice model, in order to uncover very early changes occurring during the disease progression. 
In the present study, the RNA-binding proteome from Alzheimer's and SCJD subtypes were identified and characterized. The proteomic investigation, in combination with several bioinformatic and computational approaches, highlighted quantitative and qualitative changes in the identified RNA-binding proteome in a diseasesubtype-specific manner. We identified a dysregulation pattern both at the protein and mRNA level, including the dislocation of the RNA-binding protein SFPQ as a novel pathological target in the rapidly progressive subtype of AD. The SFPQ protein is involved in multiple functions in the brain, including splicing, transcription, and transport of mRNAs. This suggests that the dysregulation/dislocation of SFPQ lead to defects in these functions, which aggravate the neurodegenerative processes and eventually contribute to the rapid progression.

Furthermore, co-immunofluorescence analysis revealed a change in the fluorescence pattern of phosphorylated tau ( $p$-tau) along with SFPQ, with a complete nuclear depletion of both proteins and co-localization in the perinuclear/cytoplasmic area. This indicated that there are changes in the function of both nuclear tau and SFPQ. The cytoplasmic SFPQ showed co-localization with TIA-1, a marker of stress granules (SGs). In parallel with human brain findings, our study of the cellular model of stress indicated that SFPQ and tau translocate into the cytoplasm to form SGs after oxidative stress treatment. This translocation of the two proteins into the SGs provides a possible mechanism for the observed depletion/dislocation of SFPQ and nuclear tau in postmortem human brains from rpAD cases. At initial phases of the disease, kinases phosphorylate not only tau but also SFPQ, leading to their translocation into perinuclear/cytoplasmic area and their incorporation into stress granules. Chronic stress, such as that which occurs during the disease, may convert these physiological stress granules into pathological stress granules, which can lead to the abnormal sequestration of SFPQ and nuclear tau in the cytoplasm resulting in an overall depletion from the nucleus. Of note, co-localization of SFPQ with oligomeric tau indicates a potential role of SFPQ in oligomerization and misfolding of the tau protein, which appears as a major hallmark of $A D$.

The significant reduction in SFPQ levels observed after human tau expression (taupathology model) in vitro and in the postmortem brains of rpAD subjects, suggests a causal role of tau in the downregulation of SFPQ. Quantitative proteomic analysis 
using Sequential Window Acquisition of all THeoretical fragment ion spectra-MS (SWATH-MS) in combination with functional characterisation illustrated two major themes (global translation reduction and DNA repair) that were altered as a consequence of the combinatorial effect of tau toxicity and SFPQ downregulation in this tau-pathology model.

Finally, the transgenic 3xTg-AD mice model uncovered specifically pre-symptomatic changes of target proteomic signatures. The levels of SFPQ and TIA-1 were already significantly elevated at an early pre-symptomatic phase of the disease in 3xTg-AD mice, suggesting that these proteins could be of potential significance as early therapeutic targets. This upregulation of the two SG components SFPQ and TIA-1 indicates active functions of the SG machinery at the early pre-symptomatic stage of the disease implicating pre-tangle stress, which coincides well with the observed acute phase oxidative stress-mediated upregulation of phospho-tau, TIA-1, and SFPQ in our cellular model of stress. Furthermore, the parallel reduction of SFPQ, which was found in the late symptomatic stage in 3xTg-AD mice and in the postmortem brains of patients with rapidly progressive forms of dementias (rpAD and prion disease), suggests that SFPQ may function as a common marker associated with rapid progression of these diseases.

On the basis of the findings from the current study, it can be concluded that the dislocation and dysregulation of SFPQ and nuclear tau, the subsequent DNA-related anomalies and aberrant dynamics of SGs in association with pathological tau represents a novel pathway which contributes to rapid progression in AD. Early presymptomatic changes in SFPQ indicate its relevance as an early therapeutic target. Reestablishing nuclear localization/expression of SFPQ might be a promising strategy to rescue neurodegeneration or to slow down the progression of the disease. 


\section{Introduction}

\subsection{RNA-binding proteins (RBPs)}

The post-transcriptional regulatory mechanisms of neuronal gene expression are fast and effective processes that fine-tune the proteome of a cell in the brain to an everchanging microenvironment (Glisovic et al., 2008; Janga and Mittal, 2011; Richter and Klann, 2009; Wang and Szaro, 2016). These gene regulatory mechanisms are controlled by a group of special proteins known as RNA-binding proteins (RBPs) (Glisovic et al., 2008; McMahon et al., 2016). RNA-binding proteins are key regulators in RNA processing and translational control as they are complementary for RNAs, regulating all aspects of RNA metabolism; this includes alternative splicing, packaging, transport, stabilisation, translation, degradation, and the facilitation of RNA-interactions with other macromolecules (Fig. 1) (Anderson and Kedersha, 2009; Marchese et al., 2016). More than 1500 RBPs have been described in the human cell (Castello et al., 2012; Gerstberger et al., 2014; Hentze et al., 2018). RNA-binding proteins interact with cis-regulatory elements in the mRNA to form ribonucleoprotein (RNP) complexes, also known as granules (Wahl et al., 2009), thereby controlling the function/expression of their target RNAs (Fig. 1) (Zhou et al., 2014).

All RNP granules are composed of RBPs associated with mRNAs in their untranslated regions (5-' or 3-'UTR) or in the coding regions (Anderson and Kedersha, 2009; Martin and Ephrussi, 2009). Transport granules are responsible for localization and storage of mRNAs for localized protein synthesis (Ramaswami et al., 2013; Xing and Bassell, 2013). Stress granules (SGs) are reversible membrane-less aggregates in the cytoplasm which are formed in response to different environmental stresses (e.g. heat shock, oxidative stress, nutrient deprivation etc.); they are then resolved after the removal of stress (Anderson et al., 2015; Guo and Shorter, 2015; Jain et al., 2016). These granules have a pivotal role in stress response, sequestering mRNAs to sort them for their storage or decay (Jain et. al., 2016). Degradation of mRNA occurs in processing bodies that are made up of proteins involved in degradation, surveillance of mRNA, repression of translation, and RNA-dependent silencing processes (Fig. 1) (Guo and Shorter, 2015; Jain and Parker, 2013; Marchese et al., 2016). 


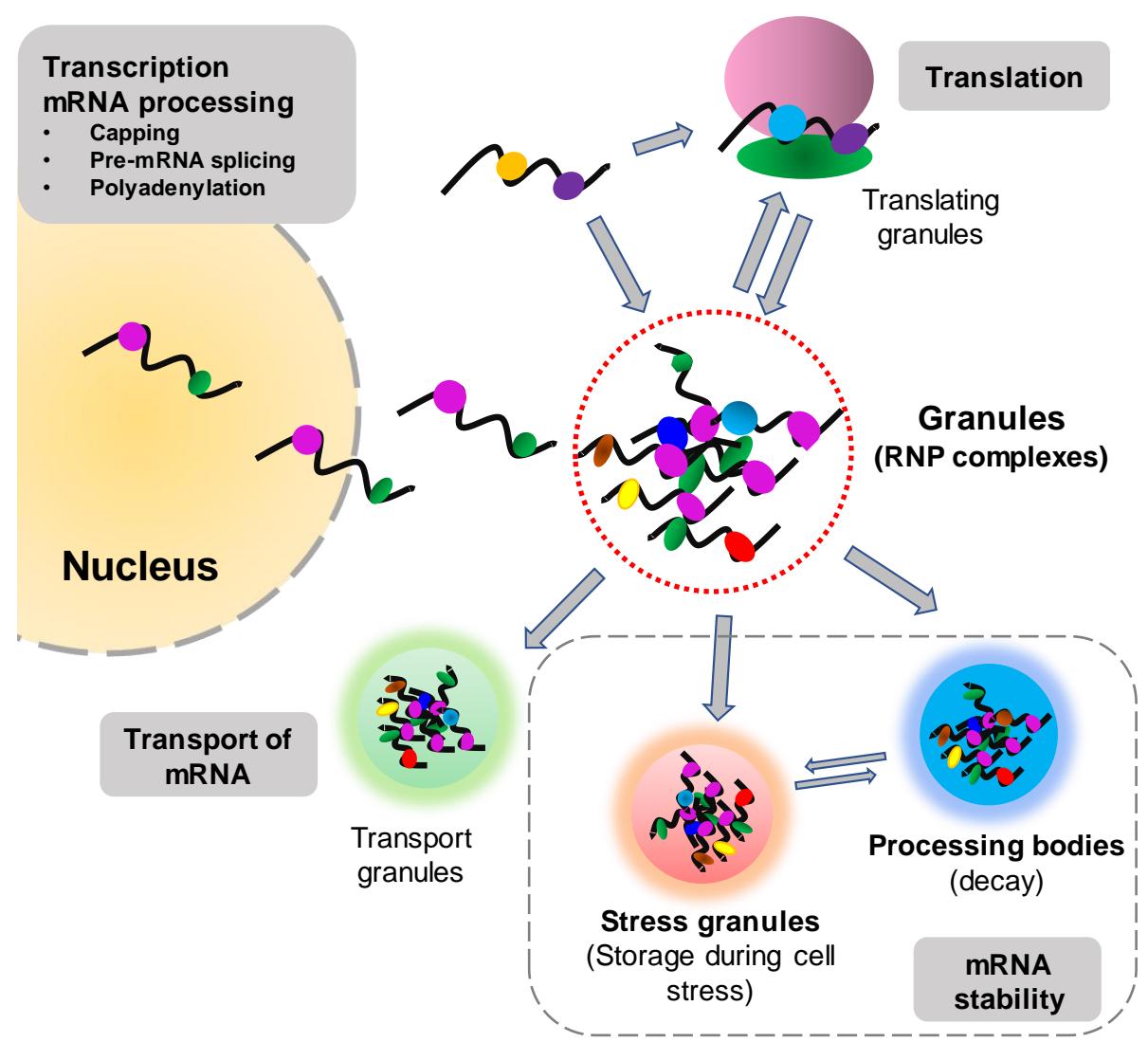

Figure 1: Functions of RNA-binding proteins through RNA-granule assembly. This figure highlights different steps of the RNA life cycle which are controlled by RBPs (grey rectangles). In the nucleus, RBPs are responsible for regulation of transcription, pre-mRNA processing, and export of newly synthesized mRNAs from the nucleus. Several types of granules present in the cytoplasm with their functions are described here. RNA granules are composed of an array of different proteins including RBPs, ribosomal subunits, helicases, translation factors and decay enzymes (Anderson and Kedersha, 2006). Disordered domains of RBPs contribute to dynamic remodeling of these granules by creating landing platform for RNA-protein and protein-protein interactions. Translating granules are responsible for translational control of the RNA. Stress granules safeguard mRNA and store them during stress response. Processing bodies are responsible for mRNA decay. Both stress granules and processing bodies are dynamic structures and share their components. Transport granules mediate the translocation of mRNAs through the long axons in neurons for de novo protein synthesis at the synapse (adapted and modified from Coppin et al., 2018).

The interaction between RBPs and RNA is achieved by special domains in RBPs, known as RNA-binding domains (RBDs). RNA-binding proteins bind with RNA either by recognizing specific sequence elements in the target transcript or by recognizing secondary structural features in the RNA molecule (Castello et al., 2016; Wright and Dyson, 2015). Therefore, binding specificity is dependent on both the secondary structural features of the RNA and the bound RBPs (Ding et al., 2014; Gosai et al., 2015; Li et al., 2012). Typically, binding sites for RBPs are present in the untranslated regions of RNA but some sites can also be present in the coding regions as well 
(Dassi et al., 2012; Pickering and Willis, 2005). Sequence elements in the $5^{\prime}$-UTRs control the expression pattern of proteins, e.g. ribose-methylation of the cap, 5terminal polypyrimidine sequences, and secondary structural elements. Sequences in the $3^{\prime}$-UTR region are responsible for regulation of stability, translational control, and localization (Lunde et al., 2007; Wurth, 2012).

RNA recognition is complicated by the flexibility in the structure of the RNA. A singlestranded RBP might have strong sequence preferences, but the accessibility of an individual strand can depend on surrounding RNA structures (Duss et al., 2014; Helder et al., 2016). Furthermore, posttranslational modifications of RBPs are crucial, not only in diversifying their RNA-binding specificities but also in intracellular localization and metabolic functions (Glisovic et al., 2008). Dysfunctional RBPs are emerging as key players in many neurological diseases (Maziuk et al., 2018).

According to classical assumptions, RNA-protein interactions are dependent on welldefined, ordered globular domains. These well-structured RNA-binding domains are categorized into four main families: the zinc-finger domain (Brown, 2005), the Khomology domain (Valverde et al., 2008), the RNA recognition motif (Clery et al., 2008), and the double-stranded RNA-binding domain (Banerjee and Barraud, 2014). Until recently, RNA-protein interactions were assumed to be mediated mainly by these classical domains; however, new research, which characterizes other types of RNA-binding domains, has added more complexity to the intricate mesh of RNAprotein complexes (Balcerak et al., 2019).

The binding of RBPs with RNA is not only specific but can also be non-specific, through auxiliary domains which greatly increase their functional diversity. Auxiliary domains consist of intrinsically disordered regions, which are composed of repetitive sequences of characteristic amino acids and a low percentage of hydrophobic amino acids. These domains enable the RBPs to form dynamic disordered structures ranging from collapsed globules to extended coils (Dyson and Wright, 2005; Varadi et al., 2015; Wright and Dyson, 2015). Intrinsically unstructured regions in the RNA-binding proteins are important in two ways. Firstly, these segments establish extended yet conserved electrostatic boundaries with RNAs via induced fit. Secondly, flexibility in their conformation enables them to bind different RNA targets, providing multifunctionality while also ensuring specificity (Varadi et al., 2015; Calabretta and 
Richard, 2015). These regions are responsible for reversible phase transition, leading to formation of liquid droplets, hydrogels, and aggregates or fibrils (Brangwynne, 2013; Hyman et al., 2014). RNA-protein interactions mediated by auxiliary domains affect many aspects of RNA-processing; their disruption, therefore, can potentially cause protein disorders (Varadi et al., 2015; Calabretta and Richard, 2015).

An important subset of low-complexity domains are prion-like domains (PLDs), mainly comprised of uncharged polar residues and glycines, showing similarities with the prion protein of yeast (Couthouis et al., 2011; Lancaster et al., 2014). These PLDs enable several proteins of yeast, e.g. Sup35 and Rnq1, to form infectious structures, termed prions (Alberti et al., 2009; King et al., 2012; Toombs et al., 2010; Wickner et al., 2015). Deletion of these prion domains precludes access to the prion state (Masison et al., 1997), and the addition of this region to otherwise innocuous proteins is sufficient to induce prion-like behaviour (Li and Lindquist, 2000; Tyedmers et al., 2010).

The development of bioinformatics algorithms has led to the identification of bonafide prion domains (Alberti et al., 2009; Couthouis et al., 2011; King et al., 2012; Toombs et al., 2010). These algorithms scan amino acid compositions to screen the human genome for proteins with PLDs. One of the updated PLD detection algorithm is PLAAC (Prion-Like Amino Acid Composition) (Lancaster et al., 2014), which has been used for PLD prediction for several organisms.

There are about 240 human proteins with PLDs that have been identified by PLAAC. Of these, 70 are RBPs, suggesting a beneficial and essential role of these domains. Prion-like domains are essential for RBP functions and enable them to undergo liquid-liquid phase separation (LLPS). This phase separation is the basis for the formation of higher-order structures, including oligomers and several membrane-less granules (Fig. 2) (Toretsky and Wright 2014; Verdile et al., 2019). However, this LLPS property renders prion-like-domain-containing proteins prone to misfold and aggregate via aberrant phase transitions (Fig.2) (Harrison and Shorter, 2017; Verdile et al., 2019). Prion-like domains form mesh-like networks in vitro, manifesting as hydrogels (Kato et al., 2012). These hydrogels are different from amyloid material and signify a functional amyloid (Hennig et al., 2015). Due to this special property of functional aggregation, PLD-containing proteins have gained attention in recent years in 
protein aggregation disorders, e.g. amyotrophic lateral sclerosis (ALS), Alzheimer's, and prion diseases (Harrison and Shorter, 2017; March et al., 2016; Wolozin, 2012).

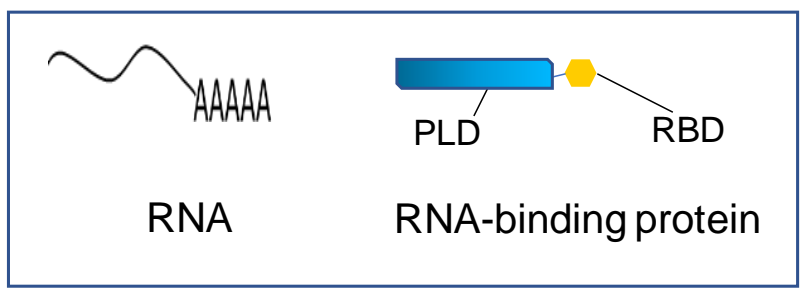

Polymeric, amyloid
like fiber state (RNP granules)

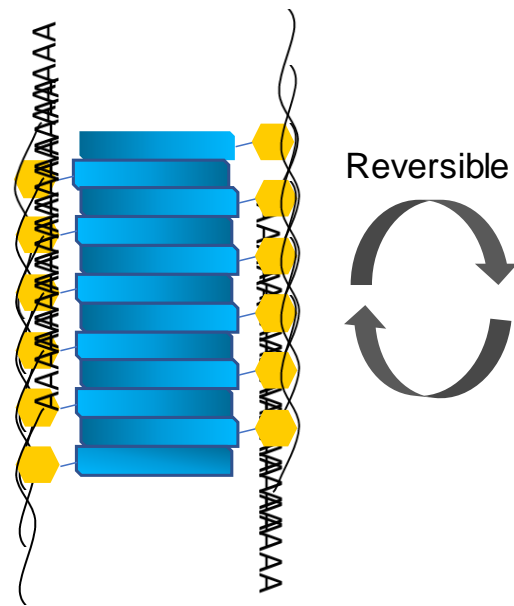

Monomeric/dimeric soluble state
Pathogenic aggregated state

Figure 2: Prion-like granule assembly by RNA-binding proteins with prion-like domains. The prion-like domains enable RBPs to exist in one of three states: a soluble state (monomeric), an amyloid-like fiber state (polymeric) or a pathogenic (aggregate) state. This polymeric amyloid state is the basis of formation of different types of granules, e.g. stress granules, transport granules, and processing bodies. The conversion between the first two states is reversible, which means RBPs can both enter and exit a prion-like state. Transition to $3^{\text {rd }}$ state is pathogenic and is irreversible (Kato et al., 2012) (adapted from Gao and Arkov, 2013).

Regulation of RNA-biology is highly complex, due to the heightened demands of RBP functions in the neurons. RNA-binding proteins are important for neurons in two distinct ways. Firstly, alternative splicing is particularly active in the neurons, as compared with other tissues (Li et al., 2007; Yeo et al., 2004). This posttranscriptional regulatory mechanism (alternative splicing) is dependent on RBPs. Secondly, it is RBPs that ensure safe transportation of mRNAs from the nucleus to the cytoplasm, dendrites and long axons, thus preventing their premature degradation and translation during their journey (Fig. 3) (Anji and Kumari, 2016; Holt and Bullock, 2009; Zhang and Poo, 2002; Zhou et al., 2018). In addition, RNA-binding proteins are efficient and fast regulatory hubs, helping the neuron to manage the strains of an ever- 
changing microenvironment, including synaptic depolarization responses, depression, oxidative stress, misfolded proteins, reduced nutrient availability, and apoptosis (Sephton and Yu, 2015; Zhou et al., 2018).

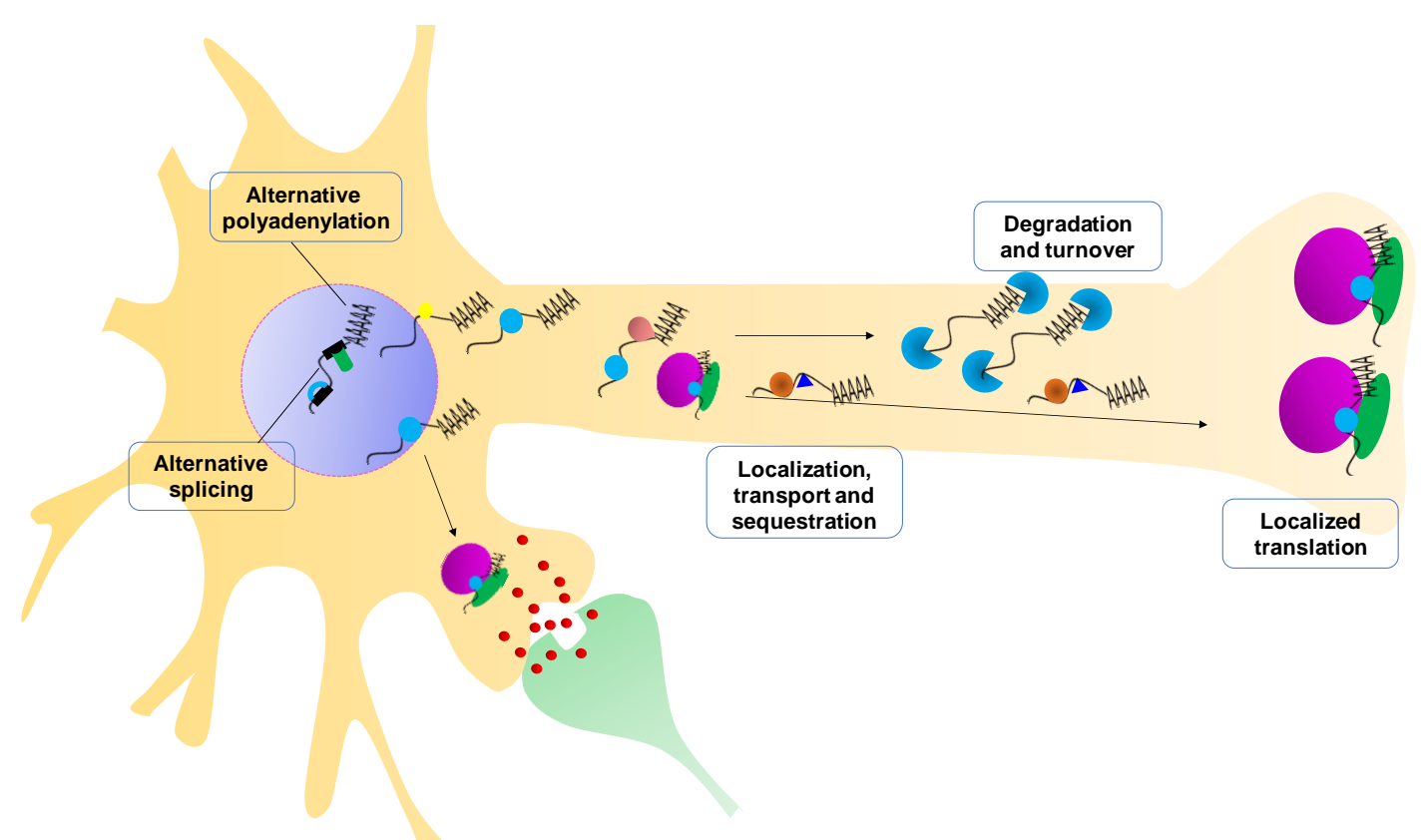

Figure 3: RNA-binding proteins in the neuron. RNA-binding proteins are very important in the neurons in order to fulfill heightened demand of dynamic RNA-RBP processes in the neurons. Firstly, alternative splicing is particularly active in the neurons compared with other tissues. Secondly, neurons need RBPs to transport mRNAs through long axons to distal parts of the neurons, e.g. the synapse for de novo protein synthesis. As a result, dysfunction of the RNA-binding proteins leads to defects in post-transcriptional gene regulatory mechanisms, resulting in neurodegenerative disorders (adapted and modified from Zhou et al., 2018).

In several neurodegenerative diseases, alterations in the dosage and dynamics of RBPs, including expressional changes, mutations, aberrant interactions or altered RNA-processing, are emerging as a major pathological feature (Castello et al., 2013; Liu et al., 2017; Maziuk et al., 2018; Nussbacher et al., 2015). Dysregulation of many RBPs, including FUS, TDP-43, hnRNPA1 and ATXN2, have been demonstrated in amyotrophic lateral sclerosis, frontotemporal lobar degeneration and/or spinocerebellar ataxia (Table 1) (Maziuk et al., 2017). Other RBPs have also been shown to co-aggregate with aggregated-prone protein inclusions in AD, Huntington's disease (HD) and Creutzfeldt-Jakob disease (CJD) (Table 1) (Goggin et. al., 2008; Maziuk et al., 2017; Nussbacher et al., 2015; Zhou et al., 2014). 
Table 1: Different RBPs associated with neurological diseases.

\begin{tabular}{ll}
\hline RNA-binding proteins & Associated diseases \\
\hline TAR DNA-binding protein 43 (TDP-43) & ALS, FTLD, AD, HD \\
T-cell intracellular antigen 1 (TIA-1) & ALS, FTLD, AD \\
Ras GTPase-activating protein-binding protein 1 & \\
(G3BP1) & ALS, FTLD, AD \\
Tristetraprolin (TTP) & ALS, FTLD, AD \\
Fused in Sarcoma (FUS) & ALS, FTLD \\
Ewing Sarcoma protein (EWS) & ALS, FTLD \\
TATA-Box Binding Protein Associated Factor 15 & \\
(TAF15) & ALS, FTLD \\
Heterogenous Ribonucleoprotein Particle A1/A2 & ALS, FTLD \\
(hnRPA1/A2) & ALS, PD \\
Angiogenin (ANG) & ALS, SMA \\
Survival of motor neuron (SMN1) & ALS \\
Matrin-3 (MATR3) & ALS \\
Ataxin-2 (ATXN2) & ALS \\
Optineuin (OPTN) & FXS \\
Fragile X mental retardation protein (FMRP) & \\
\hline ALS: Amy &
\end{tabular}

ALS: Amyotrophic lateral sclerosis, FTLD: Frontotemporal lobar degeneration, AD: Alzheimer's disease, HD: Huntington's disease, PD: Parkinson's disease, FXS: Fragile X syndrome, SMA: Spinal muscular atrophy (Maziuk et al., 2017).

One by one, connections are being discovered between RNA-binding proteins with prion-like domains and neurodegenerative disorders (Li et al., 2013; March et al., 2016). Recently, this paradigm of RNA-binding proteins has been extended to $A D$, where pathological aggregates of TIA-1 (TIA-1: cytotoxic granule-associated RBP), an RNA-binding protein with prion-like domain, have been linked to tau neurofibrillary tangles (NFTs) in the brain (Vanderweyde et al., 2012, Vanderweyde et al.,2016). Furthermore, co-aggregation of components of the spliceosomal complex with the tau protein has been reported in both sporadic and familial AD cases, but not in other tauopathies (Bai et al., 2013; Bishof et al., 2018; Diner et al., 2014; Sengupta et al., 2018).

\subsection{Alzheimer's disease}

Alzheimer's disease is the most prevalent cause of dementia with progressive neurodegeneration, affecting 40-50 million people around the globe (GBD Dementia Collaborators, 2019; Prince et al., 2013). This number is predicted to increase threefold by 2050 (Prince et al., 2013; Prince et al., 2015). The prevalence of AD is quite high in North America and Europe, compared with less developed countries, though a sharp increase has been observed in India, China and Latin America in recent 
years (Ferri et al., 2005; Kalaria et al., 2008). In America alone, the financial burden of maintaining AD patient's health care amounts to $\sim \$ 203$ billion annually. Due to a total lack of therapeutic interventions for the treatment and prevention of $A D$, the costs are expected to reach $\$ 1.1$ trillion annually by the year 2050 (Alzheimer's Association, 2019).

Alzheimer's disease has two sub-classifications: early-onset (EOAD) or familial $A D$ (FAD), or late-onset (LOAD) or sporadic $A D(S A D)$. For FAD, symptoms usually appear earlier than SAD, typically ranging between 30-50 years of the age (Bertram et al., 2010; Goate and Hardy, 2012; Sanabria-Castro et al., 2017). Autosomal dominant mutations in amyloid-precursor protein (APP), presenilin-1, and -2 genes have been shown to cause familial AD (Blennow et al., 2006). Known genetic causes of $A D$ only account for a small percentage of cases (less than 1\%). Alzheimer's disease cases, which are not associated with any genetic mutations, are known as sporadic AD (Mendez, 2017).

Clinically, $A D$ is characterized by continuous memory deficits and dysfunction of other cognitive abilities. At initial phases, the major symptoms are centered on episodic memory. With progression of the disease, topographical difficulties emerge, alongside problems with multi-tasking and loss of confidence. By the time a patient is diagnosed with $A D$ dementia, symptoms have typically become more sever, interfering with activities of daily life (Scheltens et al., 2016). At later stages of the disease, other deficits may also emerge, including impaired mobility, behavioural abnormalities, hallucinations and delusions. Severe stages of the disease are accompanied by a complete loss of various cognitive functions, impaired motor functions (e.g. chewing and swallowing) and linguistic problems. Most of the patients are bedridden at this stage, and die of inanition or secondary illnesses, e.g. infections and ulcers (Förstl and Kurz, 1999; Tarawneh and Holtzman, 2012).

Neuropathologically, AD is characterized by two cardinal hallmarks: intracellular tangles of misfolded tau protein in conjunction with extracellular plaques of aggregated amyloid- $\beta$ (A $\beta$ ) peptide (Cushman et al., 2010; Perl, 2010), together with neuronal and synapse loss (Nelson et al., 2009; Selkoe and Hardy, 2016; Perl, 2010). The A $\beta$ plaques originate from the aggregation of $A \beta$ peptides (40-42 amino acid long), produced by sequential cleavage of APP by the $\beta$ - and $\gamma$-secretases. An imbalance be- 
tween the production and clear mechanisms for $A \beta$ peptides leads to precipitation of A $\beta$ pathology (Selkoe and Hardy, 2016).

The amyloid hypothesis posits $A \beta$ pathology as the primary pathological feature of the disease (Hardy and Higgins, 1992), triggering a cascade of further pathological events, including the formation of neurofibrillary tangles of hyperphosphorylated tau, neuroinflammation, oxidative stress, and neuronal loss (Hardy and Higgins, 1992; Reitz, 2012; Tanzi and Bertram, 2005). Unfortunately, therapeutic interventions targeting $A \beta$ have failed to improve cognitive functions in $A D$ (Doody et al., 2014; Lovestone et al., 2015). One possible reason for the failure of these therapeutic strategies is an incomplete understanding of the mechanisms leading to neurodegeneration in AD. Mounting evidence has shifted the focus towards tau as a more promising therapeutic target for AD (Cao et al., 2018). The discovery of pathogenic mutations in the tau gene in familial cases of frontotemporal dementia (FTLD-17: FTLD with parkinsonism linked to chromosome 17) has demonstrated a clear link between the dysfunction of tau and neurodegeneration (Goedert et al., 2000).

Tau, suggested by some to be the "holy grail of dementia," is a protein initially described as a dull executor of pathological effects associated with amyloid $\beta$. In AD, tau is hyperphosphorylated, misfolded, oligomerized, aggregated, and mislocalized (Grundke-lqbal, 1986; Ren and Sahara, 2013; Vanderweyde et al., 2016). Since the initial discovery of tau in 1975, the field has focused on its role in microtubule stabilization by binding with polymerized tubulin in the axons (Weingarten et al., 1975). Over the last few decades, several studies have reported multiple functions and localizations of tau protein. Specifically, its localization in the nucleus (both phosphorylated and non-phosphorylated) (Bukar et al., 2016) and cytoplasm under conditions of oxidative stress has been demonstrated (Vanderweyde et al., 2016). Unfortunately, the significance of multiple localization types is not yet clear.

Recent evidence suggests a novel pathological feature of tau in relation to cytoplasmic stress granules, through which tau disrupts cellular homeostasis. RNA-binding proteins, like TIA-1, co-localize with hyperphosphorylated tau and aggravate tau pathology (Fig. 4) (Vanderweyde et al., 2012; Vanderweyde et al., 2016). This interaction between tau and TIA-1 in stress granules has been shown to enhance taumediated neurodegeneration in primary hippocampal cultures, which can be rescued 
by reduction of TIA-1 protein (Fig. 4) (Vanderweyde et al., 2016). Likewise, extracelIular tau, after internalization and hyperphosphorylation, has been shown to alter SG dynamics, supporting the notion that secreted tau has a role in the formation of pathological SGs (Brunello et al., 2016). All these evidences link tau pathology to dysfunctional RBPs and pathological stress granules, highlighting an important role of RBPs in AD.

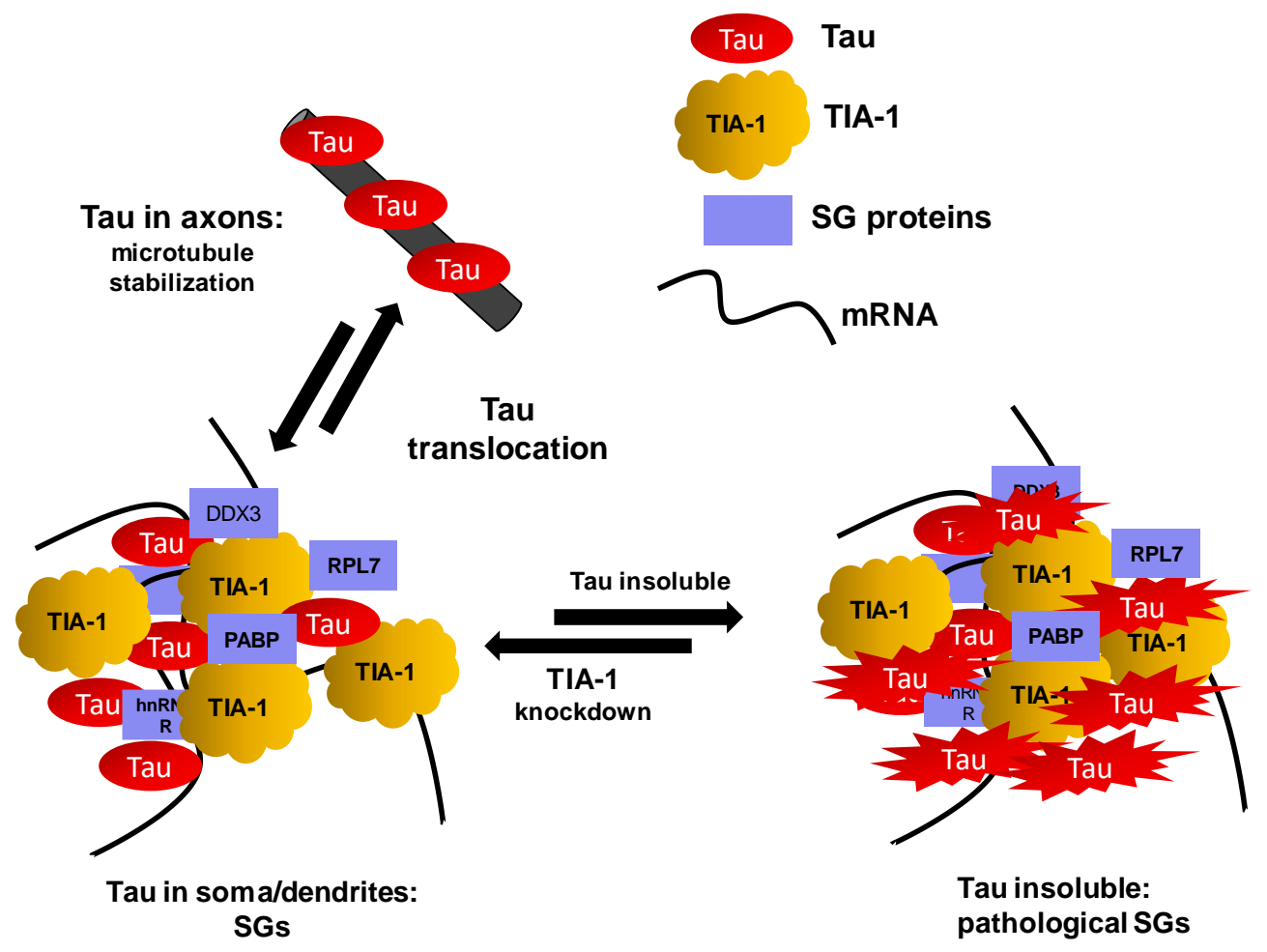

Figure 4: The interplay between tau and RNA-binding proteins in the SGs: Tau is normally present in the axons, but stress induces translocation to somatodendritic compartments. RNA-binding proteins, e.g. TIA-1 with both nuclear and cytoplasmic functions, keep on translocating between cytoplasmic and nuclear regions. Although TIA-1 is predominantly a nuclear protein, stress leads to rapid shuttling into the cytoplasm, where its interaction with tau promotes SG formation. This association also enhances tau misfolding and aggregation and increases the size of SGs by influencing the RNA-binding protein composition of SGs. Furthermore, this contact increases the tendency of tau to form sarkosyl-insoluble aggregates and stabilize SGs. These deleterious effects can be rescued by TIA-1 reduction in cultured neurons (adapted and modified from Vanderweyde et. al., 2016).

The current neuropathological assessment of $A D$ is based on updated criteria released by the National institute of Aging (NIA). Classification of AD neuropathologic changes is achieved according to three different staging themes: Thal stages for distribution of amyloid- $\beta$ deposits (Thal et al., 2002), neurofibrillary tangle pathology with Braak stages (Braak and Braak, 1991; Braak et al., 2006), and the occurrence and severity of neuritic plaques according to the Consortium to Establish a Registry for Alzheimer's Disease (CERAD) (Fillenbaum et al., 2008; Mirra et al., 1991). Com- 
bining these three standards (Amyloid: $A$, Braak: B, CERAD: C) to the $A B C$ method provides an estimate of a no, low, medium or high pathology (Montine et al., 2012).

The genetic and non-genetic risk factors associated with $A D$ are significant, because they give clues into the predispositions of the disease process prior to onset. Furthermore, they provide basis for classification of individuals with increased risk for the disease. Diverse non-genetic risk factors have been linked with $A D$ including cardiovascular diseases, hypertension, type 2 diabetes, obesity, traumatic injury to the head, life style (poor diet, physical inactivity, smoking etc.), and depression (Crous-Bou et al., 2017; Edwards et al., 2019; Reitz and Mayeux, 2014).

Genetic risk factors also affect sporadic $A D$. Several genes have been identified as a risk factor for sporadic AD including TREM2, PLD3, ADAM10, CD2AP, DSG2, and $A P O E$ (Karch and Goate, 2015). Among these genes, APOE polymorphism has been one of the most widely studied risk factor. The APOE gene containing three variants $(\varepsilon 2, \varepsilon 3$ and $\varepsilon 4)$ represents the greatest risk for developing sporadic $A D$. Here, $\varepsilon 4$ is the main risk factor, as $\varepsilon 4$ heterozygotes have three-fold higher risk, which rises to twelve-fold in $\varepsilon 4$ homozygotes (Karch and Goate, 2015; Mahley, 2016). Given the low percentage of genetic causes, the identification of potential environmental risk factors for sporadic $A D$ is crucial.

Although age is considered a key risk factor for AD, several studies have also implicated chronic stress as a crucial environmental risk factor (Hoeijmakers et al., 2017; Huang et al., 2016; Islam et al., 2019; Justice, 2018; Lesuis et al., 2016; Ownby et al., 2006). It has been proposed that oxidative stress plays an important role in the initiation and progression of $A D$ (Wang et al., 2014). Oxidative stress is caused by an imbalance in the biochemical systems involved in the production and removal of reactive oxygen species (ROS). Reactive oxygen species are reactive molecules originated from oxygen (Andreyev et al., 2005), which are highly reactive due to presence of their unpaired electrons (Patten et al., 2010).

Several studies clearly indicate that an increased level of reactive oxygen species leads to oxidative stress and the manifestation of neurodegenerative disorders, including AD, ALS, and PD (Chen and Liu, 2017; Magalingam et al., 2018; Niedzielska et al., 2016; Patten et al., 2010). Oxidative stress can affect cell biology in many different ways, including damage to cell membranes and other functional units such as 
proteins, lipids, and DNA. In addition, oxidative stress may result in aberrant dynamics of SGs (Chen and Liu, 2017). The brain is particularly vulnerable to these insults because of its heightened demand for oxygen and its low antioxidant capacity (Chen et al., 2012; Cobley et al., 2018). Pathological and persistent SGs due to chronic stress and mutations in RBPs have been implicated in several neurodegenerative diseases (Liu-Yesucevitz et al., 2014; Vanderweyde et al., 2016). However, whether these RBP-pathological features are associated with rapid progression of the disease remains enigmatic.

\subsection{Atypical subtype of Alzheimer's disease}

Typically, sporadic $A D$ is characterized by a slow progression in cognitive decline, with conspicuous memory loss. Classically, disease duration spans $\sim 8$ years after the onset of clinical symptoms (Scheltens et al., 2016). However, emerging evidence shows heterogeneity in both clinical phenotypes and progression rates (AbuRumeileh et al., 2018; Ba et al., 2017; Cohen et al., 2015; Schmidt et al., 2011). These classical AD cases are abbreviated as SpAD in the current study.

Recently, a rapidly progressive variant of $A D$ ( $r p A D)$ has been described with a steep decline in the Mini-Mental State Examination (MMSE: a psychometric test) score (e.g. $\geq 6$ points/year), and/or a reduced survival duration ( $\sim$ years in comparison to 8 years for spAD cases) (Llorens et al., 2016; Nelson et al., 2009; Schmidt et al., 2010; Soto et al., 2008; Tosto et al., 2015). The prevalence and clinical definition of rpAD varies greatly across different studies in the literature. Preliminary evidence supports the notion that $\mathrm{rpAD}$ is associated with a specific molecular and pathogenic cascade (Ba et al., 2017; Cohen et al., 2015; Drummond et al., 2017; Schmidt et al., 2011). However, no significant differences have been detected in the core neuropathological features between SpAD and rpAD (Cohen et al., 2015; Schmidt et al., 2010), suggesting a great demand for a better understanding of molecular signatures responsible for this heterogeneity.

Rapidly progressive AD also exhibits significant clinical overlap with CreutzfeldtJakob disease, another rapidly progressive dementia, which makes early differential diagnosis a challenge. The rpAD often mimics the disease duration of CJD. Previous reports have also demonstrated similarity in biomarker profile and clinical features (rapid course, development of early focal neurological signs, levels of CSF markers 
14-3-3 and total tau) between rpAD and CJD (Abu-Rumeileh et al., 2017; Schmidt et al., 2011; Stoeck et al., 2014).

In fact, in prion disease referral centres worldwide, rpAD is a common non-prion diagnosis at neuropathological investigation, accounting for $\sim 14-50 \%$ of all non-CJD cases (Chitravas et al., 2011; Jansen et al., 2012; Lattanzio et al., 2017; Stoeck et al., 2012). The lack of understanding, how molecular mechanisms and risk factors lead to rapid progression seen in the various rapidly progressive forms of dementia (rpAD and sCJD), has hindered the development of therapeutic interventions, specifically at the early stages.

The most common human prion disease, CJD is a rapidly progressive, rare, transmissible and fatal disease, with patients exhibiting dementia among other major clinical symptoms (Collinge, 2001; Johnson and Gibbs, Jr., 1998; Prusiner, 1982; Zerr and Parchi, 2018). There are four major types of CJD: sporadic, familial, iatrogenic and the variant form. Sporadic CJD is the most prevalent form (85\%) of all human prion diseases (Parchi et al., 1999; Tschampa et al., 2007; Zerr and Parchi, 2018). Within SCJD, there are six distinct subtypes as determined by a combination of polymorphism at codon 129 of prion protein gene (PRNP) and two types of strains (type 1 and 2). The six subtypes have been classified as SCJD-MM1, -MV1, -VV1, -MM2, MV2 and -VV2 corresponding to different clinical phenotypes (Bishop et al., 2010; Parchi et al., 1999; Parchi et al., 2009). Among these subtypes, sCJD-MM1 and sCJD-VV2 subtypes are the most prevalent ones (Parchi et al., 1999; Meissner et al., 2009), therefore they were investigated in the present study along with ADsubtypes to uncover common molecular factors underlying variant progression rates.

During the last decades, there has been significant progress in understanding the risk factors and molecular basis underpinning $A D$. It has become clear that $A D$ is a multifaceted disease; multiple theories have been proposed, with the amyloidcascade hypothesis being the most studied one. The recent failure of many $A \beta$ oriented clinical trials has disappointed the field and led to a focus on other molecular mechanisms associated with this complex neurodegenerative disorder. Furthermore, recent discovery of various progression phenotypes of $A D$ demands a great need for understanding molecular factors, leading to heterogenous progression phenotypes in AD. Emerging evidence indicates an increasingly compelling role of dysfunctional neuronal RBPs and stress granules (Ramaswami et al., 2013, Wolozin, 
2012) in neurodegenerative diseases. To this end, this study aims to identify and characterize RNA-binding proteome (RBPome) alterations in subtypes of Alzheimer's and prion diseases, to open new avenues for early diagnosis and diseasemodifying therapies.

\subsection{Objectives of the study}

Based on previous observations, we hypothesize that global deregulation of RNARBP processes contributes to the pathophysiology of Alzheimer's and other neurodegenerative diseases, particularly prion diseases. RNA-binding proteins may represent a previously "hidden" component of pathophysiology of Alzheimer's and prion diseases. Understanding the global derangement of RBPs during the course of the disease will potentially unveil new targets for the design of therapeutics.

The present study was conducted to uncover pathological mechanisms linked to dysfunctional RBPs, leading to heterogeneous progression rates and phenotypes of AD. The study principally focusses on defining differential RBPome signatures in spAD, rpAD, two prion disease subtypes (sCJD-MM1 and SCJD-VV2) and age-matched controls. In the next part of the study, target proteomic candidates will be characterized in the human brain as well as cellular and animal models to explore the mechanistic role of these signatures in the pathogenesis and progression of the disease.

The objectives of the study were:

1. to identify and characterize RBPome signatures from diseased and healthy subjects, to have an overview of multiple deregulated pathways,

2. to perform a differential expressional analysis of target RBPs in the human brain and mouse brain model and at a cellular model,

3. to identify and characterize pathological mechanisms associated with target RBPs in the progression rate variations, and

4. to translate RBP signatures in cellular and animal models, to find a mechanistic link with the pathological features of the disease. 


\section{Materials and methods}

\subsection{Materials}

\subsubsection{Antibodies}

All the antibodies used for immunoblotting (IB) and immunofluorescence (IF) are listed in Tables 2 and 3.

Table 2: List of primary antibodies used in the study

\begin{tabular}{lllll}
\hline Primary Antibody & Origin & $\begin{array}{l}\text { Dilution } \\
\text { (IB) }\end{array}$ & $\begin{array}{l}\text { Dilution } \\
\text { (IF) }\end{array}$ & Cat. No./ Company \\
\hline Tau-5 & Mouse & $1: 500$ & $1: 100$ & ab80579/Abcam \\
Tau (E178) & Rabbit & $1: 1000$ & $1: 100$ & ab32057/Abcam \\
Anti-tau (T22), oligomeric & Rabbit & $1: 1000$ & $1: 250$ & ABN454/Sigma-Aldrich \\
Phospho-tau (S199) & Rabbit & $1: 1000$ & $1: 100$ & ab81268/Abcam \\
TIA-1 & Rabbit & $1: 500$ & $1: 100$ & ab140595/Abcam \\
TIA-1 & Mouse & $1: 500$ & - & ab40693/Abcam \\
TIA-1(G-3) & Rabbit & $1: 200$ & $1: 100$ & sc-166247/Santa Cruz \\
TIA-1 (G-3) AlexaFluor 488 & Mouse & - & $1: 50$ & sc-166247/Santa Cruz \\
SFPQ & Rabbit & $1: 500$ & $1: 100$ & ab38148/Abcam \\
VCP & Rabbit & $1: 3000$ & $1: 200$ & ab109240/Abcam \\
GAPDH & Mouse & $1: 3000$ & - & G8795/Sigma-Aldrich \\
B-Actin & Mouse & $1: 1000$ & - & ab8227/Abcam \\
BRD4 & Rabbit & $1: 1000$ & - & ab128874/Abcam \\
\hline
\end{tabular}

Table 3: List of secondary antibodies used in the study

\begin{tabular}{|c|c|c|c|c|}
\hline $\begin{array}{l}\text { Secondary } \\
\text { antibody }\end{array}$ & Origin & Dilution (IB) & Dilution (IF) & Cat. No. /Company \\
\hline a-Mouse-HRP (IgG) & Goat & $1: 10000$ & - & $\begin{array}{l}\text { 115-035-062/Jackson } \\
\text { IR Lab }\end{array}$ \\
\hline$\alpha$-Mouse-HRP (IgM) & Goat & 1: 10000 & - & $\begin{array}{l}\text { AP128P/Merck } \\
\text { Millipore }\end{array}$ \\
\hline$\alpha$-Rabbit-HRP (IgG) & Goat & 1: $10000 / 1: 5000$ & - & $\begin{array}{l}\text { 11-035-144/Jackson IR } \\
\text { Lab }\end{array}$ \\
\hline a-Mouse-A488 & Goat & - & $1: 200$ & A-11001 /Invitrogen \\
\hline a-Rabbit-A488 & Goat & - & $1: 200$ & A-11008/Invitrogen \\
\hline a-Mouse-A555 & Goat & - & $1: 200$ & A-21424/Invitrogen \\
\hline$\alpha$-Rabbit-A546 & Goat & - & $1: 200$ & A-11010/Invitrogen \\
\hline
\end{tabular}




\subsubsection{Antibiotics, enzymes and standards}

Table 4: All antibiotics, enzymes and standards used

Cat. No. / Company

\section{Antibiotics}

Ampicillin

171254, Calbiochem

Penicillin-Streptomycin (PS)

15140122, Thermo Fisher Scientific, Dreieich, Germany

\section{Enzymes}

Taq DNA Polymerase, $5 \mathrm{U} / \mu \mathrm{L}$

11146173001, Sigma-Aldrich, Deisenhofen, Germany

Standards (Protein and DNA)

Bovine serum albumin (BSA)

P0914, Sigma-Aldrich

DNA ladder

SM1333, Thermo Fischer Scientific

Precision Plus Protein Standard

161-0374, Bio-Rad, Munich, Germany

\subsubsection{Bacterial strain and culture media}

Table 5: List of bacterial strain and culture media

\begin{tabular}{ll}
\hline Bacterial Stain and media & Catalog No./Company \\
\hline E. coli strain DH5a & Addgene \\
LB medium & A0954/PanReacAppliChem ITW reagents \\
LB agar & A0949/ PanReacAppliChem ITW reagents \\
\hline
\end{tabular}

\subsubsection{Cell culture reagents}

Table 6: Reagents used in cell culture

\begin{tabular}{ll}
\hline Reagent & Catalog No./Company \\
\hline DMEM, high glucose, HEPES, no phenol red & $21063 /$ Thermo Fisher Scientific \\
Fetal bovine serum (FBS) & F7524/Sigma-Aldrich \\
Lipofectamine 2000 & $11668027 /$ Thermo Fisher Scientific \\
Opti-MEM, reduced Serum Medium, no phenol red & $11058021 /$ Thermo Fisher Scientific \\
Phosphate-buffered saline (PBS) & L1825/Merck \\
Trypsin/EDTA solution & T4174/Sigma-Aldrich \\
GlutaMAX supplement & Gibco 35050038/Thermo Fischer Scien- \\
\hline
\end{tabular}

\subsubsection{Chemicals}

All chemicals used in the present study were obtained from Sigma-Aldrich (Deisenhofen, Germany), Merck (Darmstadt, Germany), Roth (Karlsruhe, Germany), BioRad (Munich, Germany), Amersham (Freiburg, Germany), Fluka (Deisenhofen, 
Germany), Thermo Fisher Scientific (Darmstadt, Germany), or unless otherwise stated.

\subsubsection{Instruments and other materials}

Table 7: Instruments and appliances used in the study

\begin{tabular}{|c|c|c|}
\hline Appliances & Model/Description & Manufacturer \\
\hline $\begin{array}{l}\text { Centrifuges } \\
\text { C1000 Touch Thermal Cycler }\end{array}$ & $\begin{array}{l}5415 \mathrm{C} \\
\text { Optima TL } 100\end{array}$ & $\begin{array}{l}\text { Eppendorf, Hamburg, Germany } \\
\text { Beckman, Krefeld, Germany } \\
\text { Bio-Rad, USA }\end{array}$ \\
\hline ChemiDoc XRS+ system & $170-8265$ & Bio-Rad \\
\hline Electrophoresis apparatus & $\begin{array}{l}\text { Mini-ProteanSarstedt } \\
\text { III }\end{array}$ & Bio-Rad \\
\hline Filtopur V50 0.2 (Vacuum filter) & 83.1823 .001 & $\begin{array}{l}\text { SARSTEDT, Nümbrecht, Ger- } \\
\text { many }\end{array}$ \\
\hline Heated magnetic stirrer & iKAMAG RCT & $\begin{array}{l}\text { IKA-Labortechnik, Staufen, } \\
\text { Germany }\end{array}$ \\
\hline Ice machine & - & Ziegra, Isernhagen, Germany \\
\hline Incubator & IFE 400 & Memmert, Schwabach, Germany \\
\hline $\begin{array}{l}\text { Light Cycler } 480 \text { Multiwell Plate 96, } \\
\text { white }\end{array}$ & - & $\begin{array}{l}4729692001 \text {, Roche Life scienc- } \\
\text { es, Germany }\end{array}$ \\
\hline Microscope & Leica TCS SPE & $\begin{array}{l}\text { Leica Microsystems, Wetzlar, } \\
\text { Germany }\end{array}$ \\
\hline Microscope & Zeiss LSM 510 Meta & Carl Zeiss \\
\hline Microscope & $\begin{array}{l}\text { Zeiss } 667183 \text { Axio- } \\
\text { vert } 25 \mathrm{C}\end{array}$ & Carl Zeiss \\
\hline Microwave oven & ER-6320 PW & $\begin{array}{l}\text { Brother International, Bad Vilbel, } \\
\text { Germany }\end{array}$ \\
\hline Microplate reader & $\begin{array}{l}\text { Perkin Elmer Wallac } \\
1420 \text { Victor }\end{array}$ & GMI, USA \\
\hline Power supply & Power Pac 300 & Bio-Rad \\
\hline Safe-Lock tubes & $0.2,0.5,1.5$ and $2 \mathrm{ml}$ & Eppendorf \\
\hline Semi-Dry transfer Cell & $\begin{array}{l}\text { Transblot Turbo } \\
\text { transfer system }\end{array}$ & Bio-Rad \\
\hline $\begin{array}{l}\text { Serological pipettes } \\
\text { plastic tubes } \\
\text { pH meter }\end{array}$ & $\begin{array}{l}2,5,10,25 \mathrm{ml} \\
15 \text { and } 50 \mathrm{ml} \\
\mathrm{pH} 526\end{array}$ & $\begin{array}{l}\text { Sarstedt } \\
\text { WTW,Weilheim, Germany }\end{array}$ \\
\hline pH strips $(6.5-10)$ & 1.09543 .0001 & Merck Millipore, Germany \\
\hline Shakers & CERTOMAT R & $\begin{array}{l}\text { Sartorius, Göttingen, } \\
\text { Germany }\end{array}$ \\
\hline Spectrophotometers & EL808 & $\begin{array}{l}\text { Bioteck instruments, Winooski- } \\
\text { vermont, Germany }\end{array}$ \\
\hline Syringes BD Discardit & $2,5,20 \mathrm{ml}$ & Becton Dickinson, NJ, USA \\
\hline TC-plate 6 well, Cell+F & 83.3920 .300 & SARSTEDT \\
\hline TC Flask T75, Cell+vented Cap & 83.3911 .302 & SARSTEDT \\
\hline Thermomixer & 5436 & Eppendorf \\
\hline TissueLyser LT & 85600 & Qiagen, Hilden, Germany \\
\hline UV-transilluminator & $200 \times 200 \mathrm{~mm}$ & Bachofer, Reutlingen, Germany \\
\hline Vacuum drier & UNIVAPO $150 \mathrm{H}$ & $\begin{array}{l}\text { UNIEQUIP, Martinsried, Germa- } \\
\text { ny }\end{array}$ \\
\hline
\end{tabular}




\subsubsection{Kits}

Table 8: Kits used in the present study

\begin{tabular}{ll}
\hline Name & Cat No. / Company \\
\hline Purelink Genomic DNA isolation Kit & K182001, Invitrogen \\
RNeasy Plus Universal Mini Kit & 73404, Qiagen \\
HI Speed Plasmid Midi Kit & 12643, QIAGEN \\
Pierce Magnetic RNA-Protein Pull-Down Kit & 20164, Thermo Fisher Scientific \\
MTS Assay Kit (Cell Proliferation) (Colorimetric) & ab197010, Abcam \\
Chemiluminescent Nucleic Acid Detection Module Kit & Thermo Fisher Scientific \\
High-Capacity cDNA Reverse Transcription Kit & 4368814, Thermo Fisher Scientific \\
\hline
\end{tabular}

\subsubsection{Mammalian cell lines and culture media}

2.1.8.1 HeLa cells: HeLa cells were kindly provided by Dr. Aman-Deep Singh Arora, European Neuroscience Institute Göttingen, Georg-August University Göttingen, Germany. The cells were cultured in DMEM, supplemented with $10 \%$ FBS and $1 \%$ PS at $37^{\circ} \mathrm{C}$ with $5 \% \mathrm{CO}_{2}$ and $95 \%$ humidity.

2.1.8.2 SH-SY5Y cells: SH-SY5Y cells were obtained from Prof. Walter SchulzSchaeffer, Department of Neuropathology, University Medical Center (UMG), Göttingen, Germany. The cells were cultured in DMEM, supplemented with 10\% FBS, 1\% GlutaMax supplement and $1 \%$ PS at $37^{\circ} \mathrm{C}$ with $5 \% \mathrm{CO}_{2}$ and $95 \%$ humidity.

\subsubsection{Plasmids}

Plasmids for human wild-type tau (pRK5-EGFP-tau, cat. \#46904) and mutated tau (pRK5-EGFP-tau P301L, cat. \#46908) were purchased from Addgene (originally prepared by Karen Ash lab) (Hoover et al., 2010).

\subsubsection{Primer pairs}

All primers were purchased from Eurofins Genomics. List and sequences of primer pairs are provided in annexure data Table 14. 


\subsubsection{Software and online tools}

Table 9: List of software and web-based tools

\begin{tabular}{|c|c|c|}
\hline Name & Description/use & References \\
\hline GraphPad Prism (6.01) & Statistical analysis & $\begin{array}{l}\text { GraphPad Software, Inc. California, } \\
\text { USA }\end{array}$ \\
\hline catGRANULES & $\begin{array}{l}\text { Liquid-Liquid phase separation } \\
\text { property (LLPS) estimation }\end{array}$ & http: //s.tartaglialab.com \\
\hline $\begin{array}{l}\text { Functional enrichment } \\
\text { analysis tool (FunRich) }\end{array}$ & Functional enrichment analysis & http: //www.funrich.org/ \\
\hline FIJI 1.52p & Statistical analysis & National institutes of Health, USA \\
\hline Image J 1.51j8 & Immunofluorescence analysis & National institutes of Health, USA \\
\hline Image Lab (3.0.1) & Densitometric analysis & Kapelan, GmbH/Halle, Germany \\
\hline Inkscape (0.92) & $\begin{array}{l}\text { Professional quality vector } \\
\text { graphics software }\end{array}$ & https: //www.inkscape.org \\
\hline IPA & Pathway mapping & Qiagen, USA \\
\hline LAS $\mathrm{X}$ & Imaging software & $\begin{array}{l}\text { Leica Microsystems/Wetzlar, Ger- } \\
\text { many }\end{array}$ \\
\hline $\begin{array}{l}\text { Perseus software } \\
(1.5 .0 .31)\end{array}$ & Proteomics data analysis & $\begin{array}{l}\text { MPI of Biochemistry, Martinsried, } \\
\text { Germany }\end{array}$ \\
\hline PLAAC software & Prion-like domain scanning & http: //plaac.wi.mit.edu \\
\hline$R$ version 3.4 .3 & Statistical analysis & Proteome Software, Inc \\
\hline Cytoscape 3.6.1 & Protein network visualization & Cytoscape.Js \\
\hline Scaffold 4.8 .4 & MS/MS data analysis & Proteome Software, Inc \\
\hline $\begin{array}{l}\text { WEB-based GEne SeT } \\
\text { AnaLysis Toolkit } \\
\text { (WebGestalt) }\end{array}$ & Functional enrichment analysis & http: //www.webgestalt.org/ \\
\hline Zeiss LSM 4.2.0.121 & Immunofluorescence imaging & $\begin{array}{l}\text { Microimaging } \mathrm{GmbH} \text {, Göttingen, } \\
\text { Germany }\end{array}$ \\
\hline
\end{tabular}

\subsubsection{Solutions and buffers}

Note: $\mathrm{ddH}_{2} \mathrm{O}$ water was used to prepare solutions and buffers

Blocking solution for immunoblotting: 5\% non-fat dry milk in PBS-T/TBS-T

Cell-lysis buffer: 50 mM Tris- $\mathrm{HCl}, \mathrm{pH} 8,1 \%$ Triton X-100, 0.5\% CHAPS, 1 mM DTT Citrate buffer: $10 \mathrm{mM}$ sodium citrate $(\mathrm{pH} 6.0)$

Coomassie stain: $0.1 \%$ Coomassie blue R-250, 10\% acetic acid, 50\% methanol, $40 \% \mathrm{ddH}_{2} \mathrm{O}$

PBS-T buffer: PBS and Tween-20 (0.05\% Tween)

Resolving gel buffer: $1.5 \mathrm{M}$ Tris, $0.4 \%$ SDS, $\mathrm{pH} 8.8$

SDS-running buffer: $25 \mathrm{mM}$ Tris, $192 \mathrm{mM}$ glycine, $0.1 \%$ SDS, $\mathrm{pH} 8.3$

Stacking gel buffer: $0.5 \mathrm{M}$ Tris, $0.4 \%$ SDS, pH 6.8

TBS-T buffer: 50 mM Tris, 150 mM NaCL, 0.05\% Tween-20, pH 7.6 
Tissue lysis buffer: $7 \mathrm{M}$ urea, $2 \mathrm{M}$ thiourea, $4 \%$ CHAPS (freshly added $2 \%$ ampholytes, $1 \%$ DTT)

Transblot-buffer: 192 mM glycine, 10\% methanol, 25 mM Tris- $\mathrm{HCl}$, pH 8.3 


\subsection{Methods}

\subsubsection{Patient cohorts and sample processing}

Patient cohort processing, neuropathological examination and brain tissue collection for this study were all conducted as previously described (Krbot and Glatzel, 2018; Zafar et al., 2018; Zafar et. al., 2017). Briefly, postmortem brain material was obtained from patients after the approval of the local ethics committee at the University Medical Center, Göttingen, Germany. Frontal cortex samples from spAD, rpAD and age-matched controls were provided by the brain bank of the Institute of Neuropathology (HUB-ICO-IDIBELL Biobank) and Biobank of Hospital Clinic-IDIBAPS Spain, following the legislation (Ley de la Investigación Biomédica 2013 and Real DecretoBiobancos, 2014). Frontal cortex samples were obtained from patients with SCJD subtypes (MM1 and VV2), from the Department of Neurology at the University Medical Centre, Göttingen.

Cases strictly fulfilling the following inclusion criteria were included in rpAD cohort:

1. initial classification as prion disease based on rapidly progressive neurological and clinical parameters;

2. presence of $A D$ pathological features, i.e., higher Braak stages and CERAD stages;

3. exclusion of other rapidly progressive dementias (e.g. prion disease) and potential causes of rapid progression, e.g. vascular pathology, extensive Lewy body pathology, significant vascular disease, inflammation, stroke and tumors, as assessed by standard neuropathological examination, and

4. absence of familial $A D$, as evident by family history.

Patients of all ages fulfilling all the above-stated criteria were included in the study.

Tissue samples were processed following previously described protocols (Zafar et al., 2018). Briefly, whole brains were cut into two parts. One hemisphere was fixed with formalin (4\%), followed by treatment with formic acid. After fixation and decontamination, this hemisphere was stored at $-80^{\circ} \mathrm{C}$ until further use for neuropathological examination. Tissue sections of one-centimetre thickness were excised from the 
other hemisphere of each brain. Dissected tissues were immediately frozen and stored at $-80^{\circ} \mathrm{C}$ until used for biochemical investigations.

For immunohistochemistry, a separate cohort was used. Detailed description is given in annexure data table (Table 13). Samples were obtained from the University Medical Centre Hamburg-Eppendorf. Diagnosis of all these cases was confirmed neuropathologically by a combination of $A B C$ score, Thal staging for amyloid deposition (A), Braak staging for neurofibrillary tangles (NFTs) (B), and the Consortium to Establish a Registry for Alzheimer's Disease (CERAD) neuritic plaque score (C), as recommended by the current criteria of the National Institute on Aging, USA (NIA) for AD. Ethical approval was attained from the ethics committee of the University Medical Centre Göttingen, and all the procedures were followed in accordance to ethics regulations (Nr. 1/11/93 and Nr. 9/6/08).

\subsubsection{Pathological profiles}

Non-demented control cortical samples exhibited mild pathology (Braak stage I - II). Both spAD and rpAD samples had AD pathologies ranging from Braak stage III to VI. The samples for $A D$ subtypes were included without co-pathologies. Likewise, the SCJD subtypes (SCJD-MM1 and -VV2) cohort was exclusively composed of prionopathies. Details of the cohorts are described in the annexure data tables (Tables 11-13). No significant differences were observed in the age distribution and postmortem intervals (PMI) among the disease groups investigated in the study, as shown in Fig. 5 and 6.

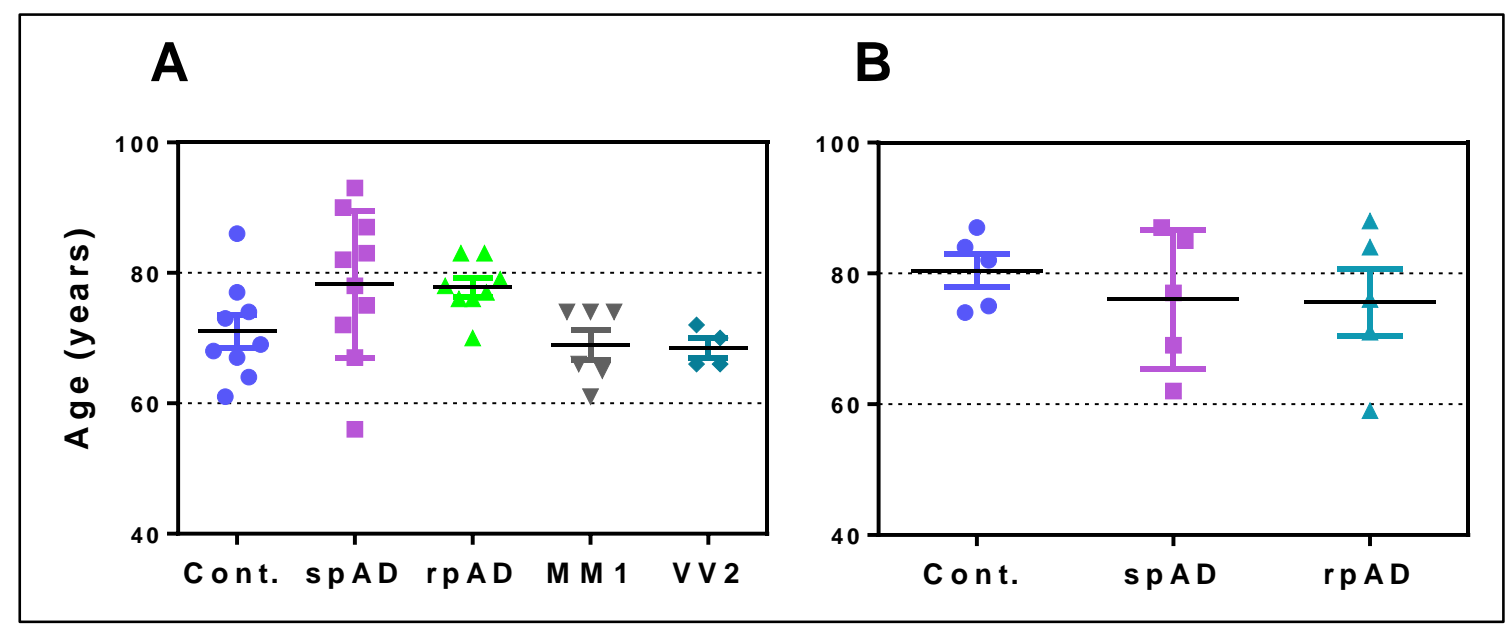


Figure 5: Patient cohorts included in the present study. A) Comparison of ages of the control and diseased subjects used for RBPome isolation, immunoblotting and qRT-PCR analysis. B) Comparison of ages in patient cohort used for immunohistochemical analysis.

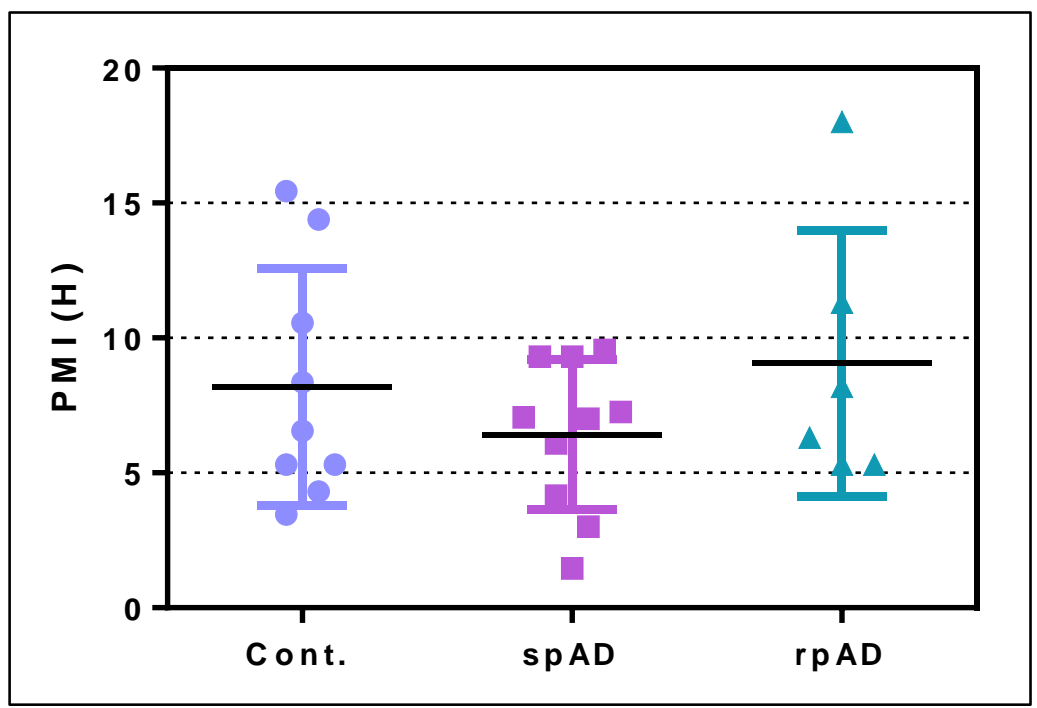

Figure 6: Comparison of postmortem intervals: Comparison of postmortem intervals to the time of autopsies from cases used in the present study.

\subsubsection{Molecular biology methods}

\subsubsection{Genomic DNA extraction}

Genomic DNA was isolated from human brain frontal cortex samples for APOE genotyping. DNA extraction was performed using the purelink genomic DNA isolation kits, in accordance with the manufacturer's protocols. Approximately $30 \mu \mathrm{g}$ of tissue was used for each extraction. Concentration estimation of DNA was performed with Nanodrop $\left(\mathrm{OD}_{260}=50 \mu \mathrm{g}\right.$ for dsDNA). Integrity of DNA was confirmed by agarose gel electrophoresis, followed by ethidium bromide staining.

\subsubsection{APOE genotyping}

The genotyping of the APOE polymorphism was performed using the APOE Strip Assay kit (GenoType APOE, Hain Lifesciences), as described previously (Al-Asmary et al., 2015). Briefly, the procedure involves two steps: the first involves polymerase chain reaction (PCR) amplification using biotinylate primers, followed by reverse hybridization of the amplified products on a test strip with allele specific oligonucleotides immobilized as an array in line. Biotinylated sequences bound to the strips were detected by streptavidin alkaline phosphatase, in order to develop color 
with color substrates. The frequency of $A P O E$ gene polymorphisms is represented for both spAD and rpAD cases in Fig. 7. An increasing trend was observed for alleles $3 / 3$ and $3 / 4$ in rpAD cases.

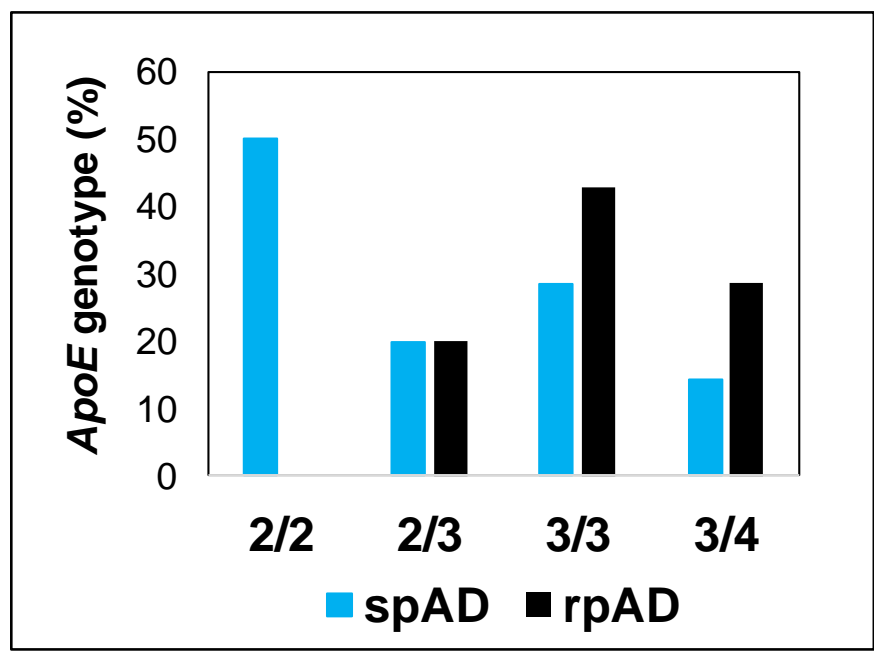

Figure 7: APOE genotype: The percentage of $A P O E$ genotype from slow progressive (spAD) and rapidly progressive Alzheimer's disease (rpAD) patients included in the present study.

\subsubsection{RNA extraction}

For the preparation of all reagents for RNA isolation, RNase-free water was used. Before RNA extraction, surfaces and lab-ware were cleaned with RNAseZap (Thermo Fischer Scientific). Total RNA was extracted from the human brain frontal cortices of diseased and control subjects using RNeasy Plus Universal kits (Qiagen, Germany), including DNase treatment in accordance with the manufacturer's instructions. RNA concentration was measured using a Nanodrop 2000 (Thermo Scientific) apparatus. Ratios of $A_{260} / A_{280}$ were also calculated for all samples. RNA integrity was confirmed by using a Bioanalyzer (Agilent Technologies, Santa Clara, CA). Samples bearing RNA integration number $(R I N) \geq 5$ were used for further analysis.

\subsubsection{Tissue lysis for pull-down assay}

Frontal cortex tissues from each subject were lysed in 'tissue protein extraction reagent (T-PER; Thermo Fischer Scientific), supplemented with phosphatase and protease-inhibitor cocktails (Roche, Germany). The concentration of isolated proteins was determined using Bradford assay (Bio-Rad). Total protein extract concentration 
was kept greater than $2 \mathrm{mg} / \mathrm{mL}$, such that there is significant dilution into the binding reaction buffer.

\subsubsection{RNA pull-down assay}

Total brain-derived RNA was end-labeled with desthiobiotin using T4-RNA ligase from Pierce RNA 3'-End Desthiobiotinylation Kit, which was a part of the Pierce magnetic RNA-protein pull-down kits (Thermo Fisher Scientific). The labelling efficiency of experimentally labelled samples was estimated by dot blotting (Thermo Scientific Chemiluminescent Detection Module, Product No. 89880), according to instructions from the manufacturer.

Labelled RNA was used for the enrichment of RBP complexes, according to the manufacturer's recommendations (Thermo Fischer Scientific). Briefly, experimentally labelled RNA was bound to $50 \mu \mathrm{L}$ of streptavidin magnetic beads in RNA capture buffer and incubated on a rotation wheel for $30 \mathrm{~min}$ at room temperature (RT). Beads were washed three times with $20 \mathrm{mM}$ Tris ( $\mathrm{pH}$ 7.5). Bead-bound RNA was incubated with total protein extract, which was isolated from the human brain frontal cortex, for 1 hour (hr) in RNA-protein binding reaction buffer at $4^{\circ} \mathrm{C}$ followed by another three washes. Bound protein complexes were eluted with biotin elution buffer and processed for mass spectrometry (MS) analysis. In this assay, streptavidin magnetic beads were mixed with protein extract in the absence of biotinylated transcript as a control for nonspecific binding.

\subsubsection{Label-free quantification mass spectrometry (LFQ-MS) analysis}

Mass spectrometry analysis was carried out as published previously (Zafar et al., 2017). Briefly, isolated protein complexes were separated by $4-20 \%$ Bis-Tris gels (NuPAGE Novex Bis-Tris Mini gels, Invitrogen) for a length of $\sim 1 \mathrm{~cm}$ followed by Coomassie staining. The bands were excised from the gel into small slices (1-2 $\mathrm{mm}^{2}$ ). The gel pieces were initially rinsed with $\mathrm{ddH}_{2} \mathrm{O}$ followed by reduction $(10 \mathrm{mM}$ dithiothreitol [DTT] for $30 \mathrm{~min}$ at $56^{\circ} \mathrm{C}$ ) and alkylation ( $55 \mathrm{mM}$ iodoacetamide [IAA] at $\mathrm{RT}$ in dark for $1 \mathrm{hr}$ ). Then, the gel slices were washed with acetonitrile (ACN) for 15 min and dried using a SpeedVac. The gel slices were incubated overnight with the minimum possible amount of trypsin $(12.5 \mathrm{ng} / \mu \mathrm{L}$ in $0.025 \mathrm{M}$ aqueous ammonium bicarbonate) at $37^{\circ} \mathrm{C}$. After digestion with trypsin, $\mathrm{ddH}_{2} \mathrm{O}(10 \mu \mathrm{L})$ was added to the 
slices for $15 \mathrm{~min}$ at $37^{\circ} \mathrm{C}$, followed by the addition of $\mathrm{ACN}(80 \mu \mathrm{L})$ for $15 \mathrm{~min}$ at $37^{\circ} \mathrm{C}$. Supernatant was isolated after a short-spin. Residual peptides were recovered from slices by incubation for $15 \mathrm{~min}$ at $37^{\circ} \mathrm{C}$ with $5 \%$ formic acid (FA) $(65 \mu \mathrm{L})$. Again, ACN $(65 \mu \mathrm{L})$ was added for $15 \mathrm{~min}$ at $37^{\circ} \mathrm{C}$. Supernatant from this step was collected and added to previous supernatant. Total volume of supernatant was evaporated in a vacuum concentrator to dry. These samples were suspended in $\mathrm{ACN}(30 \%, 10 \mathrm{uL})$ and triflouroacetic acid (TFA, $0.1 \%$ ).

For MS measurements, the peptide mixture was concentrated on a Reversed PhaseC18 precolumn ( $0.15 \mathrm{~mm}$ ID $\times 20$ mm, self-packed with Reprosil-Pur 120 C18-AQ 3 $\mu \mathrm{m}$ material), followed by separation using Reversed Phase-C18 nanoflow chromatography on a Picofrit column, $0.075 \mathrm{~mm}$ ID $\times 200 \mathrm{~mm}$ (New Objective, Woburn, USA) and a 15 min linear gradient on an Easy nLC-1000 nanoflow chromatographic system (Thermo Fisher Scientific). Samples were measured on a Q-Exactive hybrid quadrupole/orbitrap MS system operated under Excalibur v2.4 software (Thermo Fisher Scientific).

Analysis was performed by a Top10 method in the Data Dependent Acquisition mode. Tandem mass spectra were extracted for database searching using the Raw2MSM v1.17 software (Max Planck Institute for Biochemistry, Martinsried, Germany). MS/MS samples were analyzed using the Mascot (Matrix Science, London, UK; version 2.5.1) set for searching UniProt/SwissProt database (release 02/17 filtered for Homo sapiens, 92928 entries). Mascot was searched with a fragment ion mass tolerance of $0.050 \mathrm{Da}$ and a parent-ion tolerance of 10.0 PPM. Carbamidomethyl of cysteine was specified in Mascot as a fixed modification. Oxidation of methionine, acetylation of the $\mathrm{N}$-terminus and phosphorylation of serine, threonine and tyrosine were specified in Mascot as variable modifications and 2 missed cleavages were allowed. Scaffold (version Scaffold 4.8.4, Proteome Software Inc., Portland/OR, USA) was used to validate MS/MS-based peptide and protein identifications. Peptide identifications were accepted if they could be established at greater than $95 \%$ confidence. For protein identifications, a minimum of two peptide counts and a confidence threshold of $99 \%$ was used. Protein probabilities were assigned by the Protein Prophet algorithm, as described by Nesvizhskii et al. (2003). Proteins sharing similar peptide sequences were classified into defined clusters. 


\subsubsection{Differential enrichment analysis of RBPome}

For a detailed analysis of the isolated proteome, three different approaches were used, here labelled approach A, B and C. Approach A was used to identify proteins differentially enriched in the various disease groups. Pairwise $t$-tests (two-sided) were performed for all disease group combinations using Perseus software (version 1.5.0.31) (Tyanova and Cox, 2018), with a p-value $<0.05$ and multiple testing correction using the Benjamini-Hochberg $(\mathrm{BH})$ method. Using label-free MS to identify and quantify proteins, zero values were observed in the data for several proteins. Missing or zero values appeared when the mass spectrometer could not detect peptides having abundances below the censoring cutoff of the mass spectrometer. Such values were informative because they were below the lowest abundance observed for a peptide. In such situations, when quantitative values were missing in one group but were present in other groups, this more likely represented differences in abundance between groups, which might indicate interesting features specific to that group. Therefore, zero values were imputed by half of the minimum value of total spectrum count values, to have statistical analysis of the proteomic candidates (Approach A). Fold change (FC) for all comparisons' thresholds was set at \pm 1.5 and a $p$-value < 0.05 for significance. Proteins which were identified as significantly enriched were used to make heatmaps using Perseus software (version 1.5.0.31). Volcano plots were also calculated using Perseus software, where FC was $\log _{2}$ transformed, so that the data were centered on zero, while the $p$-values were transformed into - $\log _{10}$.

In addition, approach B was used. Given the exploratory nature of this discoverybased proteomic work-flow, the criteria were relaxed and proteins with a single quantitative value were included in the analysis to compare all the disease groups. Venn diagrams were prepared, showing similar and unique proteins with functional enrichment and analysis tool (FunRich).

To have a deeper proteome coverage of isolated RNA-binding proteome of particularly rapidly progressive forms of dementia (rpAD, SCJD-MM1 and SCJD-VV2 subtypes), we used SWATH-MS quantitative proteomic analysis as the approach $\mathrm{C}$, which also served as a confirmatory method for the LFQ-MS analysis. Detailed description of SWATH-MS is described in Section 2.2.15.8. Quantified proteins were analysed using Perseus software (version 1.5.0.31), to identify significantly modulat- 
ed proteins between two pairs of comparisons, namely rpAD vs SCJD-MM1 and rpAD vs sCJD-VV2. Significantly abundant proteins were visualized by heatmaps generated using Perseus software (version 1.5.0.31).

\subsubsection{Quantitative real-time PCR (qRT-PCR)}

Complementary DNA (cDNA) was synthesized from brain-derived total RNA $(1 \mu \mathrm{g})$, using the High Capacity cDNA Reverse Transcription Kit (Thermo Fisher Scientific). The synthesis was performed according to the instructions from the kit protocol. The resulting $\mathrm{CDNA}$ was diluted $(1: 10)$ and stored at $-20^{\circ} \mathrm{C}$. All primer pairs for qRT-PCR assays were designed with Primer 3 . The product size of all the primers was in a range of 100 to 150 base pair long. The primer sequences were tested using NCBI/Primer BLAST and synthesized by Eurofins Genomics (Table 14). Reaction mixtures for RT-PCR were prepared by mixing $1 \mathrm{uL}$ of $10 \mathrm{x}$ PCR reaction buffer (Roche), $1 \mathrm{uL}$ of dimethylsulphoxide (DMSO) (Sigma), $0.5 \mathrm{uL}$ of 1:1000 dilutions of SYBR Green (Sigma), $0.2 \mathrm{mmol}$ of each dNTP (Roche), 0.15 units of Taqpolymerase, $10 \mathrm{pm} / \mathrm{uL}$ of forward and reverse primers and $1 \mathrm{uL}$ of CDNA (1:10 diluted); the volume was filled up to $20 \mathrm{uL}$ with RNAse-free water. Amplification was performed using Light Cycler 480 (Roche), with an initial denaturation at $95^{\circ} \mathrm{C}$ for $2 \mathrm{~min}$, followed by $40-\mathrm{PCR}$ cycles (denaturation at $95^{\circ} \mathrm{C}$ for 30 seconds [sec], annealing at $56^{\circ} \mathrm{C}$ for $30 \mathrm{sec}$, and extension at $72^{\circ} \mathrm{C}$ for $30 \mathrm{sec}$ ). Reactions were done in triplicates. No template controls were used to ensure reaction specificity. The data were analysed with Light Cycler 480 software SW1.5.1 (Roche) and values were normalised to $G A P D H$. The comparative $\mathrm{C}_{t}$ method $\left(2^{-\Delta \Delta C t}\right)$ was used to measure relative fold change mRNA expression (Livak and Schmittgen, 2001).

\subsubsection{Preparation of brain homogenates for protein analysis}

Frontal cortex tissues were lysed from spAD, rpAD, sCJD (MM1 and VV2 subtypes) and non-demented control subjects, along with cortical tissues from mouse brains, as published previously (Zafar et al., 2018). Briefly, brain tissues were homogenized $(10 \%$, wt/vol) using tissue lyser LT (Qiagen) in ice-cold tissue lysis buffer containing protease and phosphatase inhibitors (Roche). To remove insoluble debris, lysates were centrifuged at $14000 \mathrm{rpm}$ for $30 \mathrm{~min}$ at $4^{\circ} \mathrm{C}$. Protein quantification of isolated extracts was performed by Bradford protein assay. Bradford assay dye reagent was diluted with $\mathrm{ddH}_{2} \mathrm{O}$ at a ratio of 1:5. Dilutions of protein standard, bovine serum al- 
bumin (BSA) were prepared $(0.0-1.0 \mathrm{mg} / \mathrm{mL})$ in $\mathrm{ddH}_{2} \mathrm{O}$. Total protein extracts from experimental samples were diluted at a ratio of 1:20. From this dilution, $20 \mu \mathrm{L}$ was mixed with $980 \mathrm{uL}$ of Bradford working solution and the mixture was incubated for 10 min at RT. All dilutions of protein standard and experimental samples were measured at a wavelength of $595 \mathrm{~nm}$. Measured optical density values were used for calculation of protein concentration.

\subsubsection{Immunoblotting}

Equal amounts of proteins (30-50 ug) from each brain lysate or cell lysate were resolved by molecular weight on 12\% SDS-PAGE polyacrylamide gels (prepared in house) or $4-12 \%$ Bis-Tris gels (NuPAGE $4-12 \%$ Bis-Tris Protein Gels, Invitrogen). The protein marker (Precision plus protein dual color standards from Bio-Rad) was used to visualize the correct separation of the proteins and to confirm the correct protein band sizes. After separation on the gel, proteins were transferred onto a polyvinylidene difluoride (PVDF) membrane with a $0.45 \mu \mathrm{m}$ pore size using a semi-dry blot chamber (Bio-Rad, Hercules, USA) for $1 \mathrm{hr}$. The membranes were blocked for 1 $\mathrm{hr}$ at RT in blocking reagent (5\% non-fat dry milk in TBS-T or PBS-T), followed by incubation with primary antibodies at $4^{\circ} \mathrm{C}$ overnight. All the primary antibodies (total tau, phospho-tau (S199), SFPQ, TIA-1, VCP, $\beta$-actin, BRD-4, and GAPDH) were diluted in blocking buffer. The dilutions of all the primary antibodies are given in Table 2. Next, the membranes were washed in PBS-T/TBS-T and incubated with secondary antibodies coupled to horseradish peroxidase (HRP) for $60 \mathrm{~min}$ at RT. Protein bands were detected using the enhanced chemiluminescent (ECL) method with Chemi-Doc (Bio-Rad). The densitometric analysis was performed with Image Lab software (3.0.1).

\subsubsection{Immunohistochemistry}

Formalin-fixed and paraffin-embedded human brain cortical sections ( $5 \mu \mathrm{m}$ thick) from patients with spAD and rpAD as well as non-demented controls were analysed by co-immunofluorescence using protocols validated previously (Krasemann et al., 2017; Vanderweyde et el., 2012). Briefly, tissue sections were deparaffinized using xylene, rehydrated and then proceeded for antigen retrieval with citrate buffer $(\mathrm{pH}$ 6.0) for $1 \mathrm{hr}$ at $95^{\circ} \mathrm{C}$. The slides were cooled for $30 \mathrm{~min}$ at RT and then washed with PBS for $1 \mathrm{hr}$ with repeated changes. Next, the sections were permeabilized with 
$0.2 \%$ Triton $X-100$ for 10 min, followed by two washes with PBS. The slides were then exposed to UV-A and UV-B for $20 \mathrm{~min}$, to remove background fluorescence. The sections were then blocked with a blocking reagent (1\% BSA, 10\% FBS in PBS) for $1 \mathrm{hr}$ at RT, treated with Sudan black to remove lipofuscin fluorescence and then washed with PBS for $30 \mathrm{~min}$. The primary antibodies specific for SFPQ, TIA-1, phospho-tau (S199) and oligomeric tau (T22) were incubated overnight at $4^{\circ} \mathrm{C}$. Dilutions of all the primary antibodies are given in Table 2. After incubation, the slides were washed in PBS for $1 \mathrm{hr}$. The secondary antibodies, Alexa488, Alexa546 and Alexa555 (Invitrogen), were incubated for $2 \mathrm{hrs}$ at RT followed by counter staining with TO-PRO-3 iodide for $1 \mathrm{~min}$ to visualize cell nuclei. After washing with PBS, the sections were mounted with Fluoromount-G (Thermo Fischer Scientific). After secondary antibody incubation, the whole procedure was carried out in dark.

\subsubsection{Confocal laser scanning and image analysis}

Imaging was performed with the confocal laser scanning microscope TCS-SPE from Leica, using 543 and $633 \mathrm{~nm}$ helium-neon and $488 \mathrm{~nm}$ argon excitation wavelengths. All images were separately analysed for co-localization using ImageJ (WCIF plugin) and FIJI software. Threshold Mander's overlap coefficient (tM) and Pearson's linear correlation coefficient (rP) were calculated to measure the strength and direction of linear correlations between two fluorescence channels. In addition, intensity correlation analysis (ICA) was performed. This analysis generated scatter-plots of channel $A$ and channel $B$ against the product of the difference of each pixel $A$ and $B$ intensities from their respective means (PDM) (Li et al., 2004). The resulting plots emphasized the high intensity stained pixels and allowed to identify protein pairs that vary in synchrony (positive PDM values), randomly (around 0), or independently (negative PDM values) within the cell.

\subsubsection{Microbiological methods}

\subsubsection{Culturing and storage of E. coli}

Plasmids were purchased from Addgene in the E.coli strain DH5a in agar stab and were propagated in-house. For long-term storage, bacterial strains were mixed with an equal volume of glycerol $(50 \%)$ and stored at $-80^{\circ} \mathrm{C}$. 


\subsubsection{Extraction of plasmid DNA}

For the extraction of plasmid DNA, Hispeed Plasmid Midi Kit (Qiagen) was used. A single bacterial colony was inoculated into $5 \mathrm{~mL}$ of LB medium containing ampicillin $(100 \mu \mathrm{g} / \mathrm{mL})$ followed by incubation for 12-16 hrs with continuous shaking (180-250 rpm) at $37^{\circ} \mathrm{C}$. The resultant bacterial pellets were harvested by centrifugation at $5,000 \times$ for $10 \mathrm{~min}$ at $4^{\circ} \mathrm{C}$. All further procedures were performed according to the instructions of the kit protocol.

\subsubsection{Cell biology methods}

\subsubsection{Cryopreservation of mammalian cell lines}

In order to be stored for longer, the cells were preserved in liquid nitrogen in DMSO. Cells at confluency (70-90\%) were harvested and centrifuged at $400 \times \mathrm{g}$ for $5 \mathrm{~min}$. The cell pellet was resuspended in freezing medium (5\% DMSO in culture medium) and immediately transferred to $1 \mathrm{~mL}$ cryogenic vials, followed by overnight freezing at $-80^{\circ} \mathrm{C}$. Finally, the vials were transferred to liquid nitrogen.

\subsubsection{Cell culturing and maintenance}

The cells were taken from the liquid nitrogen tank and incubated for 2 min in a $37^{\circ} \mathrm{C}$ water bath until nearly $80 \%$ thawed. The cells were then gently mixed with $10 \mathrm{~mL}$ of pre-warmed culture medium (DMEM, 10\% FBS, 1\% PS) and centrifuged at 400xg for $5 \mathrm{~min}$. The supernatant was removed and the pellet was suspended in $14 \mathrm{~mL}$ of the culture medium. The cells were cultured in flasks and plates at $37^{\circ} \mathrm{C}, 95 \%$ humidity and $5 \% \mathrm{CO}_{2}$. After $24 \mathrm{hrs}$, the media was replaced with a fresh $14 \mathrm{~mL}$ culture medium. Once the cells reached confluency, they were washed once with PBS followed by trypsinization using 2-3 $\mathrm{mL}$ of trypsin/EDTA solution. After trypsinization, cells were centrifuged at $400 x g$ for 5 min and then washed with PBS. Cell counting was performed using a haemocytometer $(0.1 \mathrm{~mm}$ sample depth). The cell pellet was resuspended in a fresh pre-warmed culture medium and cells were grown to approximately $70-90 \%$ confluency. All cell lines were cultured between 5 and 20 passages.

\subsubsection{Stress model for stress induction}

Confluent cells (HeLa and SH-SY5Y) were subjected to oxidative stress by adding a pre-warmed culture medium containing sodium arsenite $(0.6 \mathrm{mM})$ for $1 \mathrm{hr}$ at $37^{\circ} \mathrm{C}$. 
After $1 \mathrm{hr}$ of treatment, the cells were either fixed with $4 \%$ paraformaldehyde (PFA) for immunocytochemistry or processed for immunoblotting analysis.

\subsubsection{Immunocytochemistry}

The cells were grown in T75 flasks with culture medium (DMEM, 10\% FBS, 1\% PS). At confluency $(70-90 \%)$, cells were trypsinized and seeded $\left(5 \times 10^{4}\right)$ on glass coverslips $(13 \mathrm{~mm})$ in 24-well plates. Following the stress treatment as described above, the cells were fixed with 4\% PFA for 20 min at RT, followed by three washes with ice-cold PBS for 5 min each. Permeabilization was achieved using $0.2 \%$ Triton X-100 in PBS for 10 min, followed by three washes with PBS for 5 min each. To avoid non-specific binding, cells were incubated with blocking buffer (1\% BSA, 10\% FBS in PBS) for 30 min at RT. The primary antibody and, in case of double labelling both primary antibodies, were diluted in 1\% BSA in PBS, followed by overnight incubation at $4{ }^{\circ} \mathrm{C}$. The cells were washed three times with PBS (each wash was for 5 min) followed by incubation with secondary antibodies (see Table 3 ) diluted in $1 \%$ BSA in PBS for 2 hrs at RT in the dark. The cells were rinsed three times with PBS for $5 \mathrm{~min}$ each in the dark to remove non-specific immunoreactivity. The cells were then counterstained with DAPI for 1 min or a RedDot 2 Far red nuclear stain for 20 min. After nuclear staining, the cells were washed three times with PBS, followed by mounting with one drop of mounting medium (immuo-mount, Thermo Fisher Scientific). The slides were stored in the dark at $-20^{\circ} \mathrm{C}$ or $+4^{\circ} \mathrm{C}$ until they were imaged. The average number of stress granules in each cell was calculated using FIJI software.

\subsubsection{Subcellular fractionation}

Cellular fractionation after stress treatment was carried out as described previously (Suzuki et al., 2010). Briefly, confluent HeLa cells (70-90\%) were washed once with ice-cold PBS, scraped and resuspended in $1 \mathrm{~mL}$ of PBS. After a short spin (10 sec), the supernatant was removed and the cell pellet was resuspended in $900 \mu \mathrm{L}$ of the lysis buffer (0.1\% NP40 in PBS) (Calbiochem, CA, USA) followed by trituration (5x) using a p1000 micropipette. From this step, $300 \mathrm{uL}$ of the whole cell lysate fraction were isolated and mixed with $4 \mathrm{x}$ Laemmli buffer $(100 \mu \mathrm{L})$. The remaining $(600 \mu \mathrm{L})$ lysate was centrifuged for $10 \mathrm{sec}$. The supernatant $(300 \mu \mathrm{L})$ from this step was separated as the "cytosolic fraction" and mixed with $4 \mathrm{x}$ Laemmli buffer (100 $\mu \mathrm{L})$ followed 
by boiling for one min. The remaining supernatant in the original tube was removed and the pellet was resuspended in $1 \mathrm{~mL}$ of lysis buffer and centrifuged for $10 \mathrm{sec}$. This supernatant was removed and the pellet $(\sim 20 \mu \mathrm{L})$ was resuspended in $180 \mu \mathrm{L}$ of $1 x$ Laemmli buffer and saved as the "nuclear fraction". Whole-cell nuclear fractions containing DNA were sonicated in an ultrasonicator (Level 2, twice for $5 \mathrm{sec}$ ), followed by boiling for $1 \mathrm{~min}$. From each fraction, $10 \mu \mathrm{L}$ were proceeded for immunoblotting analysis with primary antibodies specific for total tau (tau-5), phospho-tau (S199), TIA-1, SFPQ, $\beta$-actin, BRD4, and GAPDH.

\subsubsection{Cell lysis and protein extraction}

Total protein extracts were prepared from $70-90 \%$ confluent HeLa and SH-SY5Y cells. After stress treatment, all the cell lines were washed with $1 \times$ PBS, scraped and resuspended in lysis buffer $(50 \mathrm{mM}$ Tris- $\mathrm{HCl}, \mathrm{pH} 8,1 \%$ Triton $\mathrm{X}-100,0.5 \%$ CHAPS, $1 \mathrm{mM}$ DTT, protease, and phosphatase inhibitors). Lysates were sonicated using an ultrasonicator on ice, followed by incubation for $1 \mathrm{hr}$ at $4^{\circ} \mathrm{C}$ with shaking. The lysates were centrifuged at $14000 \mathrm{rpm}$ for $30 \mathrm{~min}$ at $4^{\circ} \mathrm{C}$. The supernatants were transferred to new tubes and proteins were quantified as described above. To harvest cells after transient transfections, cells were trypsinized and washed with PBS followed by centrifugation at $400 \mathrm{xg}$ for $5 \mathrm{~min}$ at $4^{\circ} \mathrm{C}$. The washed cell pellets were lysed, and protein quantification was performed as above.

\subsubsection{Tau transfections}

Plasmids for human wild-type tau (pRK5-EGFP-tau) and mutated tau (pRK5-EGFPtau $\mathrm{P} 301 \mathrm{~L}$ ) were obtained from Addgene. The plasmids were propagated in E. coli DH5 $\alpha$ strain and extracted from the the transformed bacteria using the Hispeed Plasmid Midi kit (Qiagen). Transient transfections were achieved using the Lipofectamine 2000 (Invitrogen) reagent, according to the instructions of the manufacturer. HeLa cells (2x 105/well) were plated in 6-well plates and cultured for $24 \mathrm{hrs}$ in culture medium. The cells were washed with Opti-MEM I, followed by transfection with $2 \mu \mathrm{g}$ of DNA/well in the Opti-MEM I medium. The cells were collected from cultures after 24 and 48 hrs post-transfection. 


\subsubsection{SWATH-MS for global proteomics}

\subsubsection{Library preparation}

Analytical-grade reagents were used for protein extraction and digestion. Sterile water (Ampuwa, Fresenius, Bad Homberg, Germany) was used to prepare all buffers and solutions. For the preparation of the spectral peptide library, digested protein extracts (normalized for protein amounts) from each sample were pooled to a total amount of $220 \mu \mathrm{g}$ and separated into fourteen staggered pooled fractions, using an Äkta pure (GE Healthcare) with a Hypersil Gold C18 column (diameter 150x2,1 mm, Particle size: $3 \mu \mathrm{m}$ ). Protein digests were analyzed on an Eksigent nanoLC425 nanoflow chromatography system associated with TripleTOF $5600^{+}$, a hybrid triple quadrupole TOF mass spectrometer with a Nanospray III ionization source (ionspray voltage $2400 \mathrm{~V}$, Interface heater temperature $150^{\circ} \mathrm{C}$, Sheath gas setting 12). The peptides were dissolved in loading buffer $(0.1 \%$ formic acid, $2 \%$ acetonitrile in optima water [Thermo Scientific]) to a final concentration of $0.3 \mu \mathrm{g} / \mu \mathrm{L}$ and spiked with a synthetic peptide standard used for retention-time alignment (iRT Standard, Schlieren, Schweiz). For every measurement, digested proteins $(1.5 \mu \mathrm{g})$ were enriched on a precolumn (PharmFluidics $\mu$ PAC Trapping Column) and separated on a PharmaFluidics $\mu$ PAC microchip-based separation analytical column with $50 \mathrm{~cm}$ length, using a 120 min linear gradient of $5 \%-45 \%$ ACN, $0.1 \%$ FA at a flow rate of $300 \mathrm{~nL} / \mathrm{min}$. Qualitative LC-MS/MS measurement was carried out using a Top 20 data-dependent acquisition (DDA) mode with a mass range of $\mathrm{m} / \mathrm{z} 350-1250$ for 250 milliseconds (ms). The resolution for data extraction was 30,000 full width at half maximum (FWHM). MS/MS scans of mass range $m / z 180-1600$ at a resolution of 17,500 FWHM for $85 \mathrm{~ms}$ and a precursor isolation width of 0.7 FWHM with a cycle time of $2.9 \mathrm{sec}$ were used. For MS/MS, the criteria for precursor ions selection were as follows: a threshold intensity of more than $125 \mathrm{cps}$ with charge states of 2+, 3+, and 4+, and the dynamic exclusion of $30 \mathrm{sec}$. In every reversed-phase fraction, two technical replicates were analyzed for the construction of the spectral library.

\subsubsection{Quantitative SWATH measurement}

For the SWATH measurement, MS/MS data were obtained using windows of 65 variable sizes across a mass range of $400-900 \mathrm{~m} / \mathrm{z}$. For each biological sample, two technical replicate injections were attained. Protein identifications were attained us- 
ing ProteinPilot Software version 5.0 build 4769 (AB Sciex) at "thorough" settings. Spectra from MS/MS were searched in the UniProtKB using Homo sapiens as reference proteome (revision 04/2018, 93661 entries) at a critical false discovery rate (FDR) of $1 \%$. Spectral library and SWATH peak extraction were attained in PeakView Software version 2.1 build 11041 (AB Sciex). Following retention time correction using the iRT standard, peak areas were extracted using information from the MS/MS library at $1 \%$ FDR. The peak areas were summed up to peptide and finally corresponding protein area values that were used for downstream statistical and functional analysis.

\subsubsection{Gene Ontology analysis and functional network mapping}

To gain functional insights from proteomics data, three different enrichment strategies were used. Functional enrichment analysis was initially performed using Perseus software for significantly enriched Gene Ontology (GO) processes, including the Biological Process and Molecular Function by Fisher's exact test. Then, overrepresentation enrichment analysis was performed using the web-based Gene SeT Analysis Toolkit (WebGestalt), in the domains of Biological and Molecular Functions to have a GO Slim summary of enriched terms. Finally, an Ingenuity Pathway Analysis (IPA, Qiagen, USA) was performed to find out canonical pathways associated with up- and down-regulated proteins after tau expression, as described below.

\subsubsection{Ingenuity Pathway Analysis (IPA)}

The proteomic dataset containing the fold change and $p$-values of significantly regulated proteins was uploaded to IPA for core analysis (Qiagen, USA). The protein candidates from the submitted dataset generated top molecular networks based on Molecular and Biological Functions including canonical pathways, potential upstream regulators, and disease-based networks. The settings for analysis were based on direct and indirect relationships between differentially expressed proteins (DEPs), and these were supported by experimentally reported data from human, mouse and rat studies (Everts et al., 2014). Potential upstream regulators were designated as inhibited or activated, according to the fold change and $p$-values (- $\log _{10}-p$-values) (Sardiu et al., 2009) of the DEPs. In order to understand the complex relationships among significant disease-based networks, Cytoscape (3.6.1) was used to construct and visualize the networks. 


\subsubsection{Biochemical methods}

\subsubsection{MTS assay}

Cells were grown in T75 flasks with culture medium (DMEM, 10\% FBS, 1\% PS). At confluency (70-90\%), cells were trypsinized and then washed once with PBS. The cells were seeded $\left(10 \times 10^{3}\right)$ in 96 -well plates and incubated for $18-24 \mathrm{hrs}$ at $37^{\circ} \mathrm{C}$. Transfections were performed as described above with plasmids coding for wild-type tau and P301L-tau for variable periods of time (24 and $48 \mathrm{hrs}$ ). To measure cell viability, MTS assay (ab197010, Abcam) was used according to the manufacturer's protocol. The culture media was replaced with a fresh medium before treatment with the 3-(4,5-dimethylthiazol-2-yl)-5-(3-carboxymethoxyphenyl)-2-(4-sulfophenyl)-2H tetrazolium MTS reagent. To estimate the effect of tau expression on cell viability, the reduced MTS tetrazolium complex (colored formazan product) was measured. This conversion is a property of metabolically active cells. For color development, cells were incubated at $37^{\circ} \mathrm{C}$ for $1 \mathrm{hr}$ and the absorbance measurement was taken at 490 nm using a Perkin Elmer Wallac 1420 Victor microplate reader (GMI, USA). Absorbance from the control wells (background) was subtracted from the sample wells.

\subsubsection{Trypan blue exclusion assay}

Cell viability was also assessed through the trypan blue exclusion dye test. Briefly, the cell suspension in the culture medium $(25 \mu \mathrm{L})$ after trypsinization was mixed with $25 \mu \mathrm{L}$ of $0.4 \%$ trypan blue. After mixing, the $10 \mathrm{uL}$ of cell suspension was loaded onto haemocytometer. The viable (colorless) and dead (blue) cells were counted in each large square of the haemocytometer using a 40x objective for both untreated (control) and sodium-arsenite-treated (stress) cells.

\subsubsection{Animal time course and sample collection}

All animal experiments were conducted according to the ethical standards of the Regierungspräsidium Tübingen (Regional Council) experimental no. FLI 231/07 file reference number 35/9185.81-2. All procedures were carried out in accordance with the institutional and French national guidelines within the European Community Council Directive 86/609/EEC. The experimental procedures approved by the INRA Toulouse/ENVT ethics committee were used. 
The mice model ( $3 x \mathrm{Tg}-\mathrm{AD}$ ) was initially generated and characterized by Oddo et al. (2003). Transgenic mice between 3-4 months of age were inoculated with $10 \%$ brain homogenates in PBS from AD-patients, in the thalamus. Both, inoculated and noninoculated control animals were sacrificed at 4 time-points corresponding to early pre-symptomatic (3 months post-inoculation [mpi]), late pre-symptomatic (6 $\mathrm{mpi}$ ), early symptomatic (9 mpi) and late symptomatic (12 mpi) stages of the disease, and the brains were collected. All mice $(n=48)$ were anesthetized and decapitated. The cortical tissues were obtained and immediately stored in liquid nitrogen for further analysis. A graphical representation of the mice model indicating different time points of the sample collection is given in Fig. 8 .

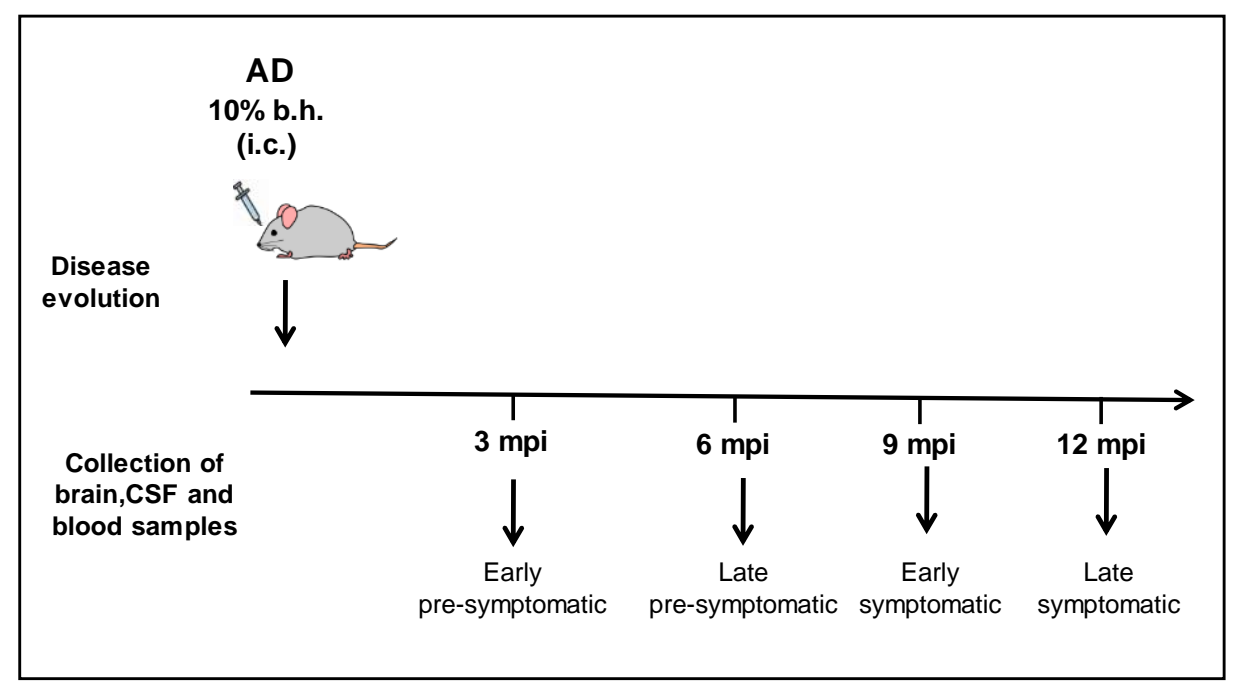

Figure 8: Graphical representation of animal experimentation using the mice model of Alzheimer's disease. Transgenic mice model of $A D(3 \times T g-A D)$ was used for inoculation. Brain homogenates (b.h.) (10\%) from AD patients were injected into thalamus. The samples were collected for early pre-symptomatic ( 3 months postinoculation [mpi]), late pre-symptomatic (6 mpi), early symptomatic (9 mpi) and late symptomatic (12 mpi) stages of the disease.

\subsubsection{Statistical analysis}

All the data in the present study was obtained after performing at least three independent experiments. All the results are described as mean \pm SEM (standard error of the mean). The densitometry analysis of immunoblots was performed with Image Lab software (version 3.0.1). Statistical tests were applied using GraphPad Prism 6.01. The data from mass spectrometry analysis were analysed using the Perseus software with adjustments for multiple testing. Hierarchical clustering analysis was also performed with Perseus software. Two-dimensional interaction plots were plot- 
ted in R (version 3.4.3), followed by editing using Inkscape (version 0.92). For comparisons between two groups, the Student's $t$-test or Welch's $t$-test was used. For comparisons between three or more groups, a one-way ANOVA followed by the Tukey post-hoc analysis was used. Statistical significance was considered for a $p$ value $<0.05$ 


\section{Results}

Emerging evidence has demonstrated that numerous neurodegenerative disorders including AD display features of RBP pathologies, highlighting a vital role of RBPs in neurodegeneration (Cookson, 2017; Vanderweyde et al., 2016). The study of RBP biology is at its early stages, particularly in Alzheimer's and prion diseases. To this end, we identified and characterized the RNA-binding proteome variations in Alzheimer's and prion diseases.

This chapter is divided into four major sections. The first section describes the identification and characterization of RNA-binding proteomes from postmortem brains of diseased and healthy subjects. Target candidates were prioritized from proteomic analyses for further investigation. The second section is focussed on pathological characterization of target RBPs, particularly SFPQ, in the human brain. The third section describes the cellular model of stress and the tau-pathology model, and, in addition, explores the mechanistic link of SFPQ in disease pathogenesis and progression. This chapter links SFPQ in the human brain to stress granules and tau protein. Finally, in the fourth section, SFPQ and associated proteomic signatures are studied at pre-symptomatic and symptomatic stages of the disease in the 3xTg-AD mice model, in order to uncover early changes during the disease progression (Fig. 9).

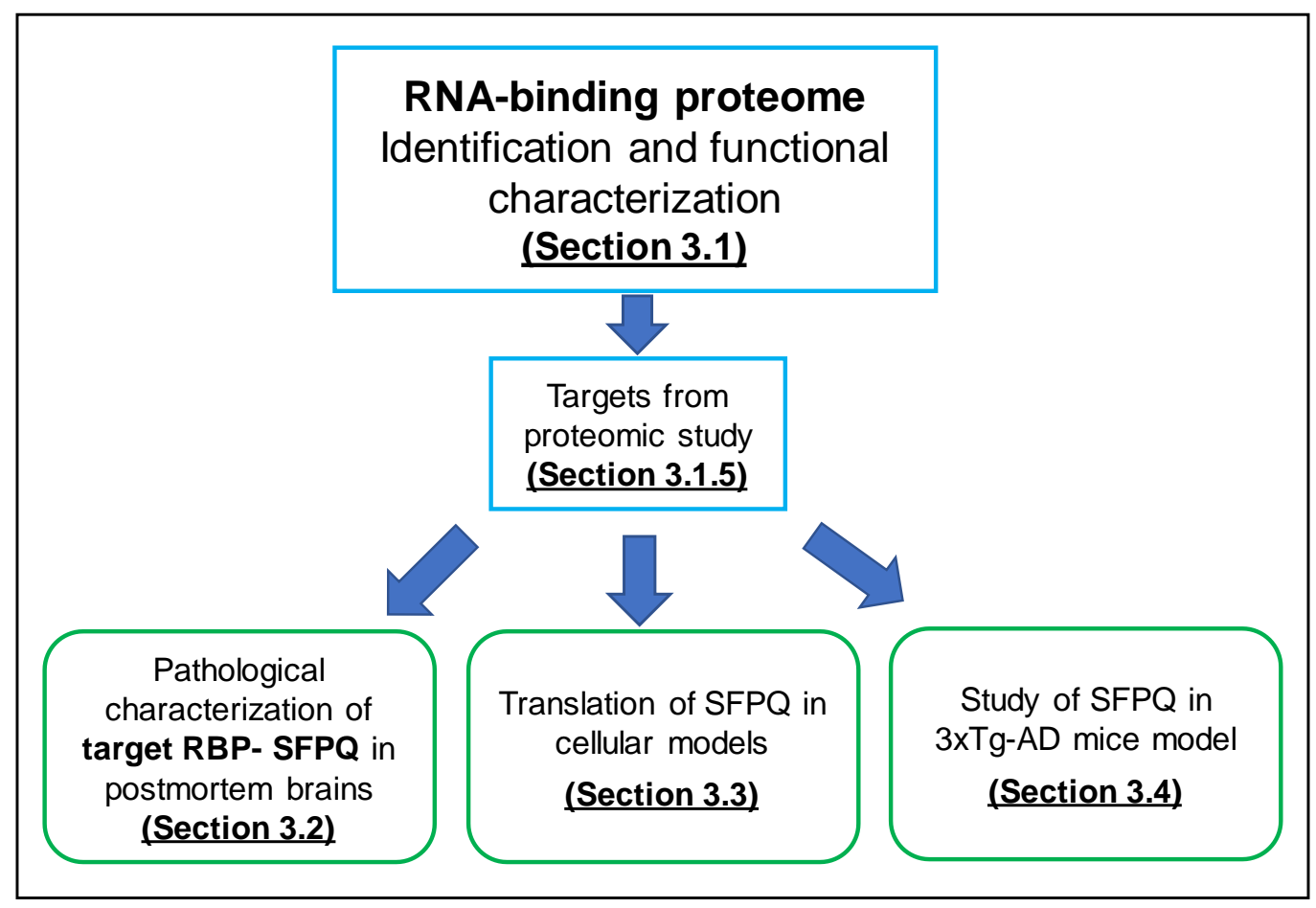


Figure 9: Overview of the results: In the first part of the study, RNA-binding proteins were identified and characterized in postmortem brains from diseased and healthy subjects (Section 3.1). Targets were prioritized for further investigation from the proteomic study (Section 3.1.5). One of these target RBPs, the RNA-binding protein SFPQ (splicing factor proline and glutamine rich), was pathologically characterized in the human brain (Section 3.2). Then, these target candidates were translated in the cellular (Section 3.3) and animal models (Section 3.4) to investigate mechanistic links with pathological features of the disease.

\subsection{RNA pull-down assay and mass spectrometry analysis of RNA- binding proteome}

In this study, we utilized brain-derived, total RNA to pull down RBPome complexes from the human brain of spAD, rpAD, sCJD as well as control subjects. Mass spectrometry analysis was performed to identify and quantify isolated RNA-binding protein complexes. A schematic outline of the RNA pull-down/MS procedure used in this study is shown in Fig. 10A.

Total RNA was isolated from the frontal cortical region of the brain of diseased and control subjects. Brain-isolated RNA was labelled with desthiobiotin at 3'-end to minimize any disturbance to the secondary structure of the RNA. Desthio-biotinylatedRNA was bound to streptavidin magnetic beads and oriented to enrich RNA-binding protein complexes. Isolated protein complexes were identified by two MS approaches: global proteomic approach (spectral counting-based, label-free quantification [LFQ]) and a variant of data-independent quantitative MS (Sequential Window Acquisition of All Theoretical Mass Spectra [SWATH-MS]) (Fig. 10A).

After the identification and quantification of RBPome candidates, a combination of bioinformatic, computational, and biochemical approaches was used to comprehensively analyse the isolated RBPome (Fig. 10B). 


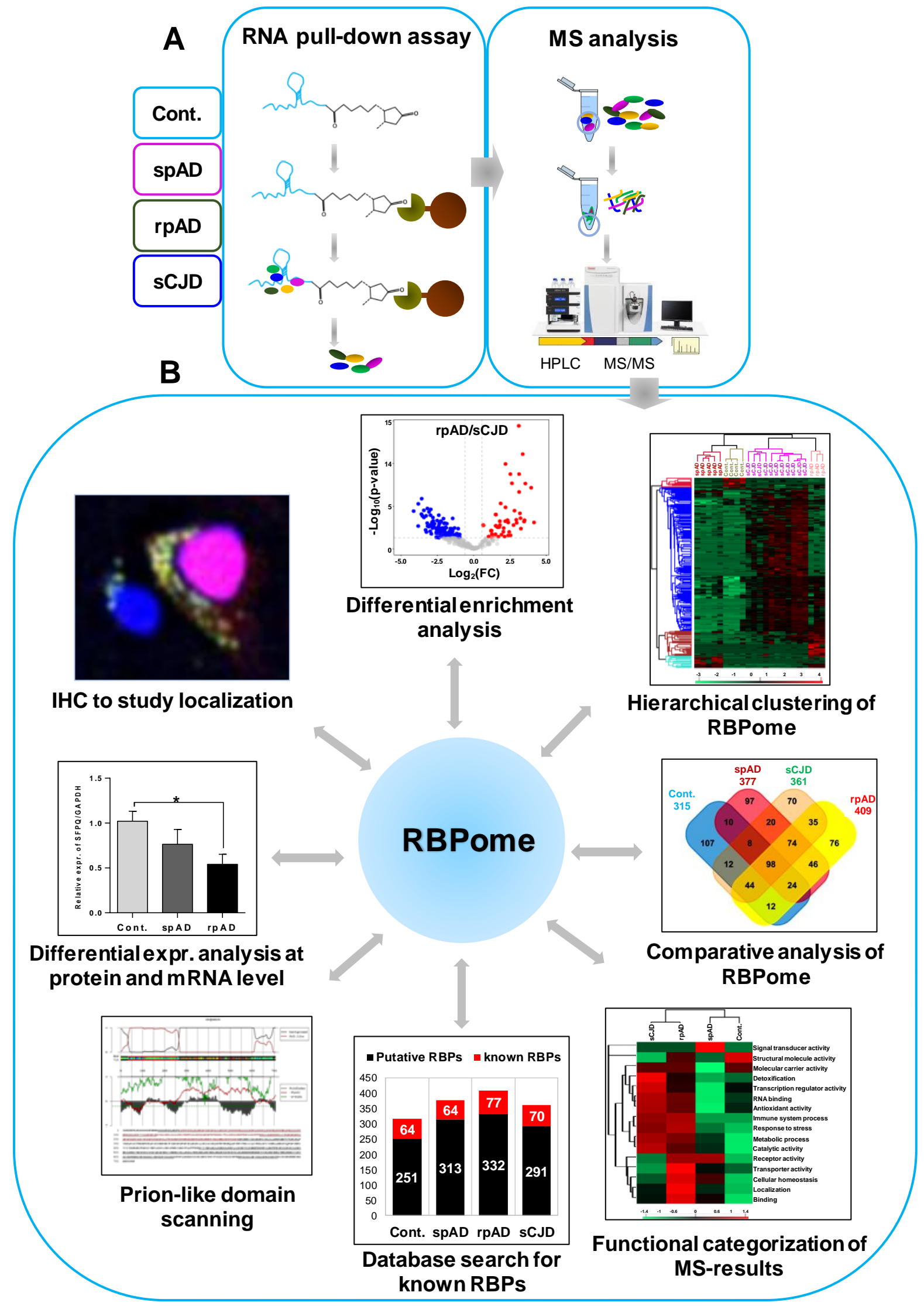

Figure 10: Identification of RNA-binding proteome by total RNA pull-down assay coupled with mass spectrometry analysis. A) Total RNA was isolated from the frontal cortical region of 20 diseased and control cases. Brain-derived RNA was desthiobiotinylated at 3'-end to minimize disturbance to the secondary structure of 
RNA. Desthiobiotinylated RNA was bound with streptavidin magnetic beads. Bead-bound RNA was oriented to pull down RBP complexes from total tissue lysates of corresponding diseased and healthy subjects. Bead-only controls were used for non-specific binding. Isolated RBP complexes were digested into peptides followed by identification and quantification by mass spectrometry. In total, 1,091 proteins were detected and quantified at a minimum peptide count of 2 and a protein confidence threshold of $99 \%$. B) Target selection from proteomic investigation and their pathological characterization in the postmortem brains. A combination of bioinformatic and computational approaches was used to identify significant hits from the proteomic study, including differential enrichment analysis of MS data, hierarchical clustering analysis to visualize global proteome profile, comparative RBPome analysis for similar and unique proteins, Gene Ontology (GO) functional enrichment analysis, database search for identification of bona-fide and novel or putative RBP candidates, and prion-like domain scanning with the PLAAC database. Target candidates from proteomic investigation were pathologically characterized in the postmortem human brain, using various techniques including immunoblotting, qRT-PCR, and immunohistochemical analysis.

\subsubsection{Global enrichment profile of RNA-binding proteome}

Label-free quantitative mass spectrometry analysis identified a total of 1,091 proteins, with minimum peptide count of 2 and a protein confidence threshold of $99 \%$. For detailed analysis of the identified proteome, three strategies were adopted (Fig. 11). A detailed description of all the approaches (A, B and $C$ ) can be found in "Methods Section". In approach A and B, two subtypes of SCJD (MM1 and VV2) were merged, and only proteins that were common in both subtypes were considered to be a general representation of the SCJD group. Firstly, to gain insight into the global similarities and differences between disease groups, RBP-candidates were uploaded to Perseus software, followed by pairwise comparisons between all the group combinations using $t$-test (approach A). The proteins having a p-value $<0.05$ and FC $>$ \pm 1.5 were considered as significantly abundant between any of the experimental groups (Fig. 12A).

Using significantly enriched proteins in the hierarchical clustering analysis, four different expression signatures were found, which are represented in the form of heatmap (Fig. 12B). Columns of the heatmap represent all the disease groups, while the rows represent significantly abundant proteins. Clustering of the biological replicates across the vertical axis generated two main column clusters. Based on RBP signatures, rpAD and SCJD groups appeared largely similar as they were segregated into a single cluster and differed from the control group (Fig. 12B). The control and spAD cases were segregated into a separate cluster based on the full set of differen- 
tially enriched proteins, which indicated similarities in the protein profiles between control and spAD groups.

Overall, clustering of the individual replicates indicated low biological variance and segregation of all the groups, showing the reproducibility and robustness of the workflow (Fig. 12B).

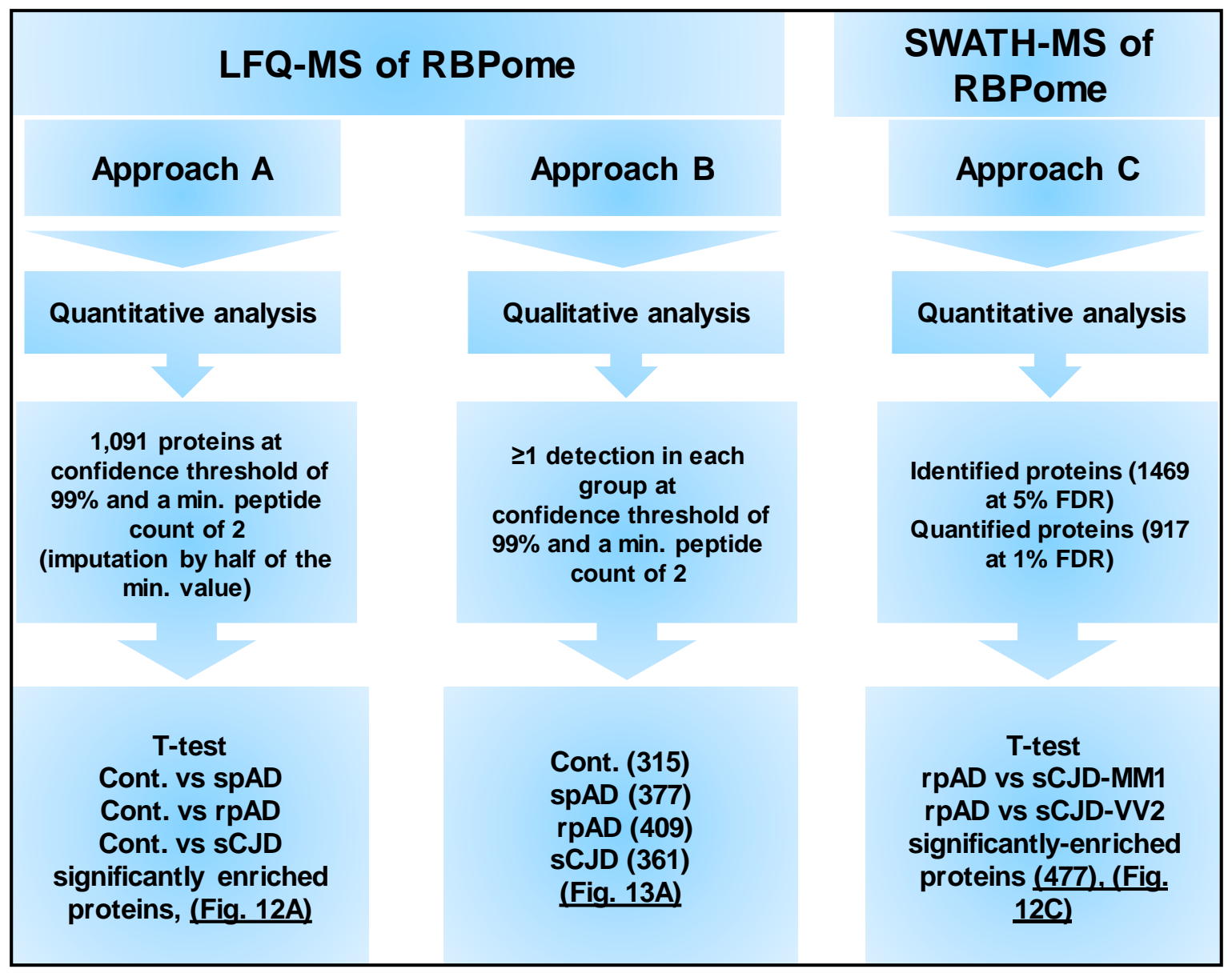

Figure 11: Statistical analysis of RBPome candidates: Two different strategies (approaches A and B) were used to obtain a more detailed analysis of RBPome candidates identified from LFQ-MS. Approach A: Statistical analysis of isolated proteomic candidates by Perseus software. Zero values from the total spectral counts of the proteins were imputed by half of the minimum value followed by pairwise Student's $t$-test comparisons between all the group combinations, to find out significantly enriched proteins in each group. Approach B: Qualitative analysis of the RBPome. Proteins with a single quantitative value were included in this approach from all the groups to have a broader impression of the isolated proteome. In order to find out common and unique proteins among all the groups, a comparative RNA-binding proteome profile was obtained, based on specifically identified proteins in each group. Approach C: SWATH-MS of rpAD and SCJD (MM1 and VV2 subtypes) followed by pairwise comparisons, as above. The numbers of resulted proteins, and selected proteins is described in figures as indicated. 
Given the exploratory nature of this discovery-phase proteomics workflow, we relaxed the criteria and included all the proteins with one or more than a singledetection in each group, due to the high sensitivity of the MS analyser (approach B) (Fig. 11). The proteins which were specifically enriched in each group were further analysed to have a comparative proteome profile of the RBP-candidates. This analysis revealed disease-subtype specific differences in the enriched RBP candidates (Fig. 13A). There were 98 proteins that were shared among all the groups. The common and unique proteins from all the groups are shown in figure (Fig. 13A, annexure data Table 15).

The RBPome variations were highly prominent in the SCJD and rpAD groups, as evidenced by the global enrichment profile of both groups (Fig. 12B). We used a variant of data-independent quantitative MS, namely SWATH-MS, to gain a deeper insight into rpAD and SCJD RBPome candidates (Fig. 11, approach C). The SWATH-MS variant has emerged as a technology that combines the deeper proteome coverage with the quantification accuracy.

This analysis identified 1469 proteins at FDR of 5\%, while 917 proteins were quantified at a critical FDR of $1 \%$. Bioinformatic analysis (Welch's $t$-test with BenjaminiHochburg correction) indicated 477 proteins that were differentially modulated in abundance between IPAD and the two subtypes of SCJD, MM1 and VV2. One interesting finding, evident from the hierarchical clustering analysis of the significantly modulated RBP signatures, was the similarity in the RBPome profile between rpAD and the SCJD-subtype MM1, when compared to the SCJD-VV2 subtype. Both groups (rpAD and SCJD-MM1) were segregated into a single cluster, whereas the SCJDVV2 subtype was segregated into a separate cluster (Fig. 12C). 
A
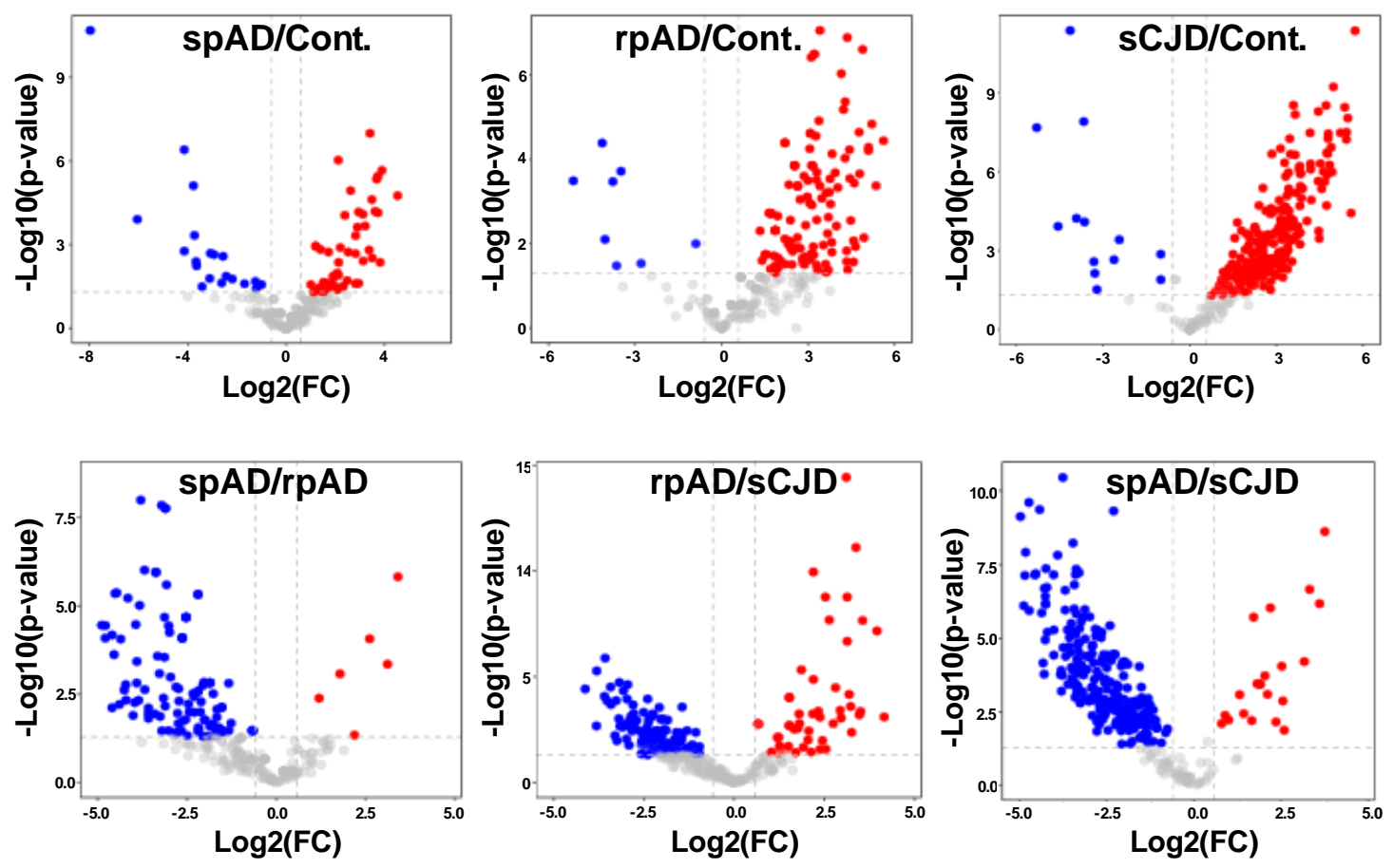

B

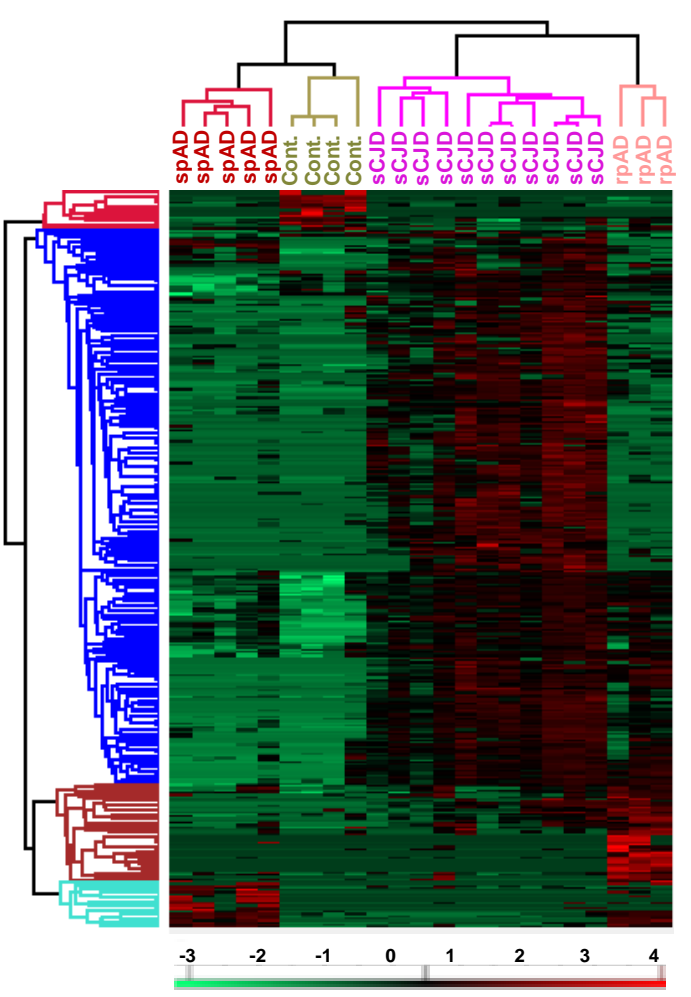

C
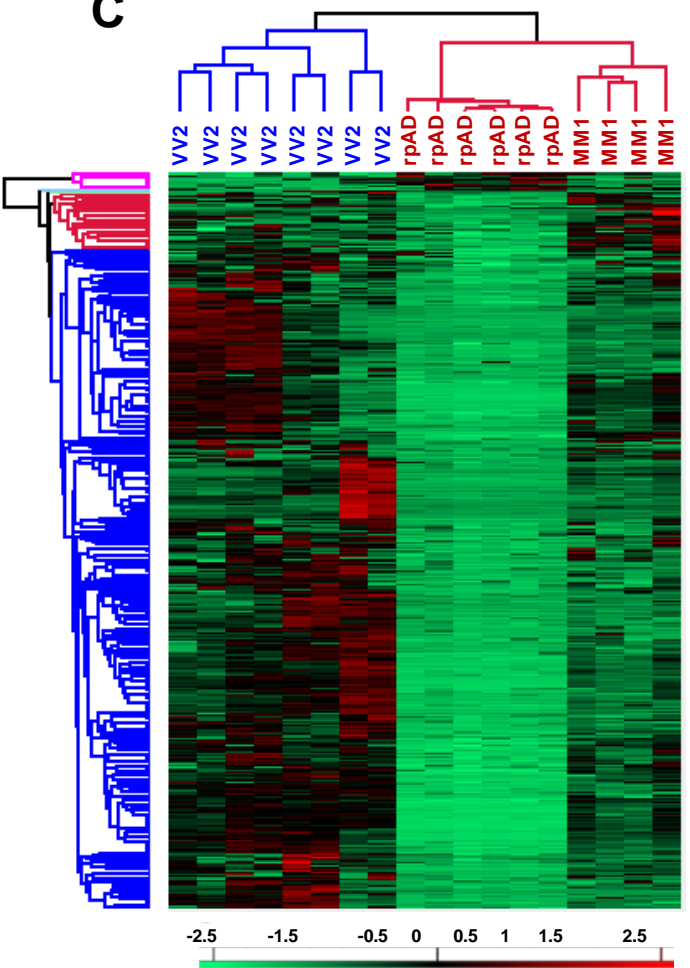

Figure 12: Global enrichment profile of RBPome candidates: A) Volcano plots of pairwise comparisons displaying, the - $\log _{10}-p$-values ( $y$-axis) and the $\log _{2}(F C)$ of the proteins that were significantly abundant ( $x$-axis) in all the group combinations ( $s p A D$ vs control, rpAD vs control, sCJD vs control, spAD vs rpAD, rpAD vs sCJD, and spAD vs sCJD). The dashed lines indicate Student's $t$-test cut-off, the data points above the dashed lines represent proteins having a $p$-value $<0.05$ and $F C> \pm 1.5$ as significant hits, which are depicted in red (for enriched) and blue (for depleted). B) The heatmap of hierarchical clustering of significantly enriched proteins. The $\log _{2}-$ 
transformed expression values were normalized by Z-score for each biological replicate. Horizontal axis indicates the differentially enriched proteins, and the vertical axis shows the biological replicates from all the groups. Green denotes depleted proteins, red represents enriched proteins. C) The heatmap of hierarchical clustering from SWATH-MS of rpAD and SCJD-MM1 and -VV2 subtypes, showing similarities in RBPome candidates between rpAD and SCJD-MM1 subtype.

\subsubsection{Functional categorization of RBP candidates}

To gain functional insights into the identified RBP candidates, classification based on Gene Ontology (GO) annotations was performed (Fig. 13B). The analysis was performed in the GO domains, including "Biological Process and Molecular Function". The results of this analysis identified several categories that were differentially enriched in various disease groups.

To compare the functional profiles and to visualize the relative enrichment of different categories in all the groups (Fig. 13B), the protein counts belonging to GO terms from each group were uploaded to Perseus software. The variation in each term across the groups was calculated by a Z-score. The heatmap is representing disease groups across the columns (vertical axis) and functional terms in the rows ( $\mathrm{x}$ axis). The enriched terms in comparison to other groups are indicated by red, while depleted terms are indicated by green.

A large number of identified proteins were related to cellular and metabolic processes, RNA metabolism, and immune and stress responses, among other activities. Consistent with the analysis of the RNA-binding proteome, many proteins were annotated as having nucleic acid, nucleotide, RNA- and/or DNA-binding activity as well as transcriptional regulatory and transporter activities (Fig. 13B). A variety of proteins were annotated as having catalytic activity (including lipid and carbohydrate metabolic enzymes and protein modulating enzymes), structural molecule and signal transducer activity (Fig. 13B).

Based on clustering of GO terms, rpAD and SCJD groups were segregated into a single cluster, showing similarities in the enriched functional terms (Fig. 13B). The most enriched processes for both groups included response to stress (e.g. response to oxidative stress and cellular response to stress), metabolic process and catalytic activity, among others (Fig. 13B). The signal transducer activity was particularly en- 
riched in the SpAD group when compared with other groups. Additionally, one cluster of cellular processes, including cellular homeostasis, localization and binding, was particularly enriched in the rpAD group, showing more aggressive disturbances in the overall cellular homeostasis of the rpAD group. Taken together, these results suggested that the RBPome aberrations are an integral part of the pathological features of particularly the rapidly progressive forms of dementia. 


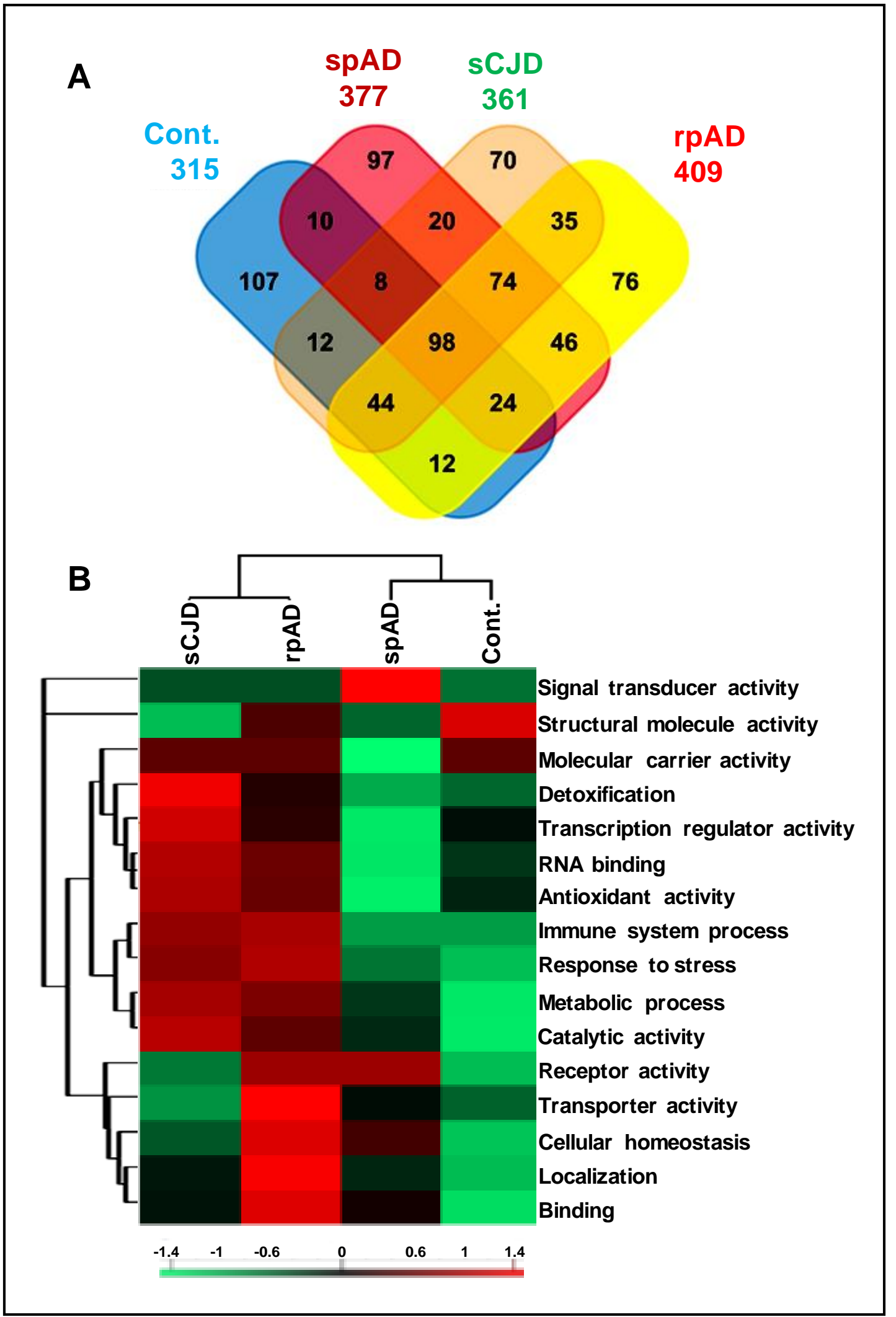

Figure 13: Comparative RNA-binding proteome profiling and functional categorization of MS results. A) Venn diagram showing similar and unique RBP candidates in each group. There were ninety-eight proteins common among all the groups. There were total of 315 proteins in the control, 377 in the spAD, 361 in the sCJD and 409 in the rpAD group. Of these, 107 were only uniquely present in the control, 97 were only found in the spAD, 70 in the sCJD and 76 in the rpAD group. B) Functional categorization of MS results: RBP candidates 
identified in each group were analysed for associated GO terms. The protein count associated with the GO terms from each group was uploaded to Perseus software, to prepare heatmap showing relative enrichment of different functional categories across all the groups. The variation in each term across the groups was calculated by Zscore. The heatmap is representing disease groups across the columns (vertical axis) and functional terms in the rows ( $\mathrm{x}$-axis), with red indicating an enriched category as compared with other groups, and green indicating depleted terms.

\subsubsection{Classification of known and putative/novel RBP candidates}

Candidates for RNA-binding protein were classified as previously demonstrated (Beckmann et al., 2015). In order to discriminate bona-fide RBPs from putative RBP candidates, identified proteins were searched for RNA-binding annotation in the UniProtKB database. Two categories were organized to give clarity to the data, as described previously (Zhang et al., 2016) (Fig. 14A). The category I (bona-fide RBP candidates: red bars) contains proteins that were annotated as RNA-binding in the UniProtKB database. There were 64 RBPs in the control group, 64 in spAD, 77 in rpAD and 70 in SCJD that were annotated as RNA-binding proteins (Fig. 14A). The category II (putative/novel RBP candidates: black bars) contains the rest of the proteins that have neither a known RNA-binding domain (RBDs) nor a known linkage to the RNA. The putative RBP candidates include metabolic enzymes and translation factors among others. It should be noted that some of the identified candidate proteins may not have RNA-binding activity themselves, but, instead, interact with other proteins that do, and are hence identified in the RBPome.

\subsubsection{Prion-like domain (PLD) prediction}

Prion-like domains are often present in RBPs, which carry out protein aggregation in neurodegenerative diseases, e.g. amyotrophic lateral sclerosis (ALS), and, more recently, in AD (King et al., 2012; Wolozin, 2012; Vanderweyde et al., 2016). To evaluate the presence and functional implication of PLD-containing proteins, the identified RNA-binding proteomic candidates were analysed using highly stringent computational algorithms (Batlle et al., 2017; Michelitsch and Weissman, 2000; Toombs et al., 2012; Zambrano et al., 2015). In this study, the web-based PLAAC database was used to identify domains with prion-like amino acid sequences. The input sequences are ranked by several types of summary scores. Criteria for a protein to have a PLD consisted of four requirements: it had to 1) be rich in $\mathrm{Q} / \mathrm{N}$ - 
sequences, 2) exhibit disorderliness according to the PAPA algorithm (Prilusky et al., 2005), 3) have compositional similarity with yeast prion domains, and 4) contain short amyloidogenic stretches able to carry out their self-assembly into an amyloidogenic state. Applying these criteria, we identified twenty-four PLD-containing proteins in the current study (Table 10).

Table 10: Prion-like domain prediction score of RBPs from PLAAC database.

\begin{tabular}{|c|c|c|c|}
\hline Experimental group & SEQid & COREscore & PAPAprop \\
\hline Cont., sCJD & sp|Q12906|ILF3 & 30.688 & 0.092 \\
\hline Cont. rpAD, sCJD & sp|P08247|SYPH & 25.009 & -0.105 \\
\hline Cont. SpAD, sCJD & sp|Q5D862|FILA2 & 24.633 & 0.109 \\
\hline Cont., spAD, rpAD, sCJD & sp|P17600|SYN1 & 20.311 & -0.054 \\
\hline Cont, sCJD & sp|O43390|HNRPR & 17.059 & -0.049 \\
\hline Cont., sCJD & sp|P09012|SNRPA & 11.165 & -0.146 \\
\hline spAD, rpAD & sp|Q9UPA5|BSN & 22.864 & -0.044 \\
\hline spAD, rpAD, sCJD & sp|P20073|ANXA7 & 17.868 & -0.033 \\
\hline spAD, sCJD & sp|Q92945|FUBP2 & 15.926 & -0.065 \\
\hline spAD, rpAD, sCJD & sp|P02671|FIBA & 12.12 & 0.033 \\
\hline rpAD, sCJD & sp|Q14103|HNRPD & 30.124 & 0.164 \\
\hline rpAD, sCJD & sp|P23246|SFPQ & 28.671 & -0.1 \\
\hline rpAD & sp|P04156|PRIO & 14.844 & 0.02 \\
\hline SCJD & sp|Q92734|TFG & 38.015 & -0.005 \\
\hline SCJD & sp|Q01844|EWS & 34.368 & 0.057 \\
\hline SCJD & sp|P22626|ROA2 & 30.362 & 0.043 \\
\hline SCJD & sp|P09651|ROA1 & 28.381 & 0.093 \\
\hline SCJD & sp|Q8WUM4|PDC6 & 26.882 & 0.049 \\
\hline SCJD & sp|P50995|ANX11 & 19.803 & -0.059 \\
\hline SCJD & sp|Q9UBV8|PEF1 & 19.787 & 0.005 \\
\hline SCJD & sp|P17931|LEG3 & 17.868 & -0.033 \\
\hline sCJD & sp|P49840|GSK3A & 11.251 & -0.103 \\
\hline SCJD & sp|P14678|RSMB & 10.958 & -0.121 \\
\hline sCJD & sp|O60506|HNRPQ & 8.799 & -0.019 \\
\hline
\end{tabular}

PLAAC: Prion-like amino acid composition, SEQid: sequence ID from fasta file, COREscore: max sum of PLAAC LLRs, PAPAprop: max score of PAPA prion propensities (Toombs et al., 2012), Cont.: control, spAD: sporadic Alzheimer's disease, rpAD: rapidly progressive Alzheimer's disease, sCJD: sporadic Creutzfeldt-Jakob disease.

Among all the identified PLD-containing proteins, splicing factor proline and glutamine rich (SFPQ) with a PLD score of 28.67 was an interesting target as this protein was specifically enriched in rapid forms of dementia ( $r p A D$ and SCJD). The detailed architecture of the SFPQ prion-like domain is described in the figure (Fig. 14B). 
A

- Putative RBPs a known RBPs

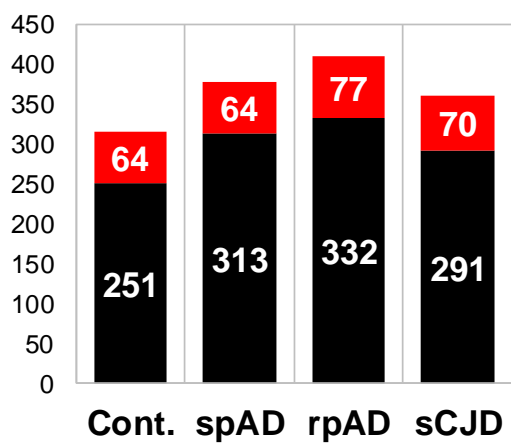

B

N Q Y G M P A H T F R V I D L K C W E

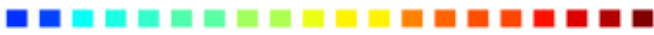

Sequence

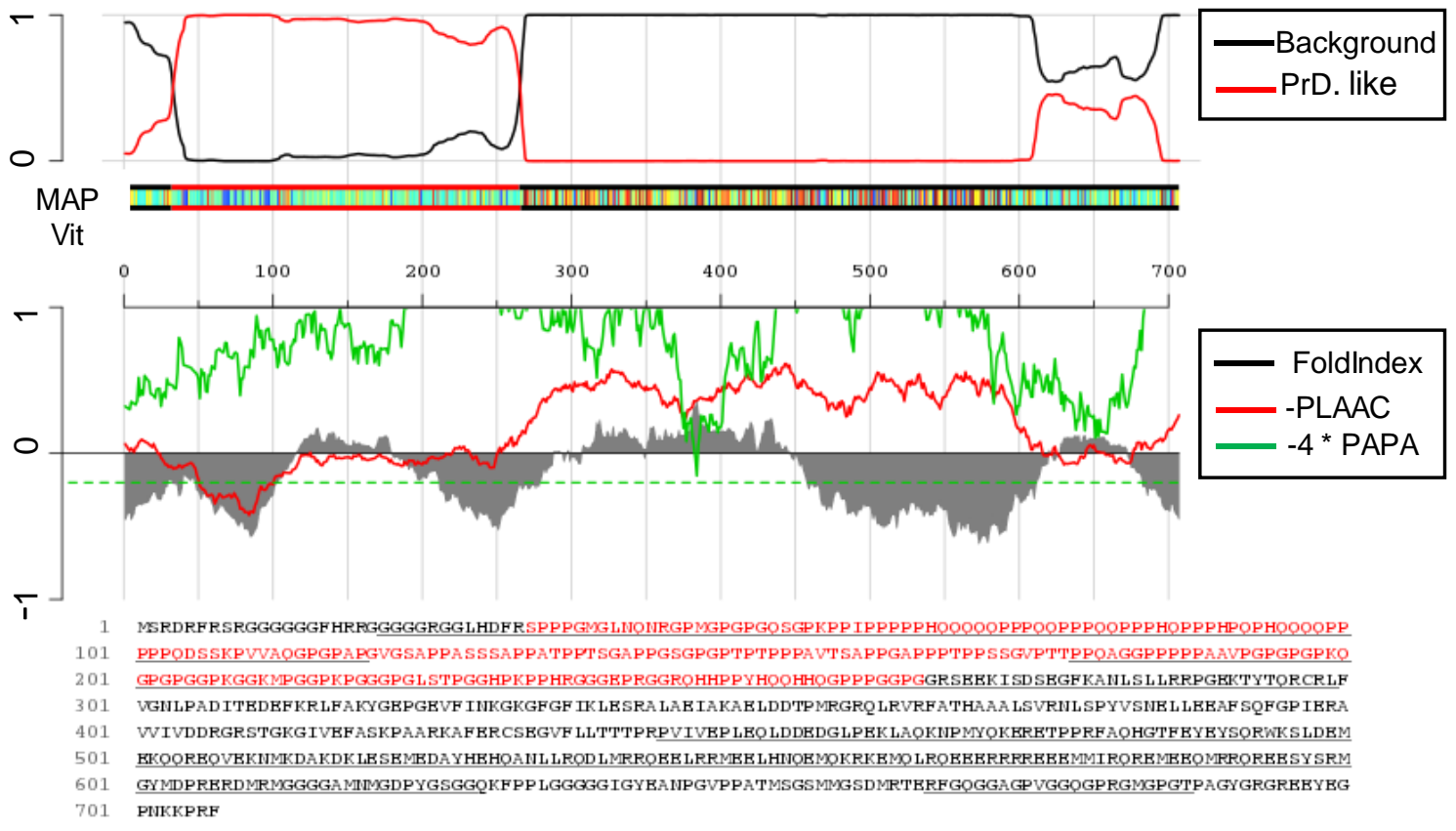

Figure 14: Identification of bona-fide and putative RBP candidates. A) The proteins identified in the proteomic study were searched for RNA-binding annotation in the UniProtKB database. The bar graph shows two categories of identified protein candidates. The category I (red bar) indicates bona-fide RBPs (known), and category II (black bar) represents potential novel/putative RBP candidates from each group. B) Identification of SFPQ-prionlike domain by PLAAC database. The amino acid composition of SFPQ is represented in colour-coded boxes. The red line in the top panel represents the probability of having a prion domain against the background. The plots in the middle panel show fold-index scores in grey (Prilusky et. al., 2005), the log-likelihood (LLR) ratio scores in red (Alberti et. al., 2009), and the predicted prion propensity (PPP) in green (Toombs et. al., 2010). Negative scores represent disorder and prion propensity, dashed green line is indicating the cutoff value of PPP $>0.05$. The bottom panel shows the primary sequence of SFPQ with the PLD in red (Alberti et. al., 2009). 


\subsubsection{Target candidates from proteomic study}

From the proteins which had prion-like domains (as described in the Table 10), the SFPQ was selected (approach B). This protein was specifically identified in the rpAD and SCJD groups and exhibited a high score for PLD. This high score of PLD is a crucial factor contributing to the pathophysiology of RBPs. Given the involvement of stress granules in the $A D$, valosin-containing protein (VCP) was selected from results using the approach $\mathrm{A}$. The valosin-containing protein plays an important role in the formation and clearance of SGs. Valosin-containing protein was also specifically enriched in rapid forms of dementia ( $r p A D$ and $S C J D)$.

One interesting finding evident from the proteomic study was the enrichment of the microtubule-associated protein tau (MAPT), a major hallmark of AD in our RBPome dataset, suggesting an intimate link of RBPs to tau-related pathological mechanisms. The interactive association between tau, SFPQ and VCP led us to investigate the role of these target candidates in the disease. Furthermore, TIA-1 (T-cell intracellular antigen 1) was also added to the candidate list for further investigation (Fig. 15), given that its role in tau aberrations in AD (Apicco et al., 2018), although it was not detected in our MS dataset, most likely due to the hydrophobic nature of the protein.

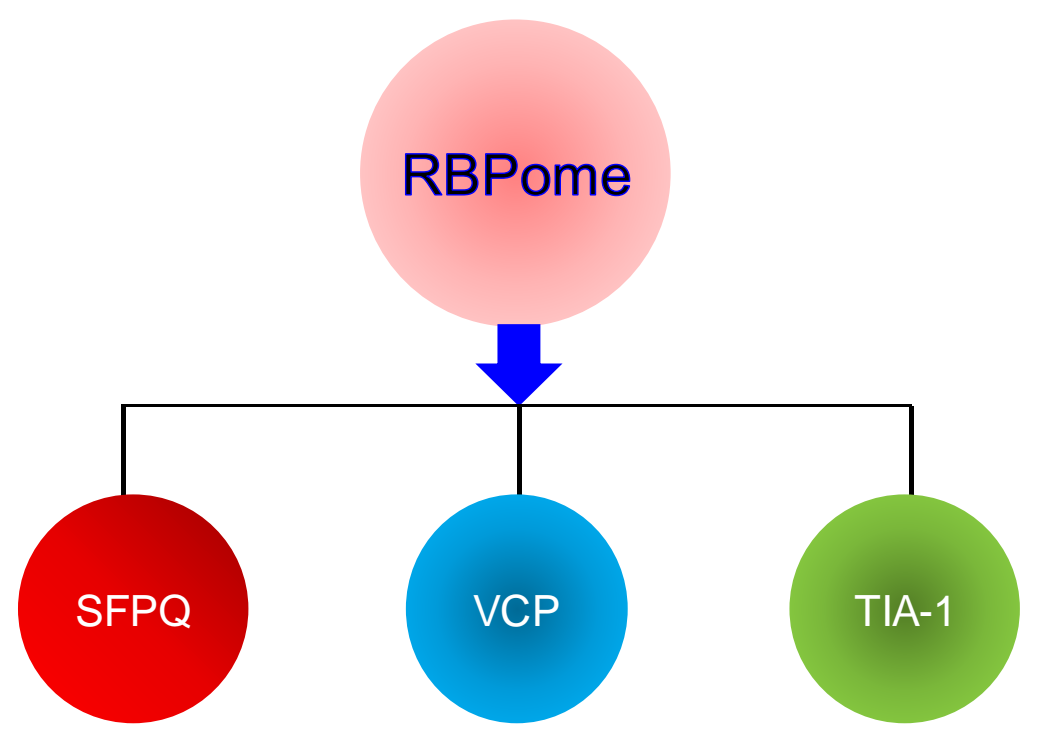

Figure 15: Candidate hits from proteomic investigation. Splicing factor proline and glutamine rich (SFPQ) (approach B) was selected, because the protein was specifically enriched in rpAD and SCJD, and, in addition, bearing high score for PLD; which is very crucial factor contributing to pathophysiology of RNA-binding proteins. Given the recent role of stress granules in $A D$, valosin-containing protein was selected (approach $A$ ). This protein was significantly enriched in abundance only in diseased groups and has a role in the formation and clearance of SGs. Due to involvement of TIA-1 protein in the pathophysiology of tau, it was also added to the target candidate list, although it was not detected in our dataset. 
In order to more accurately illuminate the specific mechanisms of how selected targets (particularly SFPQ) interact with other major cellular components (e.g. tau) in the development of $A D$, and to further ascertain whether the aforementioned selected approaches were appropriate for the target selection, we began to explore these proteins more in-depth. Based on research which has shown that neurons are particularly vulnerable to aberrant dosage as well as the dynamics of RBPs (Conlon and Manley, 2017), the first set of analyses focused on the pathological characterization of these target proteins in the postmortem brains, using various techniques including immunoblotting, immunohistochemistry, and qRT-PCR analysis. Then, these target candidates were translated in the cellular and animal models to find out mechanistic links with pathological features of the disease.

\subsection{Pathological characterization of target RBP (SFPQ) in the post- mortem human brain}

\subsubsection{SFPQ is dysregulated in rPAD and SCJD brains}

To investigate the differential expression of SFPQ in Alzheimer's and prion diseases, immunoblotting analysis was performed in the frontal cortical brain tissues of human brains from the spAD, rpAD, sCJD-MM1, and sCJD-VV2 subtypes as well as nondemented controls. Information on all the subjects is listed in annexure data tables (Tables 11 and 12). Immunoblotting analysis showed that the expression of SFPQ was reduced in the frontal cortex of both rpAD and SCJD patients compared with controls (Fig. 16A).

For SFPQ expression in spAD patients, a decreasing trend was observed, though that was not significant. The band intensities of SFPQ were normalized to the corresponding levels of GAPDH by calculating the intensity ratio of the GAPDH bands. The densitometric analysis revealed that SFPQ levels were remarkably decreased in both subtypes of SCJD (MM1 and VV2) compared with the control subjects (Fig. 16B).

A significant reduction was observed for TIA-1 levels in SpAD and both subtypes of SCJD (MM1 and VV2), when compared with control subjects (Fig. 16A and C). In contrast, the levels of TIA-1 were significantly increased in rpAD patients in comparison to patients in the spAD group. Defects in VCP have been linked with aberrant 
dynamics of SGs, both as impaired formation and clearance of SGs (Buchan et al., 2013). Next, we sought to examine the levels of VCP in the diseased and control subjects. An increasing trend was observed for VCP levels in rpAD and SCJD-VV2 subtype (Fig. 16D) compared with the respective controls, suggesting an impaired SG dynamic.

Overall, the dysregulated target proteomic signatures suggest a compromised multifunctionality of these proteins, which can start a cascade of aberrant signaling in the neurons and contribute to neurodegeneration. 
A

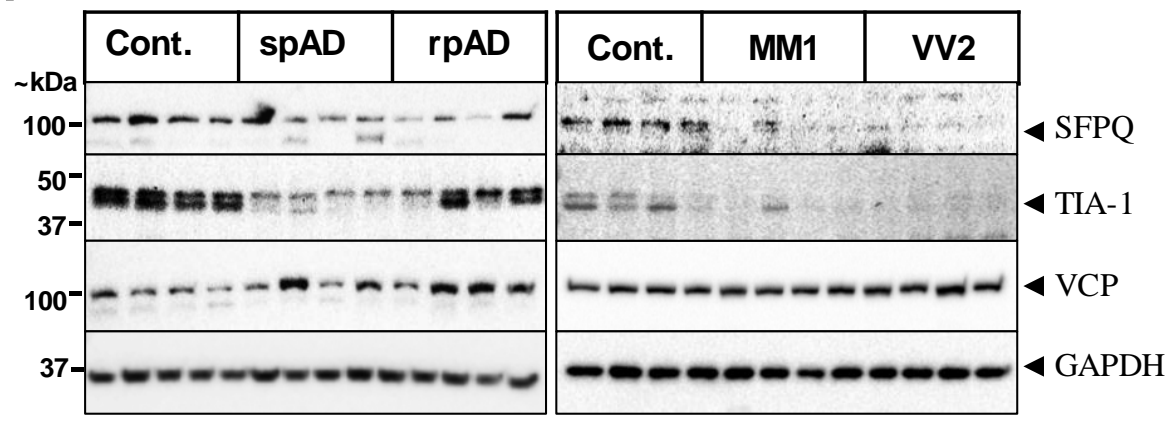

B

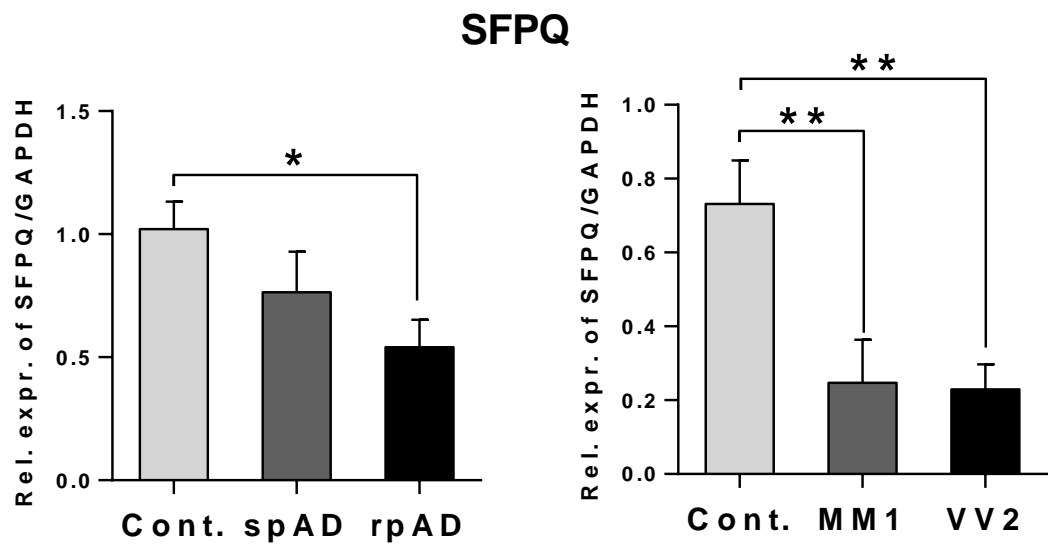

C

TIA-1
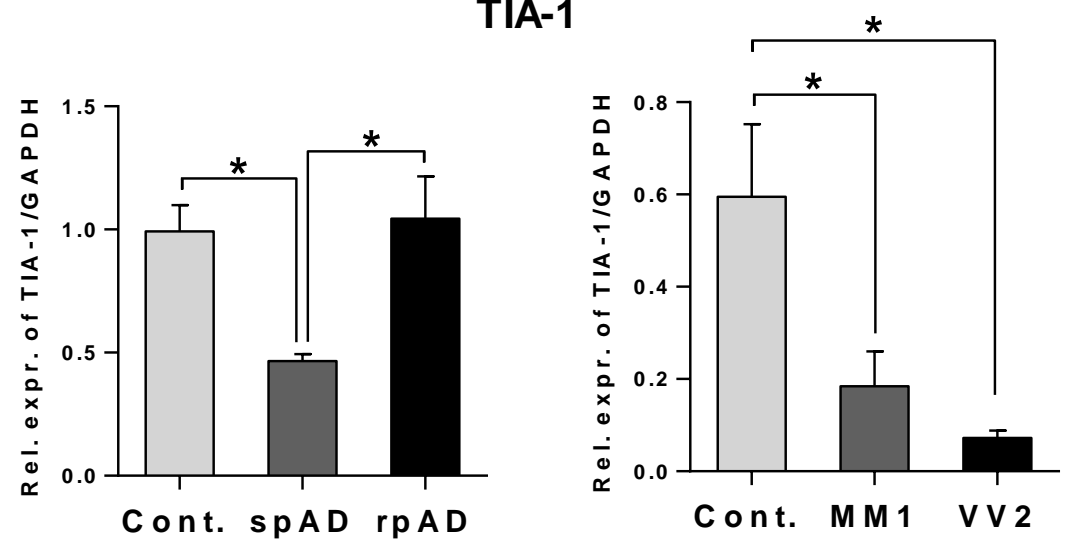

D

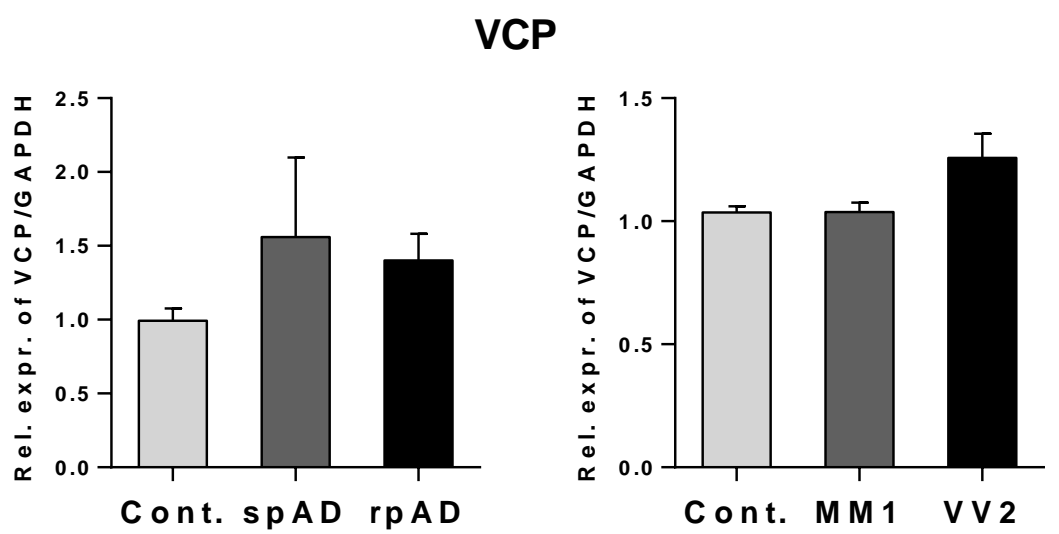

Figure 16: Differential expression analysis of SFPQ, TIA-1, and VCP: A) Immunoblotting analysis was performed with frontal cortical human brain tissues from $\operatorname{spAD}(n=7), \operatorname{rpAD}(n=7)$, sCJD (MM1 and VV2 subtypes, 
$\mathrm{n}=8)$ and non-demented controls $(\mathrm{n}=8)$. Representative immunoblot images are shown. B-D) The densitometric analysis of SFPQ, TIA-1 and VCP. Protein amounts were normalized to corresponding bands of GAPDH. The levels of SFPQ were significantly reduced in rPAD and the indicated SCJD subtypes. Expression of TIA-1 at the protein level was significantly reduced in the SPAD and SCJD groups, while it was increased in rpAD as compared with the spAD group. For VCP, no significant changes were observed. One-way ANOVA was conducted, followed by Tukey post-hoc test for multiple comparisons, ${ }^{*} p<0.05$, ${ }^{* *} p<0.01$.

Dysregulated protein levels of SFPQ and TIA-1 prompted us to investigate the mRNA levels of these target proteins in the diseased brains. Quantitative RT-PCR was used to examine mRNA levels in SPAD, rpAD and control subjects. The expression levels were normalized to GAPDH. Contrary to a reduced expression at the protein level in rpAD, mRNA levels of SFPQ were significantly elevated in the rpAD group compared with control and spAD groups (Fig. 17A). For both TIA-1 and VCP mRNA levels, a significant increase was observed in rpAD subjects, in comparison with control and $\operatorname{spAD}$ subjects, as was observed for protein expression (Fig. 17B and C).

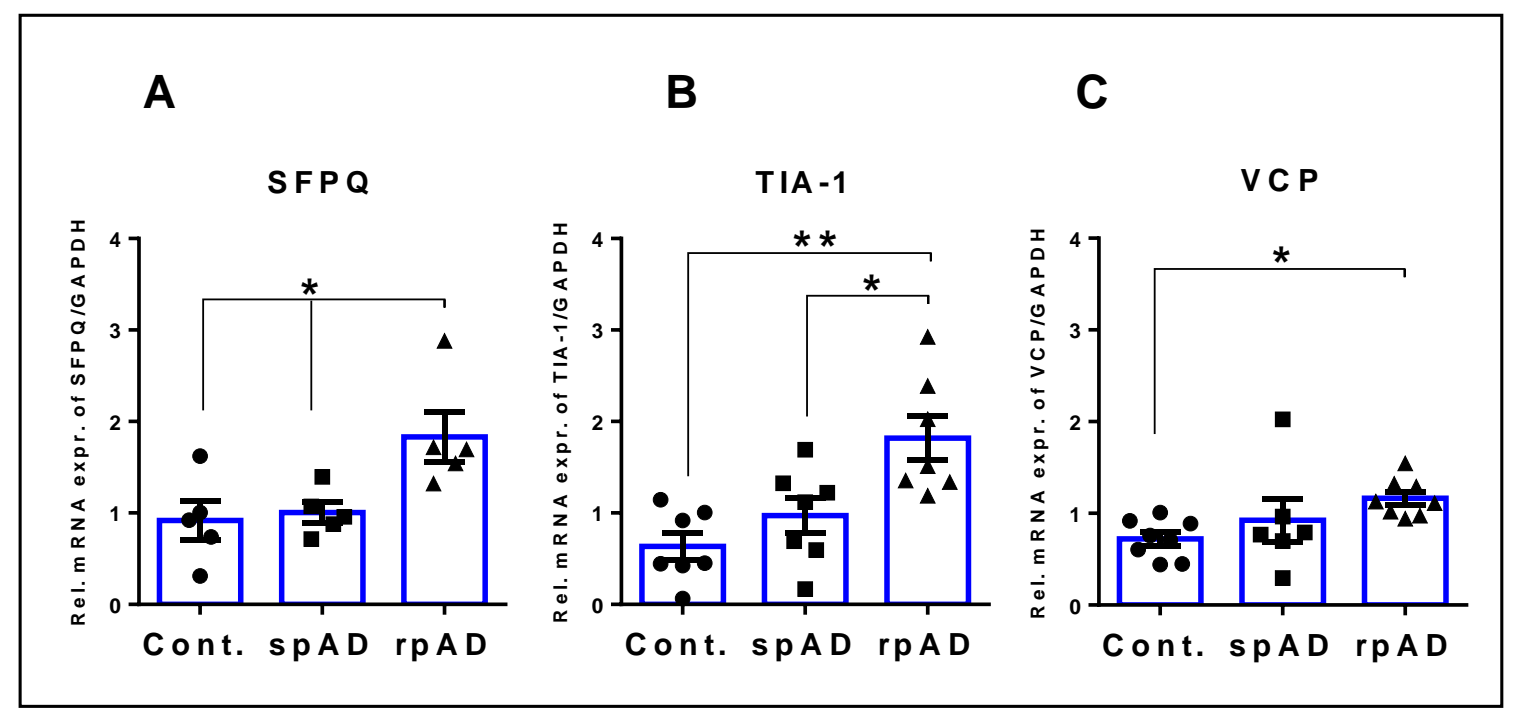

Figure 17: Expression of SFPQ, TIA-1, and VCP at mRNA level. A-C) Expression of SFPQ, TIA-1 and VCP at mRNA level was analysed in spAD, rpAD, and controls using qRT-PCR. The expression levels of mRNA were normalized to GAPDH. The comparative $C_{t}$ method $\left(2^{-\Delta \Delta C t}\right)$ was used for calculation of relative mRNA levels (Livak and Schmittgen, 2001). One-way ANOVA followed by Tukey post-hoc analysis for multiple comparisons, ${ }^{*} p<0.05,{ }^{* *} p<0.01,(n=5-8)$. 


\subsubsection{SFPQ mislocalization and co-localization with SG marker TIA-1 in the rPAD brain}

Mislocalization of several RBPs has been described as a pathological feature in many neurodegenerative diseases (Barmada et al., 2010; Bishof et al., 2018; Neumann et al., 2006; Vance et al., 2013). Immunoblotting analysis revealed dysregulation of SFPQ in the human brain from patients diagnosed as rpAD and the two SCJD subtypes. Next, we sought to explore the localization of SFPQ in the human brain. In order to examine the localization of SFPQ, we immuno-stained the brain tissues from spAD, rpAD, and control subjects with antibodies specific for SFPQ. For this analysis, a separate cohort was used and the clinical data of the fifteen cases are listed in the annexure data table (Table 13). In most cells of the control and spAD brains, SFPQ was localized in the nucleus (Fig. 18A) which is the typical localization of SFPQ, as reported previously (Lu et al., 2018; Meissner et al., 2000). Interstingly, in rpAD cases, SFPQ was massively depleted from the nucleus. A ring-shaped SFPQ was observed around the nucleus (Fig. 18A). Dislocation of SFPQ was observed in $91 \%$ cells in rpAD, compared with $51 \%$ in $\mathrm{spAD}$ and $43 \%$ in controls (Fig. 18B). These results demonstrate that nuclear depletion and dislocation of SFPQ occurred in both patient and control tissues; however, the dislocation rate was specifically higher in rpAD brains.

The mislocalization of SFPQ, particularly in the $\mathrm{rPAD}$ cases, raised an interesting question of whether or not nuclear depletion of SFPQ was associated with its cytoplasmic accumulation. Recently, the mislocalization of some nuclear factors has been linked with their cytoplasmic accumulation in the stress granules (Barmada et al., 2010; Vance et al., 2013). To test this possibility, we double-labelled the tissues from diseased and control subjects with primary antibodies specific for SFPQ (red) and TIA-1 (green): a classical marker of SGs (Fig. 18A). Sudan black was used to quench lipofuscin fluorescence, as differentiation of $S G$ reactivity from lipofuscin can be quite challenging (Liu-Yesucevitz et al., 2010; Vanderweyde et al., 2012). Treatment with Sudan black highlights consolidated cytoplasmic TIA-1 reactivity, which tends to show strong fluorescence in the SGs, compared with nuclear signal which is quenched by Sudan black. Hence, it's difficult to observe nuclear TIA-1 in these Sudan black-treated tissues (Fig. 18A). 
TIA-1 reactivity was also observed in control cases with normal cognition, as has been noted previously (Vanderweyde et al., 2012), and a partial co-localization was also observed with SFPQ (Fig. 18A). In case of SpAD subjects, a moderate colocalization was observed. For rpAD cases, a complete co-localization was demonstrated for SFPQ and TIA-1 in the perinuclear/cytoplasmic area (Fig. 18A).

To measure quantitative association between SFPQ and TIA-1, co-localization analysis was performed with ImageJ and FIJI (Coloc 2 plugin) software. Multiple methods were used to provide a quantitative measure of the extent of co-localization. We analysed the co-localization of SFPQ and TIA-1 using Pearson's correlation coefficient (rP) and Threshold Mander's coefficient (tM) (Bolte and Cordelieres, 2006), together with intensity correlation analysis (ICA). Intensity correlation analysis (PDM plots) showed highest degree of co-localization between SFPQ and TIA-1 in the rPAD brain, followed by SpAD and controls (Fig. 18A). A significant degree of colocalization was observed in rpAD cases, as judged from the two co-localization coefficients $\mathrm{rP}$ and $\mathrm{tM}$ (Fig. $18 \mathrm{C}$ and $\mathrm{D}$ ). The value of tM1 shows the overlap of TIA-1 channel pixels with SFPQ channel pixels, which is significantly higher in rpAD when compared with either spAD or control subjects. For tM2 (representing the overlap of SFPQ channel pixels with TIA-1 channel pixels), a trend was observed for rPAD cases, as well.

In summary, quantitative analysis of the brain-tissue staining demonstrated that colocalization between SFPQ and TIA-1 was observed in both SPAD and rPAD cases, with a stronger degree of co-localization in rpAD subjects. 


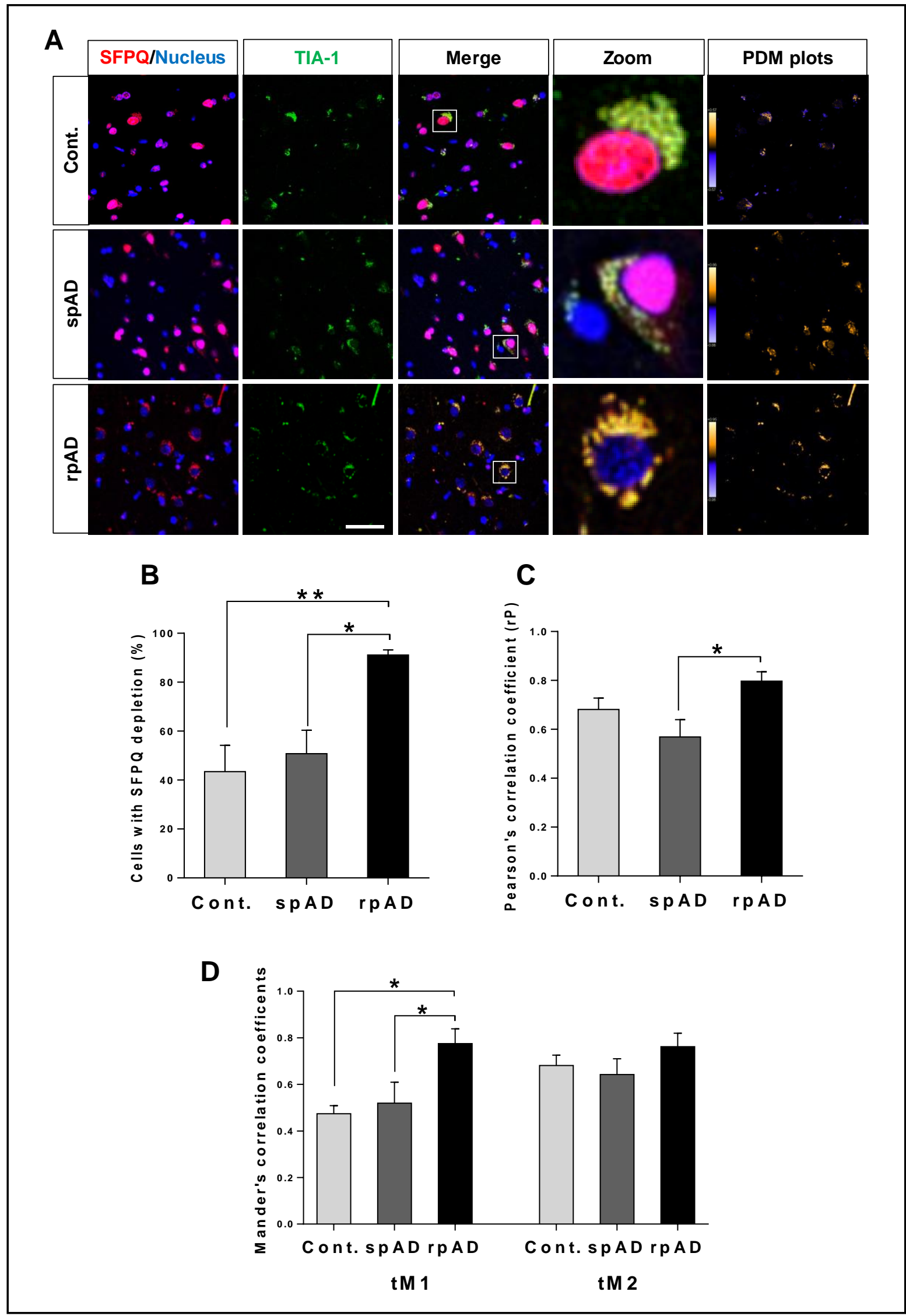

Figure 18: Dislocation/depletion of SFPQ from the nucleus and co-localization with the SG marker TIA-1 in the cytoplasm. A) Co-immunofluorescence of SFPQ (red) and TIA-1 (green) in the human brain of $\operatorname{spAD}(\mathrm{n}=$ $4)$, $\operatorname{rpAD}(n=4)$, and controls $(n=5)$. Cell nuclei were visualized with To-Pro-3 iodide staining (blue). Repre- 
sentative images are shown (scale bar $=50 \mu \mathrm{m}$ ). Cells are also shown at higher magnification for a closer look in each group. Intensity correlation analysis (ICA) was performed with ImageJ, showing PDM plots, which are representing highest overlap in rpAD cases, followed by SpAD and controls. B) Quantification of the cells with SFPQ dislocation $(n=150)$. C) Pearson's correlation coefficient $(r P)$ graph, representing co-localization coefficient between SFPQ and TIA-1. The co-localization between SFPQ (red) and TIA-1 (green) was analysed with FIJI (Coloc 2 plugin) software. D) Co-localization analysis with Threshold Mander's correlation coefficients (tM). The value of tM1 shows the overlap of TIA-1 channel pixels with SFPQ channel pixels, and tM2 represents the overlap of SFPQ channel pixels with TIA-1 channel pixels. One-way ANOVA followed by Tukey post-hoc test for multiple comparisons, ${ }^{*} p<0.05,{ }^{* *} p<0.01$.

\subsubsection{SFPQ is co-localized with phospho-tau in neurofibrillary tangles in the rpAD brain}

Cytoplasmic co-aggregation of some splicing factors with tau protein has been reported in both sporadic and familial cases of AD (Bai et al., 2013; Bishof et al., 2018; Diner et al., 2014). To test this possibility, the relationship of SFPQ with tau tangles was explored by immunohistochemical analysis. In frontal cortex tissues from the control subjects, immunoreactivity for SFPQ and phospho-tau (S199) was observed predominantly in the nucleus, with a strong degree of co-localization between both proteins. To further investigate this relationship, typical cells were investigated at high magnification in the indirect immunofluorescence micrographs (Fig. 19A). In the human brain of SpAD patients, a strong signal was observed for SFPQ in the nucleus and for phospho-tau in the cytoplasm, with a partial overlap between both in the nuclear and cytoplasmic regions (Fig. 19A). Interestingly, in rpAD subjects, there was a dislocation of SFPQ from the nucleus and immunoreactivity was observed in the cytoplasm. A significant co-localization was evident with phospho-tau tangles in the cytoplasm (Fig. 19A).

In order to measure the association between SFPQ and phospho-tau quantitatively, co-localization analysis was performed with ImageJ and FIJI (Coloc 2 plugin) software. Again, multiple methods were used to investigate the extent of co-localization between SFPQ and phospho-tau. Partial co-localization was observed between SFPQ and phospho-tau in the nuclear region in SPAD cases, as was evident from both co-localization methods ( $\mathrm{PP}$ and tM coefficients). In rpAD cases, two interesting findings were observed. Firstly, co-localization between SFPQ and phospho-tau was significantly increased compared with spAD group (Fig. 19B and C). Secondly, this 
co-localization was primarily in the cytoplasmic region rather than predominant colocalization in the nucleus, which is typically seen in controls.

In summary, co-immunofluorescence analysis of the affected brain tissues revealed extranuclear distribution of SFPQ and phospho-tau, and co-localization in the cytoplasm, contrary to the predominantly nuclear-based co-localization observed in control cases. 

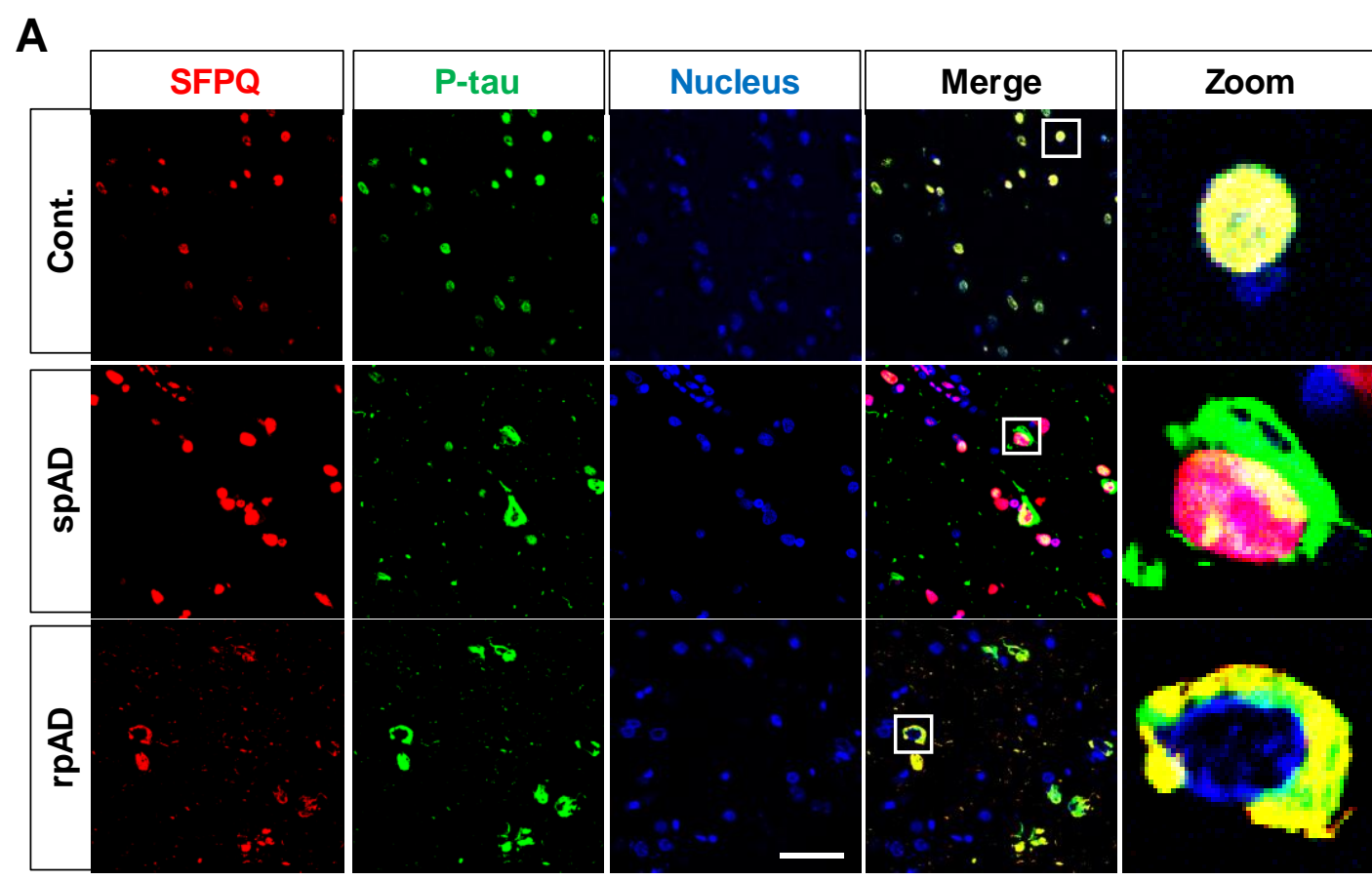

B

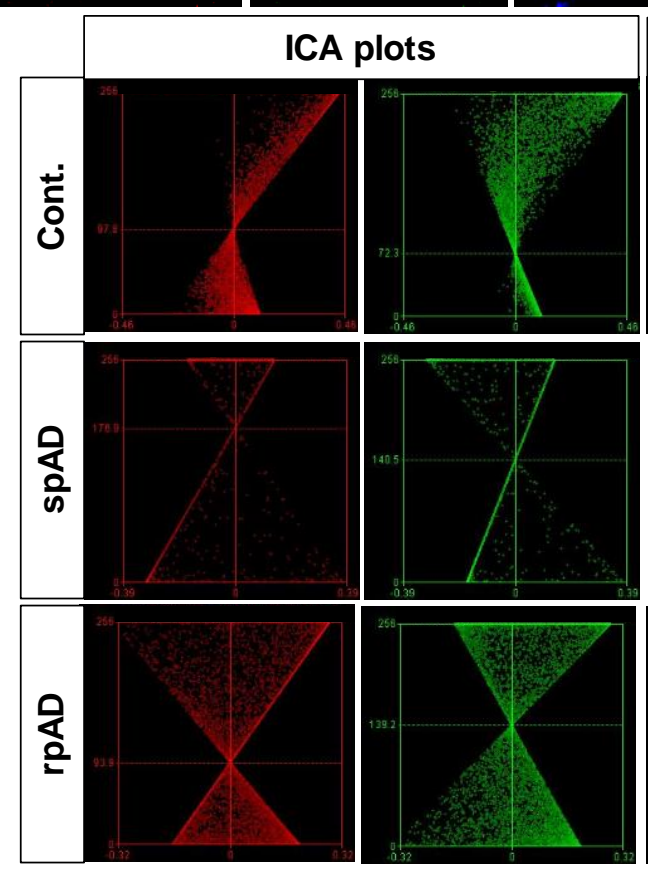

Freq. CP

PDM plots

B

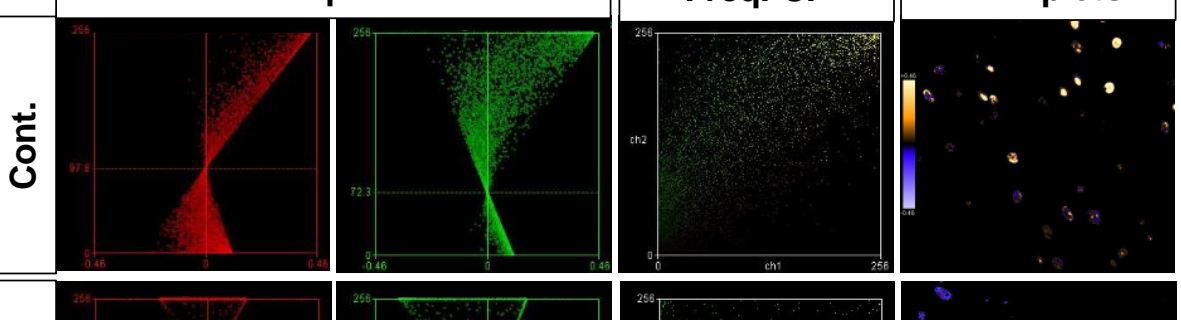

C
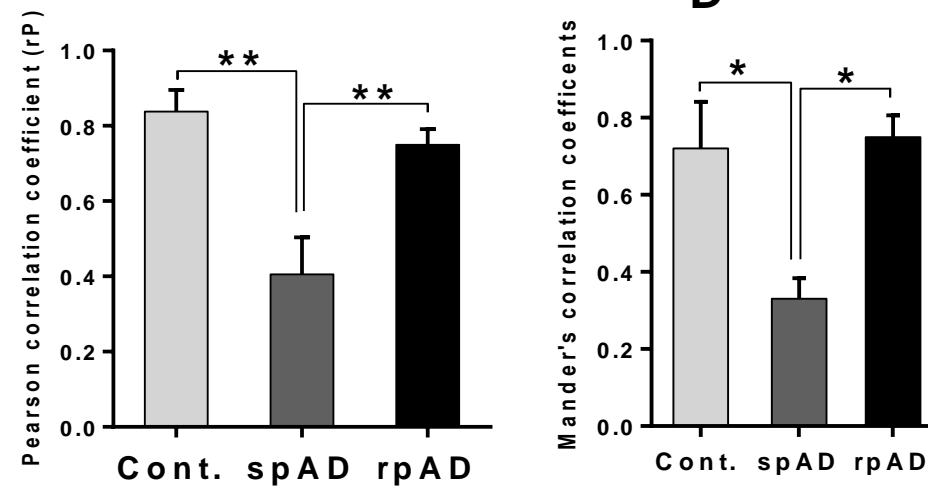

tM 1

tM 2 


\section{Results}

Figure 19: Co-localization of SFPQ with phospho-tau tangles. A) Representative images stained with $\alpha$ phospho-tau (S199) (green) and SFPQ (red) antibodies (scale bar $=50 \mu \mathrm{m}$ ), counter-stained with To-Pro-3 iodide to visualize the nuclei (blue). In control subjects $(n=3)$, a strong degree of co-localization between SFPQ and phospho-tau was observed in the nucleus at high magnification. In $\operatorname{spAD}$ tissues $(n=3)$, partial co-localization between SFPQ and phospho-tau was observed in the nuclear region. In rpAD cases $(n=3)$, a strong degree of co-localization was observed between SFPQ and phospho-tau in the cytoplasm, contrary to the nuclear colocalization which was seen in the control subjects. B) Intensity correlation analysis (ICA) was performed with Image-J (WCIF plug-in), ICA plots, frequency co-localization plots and PDM plots are displayed for control, spAD and rpAD. C) Pearson's correlation coefficient (rP) showing significant co-localization between SFPQ and phospho-tau in rpAD and control cases, as compared with spAD cases. D) Threshold Mander's correlation coefficients (tM) representing similar significant co-localization between SFPQ and phospho-tau in rpAD, in comparison to spAD cases. Graphs were prepared with GraphPad Prism (6.01) using One-way ANOVA followed by the Tukey post-hoc test for multiple comparisons, ${ }^{*} p<0.05,{ }^{* *} p<0.01$.

The formation of pathological tau tangles is associated with hyperphosphorylation of the tau protein in the $A D$ brain. Expression of total tau and phosphorylated tau (S199) was assessed by immunoblotting analysis (Fig. 20A). Biochemically, no significant changes were observed in the levels of total tau (tau-5), and phospho-tau to tau ratio (Fig. 20B and D). The levels of phosphorylated tau (S199) were significantly increased in the high molecular weight (HMW $=65-250 \mathrm{kDa}$ ) range in spAD cases, compared with control subjects (Fig. 20C). A trend was also observed for rpAD, but this relationship was not significant (Fig. 20C). 


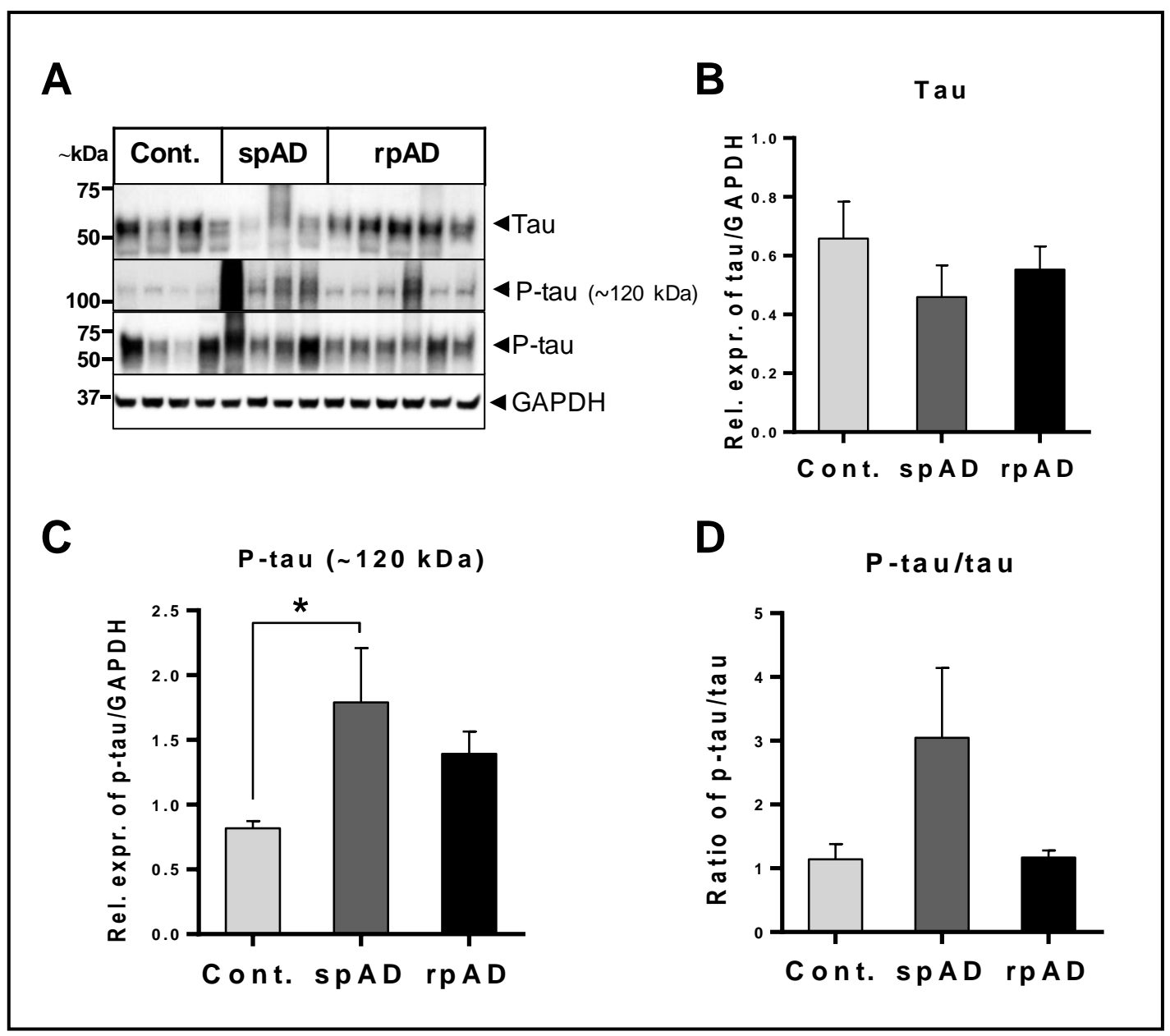

Figure 20: Differential expression analysis of tau and phospho-tau by immunoblotting. A) Representative immuno-blot images for total tau and phospho-tau expression from control, spAD, and rpAD frontal cortical tissue extracts. (B-D) Quantification of immunoblotting images for total tau and phospho-tau [low molecular weight-tau $(\mathrm{LMW}<65 \mathrm{kDa}$, and high molecular weight (HMW =65-250 kDa)]. GAPDH was used as a loading control. There were no significant changes observed for total tau and the ratio of phospho-tau to tau. High molecular weightrange band $(\sim 120 \mathrm{kDa})$ of phospho-tau was significantly increased in SpAD in comparison to control, while a trend was observed for other bands. Graphs were plotted by GraphPad prism (version 6.01). One-way ANOVA followed by Tukey post-hoc test for multiple comparisons were conducted, ${ }^{*} p<0.05$.

\subsubsection{Tau oligomers are co-localized with SFPQ in the rPAD brain}

Although cytoplasmic tau tangles are a burden for the cell, it has recently been shown that toxic, soluble oligomeric species of tau are the real culprits associated with cognitive decline, neuronal dysfunction, and death (Guerrero-Muñoz et al., 2015; Shafiei et al., 2017). In order to examine the association of SFPQ with oligomeric tau, we co-immuno-stained the cortical sections from control and rpAD subjects, given that there is significant association of SFPQ with tau tangles in the rpAD 
brain, using SFPQ and anti-oligomeric antibody for tau (T22). Almost no reactivity was observed for oligomeric tau in control cases (Fig. 21A). Interestingly, the coimmunofluorescence analysis revealed a change in the fluorescence pattern of SFPQ, with a high degree of association with oligomeric tau in the cytoplasmic region, in rpAD subjects (Fig. 21A).

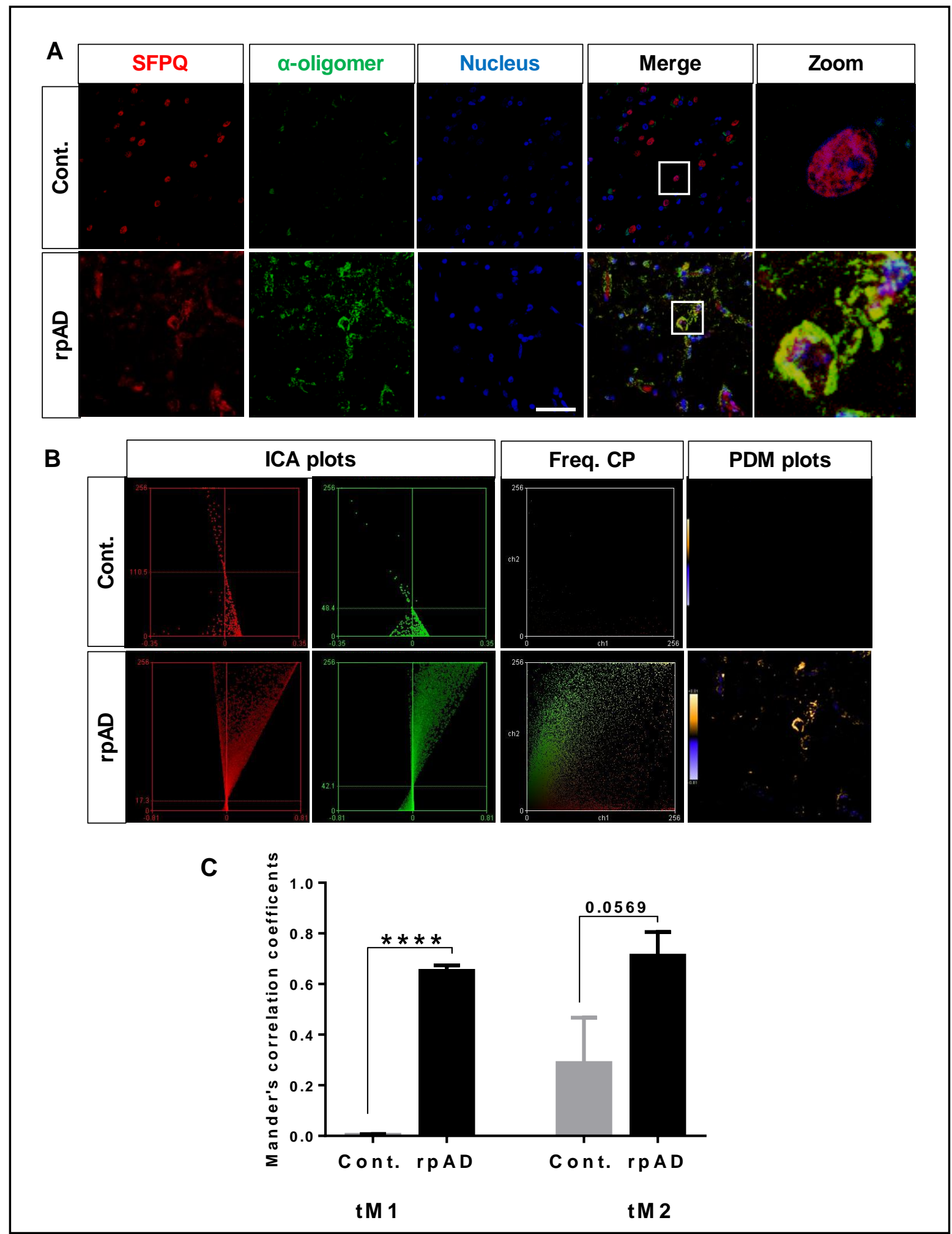


Figure 21: Co-localization of SFPQ with tau oligomers in the rPAD brain. A) Co-immunofluorescence images of control $(n=3)$ and $\operatorname{rpAD}(n=3)$ cortical sections stained with $\alpha$-SFPQ (red) and $\alpha$-Tau oligomeric antibody T22 (green). To-Pro-3 iodide was used for staining nuclei (blue), scale bar $=50 \mu \mathrm{m}$. There was no co-localization observed in control cases, while a significant co-localization was observed in rpAD subjects (yellow colour at higher magnification). B) Intensity correlation analysis showing ICA plots, frequency co-localization plots and PDM plots for both control and rpAD. C) Threshold Mander's correlation coefficients (tM1, tM2) showing significant association between SFPQ and oligomeric tau. Statistical significance was calculated by $t$-test, ${ }^{* * *} p<$ 0.0001 .

The degree of co-localization was estimated with ICA and Threshold Mander's correlation coefficients (tM1, tM2) (Fig. 21B and C). Significant co-localization was identified by both methods. Splicing factor proline and glutamine rich has a high PLD score and showed co-localization with tau oligomers. This potential interaction between SFPQ and tau oligomers might act as nidus for tau oligomerization and subsequent aggregation.

\subsection{Translational study of SFPQ in cellular models}

\subsubsection{Role of SFPQ towards stress axis}

Redistribution of SFPQ from the nucleus into the cytoplasm was observed in the staining of the human brain tissues particularly in rpAD subjects and a co-localization with TIA-1 in the cytoplasm. In order to investigate both the significance of this redistribution and the association with the stress granule protein TIA-1, a cellular model of stress was established in HeLa cells. A well-known oxidative stress inducer, sodium arsenite, was used for stress induction. Several studies have shown a clear link between arsenite-induced oxidative stress and $A D$ pathology in both animal models and a variety of cellular models (McEwen et al., 2005; Resende et al., 2008). Stress granules were analysed, where the viability of both control (untreated) and arsenitetreated cells under stress was not compromised (Fig. 40 in annexure data).

\subsubsection{Characterization of TIA-1-positive SGs}

For visualization of $S G s$, cells were stained after stress induction with the classical marker and core-nucleating factor of SGs, TIA-1. TIA-1 immunoreactivity was identified in both the cytoplasm and the nucleus, with predominance in the nucleus in the 
control (untreated) cells (Fig. 22A). Sodium arsenite treatment resulted in the formation of clearly defined cytoplasmic foci which positively stained TIA-1 in more than $80 \%$ of cells (Fig. 22A and D). To assess the expression of TIA-1 after stress induction, immunoblotting analysis was performed in both untreated (cont.) and arsenitetreated (stress) cells. A significant increase was observed in the intensity levels of TIA-1 after arsenite-induced cellular stress (Fig. 22B and C).

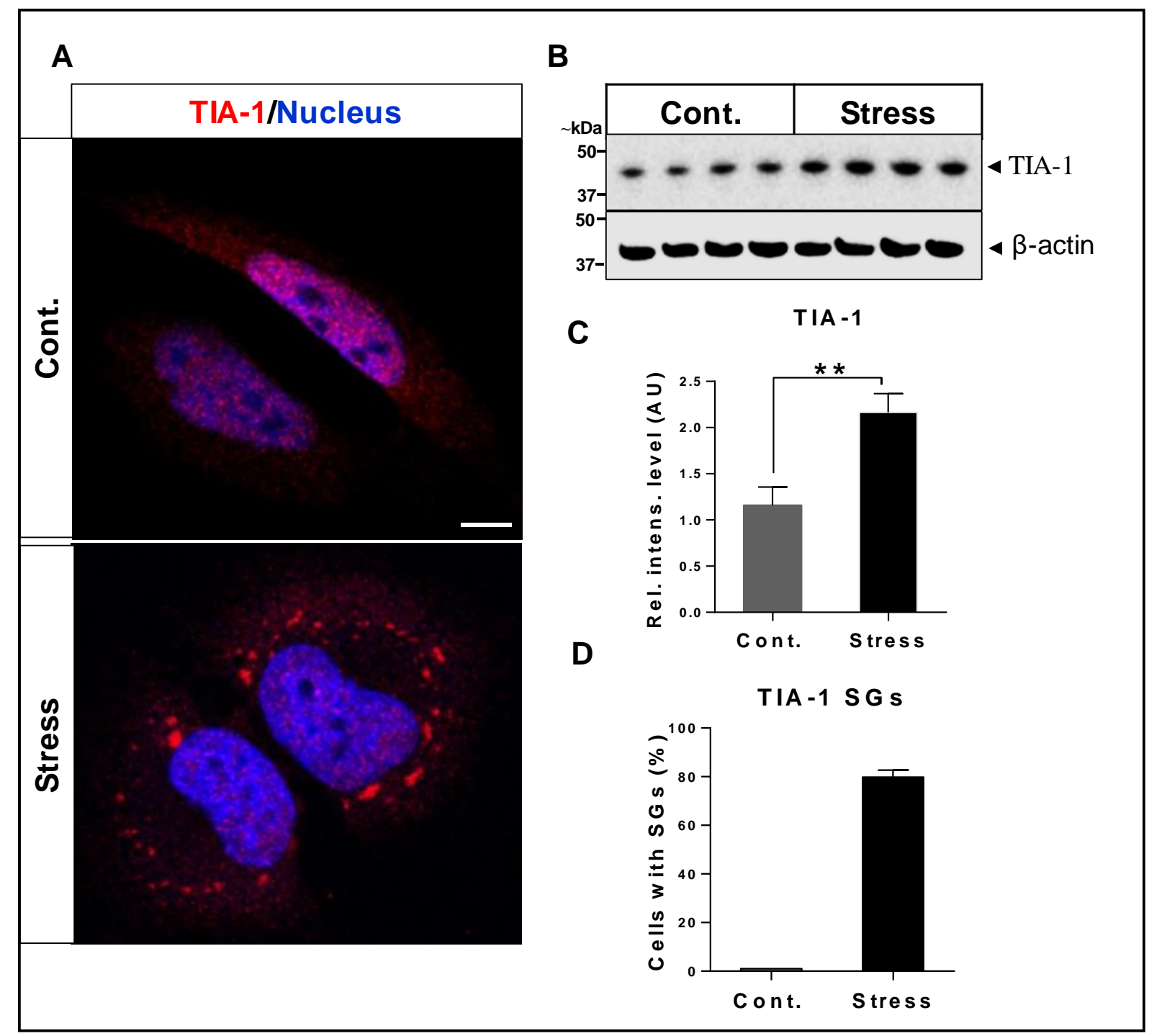

Figure 22: Sodium arsenite induces the formation of SGs in HeLa cells. A) HeLa cells were seeded on glass cover slips in 24-well plates $\left(5 \times 10^{4}\right.$ cells/well) for $24 \mathrm{hrs}$ in DMEM supplemented with $10 \%$ FBS and $1 \%$ PS. Cells were treated with sodium arsenite $(0.6 \mathrm{mM})$ at $37^{\circ} \mathrm{C}$ for $1 \mathrm{hr}$ followed by fixation with $4 \%$ PFA for $20 \mathrm{~min}$ at RT. Using primary antibody specific for TIA-1, stress granules were visualized by staining the classical marker of SGs TIA-1, followed by incubation with secondary antibody AlexaFluor 546 . The cells were counter-stained for visualization of nuclei and mounted with immuo-mount mounting medium, scale bar = $10 \mathrm{um}$. B and C) Levels of TIA-1 were determined by immunoblotting followed by densitometric analysis. Significance was estimated by $t$-test, ${ }^{* *} p$ $<0.01$. D) The cells positive for SGs were calculated with FIJI software. More than $80 \%$ cells were identified positive for SGs after arsenite treatment. 
A significant proportion of our knowledge on these cytoplasmic foci originates from studies in HeLa cells, including 154 reports published between 1999-2014, according to Aulas and Vande Velde (2015). Based on observations from the current study and a literature survey, HeLa cells were found to be the best representative of SGs. Hence, the HeLa cell line was preferred to further investigate the role of the target protein candidates in SG biology.

\subsubsection{Tau phosphorylation is increased after stress induction}

Numerous rodent studies focusing on the role of acute physiological or psychological stress have reported increased phosphorylation of tau after stress exposure (Feng et al., 2005; Korneyev, 1998; Lopes et al., 2016; Papasozomenos, 1996; Planel et al., 2001, Planel et al., 2004; Yanagisawa et al., 1999). Furthermore, several studies in cellular models have also demonstrated that oxidative stress leads to increased tau phosphorylation in neuronal cultures (Su et al., 2010; Zhu et al., 2005). Firstly, we investigated the levels of total tau after stress induction. There was no significant change observed for total tau levels between the control and stress-induced groups (Fig. 23A and C). For phospho-tau (S199), stress treatment induced significant increases in the levels of phosphorylation when compared with untreated control cells (Fig. 23A and D). This was found to be within the HMW range (65-250 kDa), suggesting tau aggregation under stressful conditions (Su, 2010).

To rule out the possibility that this observed increase in the tau phosphorylation in HeLa cells was cell-specific, the status of the tau phosphorylation was also investigated in the neuronal cell line SH-SY5Y after stress induction. Similarly, significant increases in the phosphorylated tau (S199) levels were identified in SH-SY5Y cells (Fig. 41 in annexure data). Overall, these results indicate that oxidative stress treatment increases tau phosphorylation in both cell lines tested. 


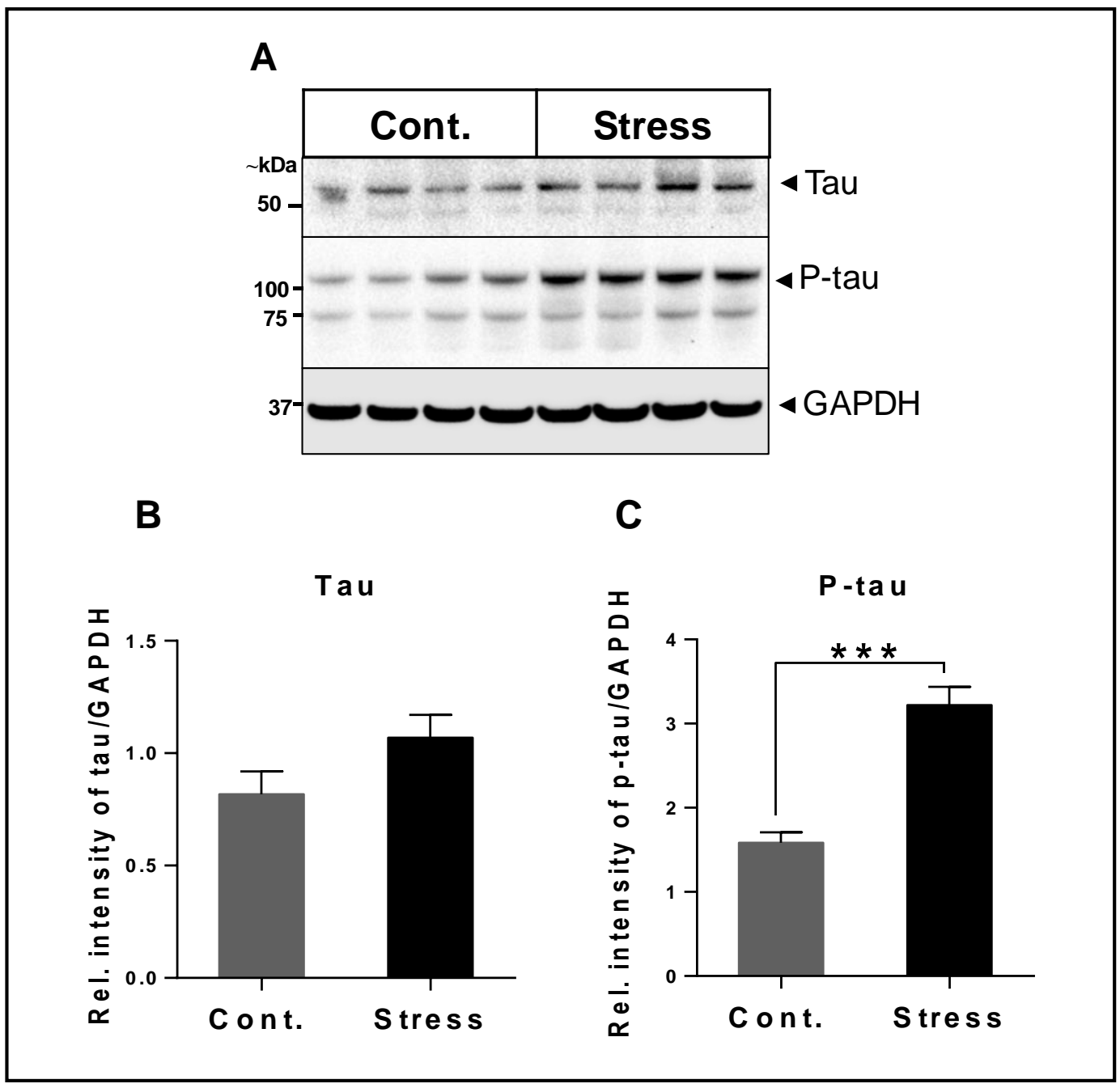

Figure 23: Stress-induced increase in tau phosphorylation. A) Representative immunoblots for total tau and phospho-tau in control (untreated) and stress (arsenite-treated) cells. Cells were plated in 6-well plates $\left(2 \times 10^{5}\right)$ for $24 \mathrm{hrs}$ and lysed in cell-lysis buffer supplemented with protease and phosphatase inhibitors. The expression of total tau and phospho-tau was analysed by immunoblotting. Intensity levels were normalized to GAPDH. B and C) The densitometric analysis was performed using Image Lab software. Statistical tests were applied in GraphPad prism (version 6.01) with significance ${ }^{* * *} p<0.001$.

\subsubsection{Tau and phospho-tau are recruited into SGs}

Previously, it has been reported that tau co-localizes with the SG markerTIA-1 in vivo (Vanderweyde et al., 2012). Next, we studied the relationship between tau and SGs in our cellular model of stress. We examined the association of phospho-tau and tau with TIA-1-positive SGs using immunocytochemistry. Labelling with total tau and phospho-tau antibodies revealed co-localization with TIA-1 cytoplasmic SGs (Fig. 24A and B, high magnification images). Untreated control cells showed a positive signal for tau-5 predominantly in the cytoplasm. For phospho-tau (S199), a 
positive signal was detected in the cytosol and nucleus, with predominance in the nucleus (Fig. 42 in annexure data), which was in-line with previous studies conducted in HeLa cells (Ibáñez-Salazar et al., 2017; Sjöberg et al., 2006). Such a signal was increased upon oxidative stress treatment. Overall, these findings determine that tau and phospho-tau are recruited into stress granules after stress exposure. The average number of SGs in each cell was estimated with FIJI software. We identified a higher number of phospho-tau-positive SGs compared with tau-positive granules (Fig. 24C). In addition to these observations, it should be noted that the size of the phospho-tau-positive granules was bigger in comparison to tau-positive SGs.

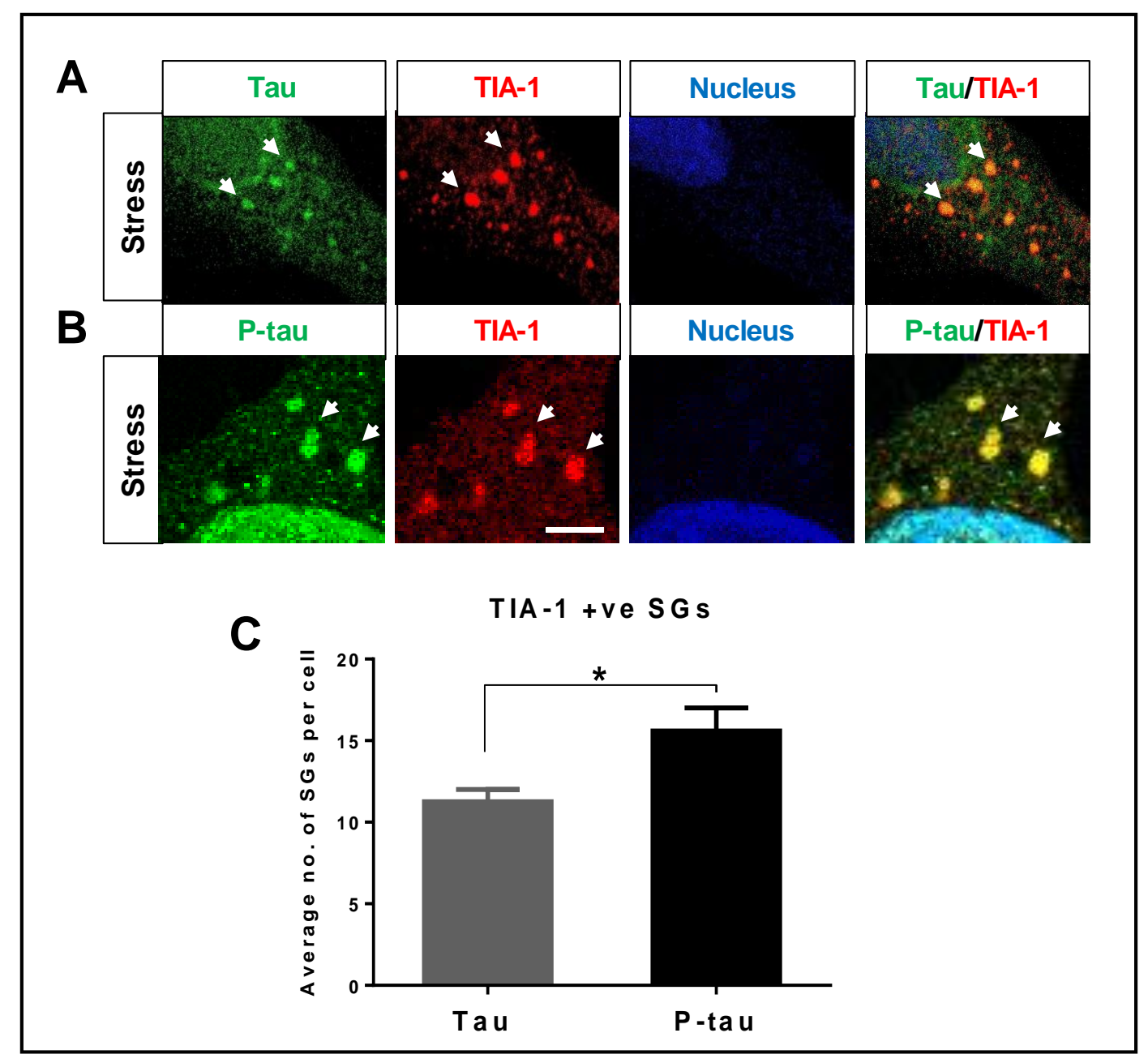

Figure 24: Tau and phospho-tau are recruited into SGs. A and B) Stress was induced with sodium arsenite as above and cells were co-immunoassayed with primary antibodies specific for total tau, phospho-tau and TIA-1, followed by incubation with AlexaFlour 488 and AlexaFlour 546 secondary antibodies. High magnification images showing the expression of tau/TIA-1 and phospho-tau/TIA-1 (Images with lower magnification are shown in annexure Fig. 42). Examples of SGs are indicated by the arrows. C) Average number of SGs per cell positive for TIA-1 was estimated both for tau and phospho-tau-positive stress granules using FIJI software. Significance was calculated by $t$-test, ${ }^{*} p<0.05$. 


\subsubsection{Endogenous SFPQ redistributes into the cytoplasm and assembles with SGs upon oxidative stress treatment}

To ascertain whether endogenous SFPQ forms SGs (as co-localization between SFPQ and TIA-1 was observed in the human brain), we studied the subcellular localization of SFPQ after stress induction. The presense of SFPQ in arsenite-induced cytoplasmic foci was observed by fluorescence microscopy. The protein SFPQ was mainly localized in the nucleus in the control (untreated) cells, which is the typical localization of SFPQ (Fig. 25A). The nuclear localization of SFPQ was not changed after arsenite treatment, but stress-induced redistribution/translocation of SFPQ following arsenite exposure into the cytoplasm and formation of granules in HeLa cells was observed (arrows, Fig. 25A).

To identify whether or not the SFPQ granules which could be observed in the cytoplasm were actually SGs, HeLa cells were co-stained with the SG marker TIA-1. Co-localization was detected between the SG marker and SFPQ (yellow foci in the cytoplasm) (Fig. 25B). The amount of cytoplasmic SFPQ signal was low because labelling only detected endogenous SFPQ, and SFPQ that was present in the cytoplasm was largely in the inclusions. Quantification of SFPQ-positive granules by FIJI software indicated $\sim 20-25$ SFPQ-positive stress granules per cell (Fig. 25C). Overall, these results revealed that stress induces cytoplasmic redistribution and formation of SFPQ inclusions, which co-localize with TIA-1 positive SGs.

Furthermore, we examined SFPQ levels before and after stress induction using immunoblotting analysis. A significant increase was identified in the SFPQ intensity levels after stress induction (Fig. 25D and E). Given the role of VCP in stress-granule dismantling and clearance, we next examined the levels of VCP after stress induction. A significant increase in VCP intensity levels was identified in stress-induced cells as compared with control cells (Fig. 25D and F). 


\section{Results}

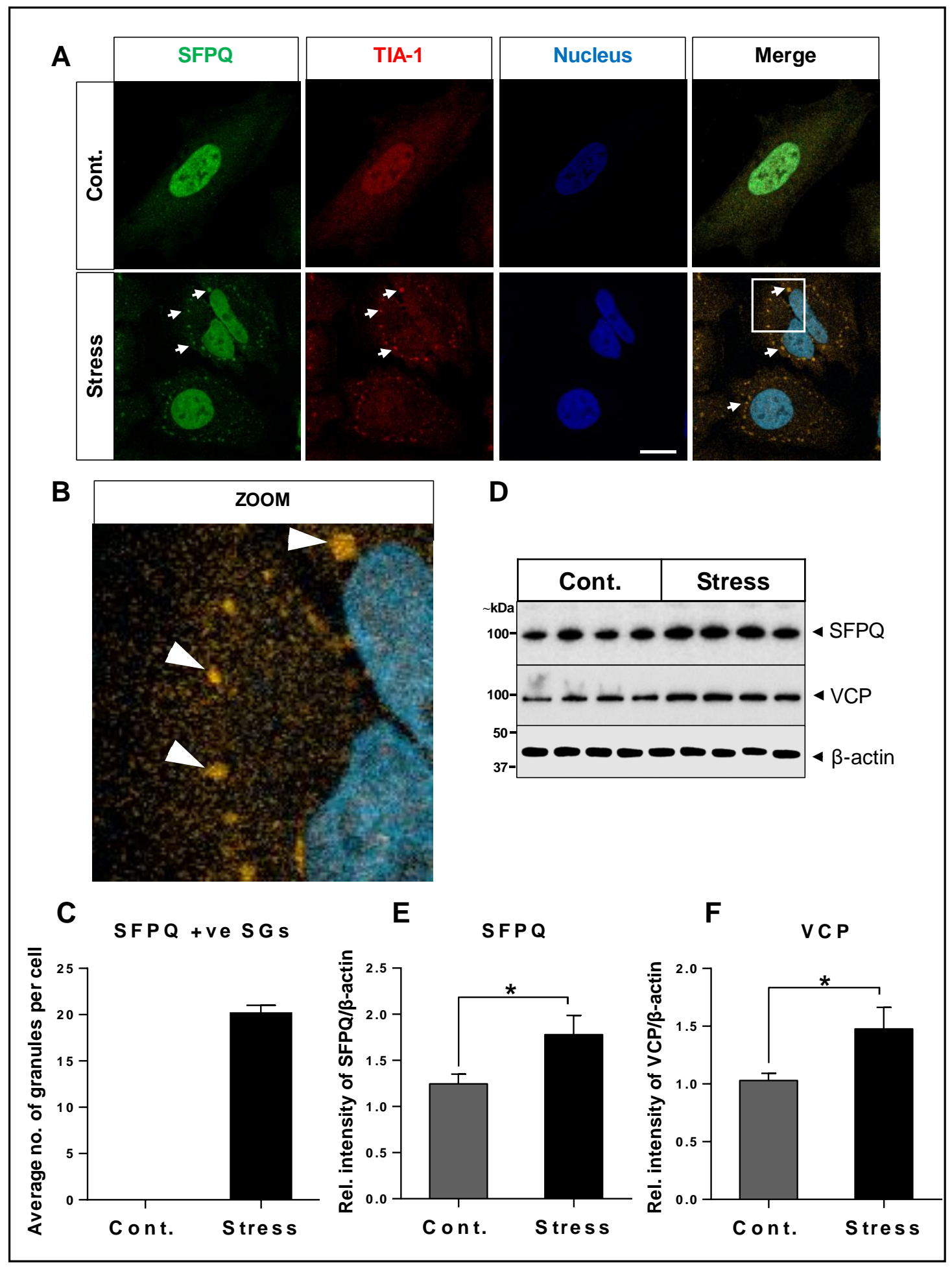

Figure 25: Recruitment of SFPQ into SGs after oxidative stress induction in HeLa cells. A) Localization of SFPQ (green) and TIA-1 (red) was visualized in sodium-arsenite-treated (0.6 mM; $60 \mathrm{~min}$ ) (stress) and untreated (control) HeLa cells using immunofluorescence microscopy. Cells were counter-stained to visualize nuclei, scale bar $=10 \mu \mathrm{m}$. B) Higher magnification image of "A" showing the overlap between SFPQ/TIA-1 in the cytoplasm. C) Average number of SFPQ-positive SGs per cell was calculated with FIJI software. D) Representative immunoblot images after stress treatment. Cells were seeded in 6 -well plates $\left(2 \times 10^{5}\right)$ for 24 hrs. Cells lysis was performed with cell-lysis buffer supplemented with protease and phosphatase inhibitors, and expression of SFPQ 
and VCP was analysed by immunoblotting. Intensity levels were normalized to $\beta$-actin. E and F) SFPQ and VCP densitometric analysis. Statistical tests (t-test) were applied in the GraphPad prism (6.01), ${ }^{*} p<0.05$.

According to a recently described model (Molliex et al., 2015), SG formation is dependent upon liquid-liquid phase separation (LLPS), a property of the RNA-binding proteins containing prion-like domains. We analysed SFPQ protein with catGRANULES algorithm, which predicts the propensity of a given protein to undergo phase separation, and the probability of forming granules. A score of 1.66 was identified for SFPQ showing high probability of SFPQ to form granules. We were also curious to know LLPS properties for TIA-1. Between the two proteins tested, SFPQ (1.66) exhibited a higher score for LLPS than TIA-1 (0.973) (Fig. 26A and B).

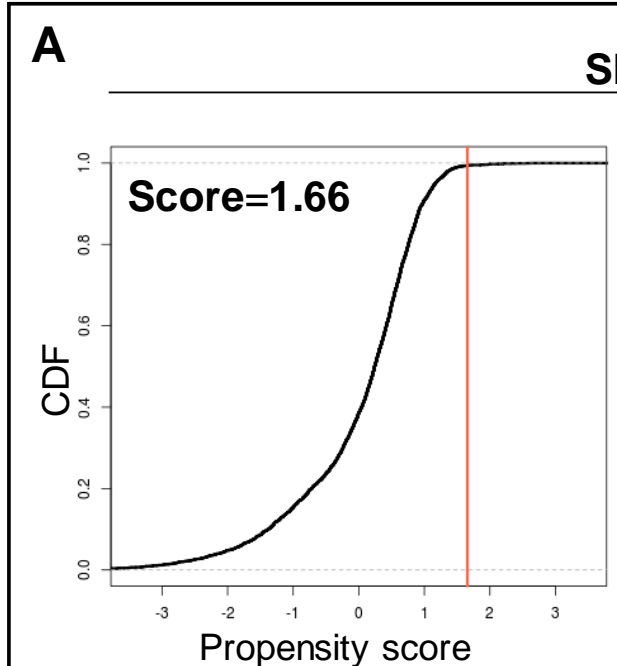

B

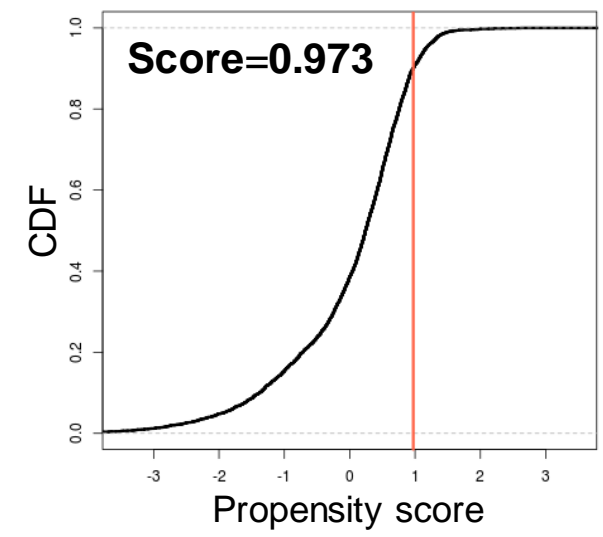

TIA-1
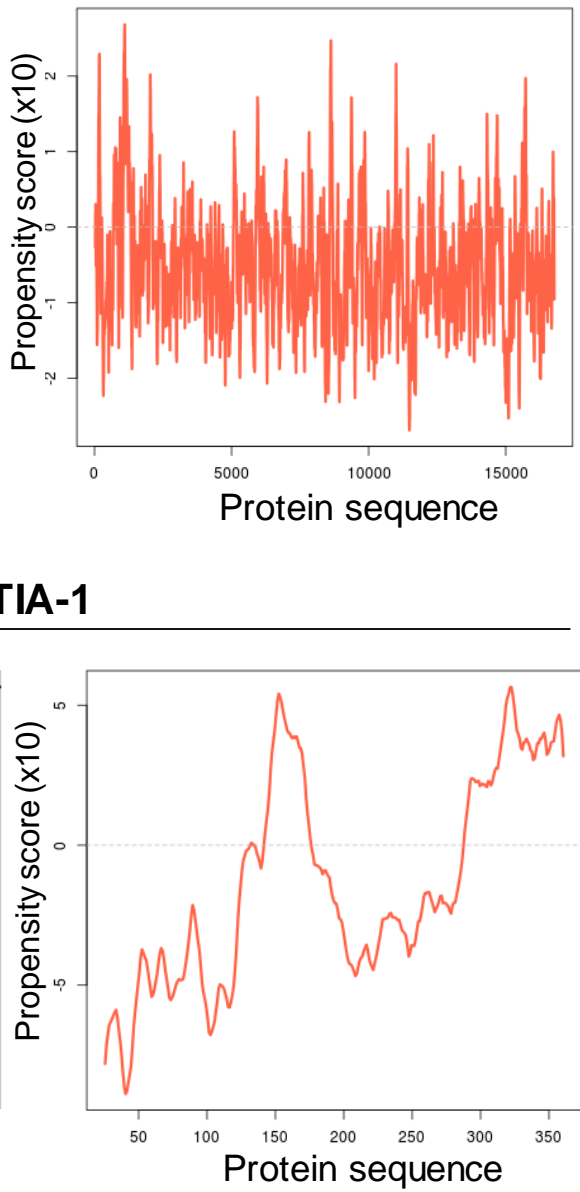

Figure 26: Liquid-liquid phase separation properties of SFPQ and TIA-1 assessed by catGRANULES algorithm. SFPQ exhibited a score of 1.66, followed by TIA-1: 0.973 , showing higher probability for oligomerization and granule formation. 


\subsubsection{SFPQ co-localizes with tau and phospho-tau in cytoplasmic granules}

Recruitment of SFPQ into SGs and localization of tau and phospho-tau in these SGs, raised the possibility that SFPQ and tau might co-localize in SGs and this functional interaction between SFPQ and tau might have implications for neurodegeneration. In the control cells, SFPQ was observed predominantly in the nucleus, while a redistribution was identified in the cytoplasmic-granules in treated (stress) cells. The immunoreactivity of SFPQ was increased after arsenite treatment (stress).

Labelling with tau-5 revealed majorly cytoplasmic staining in the control untreated cells (Fig. 27A). A predominant nuclear signal was detected for phospho-tau. Colocalization of SFPQ with both tau- 5 and phospho-tau was observed under basal conditions and in granules upon stress treatment (Fig. 27A and B). 


\section{Results}

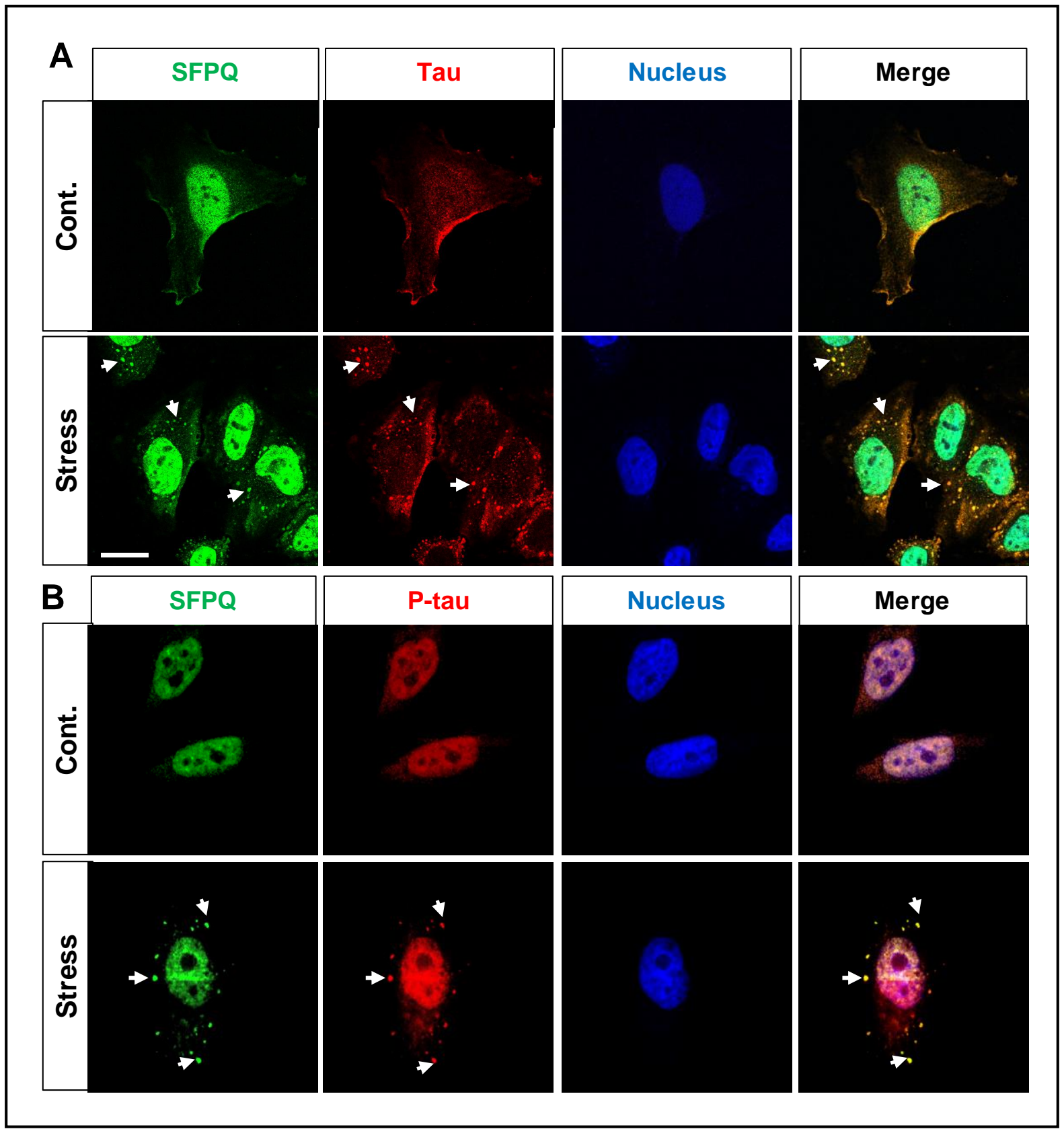

Figure 27: SFPQ co-localizes with tau and phospho-tau in SGs after sodium-arsenite-induced oxidative stress in HeLa cells. A and B) Localization of SFPQ (green), tau-5 and phospho-tau (red) was visualized in control (untreated) and stress (sodium-arsenite-treated) cells by immunofluorescence. Cells were counter-stained to visualize nuclei. Scale bar $=10 \mu \mathrm{m}$. Merged micrographs of cells (arsenite-treated) showing the overlap between SFPQ/tau and SFPQ/p-tau in the cytoplasmic granules.

\subsubsection{Biochemical characterization of stress granule components}

Increased intensity levels of SG-associated proteins after stress induction, raised the question of whether or not this was really an increase in their concentration or simply an increase in the intensity levels due to the consolidation of these proteins in SGs. To address this question, we performed subcellular fractionation of control and 
stress-induced HeLa cells using a Rapid, Efficient and Practical (REAP) method (Suzuki et al., 2010). This method is a very rapid (2 min), non-ionic detergent (NP40)-based purification method allowing very rapid fractionation of nuclear and cytoplasmic fraction, which is necessary given that nucleocytoplasmic transport of proteins is a rapid process in response to stress.

Immunoblotting analysis revealed an efficient subcellular fractionation with no cross contamination between nuclear and cytoplasmic fractions. The nuclear marker (BRD4) was not detected in the cytoplasmic fractions (Fig. 43 in annexure data). Conversely, the cytoplasmic marker (GAPDH) was not detected in the nuclear fraction (Fig. 43 in annexure data).

After an efficient fractionation, immunoblotting analysis was performed to investigate whether an increase in the intensity was observed after stress induction. For total tau levels, no significant changes were observed in line with the observations from total cell lysates analysis (Fig. 28A and B). Indeed, the REAP method showed a significant increase in the levels of phospho-tau in cytoplasmic and nuclear fractions, also in the HMW range (65-250 kDa: $\sim 120$ and $75 \mathrm{kDa})$, as was observed after stress treatment (Fig. 28A, C and D). 


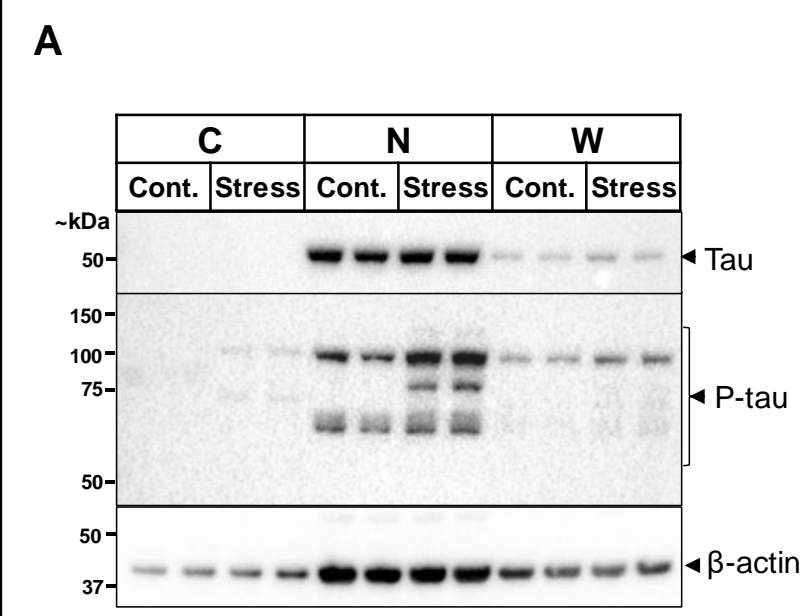

\section{B}

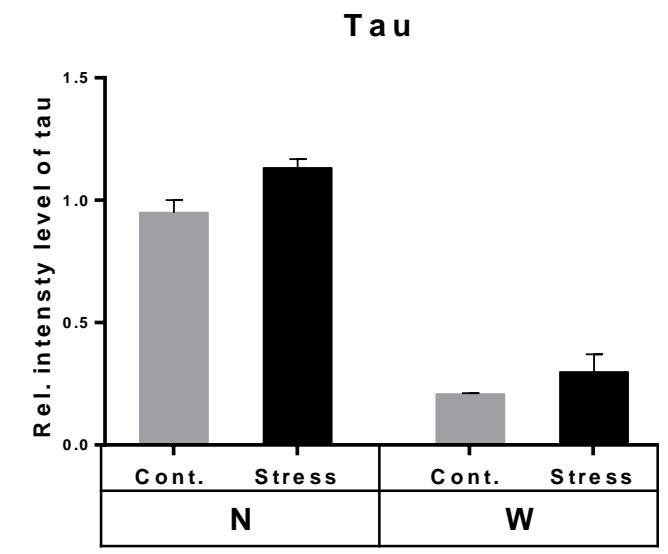

C

$$
\text { P-tau }
$$

D
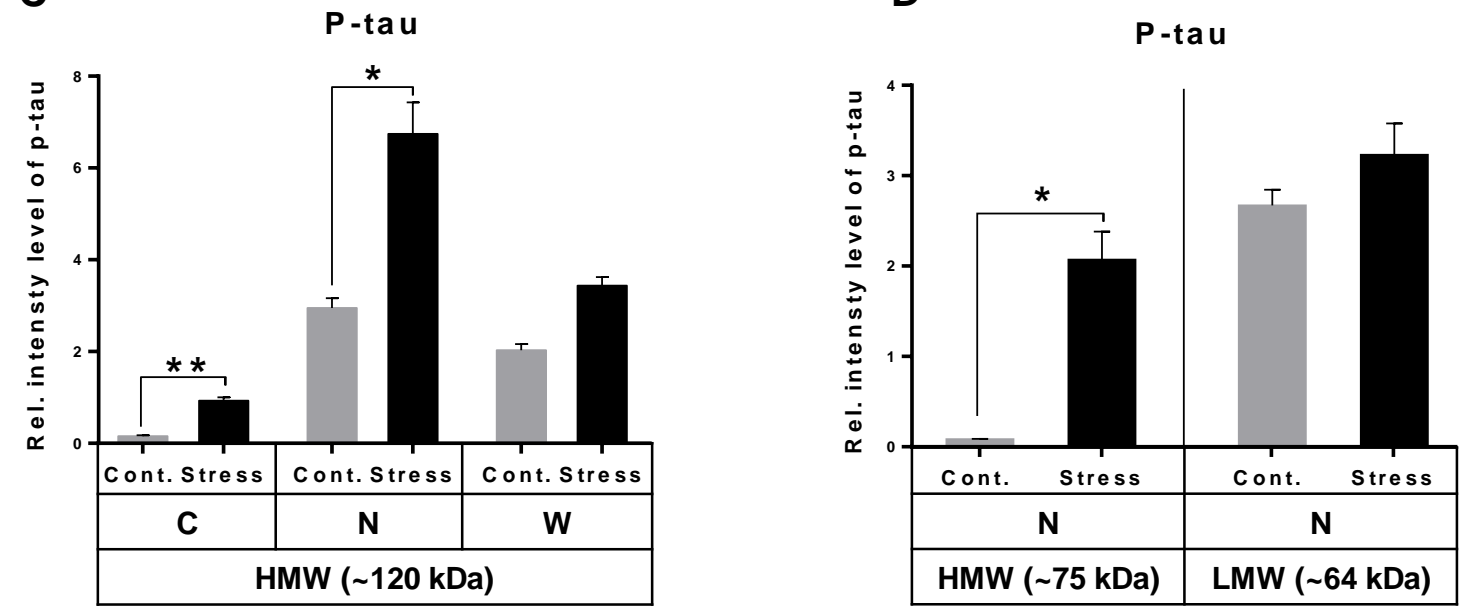

Figure 28: Increased tau phosphorylation and cytoplasmic accumulation after stress treatment. A) HeLa cells were treated with sodium arsenite $(0.6 \mathrm{mM}, 60 \mathrm{~min})$. Lysates from control (untreated) and stress (arsenitetreated) cells were separated into nuclear and cytoplasmic fractions by REAP method, which were analyzed by immunoblotting with total tau (tau-5) and phospho-tau (S199) antibodies. Intensity values were normalized to $\beta$ actin. Isolated fractions were abbreviated as $\mathbf{C}$ : cytoplasmic extract, $\mathbf{N}$ : nuclear extract, and $\mathbf{W}$ : whole cell extract. B-D) Quantification of all above proteins showing significantly increased intensity levels for phospho-tau as calculated by $t$-test, ${ }^{*} \mathrm{p}<0.05,{ }^{* *} \mathrm{p}<0.01$.

The REAP method also demonstrated significant increases in the intensity levels of TIA-1 in the nuclear fraction after stress induction as well as a slight increase in the whole cell fraction (Fig. 29A and B). For SFPQ, a moderate increase was also evident in both the nuclear and whole cell fractions (Fig. 29A and C). 


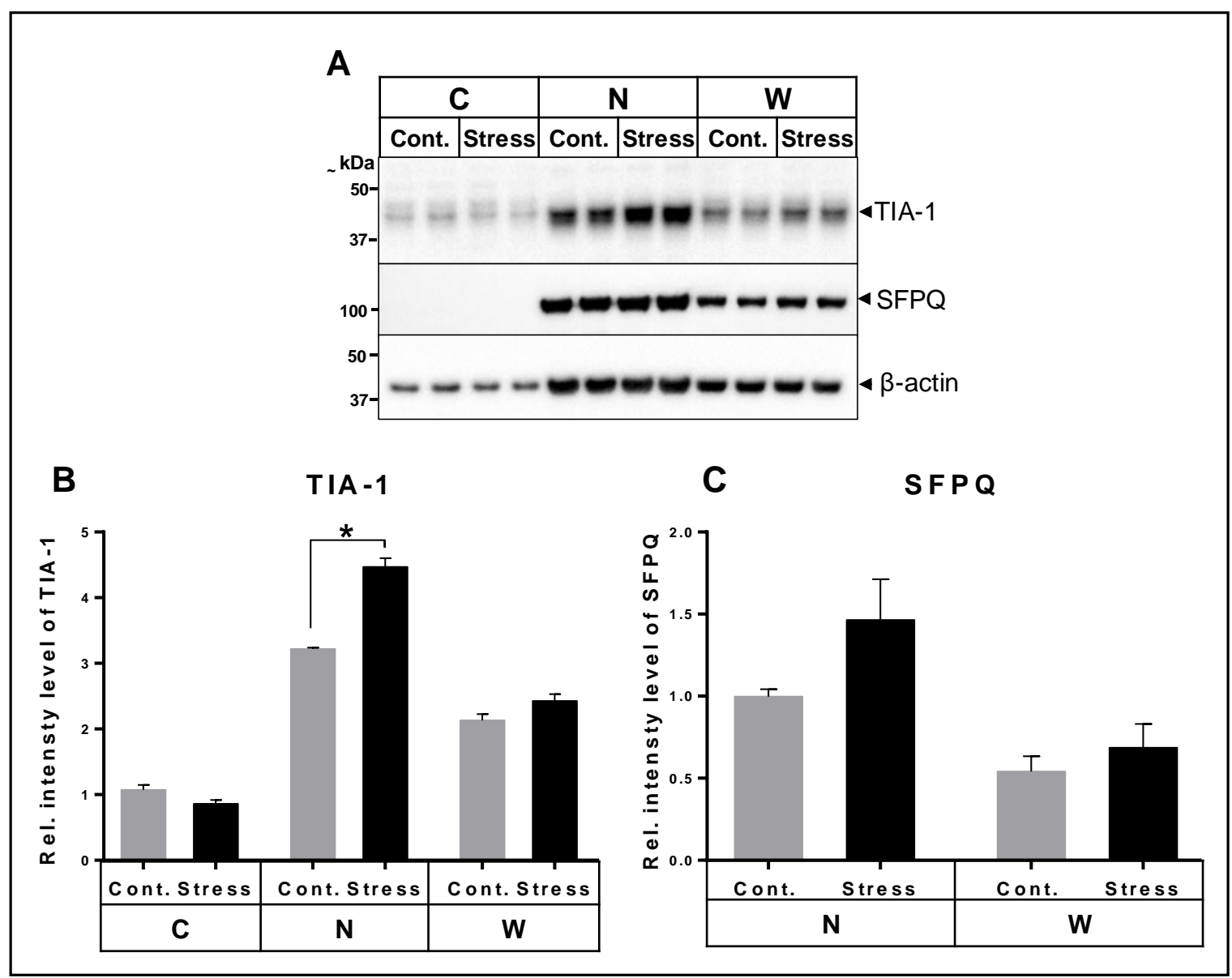

Figure 29: Expression of TIA-1 and SFPQ after subcellular fractionation. A) Stress was induced by treatment with sodium arsenite $(0.6 \mathrm{mM}, 60 \mathrm{~min})$. Lysates from control (untreated) and stress (arsenite-treated) cells were separated into nuclear and cytoplasmic fractions by REAP method, which were analyzed by immunoblotting with TIA-1 and SFPQ antibodies. Protein amounts were normalized to $\beta$-actin. Subcellular fractions were abbreviated as $\mathbf{C}$ : cytoplasmic extract, $\mathbf{N}$ : nuclear fraction, and $\mathbf{W}$ : whole cell lysate. $\mathbf{B}$ and $\mathbf{C})$ The densitometric analysis of TIA-1 and SFPQ showing significantly increased intensity levels for TIA-1 in the nuclear fraction particularly, as were calculated by $t$-test, $p$-value ${ }^{*} \mathrm{p}<0.05$.

\subsubsection{Role of SFPQ in the tau axis}

\subsubsection{SFPQ downregulation induced by human tau expression}

From cell staining (Fig. 27A), co-localization was observed between SFPQ and tau in the cytoplasm. Furthermore, Braak stage-dependent reduction in levels of SFPQ in the entorhinal cortex (EC) has been reported in the human brain (Ke et al., 2012). In order to find out a direct interaction of tau on SFPQ, we expressed human wild-type (WT-tau) and mutant tau (P301L-tau) transiently in HeLa cells. 
To assess the expression of tau after transfections, immunoblotting analysis was performed with antibodies specific for total tau (tau-5) and phospho-tau (S199) at 24and $48 \mathrm{hrs}$ post-transfection. Protein extracts from transfected cells showed an increased level of total tau and its phosphorylated form (Fig. 30A). The densitometric analysis revealed no significant differences in the ratio of phospho-tau to tau between WT-tau and P301L-tau transfected cells (Fig. 30B). A trend of increased phosphorylation could be noted for WT-tau-transfected extracts. Interestingly, expression of human tau led to a significant reduction in the levels of SFPQ, as assessed by immunoblotting at $48 \mathrm{hrs}$ post-transfection in WT-tau transfected cells, compared with control (Fig. 30A and C). For P301L-tau expressing cells, a decreasing trend was also observed at the $48 \mathrm{hrs}$ post-transfection. There were no significant changes observed at $24 \mathrm{hrs}$ post-transfection. For TIA-1 protein, no significant changes could be identified under any of these conditions (Fig. 30A and D).

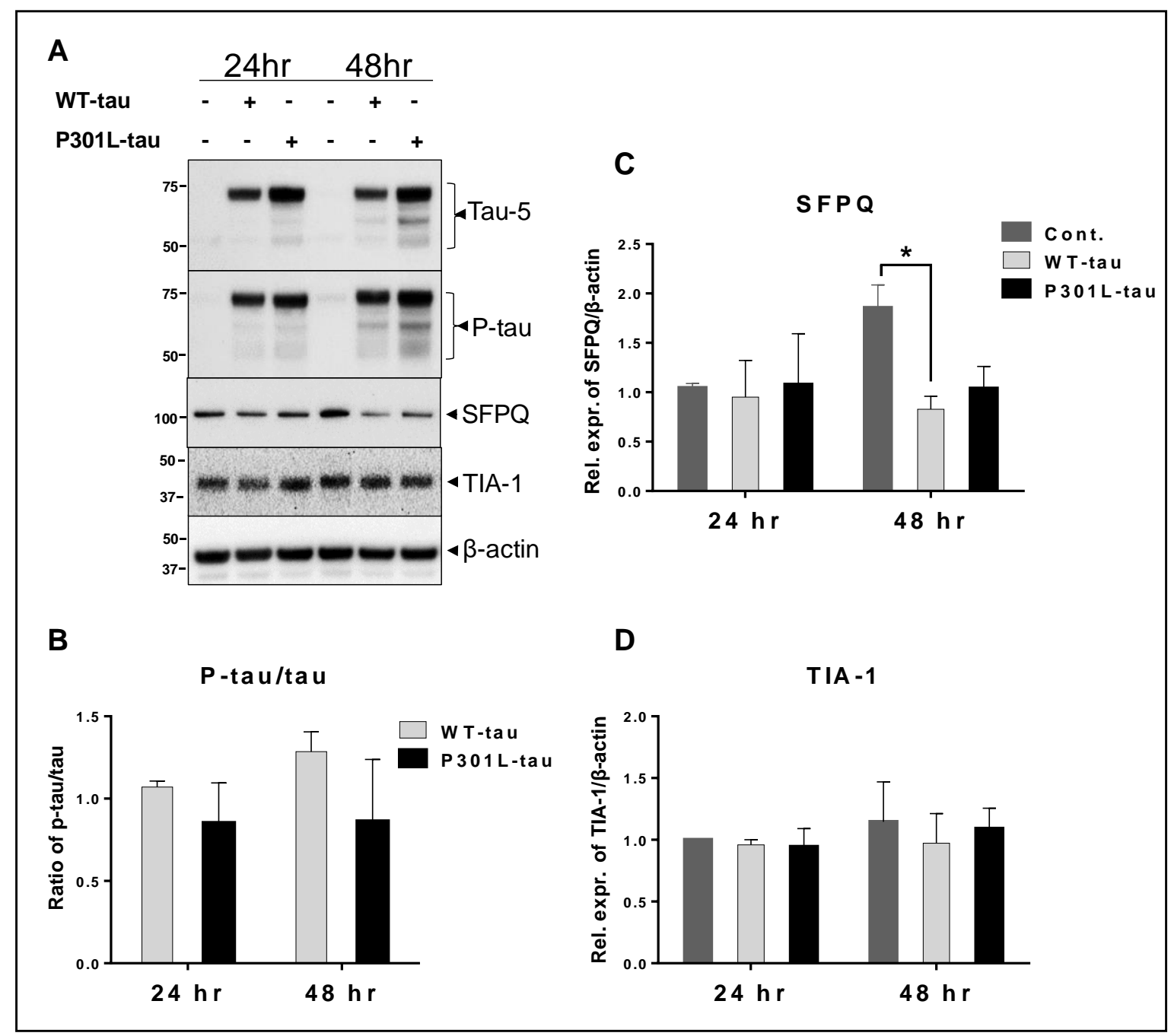


Figure 30: Tau regulates SFPQ levels in HeLa cells detected by immunoblotting. A) Representative immunoblots for tau, phospho-tau, SFPQ and TIA-1 after transient transfection of WT-tau or P301L-tau. B) Total protein lysates from transfected HeLa cells and control cells were subjected to immunodetection using tau-5 and phospho-tau (S199) antibodies. C) The levels of SFPQ were also determined after tau expression. Protein expression was normalized to $\beta$-actin. The densitometric analysis revealed significant decrease in the levels of SFPQ in WT-tau-expressing cells at 48 hrs post-transfection. D) For TIA-1, no significant differences were observed after transient expression of tau compared with transfection corresponding control. Significance was estimated with one-way ANOVA followed by Tukey post-hoc test for multiple comparisons, ${ }^{*} p<0.05$.

Cell viability was significantly reduced after tau expression in both WT- and P301Ltau-expressing cells, with more robust changes in WT-tau-expressing cells at $48 \mathrm{hrs}$ post-transfection (Fig. 44 in annexure data). In summary, immunoblotting analysis indicated that tau has a direct effect on SFPQ in the form of reduction, which coincides well with the massive reduction of SFPQ observed in the rpAD and SCJD patient's brains.

\subsubsection{Proteomic changes associated with SFPQ downregulation after human tau-expression}

Immunoblotting analysis after human tau expression in the cells indicated a direct effect of tau on SFPQ in the form of reduction. To determine the combinatorial effect of tau toxicity and SFPQ reduction in the cells, we used a quantitative SWATH labelfree proteomics technology. Quantitative MS-based proteomics has emerged as a powerful technology for investigating mammalian signalling pathways (Huang et al., 2015; Liu et al., 2017). Proteomic alterations after tau expression could provide novel insights into the mechanisms linking tau-pathology to SFPQ dysregulation.

In total, 3597 proteins were identified quantitatively at a critical FDR of $1 \%$. To identify differentially expressed proteins (DEPs), bioinformatic analysis was performed. Proteins with a corrected $\mathrm{BH}$ p-value $<0.05$ were considered differentially expressed, compared with controls (Fig. 31A and B). Significantly DEPs (314) were divided into two sets: the first consisted of 63 up-regulated proteins, and the second consisted of 251 down-regulated proteins (Tables 16 and 17 in annexure data).

To get deeper insights into the molecular mechanisms associated with significantly modulated proteins, a number of bioinformatic tools were used to examine enriched 
functional categories and pathways. Firstly, hierarchical clustering and enrichment analysis was performed using Fisher's exact test by Perseus software, in order to have an overview of the significantly enriched functional profile. As shown in Fig. $31 \mathrm{C}$, the significantly regulated proteins were clustered hierarchically across all the samples (columns) and the significantly regulated proteins (rows). Interestingly, the main branch point in the columns separated the tau-expressing cells (Fig. 31C, Lanes 1-12) from controls (mock-transfected or non-transfected controls) (Fig. 31C, Lanes 13-24). No significant differences were observed between WT-tau and P301L-tau expressing phenotypes.

The significantly regulated proteins (rows) were grouped into two main sub-clusters. Cluster 1 included proteins that were upregulated in tau-expressing cells (both WTtau and P301L-tau). The functional enrichment analysis of cluster 1 by Fisher's exact test indicated highly significant enrichment for substrate specific transporter activity and immune system processes (Fig. 31C: right panel). The downregulated proteins (cluster 2) showed enrichment for functional terms associated with "structural constituent of ribosome", and "SRP-dependent co-translational protein targeting to membrane" among others (Fig. 31C: right panel). Additionally, an overrepresentation analysis was performed using WEB-based Gene SeT AnaLysis Toolkit (WebGestalt). By classifying the 314-DEPs (Liao et al., 2019) based on their "Biological Process", 202 proteins were identified as being related to the metabolic processes, 180 proteins linked to biological regulation, and 139 attributed to be involved in response to the stimulus (Fig. 31D). For Molecular Function, 228 proteins were assigned to the protein-binding category, with 109 attributed to be related to nucleic-acid binding, and 107 to ion binding. 


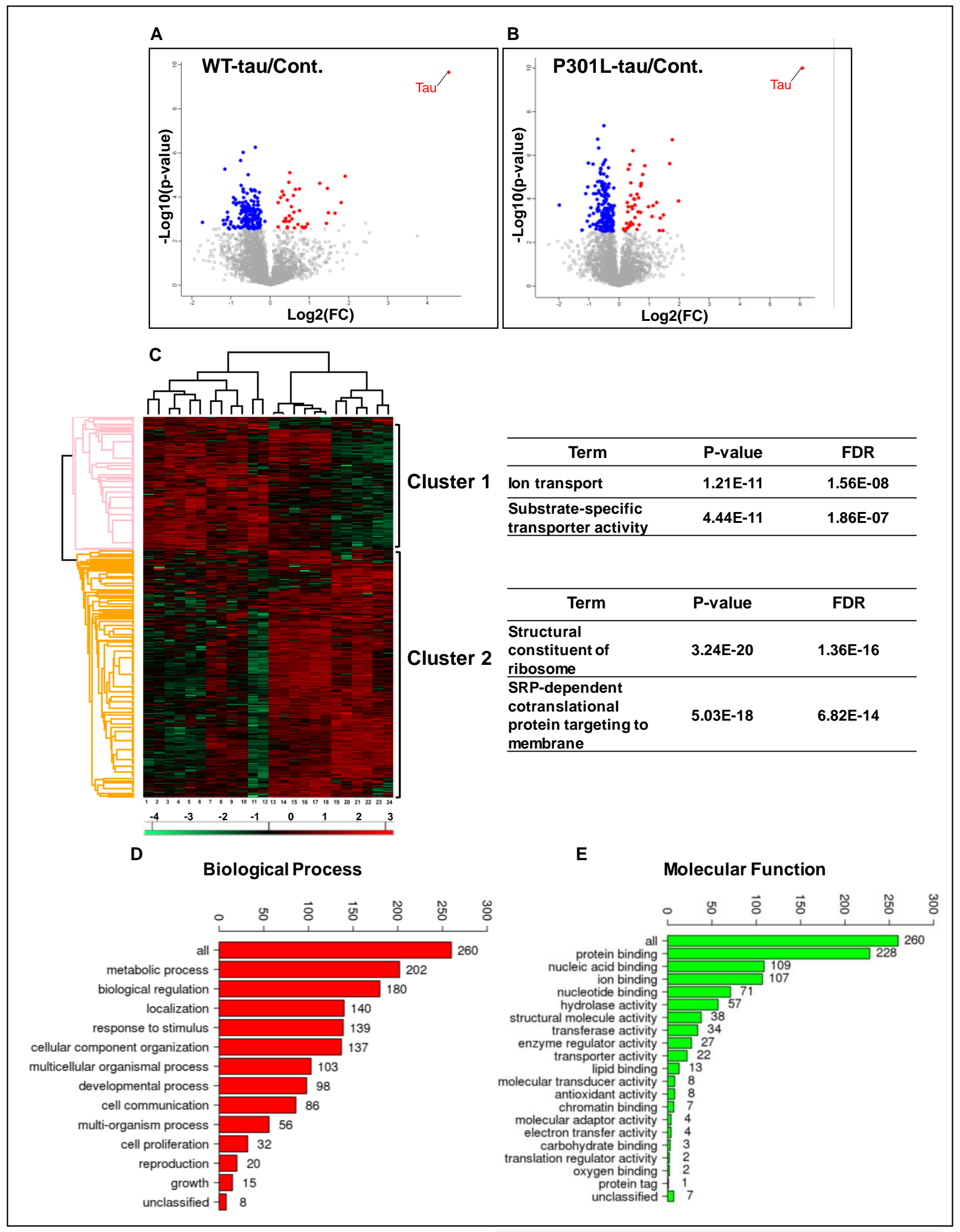

Figure 31: Human tau-expression-induced proteomic alterations: A and B) Volcano plots of pairwise comparisons showing differentially expressed proteins in both WT-tau- and P301L-tau-expressing cells in comparison to controls. C) The heatmap of two-dimensional hierarchical clustering analysis of 314 DEPs among technical and biological replicates generated by Perseus software (version 1.5.0.31). The $\log _{2}$-transformed expression values of significantly regulated proteins were normalized to the Z-score for all the replicates. The columns are representing samples (tau-expressing cells: lanes 1-12 and control: lanes 13-24) and rows indicating significantly modulated proteins (green, down-regulated: red, up-regulated). Functional enrichment analysis of DEPs re- 
sulted in multiple categories that were enriched in the two clusters. The enriched functional terms with their $p$ values and FDR values are shown on the right side (Fisher's exact test with FDR multiple test correction). The Fisher's exact test was used to select the significant GO terms identified by a p-value $<0.05$. D and E) GO-slim summary from WebGestalt, GO classification of proteins in both clusters. The number of proteins associated with each GO term in the "Biological Process" and "Molecular Function" domains is indicated in red and green bar charts, respectively.

Detailed analysis of the "Molecular Function" GO-terms of DEPs revealed RNAbinding (enrichment ratio $=3.58$ ) and structural constituent of ribosome (enrichment ratio $=7.3$ ) as the most enriched terms (Fig. 32).

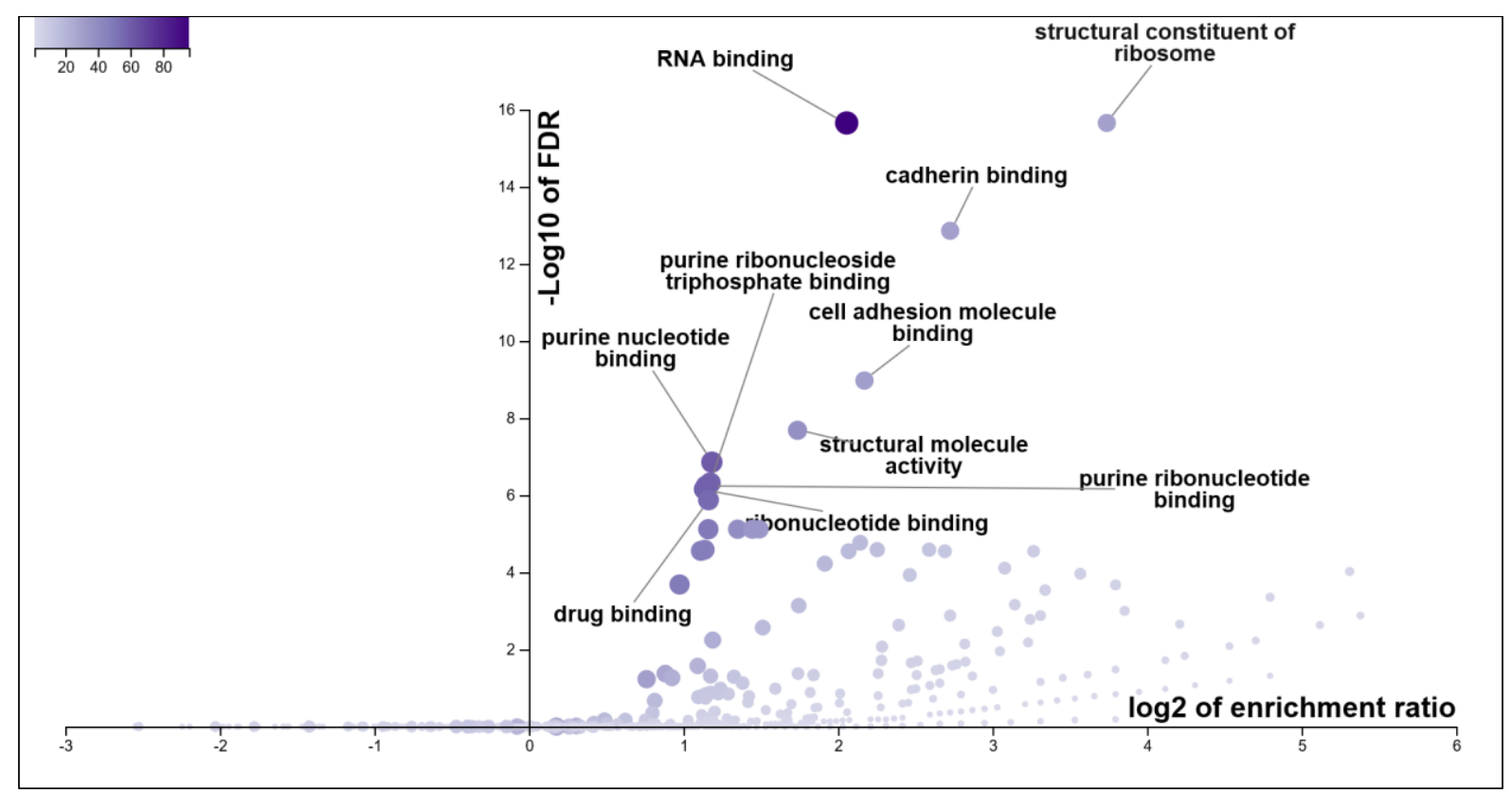

Figure 32: Volcano plot showing significantly enriched Molecular Functional terms from DEPs (314). The vertical axis is showing - $\log _{10}$-FDR value and $x$-axis represents the $\log _{2}$-transformed enrichment ratio obtained from WebGestalt.

\subsection{Canonical pathway analysis}

To find out major canonical pathways modulated after human tau expression, Ingenuity Pathway Analysis (IPA, Qiagen, USA) was performed. The proteomic candidates were sorted into 67 canonical pathways. According to their p-values, the top 5 significant pathways identified in WT-tau-expressing cells as compared with mocktransfected cells were eukaryotic initiation factor 2 (elF2) signalling ( $p$-value $=2.28 \mathrm{E}$ 23 ), regulation of elF4 and p70S6K signalling ( $p$-value $=6.44 \mathrm{E}-07$ ), mTOR signalling 
$(p$-value $=5.60 \mathrm{E}-06)$, mismatch repair for eukaryotes $(p$-value $=4.93 \mathrm{E}-05)$, and proline biosynthesis I ( $p$-value $=1.23 \mathrm{E}-04)$.

The most significant canonical pathways modulated after P301L-tau expression were EIF2 signalling ( $p$-value $=3.23 \mathrm{E}-18)$, interferon signalling ( $p$-value $=2.86 \mathrm{E}-04$ ), mTOR signalling ( $p$-value $=9.50 E-04)$, DNA double-strand break repair by nonhomologous end joining ( $p$-value $=1.11 \mathrm{E}-03$ ), and telomere extension by telomerase $(p$-value $=1.28 \mathrm{E}-03)$. Overall, EIF2 signalling was the most significant of the enriched canonical pathways.

\subsection{Disease- and function-based protein networks}

In addition to pathway mapping, DEPs were also categorized into related diseases and functions. The most significant networks associated with significantly modulated proteins are listed in annexure data for both WT-tau- and P301L-tau-expressing cells, respectively (Tables 18 and 19). The most significant network that was also shared between WT- and P301L-tau-expressing cells was "RNA damage and repair, protein synthesis" (score $=61$ ). Among these networks, two most significant networks are described below.

\subsection{Protein network associated with RNA damage and repair, protein syn- thesis, cancer}

The most enriched network that was common between both types of tau-expressing cells "RNA metabolism and protein synthesis" was composed of 27 focus molecules, including 60S ribosomal subunit, C7orf50, CDK4/6, Eif4g, ERK1/2, IFIT1, importin beta40s subunit, RPL15, RPL18, RPL18A, RPL19, RPL23A, RPL27A, RPL31, RPL32, RPL36, and RPL37A, among others. Biological Processes associated with this network were visualized by Cytoscape (3.6.1) (Fig. 33). Significant Biological Processes associated with this network include large and small ribosomal subunit, RNP complexes, SRP-dependent co-translational protein targeting to membranes, among other processes (Fig. 33). 


\section{Results}

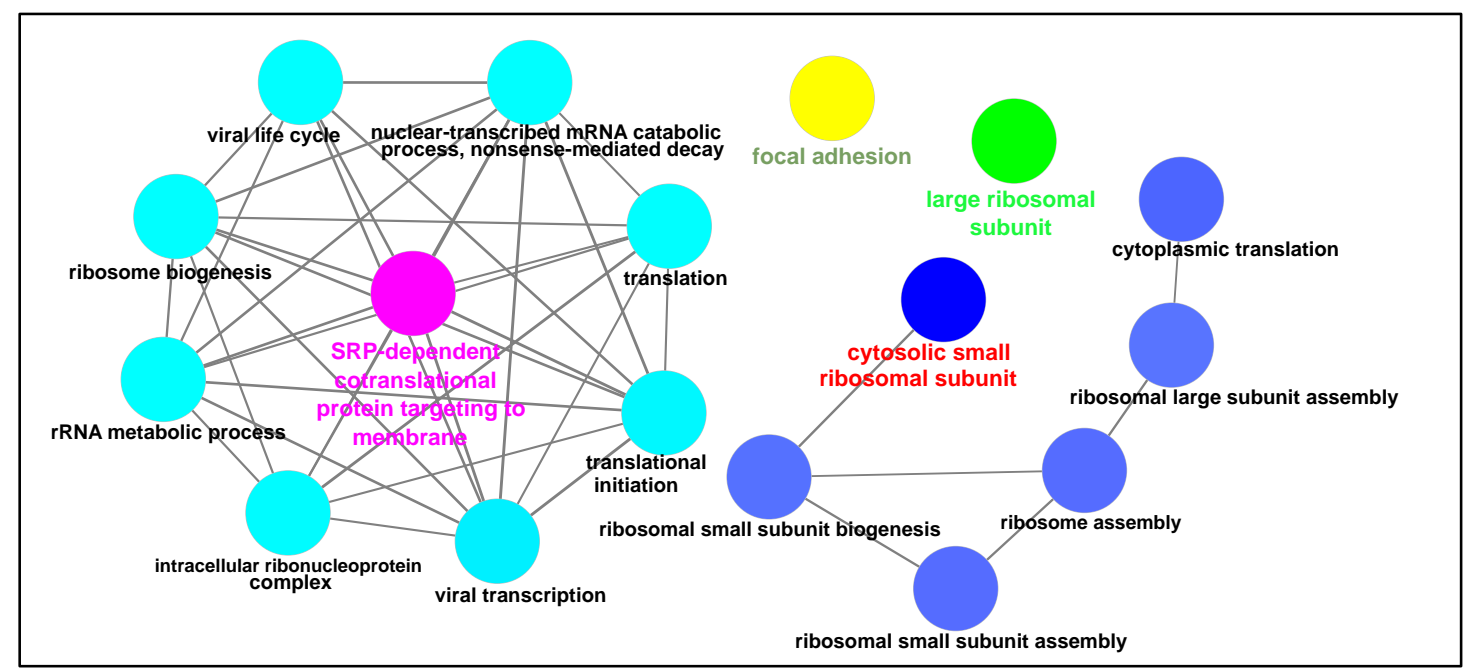

Figure 33: RNA damage and repair, protein synthesis, cancer. The Biological Processes associated with this IPA-based network were visualized by Cytoscape (3.6.1). The Cytoscape plugin ClueGO was used to identify enriched Biological Processes from the network molecules. Nodes with related Biological Processes are marked with the same colour.

\subsection{Protein network associated to cell morphology, cellular assembly and organization, DNA replication, recombination, and repair}

The second most enriched network was "cell morphology, cellular assembly and organization, DNA replication, recombination, and repair network", consisting of 20 focus molecules from DEPs from proteomic dataset. This complex network was interconnected with Biological Processes associated to homeostatic processes, including telomere maintenance and organization, DNA metabolic processes and chromosome organization, among others (Fig. 34).

Proteomics investigation with functional characterization has provided a comprehensive overview of major alterations associated with tau toxicity and downregulation of SFPQ. Analysis of global proteomic alterations revealed two major themes "RNA metabolism and protein synthesis", and "DNA homeostasis-related processes", that were significantly altered after human tau expression. 


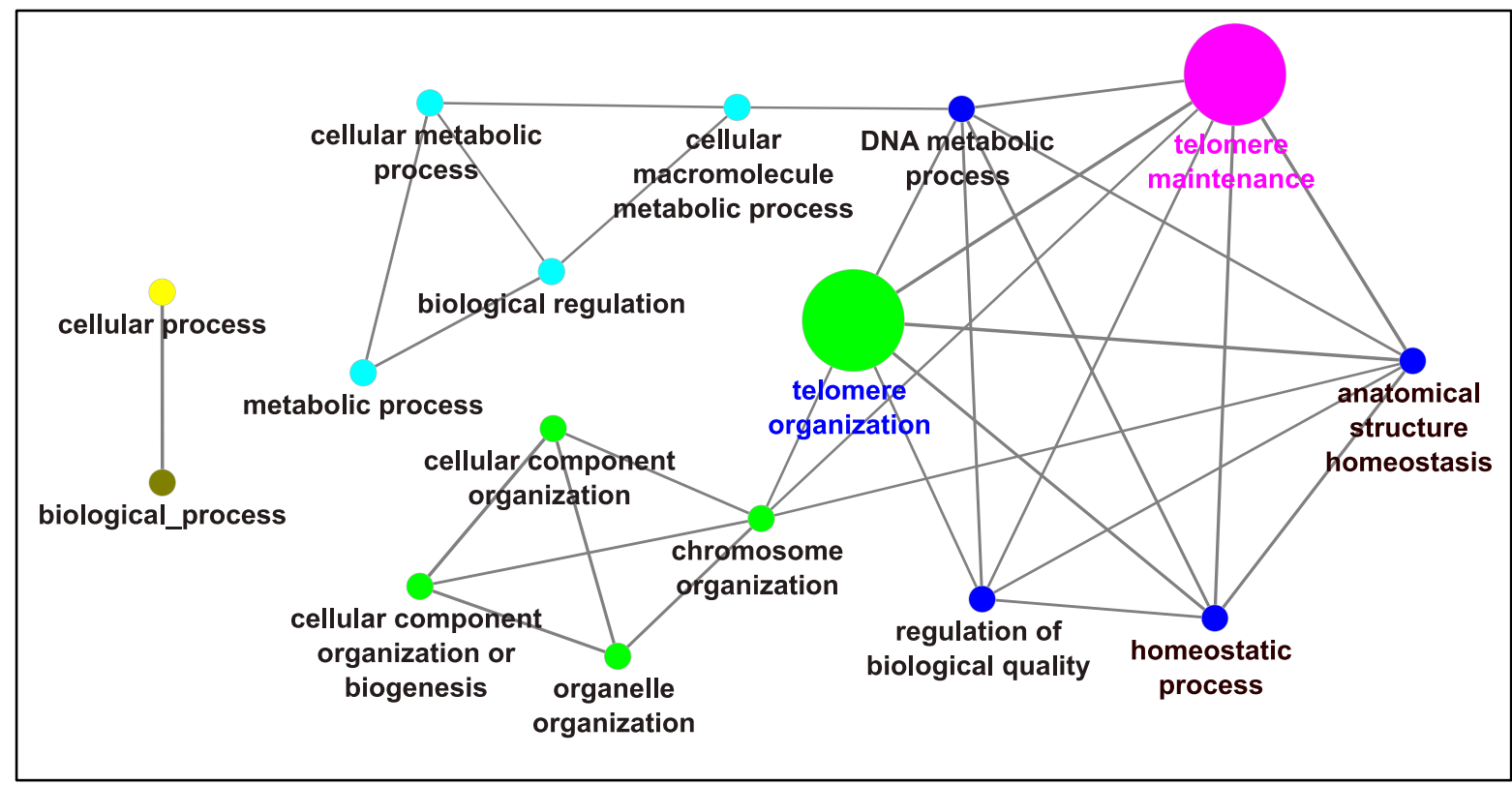

Figure 34: Biological Processes associated with most enriched network molecules from IPA-based network "Cell morphology, cellular assembly and organization, DNA replication, recombination, and repair". The final network was visualized using Cytoscape (3.6.1). The Cytoscape plugin ClueGO was used to visualize Biological Processes from the most enriched network molecules. The nodes from related functional terms are of the same color. The size of the node is corresponding to $\mathrm{BH}$-corrected $\mathrm{p}$-value and width of lines is representing extent of the overlap between related terms.

\subsection{Translation of SFPQ-tau-TIA-1 in the 3xTg-AD mice model}

Given the long incubation periods of clinically silent neurodegeneration and the manifestation of $A D$ at later stages, knowledge of the modifiable risk factors at presymptomatic stages of the disease is crucial. To extend our results from the analysis of terminal stage pathology from the human brain, a time-dependent expression profile of proteomic signatures was examined in a mouse model of $A D$, termed $3 \times \mathrm{Tg}$ $A D$, both at pre-symptomatic and symptomatic stages of the disease.

Mice were injected with $10 \%$ brain homogenate from AD patients. Animals were sacrificed at four time points spanning from early and late pre-symptomatic to early symptomatic and late symptomatic stages, e.g. at 3-, 6-, 9- and 12-months post inoculation (mpi). Total protein expression levels between inoculated and noninoculated control mice were determined at each time point. In summary, we found that protein expression during disease progression was dynamic and characterized by distinct changes in the global expression levels. 


\subsubsection{Differential expression of tau in AD mice}

Hyperphosphoylation of tau protein and concomitant formation of NFTs is a major hallmark of $A D$ and closely correlates to cognitive loss. Therefore, tau phosphorylation status was investigated in the mice model at all stages of the disease using immunoblotting analysis. The level of total tau was significantly decreased at the terminal stage between the experimental and control groups ( $<0.05)$ (Fig. 35A and B). There was a trend of increased phospho-tau levels at the early pre-symptomatic stage in experimental animals, when compared to controls, with no significant changes observed at later stages (Fig. 35A and C).

\subsubsection{Dysregulation of SFPQ at early and late-symptomatic disease stag- es}

Interestingly, levels of SFPQ were significantly altered at early pre-symptomatic phase with a significant increase in comparison to controls (Fig. 36A and D). At middle stages of the disease, expression levels returned to basal level and underwent a direct reversal at late-symptomatic stage of the disease with a drastic reduction at 12 mpi (Fig. 36A and D). Within the sensitivity of the Western blot, the band for SFPQ disappeared completely at the late-symptomatic stage of the disease, which coincided well with the massive reduction of SFPQ at the terminal stage of the disease observed in the postmortem brain, particularly in the rapidly progressive forms of dementia (rpAD and sCJD). 


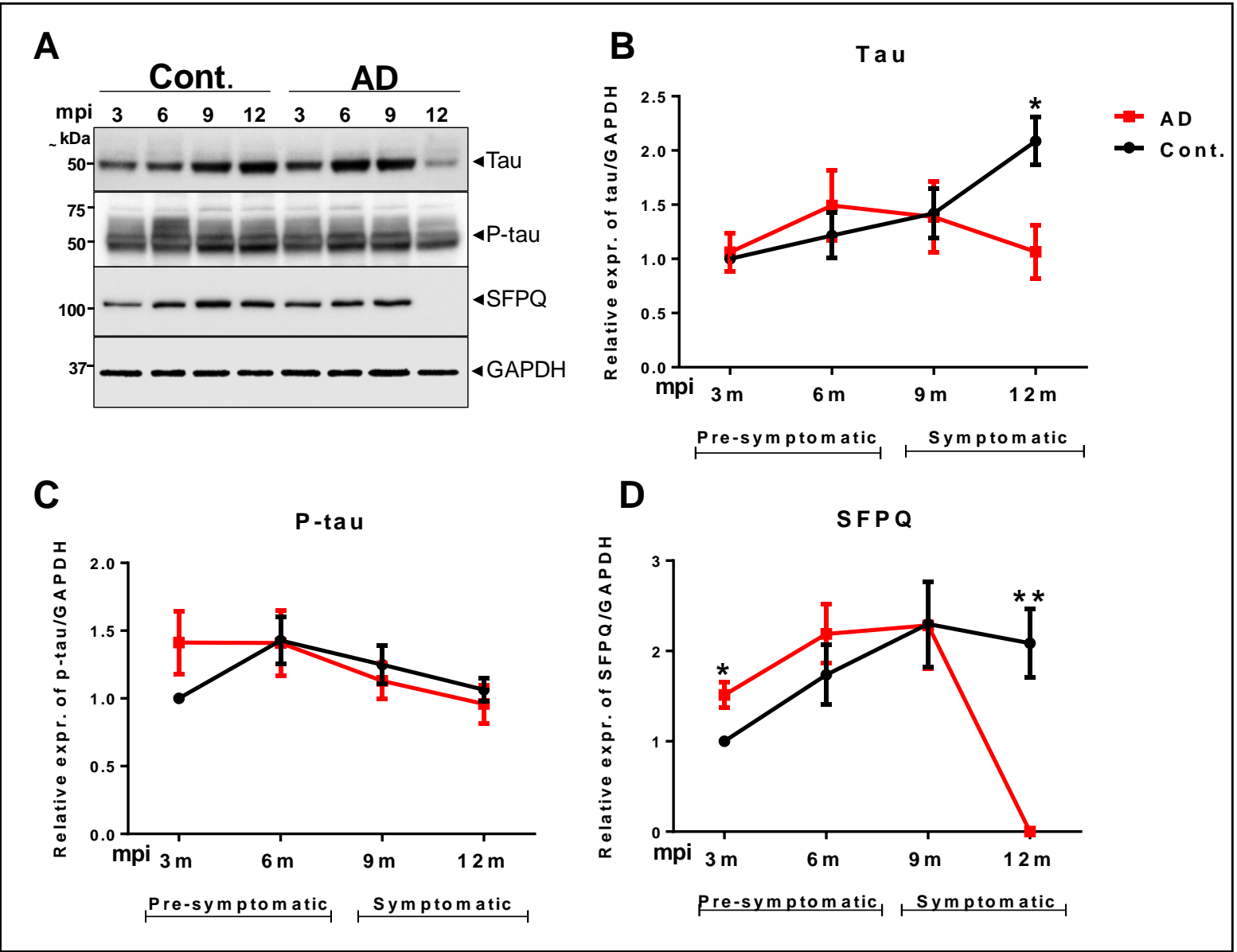

Figure 35: Temporal expression profile of tau, phospho-tau and SFPQ. A) Representative immunoblot images showing expression of tau, phospho-tau and SFPQ from AD $(n=4)$ mouse brain cortical tissues at the indicated ages (mpi: months post inoculation) and respective controls $(n=4)$. The GAPDH was used as a loading control. B-D) The densitometric analyses from three independent experiments were performed with Image Lab software. Unpaired $t$-test was performed to calculate statistical significance at each time point. ${ }^{*} p<0.05,{ }^{* *} p<$ $0.01,{ }^{* *} \mathrm{p}<0.001$.

\subsubsection{Alterations in TIA-1 levels at early pre-symptomatic and terminal stage of the disease}

Furthermore, we also studied the expression profile of TIA-1 in the AD mouse model at different time points. Two antibodies were used to detect both the $\mathrm{C}$ - and $\mathrm{N}$ terminus of TIA-1 (TIA-1 abcam: recognizing amino acids 350 at the C-terminus; TIA-1 sc-166247 recognizing amino acids 37-65 at the N-terminus). The protein levels of TIA-1 were significantly decreased at terminal stages of the disease (12 mpi) for both antibodies (Fig. 36A, B and C). For TIA-1 (recognizing amino acids 350 to the C-terminus), a significant increase was observed at the early pre-symptomatic 
stage of the disease, which then returned to basal levels at the middle stages of the disease (Fig. 36A and B). For C-terminal specific antibody of TIA-1, a low molecular weight band at the terminal stage $(12 \mathrm{mpi})$ was observed, which seems to be a cleavage product of TIA-1 (Fig. 36A, denoted with star*). The levels of VCP were also assessed at both pre-symptomatic and symptomatic stages in 3xTg-AD mice model. An increase in VCP levels was observed at the terminal stage of the disease, although that was found not to be significant (Fig. 36A and D).

In summary, the time-dependent expression profile with prominent changes in SFPQ, tau and TIA-1 at early pre-symptomatic stages suggested an altered regulation of these molecules at the very early stages of the disease.

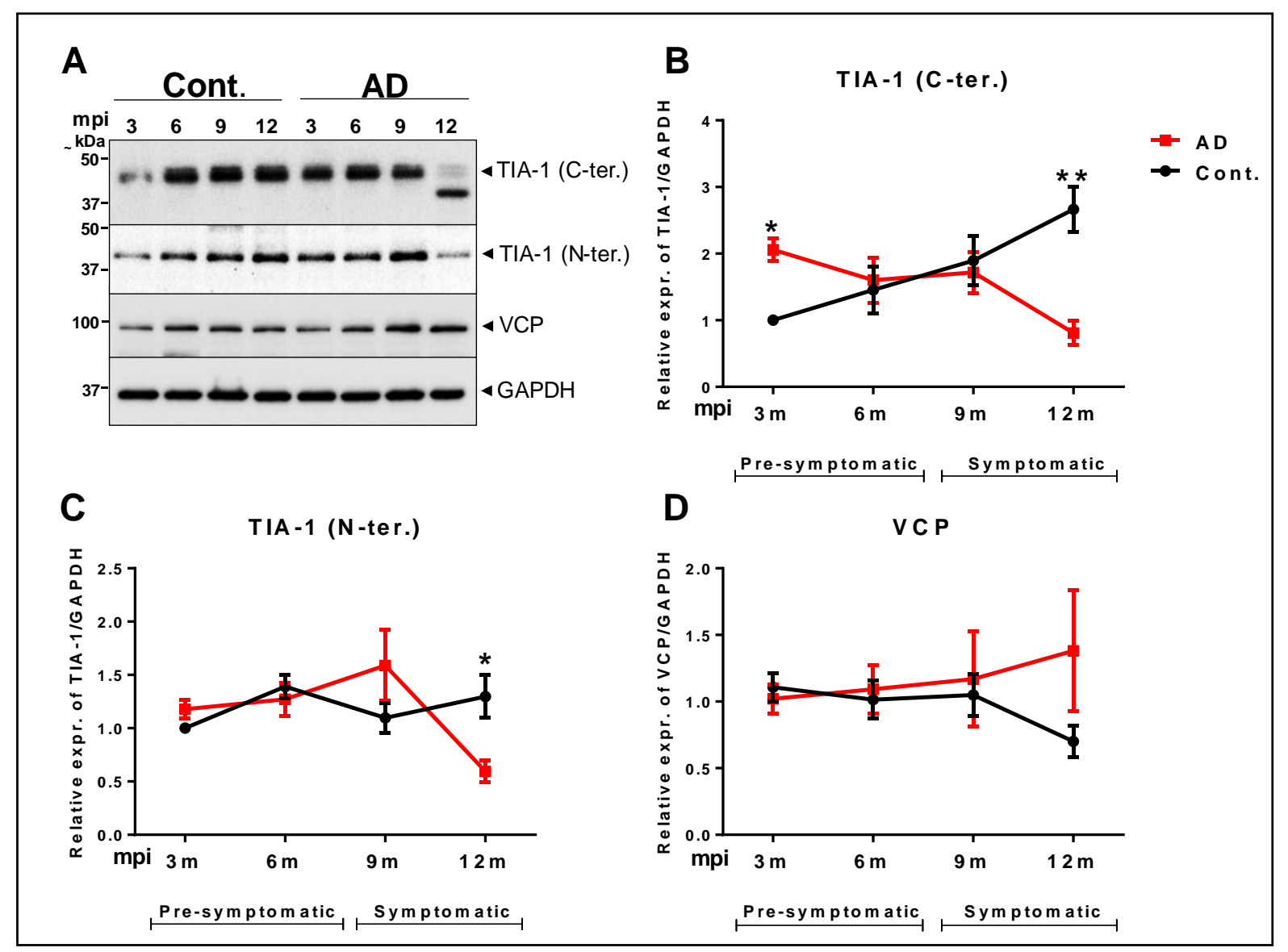

Figure 36: Alterations in TIA-1 levels during disease progression. A) Representative immunoblot images showing expression of TIA-1 (C- and N-terminal) and VCP from AD-like $(n=4)$ mouse brain cortical tissues at the indicated ages (mpi: months post inoculation) and respective controls $(n=4)$. $\left({ }^{\star}\right)$ Band may indicate proteolytical degradation of the C-terminus. The GAPDH was used as a loading control. B-D) Unpaired $t$-test was used to calculate significance. ${ }^{*} \mathrm{p}<0.05,{ }^{* *} \mathrm{p}<0.01$. 


\section{Discussion}

Dysfunctional regulation of RNA-binding proteins is a characteristic feature of many neurodegenerative diseases. Although precise mechanisms are still not clear, but it is increasingly evidenced that RBP anomalies are linked to neurodegenerative processes and/or accelerating their progression. To this end, the present study identified and characterised the RNA-binding proteome from human brain frontal cortex of three neurodegenerative entities, namely spAD, rpAD, and SCJD (MM1 and VV2 subtypes), as well as control subjects, using a brain-derived RNA-based pull-down approach followed by mass spectrometry analysis. Cortical region was studied due to maximum pathological manifestations in this region at end stages of the disease (Braak and Braak, 1991). Two mass spectrometry approaches (LFQ-MS and SWATH-MS) were employed in the present study, to gain a deeper insight into the RNA-binding proteome. Proteomic investigation revealed characteristic quantitative and qualitative changes in the identified RNA-binding proteome in a disease subtype-specific manner.

\subsection{RBPome alterations in neurodegenerative diseases}

Interestingly, global enrichment profile of RNA-binding proteomic candidates from the LFQ-MS analysis demonstrated major similarities between rpAD and SCJD, compared with spAD (Fig.12B). Although rpAD has same core pathological features ( $A \beta$ and tau tangles) in common with spAD based on RBPome signatures, rpAD displayed more similarity with SCJD group in comparison with spAD. These findings suggest that, both forms of rapidly progressive dementias (rpAD and SCJD) have similarities in RBP-mediated neuropathogenesis at the terminal stage of the disease. Furthermore, these similarities also argue that these RBP-related processes may have a pathophysiological role in the rapid progression of these diseases.

The differential enrichment analysis of identified RNA-binding proteome revealed distinct proteomic clusters specific to each group (Fig. 12B). Overall, specific enrichment of these distinct protein clusters may indicate differences in the RBP-RNA interactions in response to disease stress. These specific sets of proteins can be of potential relevance to identify subtype-specific differences of these heterogenous 
diseases. Furthermore, the identification of RNA-binding proteome opens many new therapeutic targets for exploration (Table 15).

As the RBPome changes were more prominent in the rpAD and sCJD groups, we employed quantitative SWATH-MS technology to get a deeper insight into the RBPome of these rapidly progressive forms of dementia. This finding also served as a confirmatory approach to the LFQ-mass spectrometry. It is notable that the RBPome from SWATH-MS virtually recapitulated the LFQ-based proteomic data. In consistence with deep proteome coverage by SWATH-MS, this analysis indicated 477 proteins which showed differences in the abundance between rpAD and two subtypes of SCJD MM1 and VV2. One interesting finding evident from the hierarchical clustering analysis of differentially abundant proteins was the similarity between rpAD and SCJD-MM1 subtype compared with the profile from SCJD-VV2 subtype. Both the rPAD and SCJD-MM1-subtype were segregated into a single cluster, while SCJD-VV2 subtype segregated into a separate cluster. The methionine homozygosity (M/M) at codon 129 of the prion protein gene (PRNP) has been described as a risk factor for AD (Gacia et al., 2006; Schmidt et al., 2010). Similarities between the rPAD and SCJD-MM1 subtype observed in the current study support the notion that methionine homozygosity is not only a risk factor for $A D$, but it may have a role in the rapid progression of the disease.

\subsubsection{Functional analysis of MS results}

Functional enrichment analysis of the identified RBPome, highlighted major changes related to RNA metabolism, stress response, metabolic processes, and immune system among others. The enrichment of metabolic proteins in the current RBPome dataset demonstrates an interaction between RNA-related processes and metabolic changes during the disease. The regulation of metabolic processes resulting from disease stress is necessary to facilitate survival of the neurons. The RBPs may be engaged in regulatory plasticity, which is required for the instant stress response. In addition, the identification of immune-system-related proteins, supports the integral role of inflammatory processes in the pathogenesis of these neurodegenerative diseases. Finally, our data is also consistent with the well-known functions of RBPs in the post-transcriptional stress response and RNA metabolism. In summary, our findings denote that changes in the RBPs, in addition to metabolic and inflammatory re- 


\section{Discussion}

sponses, are an integral part of the pathological features of these neurodegenerative diseases.

Furthermore, the Biological Process related to "Localization" including protein and macromolecular localization was a leading term enriched in rapidly progressive $A D$, indicating disturbances in the protein localization as a pathomechanism in rpAD. Our study identified numerous important proteins, e.g. importin subunit-beta1 (nuclear factor p97), clathrin coat assembly protein AP180 or secretogranin, which were uniquely present in the rPAD dataset. These signatures can be of potential relevance to distinguish the rapid variant of $A D$ from both normal and classical $A D$-related dementia. Some of these proteins might represent novel biomarkers whose expression, aggregation or posttranslational modifications might reflect distinct elements of the disease process.

\subsubsection{Proteins involved in RNA metabolism and stress response}

The functional categories related to stress response, antioxidant activity, and RNAbinding were specifically enriched in rpAD and SCJD groups, compared with control and SpAD groups, indicating a more aggressive dysregulation of RBP-related processes in these fast-progressive forms of dementia. Stress in almost every form has been linked to fast progression in not only $A D$ but also other neurodegenerative diseases, e.g. Parkinson's and Huntington's diseases (Hiller et al., 2017). Studies from many groups and different animal models have reported that stress is a factor leading to fast progression of $A D$, including increased plaque assembly, hyperphosphorylation of tau, and tangle formation. Not only in animal models, but many studies in humans have elaborated that exposure to extreme stress could also lead to rapid progression of sporadic AD (Herbert and Lucassen, 2016; Mejía et al., 2003), and earlier onset in familial AD (Mejía et al., 2003). Data showing the role of stress response in prion diseases have also been reported (Goggin et al., 2008; Mays et al., 2019).

In summary, data from this study and reports from the literature highlight a significant role of RBPs through stress response in the rapid progression of the disease. Recently, alteration of tau protein has also been linked to stress response in $A D$ (Brunello et al., 2016; Vanderweyde et al., 2016), further strengthening the hypothe- 


\section{Discussion}

sis that stress and post-transcriptional regulatory processes associated with RBPs are an integral feature of these neurodegenerative disorders.

\subsubsection{Canonical and putative RNA-binding candidates}

The present study identified many putative RNA-binding protein candidates (e.g. metabolic and catalytic enzymes) in addition to canonical RBPs, in agreement with previous reports (Shchepachev et al., 2019). The identification of these putative RBPs in the RNA-binding proteome data points towards a cross-talk between RBPs and other proteins (e.g. metabolic enzymes) to meet the everchanging microenvironment surrounding the neuron. Dysregulation of these intricate networks of proteins in the neurons may start a cascade of aberrant signaling, eventually leading to neurodegeneration.

Many studies identifying RNA-binding proteomes have reported a large number of putative RBP candidates, that have no prior linkage to RNA-related functions (Baltz et al., 2012; Beckmann et al., 2015; Castello et al., 2012; Castello et al., 2013). These putative RBPs, which were categorized as enigmRBPs, have many diverse roles in cellular homeostasis, including actin remodeling, protein folding, and many metabolic enzymatic activities; however the functions of most of these enigmRBPs are largely unknown. To date, the role of only a few enigmRBPs, e.g. metabolic enzyme (IRP1), has been discovered (Castello et al., 2015; Hentze et al., 2018). It is notable that some of the identified putative RBP candidates (e.g. SNG3, HEBP1, SV2A) may not have RNA-binding activity themselves. They were identified in the present study due to binding with other proteins that do have RNA-binding activity.

These putative RBP candidates can potentially be beneficial for the cell. Interactions between RNA and these putative RBPs (specifically metabolic enzymes) can be used for spatial sorting of related enzymes. This spatial sorting helps the cell to boost the metabolic flux (Castello et al., 2015).

\subsubsection{Prion-like-domain (PLD)-containing proteins}

Prion-like-domain-containing proteins can be both beneficial and harmful for the cell (Sabate et al., 2015a; Sabate et al., 2015b). In the current study, twenty-four PLDcontaining proteins, fulfilling the requirements to potentially behave as prion-like pro- 
teins were identified (e.g. BSN, SFPQ, EWS, PRIO). Prion-like domains are essential for RBP functions and enable them to undergo liquid-liquid phase separation, that is the basis of the formation of higher-order structures, including oligomers and granules (Boeynaems et al., 2018; Riback et al., 2017).

In the present study, we observed that the PLD-containing protein SFPQ is exclusively enriched in the RBPome of rpAD and SCJD, suggesting a potential role of SFPQ in rapidly progressive dementias. Furthermore, SFPQ exhibited a considerably high score for prion-like domain (PLD-score $=28$ ), which is a very crucial factor contributing to pathophysiological functions of PLD-containing proteins. Indeed, we discovered dysregulation of SFPQ in association with tau and TIA-1 proteins, particularly in the postmortem brains from rpAD and SCJD patients.

\subsection{Pathological characterization of SFPQ in the human brain}

\subsubsection{SFPQ dysregulation in the rpAD and SCJD brains}

Emerging evidence supports that neurodegenerative anomalies modulate the expression of RBPs (Conlon and Manley, 2017). Interestingly, we identified a significant reduction in SFPQ at the protein level in the frontal cortical region of rpAD and the subtypes of SCJD MM1 and VV2. A trend was also observed for SpAD cases, although this was not significant. Ke et al. (2012) reported a significant reduction in SFPQ levels with advanced Braak stages in the entorhinal cortex of AD patients. Specific reduction of SFPQ in both forms of rapidly progressive dementias (rpAD and SCJD) further implies that SFPQ may be involved in the rapid progression of these neurodegenerative diseases. To the best of our knowledge, this is the first study demonstrating dysregulation of SFPQ at both protein and mRNA level in the frontal cortex of specifically rpAD subjects.

The reduction of SFPQ has been linked to behavioral anomalies in mice, neuronal loss, and phospho-tau accumulation (Ishigaki et al., 2017). In another study, loss of SFPQ was found to lead to apoptosis in zebra fish, linking downregulation of SFPQ to neuronal cell death (Lowery et al., 2007). Splicing factor proline and glutamine rich is a predominantly nuclear protein involved in multiple functions in the neurons, including transcription, alternative splicing, DNA damage and repair, and transport of mRNAs through long axons (Knott, et al., 2016; Yarosh et al., 2015). It can be con- 
cluded that downregulation of SFPQ can contribute to neurodegeneration by affecting multiple functions of SFPQ, specifically in the nucleus.

At the mRNA level, SFPQ expression was elevated in rpAD, in contrast to the reduction at protein level. One plausible explanation for this observation could be that transcription of SFPQ is increased in order to compensate for loss of SFPQ at the protein level. The increased mRNA levels of SFPQ could also contribute directly to neurodegeneration. The excessive mRNA may sequester many proteins necessary for other cellular signalling, as has been noted for some other proteins involved in neurodegenerative disease (Greco et al., 2006; Sellier et al., 2014; Tassone et al., 2004). These aberrant masses of mRNA and protein may convert into the inclusion bodies (Greco et al., 2006; Iwahashi et al., 2006).

\subsubsection{SFPQ dislocation in the brain of rpAD patients}

Cytoplasmic mislocalization of many nuclear factors has been defined as a pathomechanism in several neurological diseases (Barmada et al., 2010; Bishof et al., 2018; Neumann et al., 2006; Vance et al., 2013). Additionally, in the current study, a drastic nuclear depletion was detected for SFPQ in the frontal cortex of rpAD subjects. Previously, one study has reported a complete dislocation of SFPQ in the hippocampus of $A D$ patients (Ke et al., 2012). Specifically, the higher dislocation/depletion rate $(91 \%$ of cells) observed in rPAD cases in the current study as compared to $\operatorname{spAD}(51 \%)$ and controls $(43 \%)$ suggests an important role of SFPQ in the rapid progression of the disease. Lu et al. (2018) demonstrated a moderate dislocation of SFPQ in AD brains, which was consistent with the observations regarding spAD described in this study. Based on data from the current study, from the frontal cortex and literature reports (showing dislocation in hippocampus), it can be concluded that dislocation of SFPQ is an important feature of Alzheimer's pathology, and different brain regions have variable intensity of SFPQ dislocation. Increased dislocation in rpAD subjects may be due to higher cell death (through apoptosis), as cytoplasmic localization of SFPQ has been observed in apoptotic cells. The nuclear depletion of SFPQ, particularly in rpAD, may contribute to neurodegeneration by both the loss of nuclear functions (Lu et al., 2018) and toxic functions in the cytoplasm. 


\section{Discussion}

Nuclear loss of SFPQ could render cells more prone to DNA damage, considering that solid evidence has been established for a crucial role of SFPQ in the DNA double-strand break repair processes (Jaafar et al., 2017). Lu et al. (2018) reported a disrupted DNA organization in association with SFPQ dislocation. SFPQ depletion from the nucleus may induce cell death by contributing to mitosis, as a redistribution in the cytoplasm was observed in mitotic cells (Shav-Tal et al., 2001).

\subsubsection{SFPQ co-localization with the SG marker TIA-1 in the IPAD brain}

Nuclear depletion and dislocation of SFPQ was concomitantly associated with its cytoplasmic co-localization with TIA-1 (a classical marker of SGs) in rpAD subjects. A ring-shaped SFPQ was observed around the nuclei which co-localized with TIA-1. Previously, pathological SGs have been linked to mislocalization of tau, FUS and TDP-43 (Bosco et al., 2010; Liu-Yesucevitz et al., 2010; Vanderweyde et al., 2016; Yasuda et al., 2017).

Furthermore, SFPQ translocated from the nucleus and assembled into cytoplasmic TIA-1-positive stress granules in response to oxidative stress conditions in cultured cells. These findings from the current study indicate a role of SFPQ in the stress response under physiological conditions. The identification of SFPQ in the stress granule-interactome of U2OS cells (Jain et al., 2016) further confirms the involvement of SFPQ in the stress response. Pathological and persistent TIA-1-positive-stress granules have been implicated in AD (Apicco et al., 2018). Increased intron retention in SFPQ transcript has been reported in ALS patients (Luisier et al., 2018). Extensive binding of SFPQ to its retained introns leads to higher cytoplasmic abundance. This corroborates with our findings of increased mRNA levels of SFPQ in the rPAD brain, which might contribute to the altered localization observed in the rpAD cases.

Though predominantly a nuclear protein, multiple localizations of SFPQ have been reported. Phosphorylation at C-terminal tyrosines leads to accumulation at the nuclear envelope or in the cytoplasm (Lukong et al., 2009; Otto et al., 2001). This redistribution of SFPQ has been linked to cell cycle arrest (Lukong et al., 2009), apoptosis (Galietta et al., 2007; Shav-Tal et al., 2001), and splicing abnormalities (Heyd and Lynch, 2010; Melton et al., 2007). Furthermore, a role for SFPQ in the IRES (internal ribosome entry site)-mediated translation in the cytoplasm has also been reported 


\section{Discussion}

(Sharathchandra et al., 2012). From our results and literature reports, we propose three possible mechanisms responsible for the dislocation of SFPQ from the nucleus: (1) Chronic stress may turn physiological SGs into pathological, and hijack SFPQ in the cytoplasm. (2) Retained introns in combination with increased levels of SFPQ transcript may sequester SFPQ in the cytoplasm. (3) Redistribution of SFPQ into the cytoplasm is linked to cell death (apoptosis), specifically in rpAD cases.

\subsubsection{SFPQ and neurofibrillary tangles}

Co-aggregation of some splicing factors with tau protein in the cytoplasmic inclusions has been reported for both sporadic and familial AD cases (Bai et al., 2013; Bishof et al., 2018; Diner et al., 2014). The present study identified a depletion of both SFPQ and phospho-tau from the nucleus in brain tissue from rpAD patients. Both proteins showed cytoplasmic/perinuclear co-localization as compared to nuclear colocalization in control cases. One possible explanation for this observation could be that, at earlier stages, activated kinases can phosphorylate not only tau protein but also SFPQ. Cytoplasmic association of SFPQ with phospho-tau tangles, and their complete loss from the nucleus, suggests a change in the function of these proteins, extending the pathogenic role of tau to the nuclear processes.

Hernandez-Ortega et al. (2016) described a complete depletion of nuclear tau in the neurons bearing NFTs in hippocampal-CA1, entorhinal and temporal neocortical regions at the terminal stages of the disease. This altered localization of nuclear tau was associated with chromatin modifications (Hernandez-Ortega et al., 2016). In rpAD cases showing complete loss of nuclear tau, DNA-protective role of nuclear tau (Sultan et al., 2011) or heterochromatin stabilization function (Hernandez-Ortega et al., 2016; Sjöberg et al., 2006) will be disturbed. Furthermore, SFPQ has also been linked to telomere maintenance (Petti et al., 2019). Its dislocation from the nucleus along with tau protein may lead to impaired DNA functions and results in aberrant gene regulation. In summary, our data in corroboration with previous reports suggest the involvement of nuclear tau, possibly in conjunction with SFPQ, in the pathology of $r p A D$.

Interestingly, we detected a predominant nuclear localization for phospho-tau (S199) in the control subjects. Nuclear localization of phospho-tau has been reported in 
normal cell lines (Shea and Cressman, 1998), mouse brain (Lambert et al., 1995; Lu et al., 2013), and human brain (Brady et al., 1995). However, the significance of phospho-tau in the nucleus under control conditions is not clear. Localization of phospho-tau in the nucleus under control conditions, observed in the present study in combination with literature reports, highlights a potential role of tau in genome surveillance. Further knowledge on nuclear and, in particular, phosphorylated tau may provide clues in understanding pathological features of nuclear tau in neurodegenerative diseases.

In spAD subjects, tangled tau was even observed in the nucleus in agreement with previous findings (Fernandez-Nogales et al., 2017; Lu et al., 2018), where it colocalized with SFPQ. Identification of tangled tau in the nucleus extends pathological features of tau not only to axon or somatodendritic compartments but also to nuclear processes. Previously, rod-like deposits of tau were also identified in the nuclei of subjects with AD and Huntington's disease (Lu et al., 2014). Collectively, evidence from the current study in corroboration with previous studies suggests a role for tau within the nucleus under normal and disease conditions. Furthermore, dislocation/depletion of both SFPQ and phospho-tau, specifically in the rpAD cases, may be linked to distinct molecular pathways in subtypes of AD.

Biochemically, there were no significant differences detected for total tau in the current study in the postmortem brains. We were able to detect significant differences in the SDS-resistant HMW-tau ( 120KDa) between SpAD and control subjects. Interestingly, a trend for lower ratio of phospho-tau/tau was observed in rpAD cases as compared with SpAD patients, in line with a previous report (Ba et al., 2017). This decreased phospho-tau/tau ratio indicates a reduced rate of tau phosphorylation in rpAD subjects, contrary to higher phosphorylation, which is a cardinal feature of spAD pathology. This difference in the ratio of phospho-tau/tau may be of potential significance for the development of a progressive form of AD.

\subsubsection{SFPQ co-localizes with oligomeric tau in the rpAD}

Although cytoplasmic tau deposits are a burden for the cell, it is rather the toxic soluble oligomeric species of tau that are the real culprits associated with cognitive decline, neuronal dysfunction, and death (Guerrero-Muñoz et al., 2015; Shafiei et al., 2017). Interestingly, significant co-localization was detected for tau oligomers and 
SFPQ in the rpAD subjects. This interaction between SFPQ and tau oligomers has potential relevance for oligomerization, and subsequent misfolding of tau protein. Previously, co-aggregation of tau oligomers with TIA-1 has been reported in animal models of tauopathy (Apicco et al., 2018; Vanderweyde et al., 2016). In response to different kinds of stresses, SG formation brings together many intrinsically aggregated-prone proteins (e.g. TIA-1, tau, and SFPQ) to form reversible SGs. Chronic stress can turn these physiological SGs to insoluble and pathological SGs (Wolozin, 2012). Based on results from the current study, demonstrating

A) stress-induced redistribution of SFPQ into cytoplasmic TIA-1-positive SGs in cultured cells,

B) its nuclear depletion and co-localization with cytoplasmic TIA-1 in the human brain of rpAD subjects,

C) SFPQ co-localization with tau oligomers and tangles in the rpAD brains,

D) its high score for PLD (PLD score $=28$ ), and

E) its LLPS property (catGRANULES score $=1.66$ ),

it is reasonable to propose that SFPQ is an important component of AD pathology, particularly of rapidly progressive AD.

Our findings suggest that dysregulated SFPQ can interact with (hyperphosphorylated) tau and its oligomeric form in stress granules, providing an intimate link between SFPQ, tau pathology and SGs. Dislocation of SFPQ may lead to impaired nuclear functions of SFPQ, e.g. DNA damage and repair, telomere stability, and splicing abnormalities. Toxic gain of function in the cytoplasm may contribute to aberrant dynamics of SGs, oligomerization, and misfolding of tau protein (Fig. 37). Based on results from the current study, we could state that SFPQ pathology is linked with tau protein, more robustly in rapid progressive form of $A D$. These results provide a new insight into the relationship between SFPQ and tau pathological features in rpAD. 


\section{Discussion}

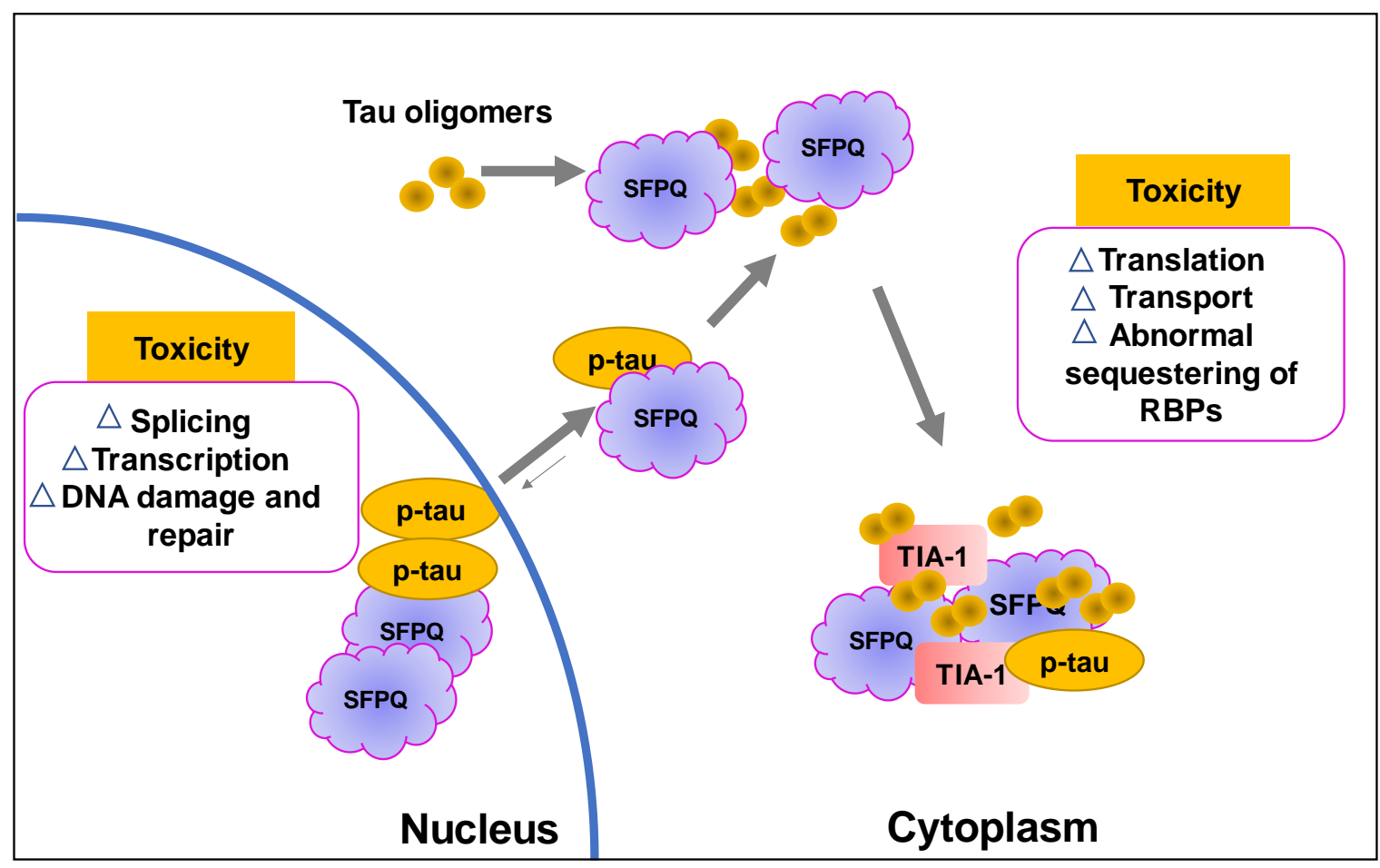

Figure 37: Pathological characteristics of SFPQ, phospho-tau and TIA-1 in the human brain. This figure describes pathological features observed for SFPQ, TIA-1 and phospho-tau in the postmortem brains of rpAD cases. The RNA-binding protein SFPQ is normally localized predominantly in the nucleus, where it performs multiple functions including splicing, transcription, and DNA damage and repair. In the control subjects, significant co-localization was observed for SFPQ and phospho-tau (S199) in the nucleus (1). In rpAD subjects, a complete nuclear depletion and cytoplasmic co-localization was observed for SFPQ and tau tangles (2), and tau oligomers (3). Furthermore, SFPQ co-localization with TIA-1 was identified significantly in brain samples from rpAD patients (4). At protein level, SFPQ was significantly downregulated, and TIA-1 was upregulated in rpAD group, in comparison to spAD group. Our findings suggest that dysregulated SFPQ can interact with (hyperphosphorylated) tau and its oligomeric form in stress granules, providing an intimate link between SFPQ, tau pathology and SGs. Overall, dysregulation of SFPQ in the form of reduction and nuclear depletion/dislocation can affect the cell biology by two-ways.1) Dislocation of SFPQ may lead to impairment of nuclear functions of SFPQ e.g. DNA damage and repair, telomere stability, and splicing abnormalities. 2) Toxic gain of function in the cytoplasm may contribute to aberrant dynamics of SGs as well as oligomerization and misfolding of tau protein.

\subsubsection{Differential regulation of SG marker TIA-1 in disease-subtype-specific manner}

Previously, TIA-1 has been linked to tau misfolding (Vanderweyde et al., 2016) but the expression of TIA-1 has not been investigated in the various $A D$ entities. In the present study, we detected a significant reduction in TIA-1 levels in SPAD and SCJDsubtypes. It was shown that TIA1 reduction (haploinsufficiency) is cytoprotective in vivo, leading to increased survival in a mouse model of tauopathy due to reduced neurodegeneration and improvement in cognitive deficits (Apicco et al., 2018). A sig- 
nificant increase in TIA-1 levels in $r P A D$ as compared to SPAD may contribute to higher neurodegeneration in rPAD cases.

Our data show that valosin-containing protein is specifically enriched in the RNAbinding proteome from rPAD and SCJD subjects, suggesting a possible involvement of VCP in these diseases. In our study, a significant increase of mRNA coding for VCP was detected in rpAD subjects. This protein plays an important role in the clearance of SGs (Turakhiya et al., 2018). Malfunction of VCP due to mutations has been associated with increased build-up of stress granules in other neurodegenerative diseases, e.g. ALS and fronto-temporal dementia (FTD) (Ramaswami et al., 2013; Wolozin, 2012). Elevated expression of VCP at the mRNA level may have a role in the formation and clearance of neuronal inclusions in various neurodegenerative diseases (Mori et al., 2013). It can be concluded that VCP dysregulation is a common pathological feature of several neurodegenerative diseases including rpAD.

\subsection{Translational aspects of SFPQ in cellular models}

\subsubsection{Cellular model of stress}

To understand the role of target proteomic candidates for SG biology, HeLa cells were used as an appropriate model for stress induction (Aulas and Vande Velde, 2015). These cells offer numerous technical advantages, as they are easy to maintain in culture and reliably result in high transfection efficiency (Bali et al., 2012). Though non-neuronal, this cell line was used for the current study because it produced prominent SGs. Compared to other cell lines, the increased size of HeLa cells allows the accurate distinction of cytoplasmic SGs.

\subsubsection{SFPQ recruitment into SGs after oxidative stress treatment}

In this study, we demonstrate that SFPQ is recruited into SGs that are induced in response to treatment with sodium arsenite. The SFPQ protein is an important component of RNA transport granules in dendrites (Kanai et al., 2004; Kunde et al., 2011; Zhu et al., 2005). However, it is not known whether SFPQ plays a role under stressful conditions. In the current study, it was found that endogenous SFPQ colocalizes with the core SG marker TIA-1 following exposure to oxidative stress. SFPQ is localized predominantly in the nucleus, but treatment with sodium arsenite 


\section{Discussion}

induced the redistribution of this protein into the cytoplasm. However, the quantity of translocated protein was small relative to the total amount of the protein. This redistribution led to the formation of cytoplasmic inclusions, which co-localized with SG marker, indicating that these inclusions are SGs. Association of SFPQ with SGs can be mediated through protein-protein or RNA-protein interactions. Ke et al. (2012) reported a redistribution and cytoplasmic accumulation of SFPQ in the form of vesicular aggregates in SH-SY5Y cells after overexpression of tau. Cytoplasmic redistribution in the form of inclusions has also been observed in N2a cells after SFPQ overexpression (Lu et al., 2018). Both of these studies reported the formation of cytoplasmic inclusions of SFPQ, but the nature of these inclusions is not known. Colocalization of SFPQ with TIA-1-positive stress granules, as shown in the present study, indicates that these are most likely SGs.

In summary, our results highlight a role of oxidative stress in the cytoplasmic redistribution of SFPQ and its incorporation into SGs. Furthermore, we also found a significant increase in SFPQ intensity levels after stress induction. It was reported that SFPQ sensitizes neurons to excitotoxic damage in vitro (Xu et al., 2005). Our findings indicate an important function of SFPQ under stressful conditions, allowing significant flexibility in gene regulation, therby enabling the cell to adjust in response to different environmental conditions. Based on our findings demonstrating oxidativestress-mediated redistribution of SFPQ into TIA-1-positive cytoplasmic SGs, it can be concluded that chronic stress may lead to nuclear depletion of SFPQ, which was observed in the postmortem brains of rpAD cases.

\subsubsection{Tau, TIA-1 and SFPQ in stress granules}

In our model of stress induction, we observed a co-localization of tau and phosphotau with TIA-1. Interestingly, for phospho-tau, we observed both nuclear and cytoplasmic localization with predominant nuclear reactivity, which was increased in response to stress treatment. Although tau is considered predominantly a cytosolenriched protein, several studies have reported its nuclear localization in both neuronal (Siano et al., 2019, Ulrich et al., 2018; Wang et al., 1993) and non-neuronal cell lines, including HeLa cells (Sjöberg et al., 2006). Higher immunoreactivity for phospho-tau in the nucleus during stress exposure may indicate a role of tau in cytoprotective mechanisms. Previously, a role of nuclear tau has been implicated in the 
compensatory mechanisms of the cell through epigenetic changes upon stress exposure (Frost et al., 2014; Mastroeni et al., 2011; Sanchez-Mut and Graff, 2015; Sultan et al., 2011). Notably, we found co-localization of SFPQ with tau and phosphotau in the granules after stress exposure. This co-localization of SFPQ and phosphotau in the granules in vitro as well as in postmortem human brain of rpAD subjects further strengthen the possibility that SFPQ contributes to tau pathology through the formation and/or stabilization of stress granules.

In the present study, immunoblotting analysis of total cell lysates after stress induction also indicated an increase in tau phosphorylation in agreement with immunocytochemical data. Our results suggest that the phosphorylation status and distribution of tau, particularly phospho-tau, are both modified by oxidative stress in HeLa cells. To rule out the possibility of a cell-type-specific increase in tau phosphorylation, we also investigated the phosphorylation status of tau protein in the neuronal cell line SH-SY5Y. A similar increase was observed in tau phosphorylation in SH-SY5Y cells after stress induction. These findings confirm the stress-dependent increase in tau phosphorylation in both neuronal (SH-SY5Y) and non-neuronal (HeLa) cell lines. An increased activity of GSK-3 $\beta$ has been reported in different cell lines leading to hyperphosphorylation of tau after oxidative stress treatment (Feng et al., 2013; Lovell et al., 2004). One of the earliest events occurring in the progression of $A D$ is an elevation in tau phosphorylation (Huang et al., 2016). Our results confirm oxidativestress-induced phosphorylation and redistribution of phospho-tau into cytoplasmic SGs along with SFPQ, providing a possible mechanism for co-aggregation and mislocalization of both proteins (Fig. 38). 


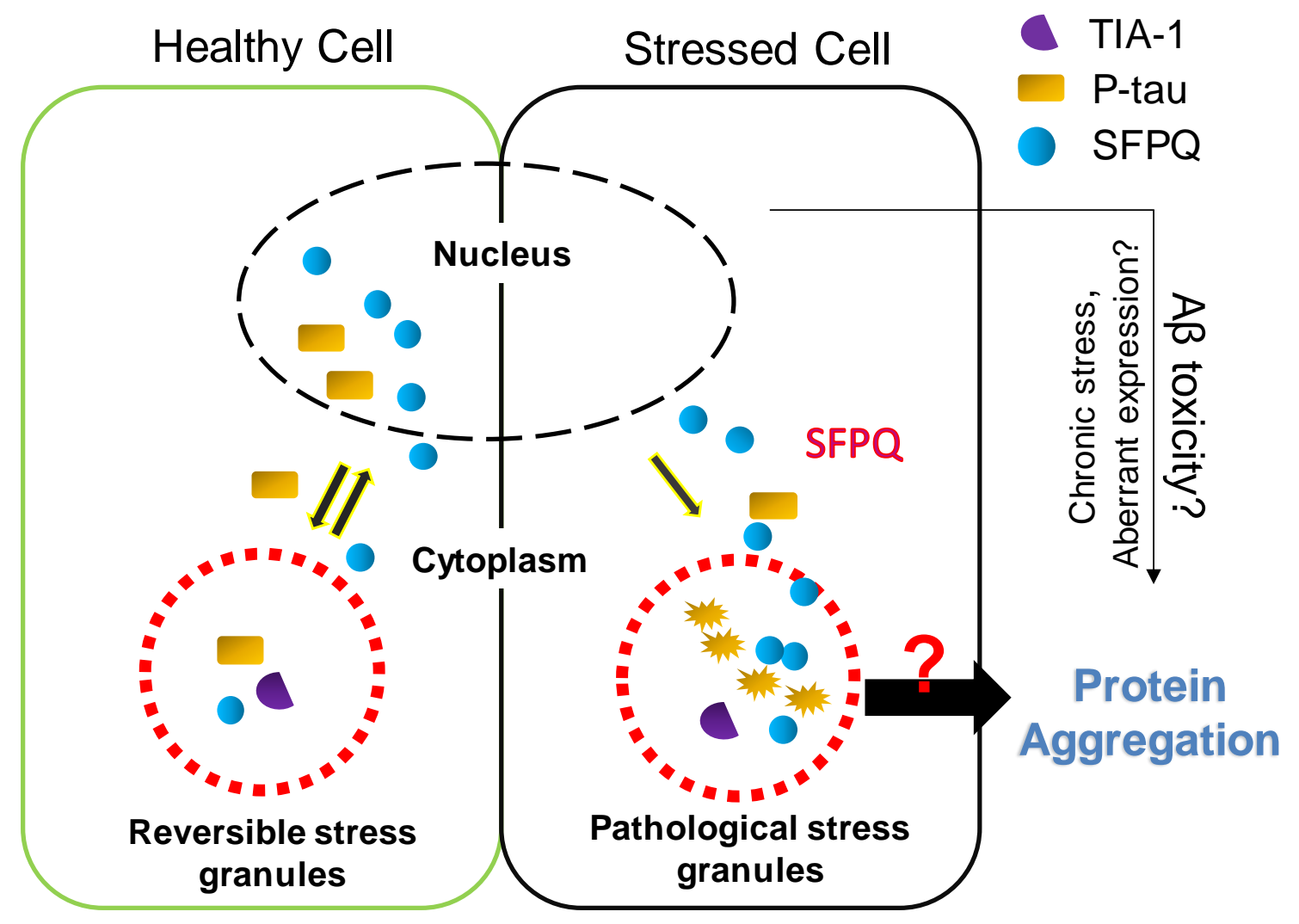

Figure 38: Current working model for SFPQ and tau-pathological features in the rapidly progressive form of Alzheimer's disease. The left box of the picture depicts nucleocytoplasmic translocation of SFPQ and phospho-tau including their assembly into stress granules based on our data from the cellular model of stress. Oxidative-stress-induced redistribution of SFPQ and phospho-tau into the cytoplasm results in the formation of stress granules. These reversible stress granules will be resolved upon removal of the stressors. The right box is depicting pathological features of both proteins observed in the human brain of patients with $\mathrm{rPAD}$ at terminal stage of the disease. A complete nuclear depletion of SFPQ and phospho-tau was observed in the postmortem brain of rPAD cases. Furthermore, SFPQ co-localized with tau tangles, tau oligomers and TIA-1 in the cytoplasm in the human brain and in cultured cells, providing a possible mechanism of SFPQ and nuclear tau dislocation through pathological SGs.

\subsubsection{Biochemical characterization of stress-granule components}

The increase in intensity levels of phospho-tau, TIA-1 and SFPQ during oxidative stress, as observed in this study, raised a concern as to whether this increase was due to increased expression of these proteins or due to consolidation of these proteins into granules. To confirm this, the REAP method (Suzuki et al., 2010) was used, allowing very rapid fractionation (2 $\mathrm{min}$ ) without the involvement of intermediate, time-consumping lysis steps, thus minimizing the chance of technical processing bias in the assessment of protein expression. The REAP method confirmed that there is an increase in tau phosphorylation after oxidative stress. A significant in- 
crease in phospho-tau levels was detected in cytoplasmic and nuclear fractions, with a stronger increase in cytoplasmic fractions. Chronic oxidative stress leads to increased tau phosphorylation in neuronal cultures (Su et al., 2010; Zhu et al. 2005).

Our experiments using biochemical fractionation confirmed that oxidative stress mediates an increase in tau phosphorylation and its redistribution into the cytoplasm. However, the amount of translocated phospho-tau into the cytoplasm was small, as compared to the total tau pool. The levels of TIA-1 increased significantly in nuclear fractions and slightly in whole cell lysate fractions. Furthermore, subcellular fractionation also indicated a slight increase in SFPQ levels in both nuclear and whole cell fractions, although that was not significant. One plausible explanation for this observation could be that the increase in SFPQ levels observed in the total cell lysates was not high enough to be detected in the volume-based normalizations used in the REAP method. In summary, these results confirm increased phosphorylation and redistribution of tau into the cytoplasm in response to oxidative stress. The REAP method also confirmed increase in TIA-1 levels after stress treatment.

\subsubsection{Role of SFPQ towards tau axis}

The Braak-stage-dependent reduction in SFPQ levels in the entorhinal cortex of human brain (Ke et al., 2012) suggests a tau-dependent downregulation of SFPQ. To decipher the role of tau, we expressed recombinant WT or mutant human tau (P301L-tau) in HeLa cells and found a significant increase in total and phosphorylated tau levels after transient transfection. However, there were no significant changes observed in net phosphorylation (phospho-tau/tau) between WT- and P301L tau-expressing cells. Immunoblotting analysis did not show significant differences in the levels of SFPQ 24 hrs post-transfection.

To further extend our knowledge about putative long-term effects of tau expression, we also investigated changes in SFPQ levels 48 hrs post-transfection. Interestingly, immunoblotting analysis revealed a significant reduction in SFPQ levels in WT-tauexpressing cells as compared to controls. These results indicate that expression of tau leads to a reduced SFPQ expression in both WT- and P301L-tau-expressing cells, but a significant downregulation was only found in WT-tau-expressing cells. Downregulation of SFPQ at a transcriptional level has also been reported in pR5 


\section{Discussion}

mice expressing P301L-tau (Ke et al., 2012). Given the reduced SFPQ expression in the human brain frontal cortex of rpAD subjects and in transfeced cells overexpressing tau, our data suggest a tau-dependent modulation of SFPQ expression.

The cell viability assay indicated decreased cell viability in HeLa cells after tauexpression as compared to controls. Increased susceptibility to cell death has been reported after WT-tau expression in other cell lines as well e.g. SY5Y cells (Delobel et al., 2003). Similarly, knockout of whitesnake/sfpq in zebra-fish has been associated with increased apoptosis (Lowery et al., 2007). Furthermore, our immunoblotting analysis indicated that total levels of TIA-1 were not altered under any of these conditions, suggesting that tau has no effect on the total levels of TIA-1. A role of tau in the regulation of the TIA-1 interactome has been reported previously (Vanderweyde et al., 2016). In summary, our data demonstrate that human WT-tau expression leads to SFPQ reduction and higher susceptibility to cell death.

\subsubsection{Dysregulated pathways associated with tau-mediated downregulation of SFPQ}

To explore pathways and mechanisms associated with tau-dependent downregulation of SFPQ, this study employed a quantitative proteomic technology called SWATH-MS. Combination of three functional enrichment strategies (IPA analysis, Fisher's exact test and WebGeStalt-based enrichment analysis) indicated two major themes that were altered: the first was associated with RNA metabolism (RNA damage and repair and cytoplasmic translation via the eiF2 and eiF4 pathways) and the second as associated with DNA damage and repair. Overall, most of the proteins were downregulated (251) after expression of human tau, as compared with the number of up-regulated proteins (63), suggesting a reduction in global translation. Likewise, most of the proteins belonging to the first theme were downregulated. Given the fundamental importance of protein synthesis machinery in the neurons, it is most likely that tau-mediated aberrant synthesis of ribosomal proteins and subunits is harmful to numerous complex neuronal processes (Rangaraju et al., 2017; Slomnicki et al., 2016). Another possible reason for changes in the synthesis of specific sets of proteins, as observed in this study, might be due to aberrant interaction between tau and TIA1, which could sequester specific mRNAs, change the synthesis of particular sets of proteins and, therefore, contribute to tau pathology (Apic- 
co et al., 2018; Vanderweyde et al., 2016). A link between $A D$ and altered or reduced global translation was recognized initially in 1989 (Langstrom et al., 1989). Significant impairment in ribosomal function has been reported in multiple cortical regions in patients with mild cognitive impairment $(\mathrm{MCl})$ and $A D$, due to reduced protein synthesis, reduced ribosomal and transfer RNA levels (Ding et al., 2005; HernandezOrtega et al., 2016). Downregulation of many ribosomal proteins was observed in our data. Recently, impaired synthesis of ribosomal proteins was reported in a tautransgenic mouse model of FTD (Evans et al., 2019) and in cultured cells (Maina et al., 2018). In summary, our findings confirm impaired protein synthesis as a pathomechanism through which pathological tau can disrupt cellular homeostasis.

The second major theme associated with DEPs was DNA damage and repair. Major proteins belonging to this functional category (XRCC5, XRCC6, FEN1, MSH6, POLD1, PCNA, RFC5) exhibited a reduced expression. A malfunction of these proteins associated with "telomere organization and mismatch-repair in eukaryotes" suggests disturbed DNA-metabolic processes. Tau-mediated DNA disorganization has important implications for AD. These findings confirm a toxic role of tau in the nucleus. Previous investigations in $\mathrm{MCl}$ patients have demonstrated a role of DNAdamage in the development of $A D$ and other neurodegenerative diseases (Bucholtz and Demuth, 2013). Based on our results, it is tempting to speculate that tau-induced DNA abnormalities may contribute to AD-related neurological deficits.

There are no reports providing evidence for the involvement of tau in DNA-repair processes (Rossi et al., 2013) or in telomere preservation. A chaperone role for tau protecting genomic DNA against free radicals or heat-induced stress damage has been recently reported (Sultan et al., 2011; Wei et al., 2008), as well as functions in chromatin stabilization (Rossi et al., 2008). Disturbances observed in telomere maintenance and organization could be attributed to a reduction of SFPQ levels, as recently established for SFPQ in the regulation of telomere integrity (Petti et al., 2019).

In summary, the reduced SFPQ levels after tau expression in vitro, in the human brain of rpAD and SCJD subjects, and in the 3xTg-AD animals suggest that this depletion may lead to impaired nuclear DNA functions. As observed in the frontal cortex in this study and in the hippocampus by others (Ke et al., 2012), mislocalization 


\section{Discussion}

of SFPQ suggests a disturbance in multiple roles including DNA damage, transcription, alternative splicing, and transport machinery, eventually contributing to neurodegeneration.

\subsection{Dysregulation of SFPQ, tau, and TIA-1 in 3xTg-AD mice}

To address the physiological significance of reduced expression of SFPQ in the postmortem human brain, the time-dependent expression profile of proteomic signatures was investigated in the model of 3xTg mice both at pre-symptomatic and symptomatic stages of the AD-like disease. The 3xTg-AD mouse model has three mutations (APP Swedish, MAPT P301L, and PSEN1 M146V) in the brain (Oddo et al., 2003) and develops $A \beta$-plaques and tau tangles in the hippocampus from the age of 6 months (Belfiore et al., 2019).

In the current study, we identified a significant reduction in the levels of total tau at late-symptomatic stage of the disease in 3xTg-AD mice as compared to controls. No significant changes were observed for phospho-tau (S199), which was consistent with previous findings in 3xTg-AD mice (Li et al., 2019). Furthermore, we uncovered that SFPQ and TIA-1 were already significantly elevated at the early presymptomatic phase $(3 \mathrm{mpi}$ ) of the disease, which indicates their relevance as early disease-modifying targets in 3xTg-AD mice (Fig. 39). This upregulation in 3xTg-AD mice is consistent with the oxidative-stress-induced increase in SFPQ and TIA-1 levels in HeLa cells (Fig. 39), suggesting that at early stages of the disease pre-tangle pathology might induce these changes (Santacruz et al., 2005).

During progression of the disease, SFPQ levels did not differ from controls at the early symptomatic phase of the disease. This study observed that, in 3xTg-AD mice at $12 \mathrm{mpi}$, there was a drastic reduction in SFPQ levels, comparable to the data from the postmortem human brain tissues from rpAD and SCJD patients. It was demonstrated that overexpression of SFPQ contributes to cell death, since it sensitizes neurons to neurotransmitter-mediated cell death (Xu et al., 2005).

Reduction in TIA-1 (haplosufficiency) has been shown to be protective against neurodegeneration (Apicco et al., 2018). The significant downregulation of TIA-1 observed at the terminal stage in $3 \times \mathrm{Tg}-\mathrm{AD}$ mice and in spAD postmortem brains may 
exhibit a protective or compensatory effect (Fig. 39). The significant increase in TIA1 in rpAD in comparison to spAD suggests higher neurodegeneration in rpAD cases.

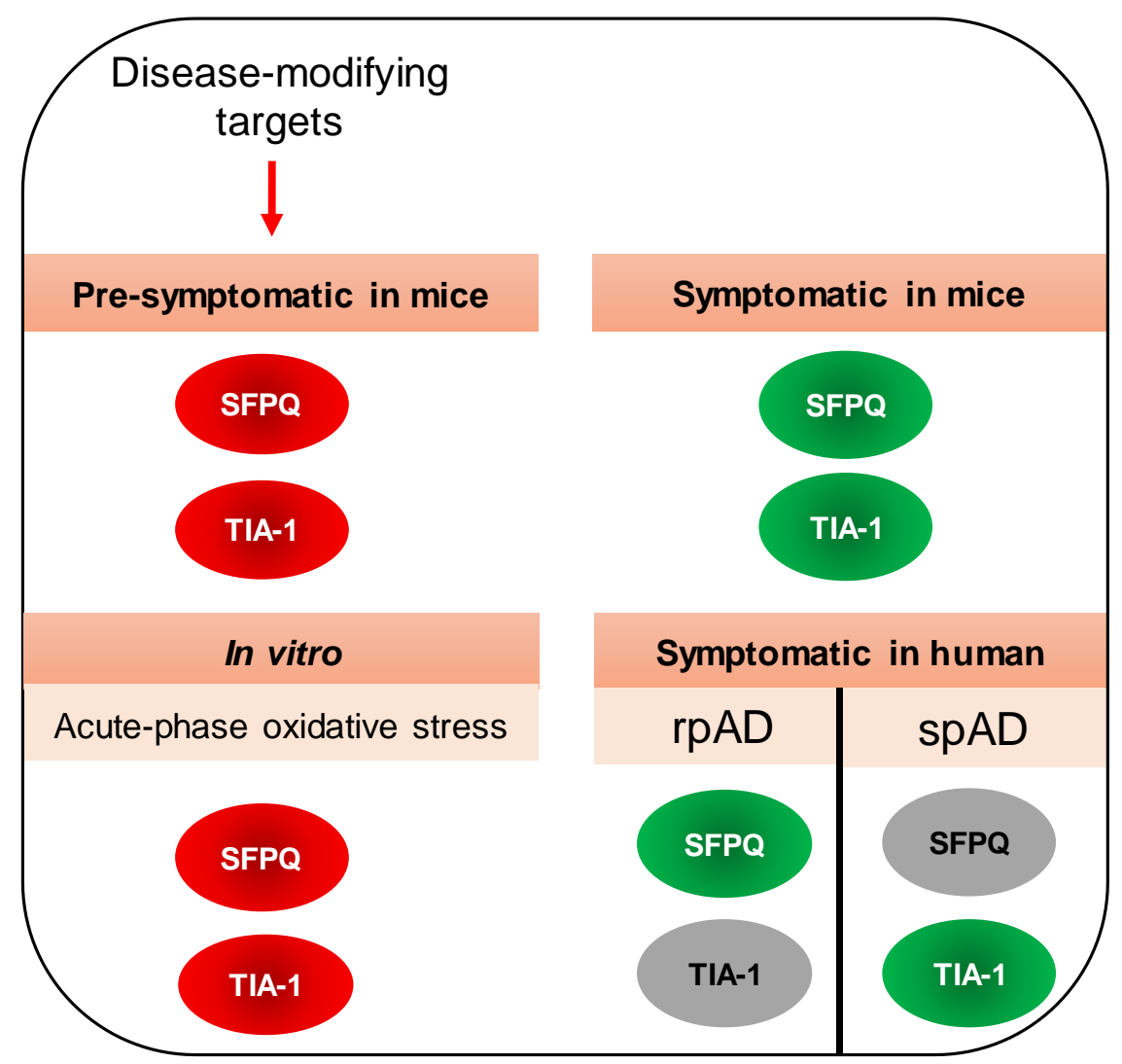

Figure 39: Comparative analysis of the differential expression of SFPQ and TIA-1 in mice and humans. The red boxes are indicating upregulated proteins, whereas the green boxes are representing downregulated proteins, with grey boxes indicating non-significant changes in relation to controls. Expression of SFPQ and TIA-1 was significantly elevated at the early pre-symptomatic phase $(3 \mathrm{mpi})$ of the disease in $3 x \mathrm{Tg}-\mathrm{AD}$ mice, suggesting that these proteins could be of potential significance as early disease-modifying targets. The high expression levels of SFPQ and TIA-1 in mice were also observed in HeLa cells following exposure to oxidative stress. At symptomatic stage of the disease, differential regulation was observed for both SFPQ and TIA-1. The reduced SFPQ levels in the postmortem human brains of rpAD patients was also evident at late symptomatic stage in the 3xTg-AD mice.

In summary, the research findings from the current study confirmd the heterogeneity of $A D$ entities. Proteomic analyses have identified several subtype-specific RBPs, but further studies are required to assess their clinical relevance. 


\subsection{Conclusion}

RNA-binding proteins as key regulators in RNA processing and translational control may have pathophysiological functions in Alzheimer's disease. This study shows that the RNA-binding protein SFPQ (splicing factor proline and glutamine rich) is dysregulated at both the protein and mRNA level in the frontal cortex of patients dignosed with rpAD or SCJD as well as in the brains of $3 \times T g-A D$ mice. Coimmunofluorescence analysis in combination with confocal-laser scanning microscopy demonstrated nuclear depletion of SFPQ along with phospho-tau, particularly in cases of rapidly progressive AD. This nuclear depletion of both proteins was concomitantly associated with their cytoplasmic redistribution. Of note, association between SFPQ and tau in rpAD brain did not exclude the possible role of SFPQ in oligomerization and misfolding of tau protein. In the human brain, immunoreactivity of SFPQ co-localized with cytoplasmic TIA-1, which is a marker of stress granules. A similar translocation of SFPQ and phospho-tau into cytoplasmic TIA-1-positive stress granules was also obtained in cultured HeLa cells treated with sodium arsenite. Furthermore, the expression of human tau in vitro induced a significant reduction in SFPQ levels, suggesting a causal role of tau in downregulation of SFPQ. The findings from the current study indicate that dysregulated SFPQ in combination with pathological tau and aberrant dynamics of SGs represents an important pathway, which may contribute to the rapid progression of $A D$. The re-establishment of the normal localization of SFPQ may be a candidate for future therapeutic research strategies. 


\section{Annexure}

Table 11: Patient details of Alzheimer's disease subtypes and non-demented controls

\begin{tabular}{|c|c|c|c|c|c|c|}
\hline No. & Case & Age & Gender & $\begin{array}{l}\text { Disease } \\
\text { duration }(y)\end{array}$ & $\begin{array}{l}\text { Braak } \\
\text { stages }\end{array}$ & PMI (hr) \\
\hline 1 & rpAD1 & 70 & Male & $<4$ & $\mathrm{VI} / \mathrm{C}$ & $11: 30$ \\
\hline 2 & rpAD2 & 76 & Female & $<4$ & VI & 18 \\
\hline 3 & rpAD3 & 79 & Female & $<4$ & $\mathrm{~V}$ & $05: 30$ \\
\hline 4 & rpAD4 & 83 & Male & $<4$ & $\mathrm{VI} / \mathrm{C}$ & $05: 30$ \\
\hline 5 & rpAD5 & 83 & Male & $<4$ & $\mathrm{~V} / \mathrm{C}$ & 08:20 \\
\hline 6 & rpAD6 & 76 & Male & $<4$ & $\mathrm{VI} / \mathrm{C}$ & $06: 30$ \\
\hline 7 & rpAD7 & 77 & Female & $<4$ & $\mathrm{IV} / \mathrm{A}$ & 12 \\
\hline 8 & rpAD8 & 78 & Male & $<4$ & $\mathrm{VI} / \mathrm{C}$ & 03:30 \\
\hline 9 & spAD1 & 78 & Male & $>4$ & $\mathrm{~V} / \mathrm{C}$ & 09:30 \\
\hline 10 & spAD2 & 72 & Female & $>4$ & $\mathrm{~V} / \mathrm{C}$ & 09:30 \\
\hline 11 & spAD3 & 82 & Female & $>4$ & VI/B & $01: 45$ \\
\hline 12 & spAD4 & 56 & Female & $>4$ & $\mathrm{~V} / \mathrm{C}$ & 07 \\
\hline 13 & spAD5 & 87 & Male & $>4$ & $\mathrm{~V} / \mathrm{C}$ & 07:05 \\
\hline 14 & spAD6 & 75 & Female & $>4$ & $\mathrm{~V} / \mathrm{C}$ & $04: 15$ \\
\hline 15 & spAD7 & 93 & Male & $>4$ & $\mathrm{~V} / \mathrm{C}$ & 03 \\
\hline 16 & spAD8 & 67 & Female & $>4$ & III/C & $06: 10$ \\
\hline 17 & spAD9 & 90 & Female & $>4$ & $\mathrm{IV} / \mathrm{A}$ & $09: 55$ \\
\hline 18 & spAD10 & 83 & Male & $>4$ & III/0 & $07: 25$ \\
\hline 19 & Cont.1 & 69 & Male & - & II/A & $03: 45$ \\
\hline 20 & Cont.2 & 68 & Male & - & $\mathrm{I} / 0$ & $10: 55$ \\
\hline 21 & Cont.3 & 64 & Male & - & $\mathrm{I} / 0$ & $08: 35$ \\
\hline 22 & Cont.4 & 67 & Male & - & $\mathrm{I} / 0$ & $14: 40$ \\
\hline 23 & Cont.5 & 74 & Male & - & II/A & $05: 30$ \\
\hline 24 & Cont. 6 & 86 & Male & - & II/A & 05:30 \\
\hline 25 & Cont.7 & 73 & Female & - & $\mathrm{I} / 0$ & $15: 45$ \\
\hline 26 & Cont. 8 & 61 & Male & - & 1 & $04: 30$ \\
\hline 27 & Cont.9 & 77 & Male & - & $\mathrm{I} / \mathrm{A}$ & $06: 55$ \\
\hline
\end{tabular}

PMI (hr): Postmortem interval in hours, rpAD: rapidly progressive Alzheimer's disease, spAD: sporadic Alzheimer' disease.

Table 12: Details of sporadic Creutzfeldt Jakob disease subtype cases

\begin{tabular}{lllllll}
\hline No. & Case & Age & Gender & $\begin{array}{l}\text { Disease } \\
\text { duration }(\mathbf{y})\end{array}$ & Genotype & PMI (hr) \\
\hline 1 & sCJD (MM1)1 & 65 & Male & $<1$ & MM/MV1 & $09: 45$ \\
2 & sCJD (MM1)2 & 74 & Female & $<1$ & MM/MV1 & $07: 50$ \\
3 & sCJD (MM1)3 & 61 & Male & $<1$ & MM/MV1 & 07 \\
4 & sCJD (MM1)4 & 66 & Female & $<1$ & MM/MV1 & $05: 05$ \\
5 & sCJD (MM1)5 & 74 & Female & $<1$ & MM/MV1 & 11 \\
6 & sCJD (MM1)6 & 74 & Male & $<1$ & MM/MV1 & $04: 50$ \\
7 & sCJD (VV2)1 & 66 & Male & $<1$ & VV2 & $15: 30$ \\
8 & SCJD (VV2)2 & 70 & Female & $<1$ & VV2 & 11 \\
9 & sCJD (VV2)3 & 72 & Female & $<1$ & VV2 & 06 \\
10 & sCJD (VV2)4 & 66 & Female & $<1$ & VV2 & 04 \\
\hline
\end{tabular}


Table 13: Details of cases used for immunohistochemistry analysis

\begin{tabular}{llllll}
\hline No. & Case & Age & Gender & $\begin{array}{l}\text { Disease dura- } \\
\text { tion }(\mathbf{y})\end{array}$ & ABC score \\
\hline 1 & rpAD1 & 59 & Female & $<4$ & A3, B3, C3 \\
2 & rpAD2 & 88 & Female & $<4$ & A3, B2, C3 \\
3 & rpAD3 & 76 & Female & $<4$ & A3, B3, C2 \\
4 & rpAD4 & 71 & Female & $<4$ & A2, B3, C3 \\
5 & rpAD5 & 84 & Male & $<4$ & A2, B3, C3 \\
6 & spAD1 & 87 & Male & $>4$ & A1, B3, C3 \\
7 & spAD2 & 77 & Female & $>4$ & A1, B3, C2 \\
8 & spAD3 & 69 & Female & $>4$ & A1, B2, C3 \\
9 & spAD4 & 62 & Female & $>4$ & A1, B1, C2 \\
10 & spAD5 & 85 & Male & $>4$ & A2, B3, C3 \\
11 & Cont.1 & 74 & Female & - & - \\
12 & Cont.2 & 87 & Female & - & - \\
13 & Cont.3 & 84 & Female & - & - \\
14 & Cont.4 & 82 & Male & - & - \\
15 & Cont.5 & 75 & Male & - & - \\
\hline
\end{tabular}

Table 14: List of primer pairs used in the study

\begin{tabular}{cll}
\hline Gene name & Direction & \multicolumn{1}{c}{ Sequence } \\
\hline & Forward & TGGGTGTGAACCATGAGAAGTA \\
\cline { 2 - 3 } GAPDH & Reverse & GAGTCCTTCCACGATACCAAAG \\
\cline { 2 - 3 } SFPQ & Forward & TGGGAAGTGACATGCGTACT \\
& Reverse & TGTTTGGGCCTTCGTACTCT \\
& Forward & AGTTTCCTGGCCTGCATTTC \\
\hline TIA-1 & Reverse & ACACTCGAGCTGTCTTTCCT \\
\cline { 2 - 3 } $\boldsymbol{V C P}$ & Forward & AAACGTATCCATGTGCTGCC \\
\hline & Reverse & ACTTTGACTCCACAGCACG \\
\hline
\end{tabular}

\subsection{RNA-binding protein candidates from mass spectrometry analysis.}

Table 15: List of unique and common RNA-binding protein candidates identified in sporadic $A D$, rapidly progressive $A D$, and sporadic Creutzfeldt-Jakob disease

\begin{tabular}{|c|c|c|c|c|}
\hline $\begin{array}{l}\text { Disease } \\
\text { Group }\end{array}$ & IDs & $\begin{array}{l}\text { Uniprot } \\
\text { Acc. No. }\end{array}$ & Protein names & $\begin{array}{c}\text { Involvement in } \\
\text { disease }\end{array}$ \\
\hline \multirow{8}{*}{ spAD } & AEDO & Q96SZ5 & 2-aminoethanethiol dioxygenase & \\
\hline & AL1L1 & O75891 & $\begin{array}{l}\text { Cytosolic } 10 \text {-formyltetrahydrofolate } \\
\text { dehydrogenase }\end{array}$ & \\
\hline & AL4A1 & P30038 & $\begin{array}{l}\text { Delta-1-pyrroline-5-carboxylate } \\
\text { dehydrogenase, mitochondrial }\end{array}$ & Hyperprolinemia 2 \\
\hline & AP2M1 & Q96CW1 & AP-2 complex subunit mu & \\
\hline & ARP2 & P61160 & Actin-related protein 2 & \\
\hline & ARRB1 & P49407 & Beta-arrestin-1 & \\
\hline & ATPD & P30049 & $\begin{array}{l}\text { ATP synthase subunit delta, } \\
\text { mitochondrial }\end{array}$ & $\begin{array}{l}\text { Mitochondrial com- } \\
\text { plex V deficiency, } \\
\text { nuclear tvpe } 5\end{array}$ \\
\hline & CAD13 & P55290 & Cadherin-13 & \\
\hline
\end{tabular}




\begin{tabular}{|c|c|c|c|}
\hline CADM4 & Q8NFZ8 & Cell adhesion molecule 4 & \\
\hline CANB1 & P63098 & Calcineurin subunit B type 1 & \\
\hline CAND1 & Q86VP6 & $\begin{array}{l}\text { Cullin-associated NEDD8-dissociated } \\
\text { protein } 1\end{array}$ & \\
\hline CAPZB & P47756 & F-actin-capping protein subunit beta & \\
\hline CASA1 & P47710 & $\begin{array}{l}\text { Alpha-S1-casein [Cleaved into: Casoxin- } \\
\text { D] }\end{array}$ & \\
\hline CAZA1 & P52907 & F-actin-capping protein subunit alpha-1 & \\
\hline CC50A & Q9NV96 & Cell cycle control protein $50 \mathrm{~A}$ & \\
\hline CD47 & Q08722 & Leukocyte surface antigen CD47 & \\
\hline CLCA & P09496 & Clathrin light chain A & \\
\hline CLH2 & P53675 & Clathrin heavy chain 2 & \\
\hline CPLX2 & Q6PUV4 & Complexin-2 & \\
\hline CSN2 & P61201 & COP9 signalosome complex subunit 2 & \\
\hline CYFP2 & Q96F07 & Cytoplasmic FMR1-interacting protein 2 & $\begin{array}{l}\text { Epileptic } \\
\text { encephalopathy, } \\
\text { early infantile, } 65\end{array}$ \\
\hline DBNL & Q9UJU6 & Drebrin-like protein & \\
\hline DC1L2 & O43237 & $\begin{array}{l}\text { Cytoplasmic dynein } 1 \text { light intermediate } \\
\text { chain } 2\end{array}$ & \\
\hline DCXR & Q7Z4W1 & L-xylulose reductase & Pentosuria \\
\hline ECHA & P40939 & $\begin{array}{l}\text { Trifunctional enzyme subunit alpha, mi- } \\
\text { tochondrial }\end{array}$ & $\begin{array}{l}\text { Mitochondrial } \\
\text { trifunctional protein } \\
\text { deficiency }\end{array}$ \\
\hline ENOB & P13929 & Beta-enolase & $\begin{array}{l}\text { Glycogen storage } \\
\text { disease } 13\end{array}$ \\
\hline FLNA & P21333 & Filamin-A & $\begin{array}{l}\text { Periventricular } \\
\text { nodular heterotopia } \\
1\end{array}$ \\
\hline FLOT1 & O75955 & Flotillin-1 & \\
\hline FUBP2 & Q92945 & Far upstream element-binding protein 2 & \\
\hline GANAB & Q14697 & Neutral alpha-glucosidase AB & $\begin{array}{l}\text { Polycystic kidney } \\
\text { disease } 3 \text { with or } \\
\text { without polycystic } \\
\text { liver disease }\end{array}$ \\
\hline GBB4 & Q9HAV0 & $\begin{array}{l}\text { Guanine nucleotide-binding protein sub- } \\
\text { unit beta-4 }\end{array}$ & $\begin{array}{l}\text { Charcot-Marie- } \\
\text { Tooth disease, dom- } \\
\text { inant, intermediate } \\
\text { type, } \mathrm{F}\end{array}$ \\
\hline GBG3 & P63215 & Guanine nucleotide-binding protein G & \\
\hline GDE & P35573 & Glycogen debranching enzyme & $\begin{array}{l}\text { Glycogen storage } \\
\text { disease } 3\end{array}$ \\
\hline GLU2B & P14314 & Glucosidase 2 subunit beta & $\begin{array}{l}\text { Polycystic liver dis- } \\
\text { ease } 1 \text { with or with- } \\
\text { out kidney cysts }\end{array}$ \\
\hline GNAQ & P50148 & Guanine nucleotide-binding protein $\mathrm{G}$ & $\begin{array}{l}\text { Capillary } \\
\text { malformations, } \\
\text { congenital }\end{array}$ \\
\hline GPD1L & Q8N335 & $\begin{array}{l}\text { Glycerol-3-phosphate dehydrogenase 1- } \\
\text { like protein }\end{array}$ & $\begin{array}{l}\text { Brugada syndrome } \\
2\end{array}$ \\
\hline GPDM & P43304 & $\begin{array}{l}\text { Glycerol-3-phosphate dehydrogenase, } \\
\text { mitochondrial }\end{array}$ & \\
\hline HINT1 & P49773 & $\begin{array}{l}\text { Histidine triad nucleotide-binding protein } \\
1\end{array}$ & $\begin{array}{l}\text { Neuromyotonia and } \\
\text { axonal neuropathy, } \\
\text { autosomal recessive }\end{array}$ \\
\hline
\end{tabular}




\begin{tabular}{|c|c|c|c|}
\hline HSP76 & P17066 & Heat shock 70 kDa protein 6 & \\
\hline ICAM5 & Q9UMF0 & Intercellular adhesion molecule 5 & \\
\hline IDH3B & O43837 & $\begin{array}{l}\text { Isocitrate dehydrogenase [NAD] subunit } \\
\text { beta, mitochondrial }\end{array}$ & $\begin{array}{l}\text { Retinitis pigmentosa } \\
46\end{array}$ \\
\hline IF4A1 & P60842 & Eukaryotic initiation factor $4 \mathrm{~A}-\mathrm{I}$ & \\
\hline IF4B & P23588 & Eukaryotic translation initiation factor 4B & \\
\hline IPYR & Q15181 & Inorganic pyrophosphatase & \\
\hline KCC2A & Q9UQM7 & $\begin{array}{l}\text { Calcium/calmodulin-dependent protein } \\
\text { kinase type II subunit alpha }\end{array}$ & $\begin{array}{l}\text { Mental retardation, } \\
\text { autosomal dominant } \\
53\end{array}$ \\
\hline KCRM & P06732 & Creatine kinase M-type & \\
\hline KT3K & Q9HA64 & Ketosamine-3-kinase & \\
\hline L1CAM & P32004 & Neural cell adhesion molecule L1 & $\begin{array}{l}\text { Hydrocephalus due } \\
\text { to stenosis of the } \\
\text { aqueduct of Sylvius }\end{array}$ \\
\hline LASP1 & Q14847 & LIM and SH3 domain protein 1 & \\
\hline LIG01 & Q96FE5 & $\begin{array}{l}\text { Leucine-rich repeat and immunoglobulin- } \\
\text { like domain-containing nogo receptor- } \\
\text { interacting protein } 1\end{array}$ & $\begin{array}{l}\text { Mental retardation, } \\
\text { autosomal recessive } \\
64\end{array}$ \\
\hline MAOM & P23368 & $\begin{array}{l}\text { NAD-dependent malic enzyme, } \\
\text { mitochondrial }\end{array}$ & \\
\hline MAP4 & P27816 & Microtubule-associated protein 4 & \\
\hline MK03 & P27361 & Mitogen-activated protein kinase 3 & \\
\hline MT1F & P04733 & Metallothionein-1F & \\
\hline NCHL1 & 000533 & $\begin{array}{l}\text { Neural cell adhesion molecule L1-like } \\
\text { protein }\end{array}$ & \\
\hline NCKP1 & Q9Y2A7 & Nck-associated protein 1 & \\
\hline NCKX2 & Q9U140 & Sodium/potassium/calcium exchanger 2 & \\
\hline NDRG1 & Q92597 & Protein NDRG1 & $\begin{array}{l}\text { Charcot-Marie- } \\
\text { Tooth disease 4D }\end{array}$ \\
\hline NEDD8 & Q15843 & NEDD8 & \\
\hline NEGR1 & Q7Z3B1 & Neuronal growth regulator 1 & \\
\hline NP1L4 & Q99733 & Nucleosome assembly protein 1-like 4 & \\
\hline NTRI & Q9P121 & Neurotrimin & \\
\hline ODPA & P08559 & $\begin{array}{l}\text { Pyruvate dehydrogenase E1 component } \\
\text { subunit alpha, somatic form, } \\
\text { mitochondrial }\end{array}$ & $\begin{array}{l}\text { Pyruvate } \\
\text { dehydrogenase E1- } \\
\text { alpha deficiency }\end{array}$ \\
\hline OLA1 & Q9NTK5 & Obg-like ATPase 1 & \\
\hline OPA1 & O60313 & $\begin{array}{l}\text { Dynamin-like } 120 \text { kDa protein, } \\
\text { mitochondrial }\end{array}$ & Optic atrophy 1 \\
\hline OPCM & Q14982 & $\begin{array}{l}\text { Opioid-binding protein/cell adhesion } \\
\text { molecule }\end{array}$ & Ovarian cancer \\
\hline PAK1 & Q13153 & Serine/threonine-protein kinase PAK 1 & $\begin{array}{l}\text { Intellectual devel- } \\
\text { opmental disorder } \\
\text { with macrocephaly, } \\
\text { seizures, and } \\
\text { speech delay }\end{array}$ \\
\hline PAK3 & О75914 & Serine/threonine-protein kinase PAK 3 & $\begin{array}{l}\text { Mental retardation, } \\
\text { X-linked } 30\end{array}$ \\
\hline PCSK1 & Q9UHG2 & ProSAAS & \\
\hline PCY2 & Q99447 & $\begin{array}{l}\text { Ethanolamine-phosphate } \\
\text { cytidylyltransferase }\end{array}$ & \\
\hline PFKAL & P17858 & $\begin{array}{l}\text { ATP-dependent 6-phosphofructokinase, } \\
\text { liver type }\end{array}$ & \\
\hline PFKAP & Q01813 & ATP-dependent 6-phosphofructokinase, & \\
\hline
\end{tabular}




\begin{tabular}{|c|c|c|c|c|}
\hline & & & platelet type & \\
\hline & PHIPL & Q96FC7 & $\begin{array}{l}\text { Phytanoyl-CoA hydroxylase-interacting } \\
\text { protein-like }\end{array}$ & \\
\hline & PLIN3 & O60664 & Perilipin-3 & \\
\hline & PPT1 & P50897 & Palmitoyl-protein thioesterase 1 & $\begin{array}{l}\text { Ceroid } \\
\text { lipofuscinosis, } \\
\text { neuronal, } 1\end{array}$ \\
\hline & PTN11 & Q06124 & $\begin{array}{l}\text { Tyrosine-protein phosphatase non- } \\
\text { receptor type } 11\end{array}$ & $\begin{array}{l}\text { LEOPARD } \\
\text { syndrome } 1\end{array}$ \\
\hline & QCR2 & P22695 & $\begin{array}{l}\text { Cytochrome b-c1 complex subunit } 2 \text {, } \\
\text { mitochondrial }\end{array}$ & $\begin{array}{l}\text { Mitochondrial com- } \\
\text { plex III deficiency, } \\
\text { nuclear } 5\end{array}$ \\
\hline & RAB8B & Q92930 & Ras-related protein Rab-8B & \\
\hline & RALA & P11233 & Ras-related protein Ral-A & \\
\hline & RD23B & P54727 & $\begin{array}{l}\text { UV excision repair protein RAD23 homo- } \\
\text { log B }\end{array}$ & \\
\hline & REEP5 & Q00765 & Receptor expression-enhancing protein 5 & \\
\hline & RHOC & P08134 & Rho-related GTP-binding protein RhoC & \\
\hline & SEMG1 & P04279 & Semenogelin-1 & \\
\hline & Septin-3 & Q9UH03 & Neuronal-specific septin-3 & \\
\hline & Septin-6 & Q14141 & Septin-6 & \\
\hline & Septin-9 & Q9UHD8 & Septin-9 & \\
\hline & SH3G1 & Q99961 & Endophilin-A2 & \\
\hline & SHLB2 & Q9NR46 & Endophilin-B2 & \\
\hline & SNAG & Q99747 & Gamma-soluble NSF attachment protein & \\
\hline & SPTN2 & O15020 & Spectrin beta chain, non-erythrocytic 2 & $\begin{array}{l}\text { Spinocerebellar } \\
\text { ataxia } 5\end{array}$ \\
\hline & SRC8 & Q14247 & Src substrate cortactin & \\
\hline & SYNPO & Q8N3V7 & Synaptopodin & \\
\hline & TCAL5 & Q5H9L2 & $\begin{array}{l}\text { Transcription elongation factor A protein- } \\
\text { like } 5\end{array}$ & \\
\hline & TCPB & P78371 & T-complex protein 1 subunit beta & \\
\hline & TCPQ & P50990 & T-complex protein 1 subunit theta & \\
\hline & TCTP & P13693 & Translationally-controlled tumor protein & \\
\hline rpAD & 4F2 & P08195 & 4F2 cell-surface antigen heavy chain & \\
\hline & ACTA & P62736 & Actin, aortic smooth muscle & \\
\hline & ACY2 & P45381 & Aspartoacylase & Canavan disease \\
\hline & ADDA & P35611 & Alpha-adducin & \\
\hline & ADT2 & P05141 & ADP/ATP translocase 2 & \\
\hline & AL7A1 & P49419 & $\begin{array}{l}\text { Alpha-aminoadipic semialdehyde } \\
\text { dehydrogenase }\end{array}$ & $\begin{array}{l}\text { Pyridoxine- } \\
\text { dependent epilepsy }\end{array}$ \\
\hline & AMER2 & Q8N7J2 & APC membrane recruitment protein 2 & \\
\hline & AMPL & P28838 & Cytosol aminopeptidase & \\
\hline & ANK2 & Q01484 & Ankyrin-2 & $\begin{array}{l}\text { Long QT syndrome } \\
4\end{array}$ \\
\hline & AOFA & P21397 & Amine oxidase [flavin-containing] A & Brunner syndrome \\
\hline & AOFB & P27338 & Amine oxidase [flavin-containing] B & \\
\hline & AP180 & 060641 & Clathrin coat assembly protein AP180 & \\
\hline & ASAH1 & Q13510 & Acid ceramidase & $\begin{array}{l}\text { Farber } \\
\text { lipogranulomatosis }\end{array}$ \\
\hline & AT2A2 & P16615 & $\begin{array}{l}\text { Sarcoplasmic/endoplasmic reticulum } \\
\text { calcium ATPase } 2\end{array}$ & $\begin{array}{l}\text { Acrokeratosis } \\
\text { verruciformis }\end{array}$ \\
\hline & ATP5H & O75947 & ATP synthase subunit $d$, mitochondrial & \\
\hline
\end{tabular}




\begin{tabular}{|c|c|c|c|}
\hline ATP5J & P18859 & $\begin{array}{l}\text { ATP synthase-coupling factor } 6 \text {, mito- } \\
\text { chondrial }\end{array}$ & \\
\hline ATP5L & O75964 & ATP synthase subunit $g$, mitochondrial & \\
\hline CALX & P27824 & Calnexin & \\
\hline CD44 & P16070 & CD44 antigen & \\
\hline CDS2 & O95674 & Phosphatidate cytidylyltransferase 2 & \\
\hline CLD11 & O75508 & Claudin-11 & \\
\hline CMC1 & O75746 & $\begin{array}{l}\text { Calcium-binding mitochondrial carrier } \\
\text { protein Aralar1 }\end{array}$ & $\begin{array}{l}\text { Epileptic } \\
\text { encephalopathy, } \\
\text { early infantile, } 39\end{array}$ \\
\hline CO4A & POCOL4 & Complement C4-A & $\begin{array}{l}\text { Complement } \\
\text { component } 4 \mathrm{~A} \\
\text { deficiency }\end{array}$ \\
\hline CO4B & P0C0L5 & Complement C4-B & $\begin{array}{l}\text { Systemic lupus } \\
\text { erythematosus }\end{array}$ \\
\hline CoX41 & P13073 & $\begin{array}{l}\text { Cytochrome } \mathrm{c} \text { oxidase subunit } 4 \text { isoform } \\
1 \text {, mitochondrial }\end{array}$ & \\
\hline CPNS1 & P04632 & Calpain small subunit 1 & \\
\hline CUTA & O60888 & Protein CutA & \\
\hline DC112 & Q13409 & $\begin{array}{l}\text { Cytoplasmic dynein } 1 \text { intermediate chain } \\
2\end{array}$ & \\
\hline DDTL & A6NHG4 & $\begin{array}{l}\text { D-dopachrome decarboxylase-like pro- } \\
\text { tein }\end{array}$ & \\
\hline EF2 & P13639 & Elongation factor 2 & $\begin{array}{l}\text { Spinocerebellar } \\
\text { ataxia } 26\end{array}$ \\
\hline ERMIN & Q8TAM6 & Ermin & \\
\hline FIS1 & Q9Y3D6 & Mitochondrial fission 1 protein & \\
\hline GBG2 & P59768 & Guanine nucleotide-binding protein $\mathrm{G}$ & \\
\hline GHC1 & Q9H936 & Mitochondrial glutamate carrier 1 & $\begin{array}{l}\text { Epileptic } \\
\text { encephalopathy, } \\
\text { early infantile, } 3\end{array}$ \\
\hline GPM6B & Q13491 & Neuronal membrane glycoprotein M6-b & \\
\hline HEBP1 & Q9NRV9 & Heme-binding protein 1 & \\
\hline HNRPD & Q14103 & $\begin{array}{l}\text { Heterogeneous nuclear } \\
\text { ribonucleoprotein D0 }\end{array}$ & \\
\hline HYEP & P07099 & Epoxide hydrolase 1 & \\
\hline IMB1 & Q14974 & Importin subunit beta-1 & \\
\hline LAMP1 & P11279 & $\begin{array}{l}\text { Lysosome-associated membrane } \\
\text { glycoprotein } 1\end{array}$ & \\
\hline LANC2 & Q9NS86 & LanC-like protein 2 & \\
\hline MAG & P20916 & Myelin-associated glycoprotein & $\begin{array}{l}\text { Spastic paraplegia } \\
75 \text {, autosomal } \\
\text { recessive }\end{array}$ \\
\hline MRP & P49006 & MARCKS-related protein & \\
\hline MTAP2 & P11137 & Microtubule-associated protein 2 & \\
\hline MTPN & P58546 & Myotrophin & \\
\hline NDKA & P15531 & Nucleoside diphosphate kinase A & \\
\hline NDUA4 & O00483 & Cytochrome c oxidase subunit NDUFA4 & Leigh syndrome \\
\hline ODO2 & P36957 & $\begin{array}{l}\text { Dihydrolipoyllysine-residue succinyltrans- } \\
\text { ferase component of 2-oxoglutarate de- } \\
\text { hydrogenase complex, mitochondrial }\end{array}$ & $\begin{array}{l}\text { Platelet-activating } \\
\text { factor acetylhydro- } \\
\text { lase IB subunit beta }\end{array}$ \\
\hline PA1B2 & P68402 & & \\
\hline PCBP2 & Q15366 & Poly(rC)-binding protein 2 & \\
\hline PGAM2 & P15259 & Phosphoglycerate mutase 2 & $\begin{array}{l}\text { Glycogen storage } \\
\text { disease } 10\end{array}$ \\
\hline
\end{tabular}




\begin{tabular}{|c|c|c|c|c|}
\hline & PHB & P35232 & Prohibitin & \\
\hline & PHB2 & Q99623 & Prohibitin-2 & \\
\hline & PI42A & P48426 & $\begin{array}{l}\text { Phosphatidylinositol 5-phosphate 4- } \\
\text { kinase type-2 alpha }\end{array}$ & \\
\hline & PRIO & P04156 & Major prion protein & $\begin{array}{l}\text { prion diseases, like: } \\
\text { Creutzfeldt-Jakob } \\
\text { disease }\end{array}$ \\
\hline & QCR1 & P31930 & $\begin{array}{l}\text { Cytochrome b-c1 complex subunit } 1 \text {, } \\
\text { mitochondrial }\end{array}$ & \\
\hline & QCR6 & P07919 & $\begin{array}{l}\text { Cytochrome b-c1 complex subunit } 6 \text {, } \\
\text { mitochondrial }\end{array}$ & \\
\hline & RAP1A & P62834 & Ras-related protein Rap-1A & \\
\hline & SCG1 & P05060 & Secretogranin-1 & \\
\hline & SCG2 & P13521 & Secretogranin-2 & \\
\hline & SDHA & P31040 & $\begin{array}{l}\text { Succinate dehydrogenase [ubiquinone] } \\
\text { flavoprotein subunit, mitochondrial }\end{array}$ & $\begin{array}{l}\text { Mitochondrial } \\
\text { complex II } \\
\text { deficiency }\end{array}$ \\
\hline & Septin-4 & O43236 & Septin-4 & \\
\hline & SERA & O43175 & D-3-phosphoglycerate dehydrogenase & $\begin{array}{l}\text { Phosphoglycerate } \\
\text { dehydrogenase } \\
\text { deficiency }\end{array}$ \\
\hline & SIRB1 & O00241 & Signal-regulatory protein beta-1 & \\
\hline & SNG3 & O43761 & Synaptogyrin-3 & \\
\hline & SV2A & Q7L0J3 & Synaptic vesicle glycoprotein $2 \mathrm{~A}$ & \\
\hline & SV2B & Q7L1I2 & Synaptic vesicle glycoprotein 2B & \\
\hline & SYNJ1 & O43426 & Synaptojanin-1 & $\begin{array}{l}\text { Parkinson disease } \\
20 \text {, early-onset }\end{array}$ \\
\hline & TBA1B & P68363 & Tubulin alpha-1B chain & \\
\hline & TCPE & P48643 & T-complex protein 1 subunit epsilon & $\begin{array}{l}\text { Neuropathy, heredi- } \\
\text { tary sensory, with } \\
\text { spastic paraplegia, } \\
\text { autosomal recessive }\end{array}$ \\
\hline & TENA & P24821 & Tenascin & $\begin{array}{l}\text { Deafness, } \\
\text { autosomal } \\
\text { dominant, } 56\end{array}$ \\
\hline & VA0D1 & P61421 & V-type proton ATPase subunit d 1 & \\
\hline & VAT1 & Q99536 & $\begin{array}{l}\text { Synaptic vesicle membrane protein VAT- } \\
1 \text { homolog } \\
\text { Voltage-dependent anion-selective } \\
\text { channel protein } 1\end{array}$ & $\begin{array}{l}\text { Voltage-dependent } \\
\text { anion-selective } \\
\text { channel protein } 2 \\
\text { Voltage-dependent } \\
\text { anion-selective } \\
\text { channel protein } 3\end{array}$ \\
\hline & VDAC1 & P21796 & & \\
\hline & VDAC2 & P45880 & & \\
\hline & VDAC3 & Q9Y277 & & \\
\hline sCJD & A2MG & P01023 & Alpha-2-macroglobulin & \\
\hline & AACT & P01011 & Alpha-1-antichymotrypsin & \\
\hline & AC013 & Q9NPJ3 & Acyl-coenzyme A thioesterase 13 & \\
\hline & ADHX & P11766 & Alcohol dehydrogenase class-3 & \\
\hline & AL1A1 & P00352 & Retinal dehydrogenase 1 & \\
\hline & ALDR & P15121 & Aldo-keto reductase family 1 member B1 & \\
\hline & APEX1 & P27695 & DNA-(apurinic or apyrimidinic site) lyase & \\
\hline & APOA1 & $\mathrm{P} 02647$ & Apolipoprotein A-I & $\begin{array}{l}\text { High density } \\
\text { lipoprotein } \\
\text { deficiency } 2\end{array}$ \\
\hline
\end{tabular}




\begin{tabular}{|c|c|c|c|}
\hline ARP3 & P61158 & Actin-related protein 3 & \\
\hline ASGL1 & Q7L266 & Isoaspartyl peptidase/L-asparaginase & \\
\hline BIEA & P53004 & Biliverdin reductase $A$ & Hyperbiliverdinemia \\
\hline CAB39 & Q9Y376 & Calcium-binding protein 39 & \\
\hline CATA & P04040 & Catalase & Acatalasemia \\
\hline CD59 & P13987 & CD59 glycoprotein & $\begin{array}{l}\text { Hemolytic anemia, } \\
\text { CD59-mediated, } \\
\text { with or without poly- } \\
\text { neuropathy }\end{array}$ \\
\hline CLIC4 & Q9Y696 & Chloride intracellular channel protein 4 & \\
\hline CMBL & Q96DG6 & Carboxymethylenebutenolidase homolog & \\
\hline CPPED & Q9BRF8 & $\begin{array}{l}\text { Serine/threonine-protein phosphatase } \\
\text { CPPED1 }\end{array}$ & \\
\hline DOPD & P30046 & D-dopachrome decarboxylase & \\
\hline DYL2 & Q96FJ2 & Dynein light chain 2, cytoplasmic & \\
\hline EFHD2 & Q96C19 & EF-hand domain-containing protein D2 & \\
\hline FABP7 & O15540 & Fatty acid-binding protein, brain & \\
\hline FAHD1 & Q6P587 & Acylpyruvase FAHD1, mitochondrial & \\
\hline FBX2 & Q9UK22 & F-box only protein 2 & \\
\hline FIBB & P02675 & Fibrinogen beta chain & $\begin{array}{l}\text { Congenital } \\
\text { afibrinogenemia }\end{array}$ \\
\hline FIBG & P02679 & Fibrinogen gamma chain & $\begin{array}{l}\text { Congenital } \\
\text { afibrinogenemia }\end{array}$ \\
\hline FKBP4 & Q02790 & $\begin{array}{l}\text { Peptidyl-prolyl cis-trans isomerase } \\
\text { FKBP4 }\end{array}$ & \\
\hline G6PD & P11413 & Glucose-6-phosphate 1-dehydrogenase & $\begin{array}{l}\text { Anemia, non- } \\
\text { spherocytic hemolyt- } \\
\text { ic, due to G6PD } \\
\text { deficiency }\end{array}$ \\
\hline GBRL2 & P60520 & $\begin{array}{l}\text { Gamma-aminobutyric acid receptor- } \\
\text { associated protein-like } 2\end{array}$ & \\
\hline GGCT & O75223 & Gamma-glutamylcyclotransferase & \\
\hline GNAI2 & P04899 & Guanine nucleotide-binding protein G & \\
\hline GNPI1 & P46926 & Glucosamine-6-phosphate isomerase 1 & \\
\hline GSHB & P48637 & Glutathione synthetase & $\begin{array}{l}\text { Glutathione } \\
\text { synthetase } \\
\text { deficiency }\end{array}$ \\
\hline HMGB1 & P09429 & High mobility group protein B1 & \\
\hline HNRPK & P61978 & $\begin{array}{l}\text { Heterogeneous nuclear } \\
\text { ribonucleoprotein } \mathrm{K}\end{array}$ & Au-Kline syndrome \\
\hline IDHC & O75874 & $\begin{array}{l}\text { Isocitrate dehydrogenase [NADP] } \\
\text { cytoplasmic }\end{array}$ & Glioma \\
\hline IDHP & P48735 & $\begin{array}{l}\text { Isocitrate dehydrogenase [NADP], } \\
\text { mitochondrial }\end{array}$ & $\begin{array}{l}\text { D-2-hydroxyglutaric } \\
\text { aciduria } 2\end{array}$ \\
\hline IGLL5 & B9A064 & $\begin{array}{l}\text { Immunoglobulin lambda-like polypeptide } \\
5\end{array}$ & \\
\hline ILF2 & Q12905 & Interleukin enhancer-binding factor 2 & \\
\hline KAD3 & Q9UIJ7 & $\begin{array}{l}\text { GTP: AMP phosphotransferase AK3, } \\
\text { mitochondrial }\end{array}$ & $\begin{array}{l}\text { Phospholysine } \\
\text { phosphohistidine } \\
\text { inorganic pyrophos- } \\
\text { phate phosphatase }\end{array}$ \\
\hline LHPP & Q9H008 & & \\
\hline LKHA4 & P09960 & Leukotriene A-4 hydrolase & \\
\hline MPI & P34949 & Mannose-6-phosphate isomerase & $\begin{array}{l}\text { Congenital disorder } \\
\text { of glycosylation 1B }\end{array}$ \\
\hline
\end{tabular}




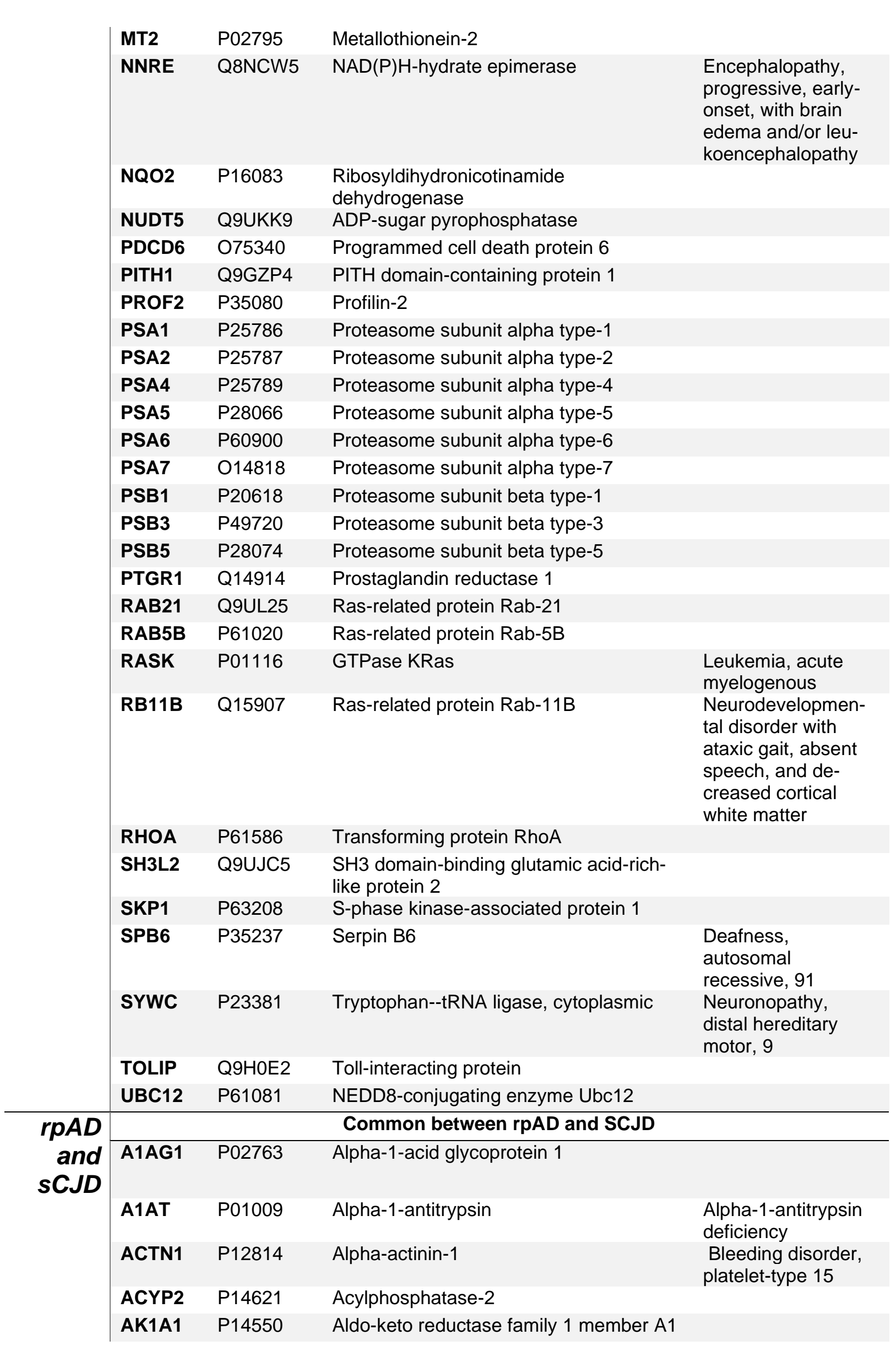




\begin{tabular}{|c|c|c|c|}
\hline CD81 & P60033 & CD81 antigen & $\begin{array}{l}\text { Immunodeficiency, } \\
\text { common variable, } 6\end{array}$ \\
\hline CYTB & P04080 & Cystatin-B & $\begin{array}{l}\text { Epilepsy, } \\
\text { progressive } \\
\text { myoclonic } 1\end{array}$ \\
\hline DNJC5 & Q9H3Z4 & DnaJ homolog subfamily $\mathrm{C}$ member 5 & $\begin{array}{l}\text { Ceroid } \\
\text { lipofuscinosis, } \\
\text { neuronal, 4B }\end{array}$ \\
\hline FRIL & P02792 & Ferritin light chain & $\begin{array}{l}\text { Hyperferritinemia } \\
\text { with or without cata- } \\
\text { ract }\end{array}$ \\
\hline FSCN1 & Q16658 & Fascin & \\
\hline GLTP & Q9NZD2 & Glycolipid transfer protein & \\
\hline GSTM2 & P28161 & Glutathione S-transferase Mu 2 & \\
\hline HPLN1 & P10915 & $\begin{array}{l}\text { Hyaluronan and proteoglycan link protein } \\
1\end{array}$ & \\
\hline LIS1 & P43034 & $\begin{array}{l}\text { Platelet-activating factor acetylhydrolase } \\
\text { IB subunit alpha }\end{array}$ & Lissencephaly 1 \\
\hline OTUB1 & Q96FW1 & Ubiquitin thioesterase OTUB1 & \\
\hline PDIA3 & P30101 & Protein disulfide-isomerase A3 & \\
\hline PEA15 & Q15121 & Astrocytic phosphoprotein PEA-15 & \\
\hline PIPNA & Q00169 & Phosphatidylinositol transfer protein alpha & soform \\
\hline PPIA & P62937 & Peptidyl-prolyl cis-trans isomerase A & \\
\hline PTGDS & P41222 & Prostaglandin-H2 D-isomerase & \\
\hline QOR & Q08257 & Quinone oxidoreductase & \\
\hline RAB5C & P51148 & Ras-related protein Rab-5C & \\
\hline RIDA & P52758 & 2-iminobutanoate/2-iminopropanoate deam & inase \\
\hline SCRN1 & Q12765 & Secernin-1 & \\
\hline SERC & Q9Y617 & Phosphoserine aminotransferase & $\begin{array}{l}\text { Phosphoserine } \\
\text { aminotransferase } \\
\text { deficiency }\end{array}$ \\
\hline SFPQ & P23246 & $\begin{array}{l}\text { Splicing factor, proline- and glutamine- } \\
\text { rich }\end{array}$ & \\
\hline SH3L1 & O75368 & $\begin{array}{l}\text { SH3 domain-binding glutamic acid-rich- } \\
\text { like protein }\end{array}$ & \\
\hline SNAA & P54920 & Alpha-soluble NSF attachment protein & \\
\hline SNAB & Q9H115 & Beta-soluble NSF attachment protein & \\
\hline SODM & P04179 & $\begin{array}{l}\text { Superoxide dismutase [Mn], } \\
\text { mitochondrial }\end{array}$ & $\begin{array}{l}\text { Microvascular } \\
\text { complications of } \\
\text { diabetes } 6\end{array}$ \\
\hline TKT & P29401 & Transketolase & $\begin{array}{l}\text { Short stature, de- } \\
\text { velopmental delay, } \\
\text { and congenital heart } \\
\text { defects }\end{array}$ \\
\hline TRFE & P02787 & Serotransferrin & \\
\hline UGPA & Q16851 & $\begin{array}{l}\text { UTP--glucose-1-phosphate } \\
\text { uridylyltransferase }\end{array}$ & \\
\hline VATH & Q9UI12 & V-type proton ATPase subunit H & \\
\hline VCP & P55072 & Valosin containing protein & $\begin{array}{l}\text { Inclusion body myo- } \\
\text { pathy with early- } \\
\text { onset Paget disease } \\
\text { with or without fron- } \\
\text { totemporal dementia } \\
1\end{array}$ \\
\hline
\end{tabular}




\begin{tabular}{|c|c|c|c|c|}
\hline \multirow{21}{*}{$\begin{array}{r}\text { spAD } \\
\text { and } \\
\text { sCJD }\end{array}$} & \multicolumn{4}{|c|}{ Common between spAD and sCJD } \\
\hline & & O43488 & \multicolumn{2}{|l|}{$\begin{array}{l}\text { Aflatoxin B1 aldehyde reductase member } \\
2\end{array}$} \\
\hline & CYBP & Q9HB71 & \multicolumn{2}{|l|}{ Calcyclin-binding protein } \\
\hline & DDAH2 & 095865 & \multicolumn{2}{|l|}{$\begin{array}{l}N(G), N(G) \text {-dimethylarginine dimethyla- } \\
\text { minohydrolase } 2\end{array}$} \\
\hline & $\mathrm{ECH} 1$ & Q13011 & \multicolumn{2}{|l|}{$\begin{array}{l}\text { Delta(3,5)-Delta }(2,4) \text {-dienoyl-CoA } \\
\text { isomeras, mitochondrial }\end{array}$} \\
\hline & ENOPH & Q9UHY7 & \multicolumn{2}{|l|}{ Enolase-phosphatase E1 } \\
\hline & FPPS & P14324 & Farnesyl pyrophosphate synthase & $\begin{array}{l}\text { Porokeratosis } 9 \\
\text { multiple types } \\
\text { (POROK9) }\end{array}$ \\
\hline & GLO2 & Q16775 & \multicolumn{2}{|l|}{$\begin{array}{l}\text { Hydroxyacylglutathione hydrolase, } \\
\text { mitochondrial }\end{array}$} \\
\hline & GLOD4 & Q9HC38 & \multicolumn{2}{|l|}{ Glyoxalase domain-containing protein 4} \\
\hline & GMFB & P60983 & \multicolumn{2}{|l|}{ Glia maturation factor beta } \\
\hline & GSTM1 & P09488 & \multicolumn{2}{|l|}{ Glutathione S-transferase Mu 1} \\
\hline & GSTM3 & P21266 & \multicolumn{2}{|l|}{ Glutathione S-transferase Mu 3} \\
\hline & LGUL & Q04760 & \multicolumn{2}{|l|}{ Lactoylglutathione lyase } \\
\hline & LSAMP & Q13449 & \multicolumn{2}{|l|}{ Limbic system-associated membrane protein } \\
\hline & MK01 & P28482 & \multicolumn{2}{|l|}{ Mitogen-activated protein kinase 1} \\
\hline & NCDN & Q9UBB6 & \multicolumn{2}{|l|}{ Neurochondrin } \\
\hline & PGM2L & Q6PCE3 & \multicolumn{2}{|l|}{ Glucose 1,6-bisphosphate synthase } \\
\hline & PP2BB & P16298 & \multirow{2}{*}{\multicolumn{2}{|c|}{$\begin{array}{l}\text { Serine/threonine-protein phosphatase } 2 B \\
\text { catalytic subunit beta isoform } \\
\text { Ras-related protein Rab-2A }\end{array}$}} \\
\hline & RAB2A & P61019 & & \\
\hline & RAB5A & P20339 & \multicolumn{2}{|l|}{ Ras-related protein Rab-5A } \\
\hline & TAGL & Q01995 & \multicolumn{2}{|l|}{ Transgelin } \\
\hline \multirow{17}{*}{$\begin{array}{r}\text { spAD } \\
\text { and } \\
\text { rpAD }\end{array}$} & \multicolumn{4}{|c|}{ Common between spAD and rpAD } \\
\hline & AKA12 & Q02952 & \multicolumn{2}{|l|}{ A-kinase anchor protein 12} \\
\hline & ANXA7 & P20073 & \multicolumn{2}{|l|}{ Annexin A7 } \\
\hline & APOE & P02649 & Apolipoprotein E & $\begin{array}{l}\text { Hyperlipoproteinemi } \\
\text { a } 3\end{array}$ \\
\hline & AQP4 & P55087 & \multicolumn{2}{|l|}{ Aquaporin-4 } \\
\hline & AT2B1 & P20020 & \multicolumn{2}{|l|}{$\begin{array}{l}\text { Plasma membrane calcium-transporting } \\
\text { ATPase } 1\end{array}$} \\
\hline & AT2B2 & Q01814 & \multicolumn{2}{|l|}{$\begin{array}{l}\text { Plasma membrane calcium-transporting } \\
\text { ATPase } 2\end{array}$} \\
\hline & AT2B3 & Q16720 & $\begin{array}{l}\text { Plasma membrane calcium-transporting } \\
\text { ATPase } 3\end{array}$ & $\begin{array}{l}\text { Spinocerebellar } \\
\text { ataxia, X-linked } 1\end{array}$ \\
\hline & AT2B4 & P23634 & \multicolumn{2}{|l|}{$\begin{array}{l}\text { Plasma membrane calcium-transporting } \\
\text { ATPase } 4\end{array}$} \\
\hline & AT5F1 & P24539 & ATP synthase $F$ & \\
\hline & ATPG & P36542 & $\begin{array}{l}\text { ATP synthase subunit gamma, } \\
\text { mitochondrial }\end{array}$ & \\
\hline & BCAS1 & O75363 & Breast carcinoma-amplified sequence 1 & \\
\hline & BSN & Q9UPA5 & Protein bassoon & \\
\hline & CADM2 & Q8N3J6 & Cell adhesion molecule 2 & \\
\hline & CXA1 & P17302 & Gap junction alpha-1 protein & $\begin{array}{l}\text { Oculodentodigital } \\
\text { dysplasia }\end{array}$ \\
\hline & EAA1 & P43003 & Excitatory amino acid transporter 1 & Episodic ataxia 6 \\
\hline & EAA2 & P43004 & Excitatory amino acid transporter 2 & $\begin{array}{l}\text { Epileptic } \\
\text { encephalopathy, }\end{array}$ \\
\hline
\end{tabular}




\begin{tabular}{|c|c|c|c|}
\hline & & & early infantile, 41 \\
\hline F10A1 & P50502 & Hsc70-interacting protein & \\
\hline FIBA & P02671 & Fibrinogen alpha chain & $\begin{array}{l}\text { Congenital } \\
\text { afibrinogenemia }\end{array}$ \\
\hline HECAM & Q14CZ8 & Hepatocyte cell adhesion molecule & $\begin{array}{l}\text { Leukoencephalopa- } \\
\text { thy, megalence- } \\
\text { phalic, with subcor- } \\
\text { tical cysts, } 2 A\end{array}$ \\
\hline MAON & Q16798 & $\begin{array}{l}\text { NADP-dependent malic enzyme, mito- } \\
\text { chondrial }\end{array}$ & \\
\hline MOG & Q16653 & Myelin-oligodendrocyte glycoprotein & Narcolepsy 7 \\
\hline MT1G & P13640 & Metallothionein-1G & \\
\hline MT3 & P25713 & Metallothionein-3 & \\
\hline NCAM2 & O15394 & Neural cell adhesion molecule 2 & \\
\hline NCAN & O14594 & Neurocan core protein & \\
\hline NHRF1 & O14745 & $\begin{array}{l}\mathrm{Na}(+) / \mathrm{H}(+) \text { exchange regulatory cofac- } \\
\text { tor NHE-RF1 }\end{array}$ & $\begin{array}{l}\text { Nephrolithiasis/oste } \\
\text { oporosis, } \\
\text { hypophosphatemic, } \\
2\end{array}$ \\
\hline NPTN & Q9Y639 & Neuroplastin & \\
\hline NRCAM & Q92823 & Neuronal cell adhesion molecule & \\
\hline OXR1 & Q8N573 & Oxidation resistance protein 1 & \\
\hline PADI2 & Q9Y2J8 & Protein-arginine deiminase type-2 & \\
\hline PALM & 075781 & Paralemmin-1 & \\
\hline PDIA6 & Q15084 & Protein disulfide-isomerase A6 & \\
\hline PFKAM & P08237 & $\begin{array}{l}\text { ATP-dependent 6-phosphofructokinase, } \\
\text { muscle type }\end{array}$ & $\begin{array}{l}\text { Glycogen storage } \\
\text { disease } 7\end{array}$ \\
\hline PLCB1 & Q9NQ66 & $\begin{array}{l}\text { 1-phosphatidylinositol } 4,5 \text {-bisphosphate } \\
\text { phosphodiesterase beta-1 }\end{array}$ & $\begin{array}{l}\text { Epileptic } \\
\text { encephalopathy, } \\
\text { early infantile, } 12\end{array}$ \\
\hline PRRT2 & Q7Z6L0 & Proline-rich transmembrane protein 2 & $\begin{array}{l}\text { Episodic kinesigenic } \\
\text { dyskinesia } 1\end{array}$ \\
\hline PTMA & P06454 & Prothymosin alpha & \\
\hline PTPRZ & P23471 & $\begin{array}{l}\text { Receptor-type tyrosine-protein phospha- } \\
\text { tase zeta }\end{array}$ & \\
\hline RAB6B & Q9NRW1 & Ras-related protein Rab-6B & \\
\hline RTN1 & Q16799 & Reticulon-1 & \\
\hline RTN4 & Q9NQC3 & Reticulon-4 & \\
\hline S10AD & Q99584 & Protein S100-A13 & \\
\hline SH3L3 & Q9H299 & $\begin{array}{l}\text { SH3 domain-binding glutamic acid-rich- } \\
\text { like protein } 3\end{array}$ & \\
\hline SNG1 & O43759 & Synaptogyrin-1 & \\
\hline TENR & Q92752 & Tenascin-R & \\
\hline TYB4 & P62328 & Thymosin beta- 4 & \\
\hline VPP1 & Q93050 & $\begin{array}{l}\text { V-type proton ATPase } 116 \mathrm{kDa} \text { subunit a } \\
\text { isoform } 1\end{array}$ & \\
\hline
\end{tabular}




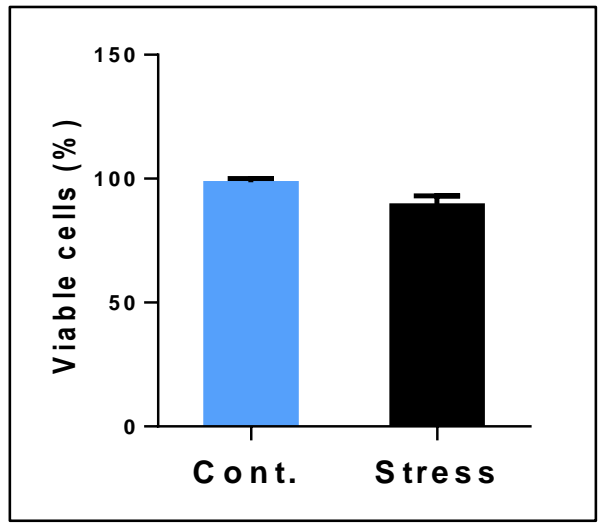

Figure 40: Cell viability assay: Trypan blue exclusion test was used to estimate the cell viability. The percentage of live cells was calculated in control (untreated) and stress cells (treated with $0.6 \mathrm{mM} \mathrm{NaAsO}$ for $60 \mathrm{~min}$ ).

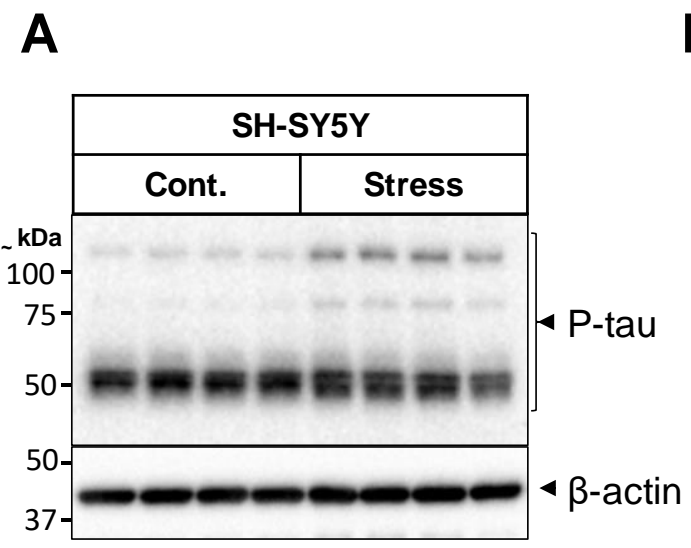

B

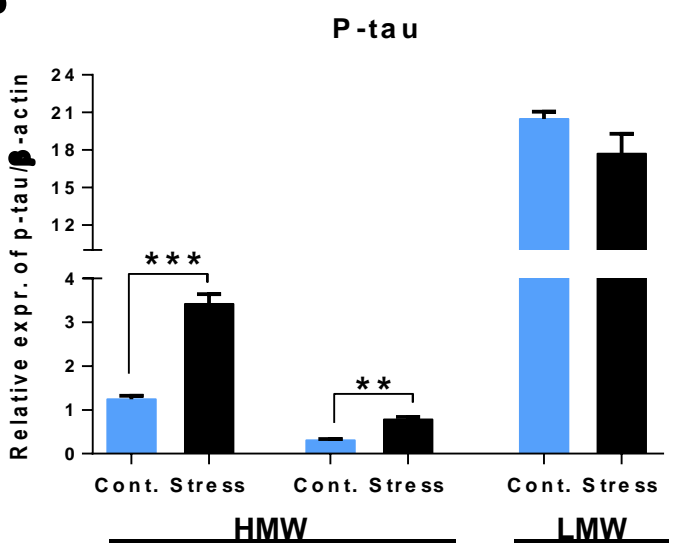

Figure 41: Stress induced increase in tau phosphorylation in SH-SY5Y cells. A) Representative immunoblots of phospho-tau in control (untreated) and stress (arsenite treated) cells. The cells were plated in 6-well plates $\left(2 \times 10^{5}\right)$ for $24 \mathrm{hrs}$ and lysed in cell-lysis buffer supplemented with protease and phosphatase inhibitors, and levels of phospho-tau were analysed by immunoblotting analysis. Intensity levels were normalized to $\beta$-actin. B) The densitometry was performed using Image Lab software. A significant increase was observed in the levels of phospho-tau in high molecular weight range (HMW $=65-250 \mathrm{kDa})$. No significant changes were observed for low molecular weight range (LMW $<65 \mathrm{kDa}$ ). Statistical tests (unpaired $t$-test) were applied in GraphPad prism, ${ }^{* *} \mathrm{p}<$ $0.01,{ }^{* \star *} \mathrm{p}<0.001$. 


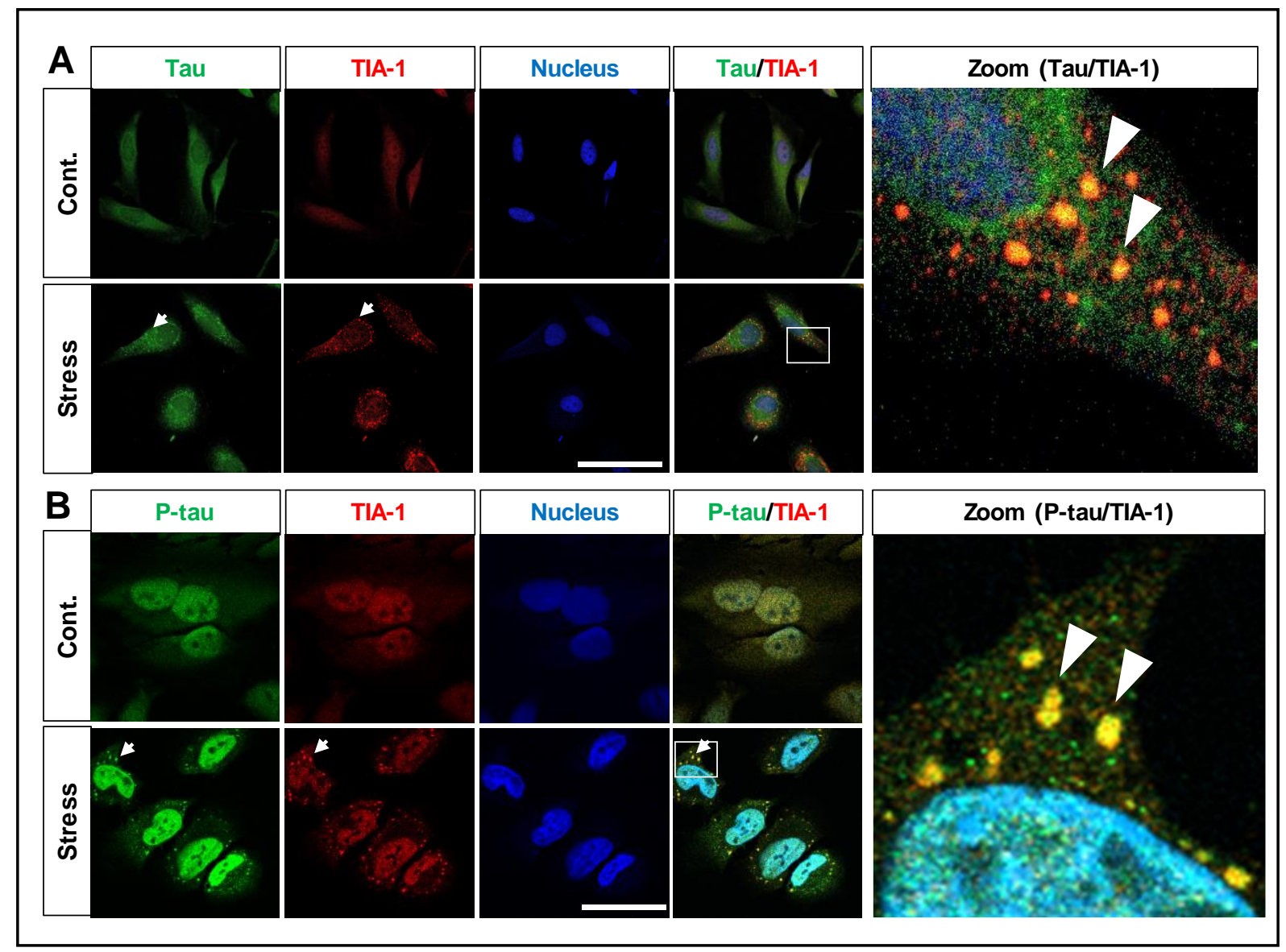

Figure 42: Tau and phospho-tau are recruited into SGs. A and B) Stress was induced with sodium arsenite and cells were co-immunostained with primary antibodies specific for total tau, phospho-tau and TIA-1, followed by incubation with AlexaFlour 488 and AlexaFlour 546 secondary antibodies. High magnification showing the expression of tau/TIA-1 and p-tau/TIA-1 for closer details in stress induced cells. Examples of SGs are indicated by the arrows, scale bar $=25 \mu \mathrm{m}$ for tau and $10 \mu \mathrm{m}$ for $\mathrm{p}$-tau.

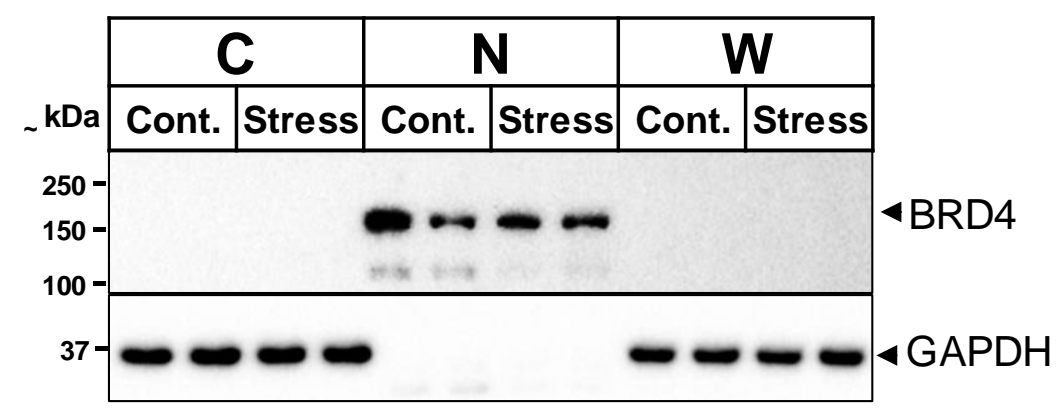

Figure 43: Subcellular fractionation after stress induction: A) Representative immunoblots for nuclear (BRD4) and cytoplasmic (GAPDH) markers in control (untreated) and stress (arsenite treated) cells, after subcellular fractionation by REAP method. Isolated fractions were abbreviated as C: cytoplasmic extract, N: nuclear extract, and W: whole cell extract. There was no cross contamination observed between nuclear and cytoplasmic markers. 


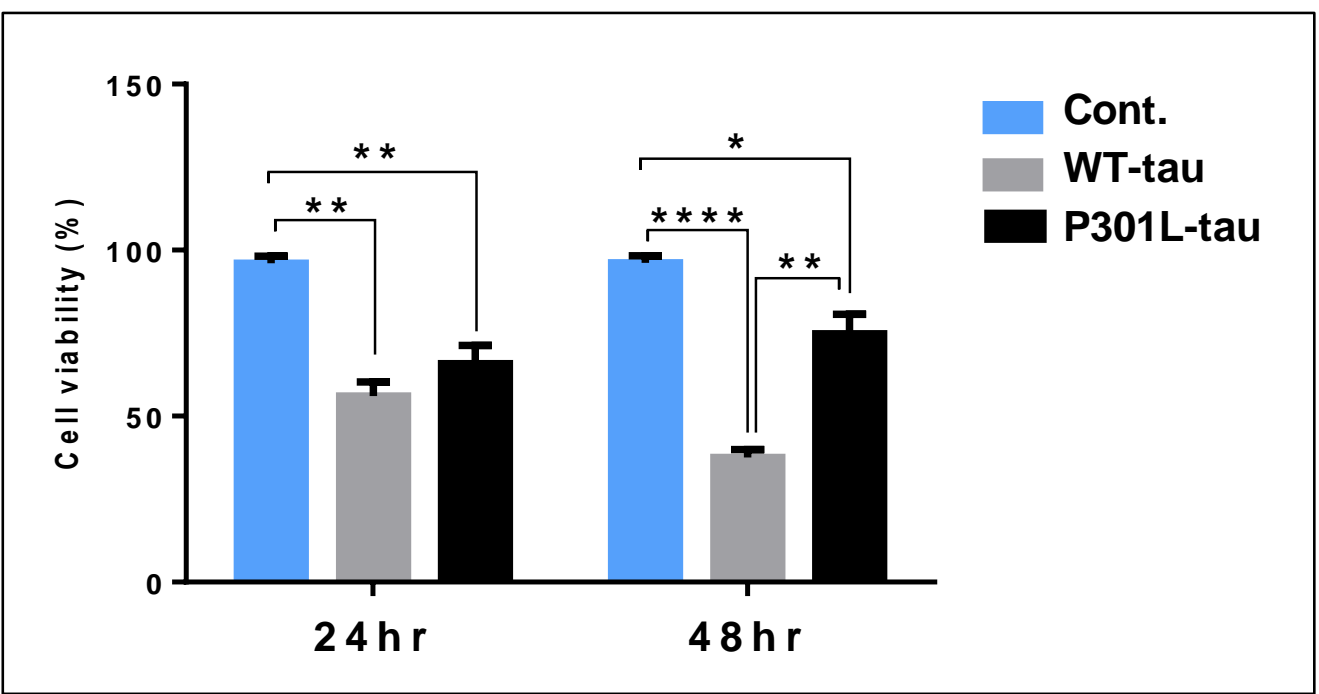

Figure 44: Cell Viability assay: The cell viability was estimated by MTS assay after expression of human-tau (both WT-tau and P301L-tau) in comparison to control at 24- and 48 hrs post-transfection. One-way ANOVA followed by Tukey post-hoc analysis was used, ${ }^{*} p<0.05,{ }^{* *} p<0.01,{ }^{* * *} p<0.001,{ }^{* * * *} p<0.0001$.

Table 16: Tau-up regulated proteins: list of unique and common proteins (with their $-\log _{10} \mathrm{p}$ values), that were upregulated after expression of either WT-tau or P301L-tau

\begin{tabular}{|c|c|c|c|c|c|}
\hline No. & UniProt ID & $\begin{array}{l}\text { UniProt } \\
\text { Accession }\end{array}$ & Protein names & $\begin{array}{c}\text { (WT vs } \\
\text { Cont.) (p- } \\
\text { values) }\end{array}$ & $\begin{array}{c}\text { (P301L vs } \\
\text { Cont.) (p- } \\
\text { values) }\end{array}$ \\
\hline 1 & IST1 & P53990 & IST1 homolog & 2.80404 & \\
\hline 2 & XCT & Q9UPY5 & Cystine/glutamate transporter & 2.70942 & \\
\hline 3 & ITPR3 & Q14573 & $\begin{array}{l}\text { Inositol 1,4,5-trisphosphate } \\
\text { receptor type } 3\end{array}$ & 3.74147 & \\
\hline 4 & CAD13 & P55290 & Cadherin-13 & 3.29636 & \\
\hline 5 & TMEDA & P49755 & $\begin{array}{l}\text { Transmembrane emp24 domain- } \\
\text { containing protein } 10\end{array}$ & 2.79315 & \\
\hline 6 & APC7 & Q9UJX3 & $\begin{array}{l}\text { Anaphase-promoting complex } \\
\text { subunit } 7\end{array}$ & 2.64649 & \\
\hline 7 & PXDC2 & Q6UX71 & $\begin{array}{l}\text { Plexin domain-containing protein } \\
2\end{array}$ & 2.60679 & \\
\hline 8 & PLCA & Q99943 & $\begin{array}{l}\text { 1-acyl-sn-glycerol-3-phosphate } \\
\text { acyltransferase alpha }\end{array}$ & 2.77202 & \\
\hline 9 & NUDC1 & Q96RS6-2 & $\begin{array}{l}\text { NudC domain-containing protein } \\
1\end{array}$ & 2.58966 & \\
\hline 10 & SYUG & O76070 & Gamma-synuclein & 3.30288 & \\
\hline 11 & $\begin{array}{l}\text { A0A1W2PS4 } \\
3\end{array}$ & $\begin{array}{l}\text { A0A1W2PS } \\
43\end{array}$ & Lysosome membrane protein 2 & 3.00432 & \\
\hline 12 & F8VX04 & F8VX04 & $\begin{array}{l}\text { Sodium-coupled neutral amino } \\
\text { acid transporter } 1\end{array}$ & 3.15582 & \\
\hline 13 & RAB18 & Q9NP72 & Ras-related protein Rab-18 & 2.60603 & \\
\hline 14 & Q5VZR0 & Q5VZR0 & $\begin{array}{l}\text { Golgi-associated plant patho- } \\
\text { genesis-related protein } 1\end{array}$ & 2.6549 & \\
\hline 15 & CD44 & P16070 & CD44 antigen & 2.89138 & \\
\hline 16 & cox2 & P00403 & Cytochrome c oxidase subunit 2 & 2.89006 & \\
\hline 17 & PYRG1 & P17812 & CTP synthase 1 & 2.64826 & \\
\hline 18 & C9JYNO & C9JYNO & Synaptophysin-like protein 1 & 3.73999 & \\
\hline 19 & E7ER44 & E7ER44 & Lactotransferrin & & 5.61449 \\
\hline
\end{tabular}




\begin{tabular}{|c|c|c|c|c|c|}
\hline 20 & K7ENL2 & K7ENL2 & \multicolumn{2}{|l|}{ WW domain-binding protein 2} & 3.25965 \\
\hline 21 & IF16 & Q16666 & \multicolumn{2}{|l|}{$\begin{array}{l}\text { Gamma-interferon-inducible } \\
\text { protein } 16\end{array}$} & 2.54071 \\
\hline 22 & $\begin{array}{l}\text { A0A1B0GW } \\
\text { C0 }\end{array}$ & $\begin{array}{l}\text { A0A1B0G } \\
\text { WC0 }\end{array}$ & \multicolumn{2}{|l|}{$\begin{array}{l}\text { Carnitine O-palmitoyltransferase } \\
2 \text {, mitochondrial }\end{array}$} & 3.83877 \\
\hline 23 & $\begin{array}{l}\text { A0A087WX9 } \\
7\end{array}$ & $\begin{array}{l}\text { A0A087WX } \\
97\end{array}$ & \multicolumn{2}{|l|}{ Bcl-2-like protein 13} & 3.19665 \\
\hline 24 & RND3 & P61587 & \multicolumn{2}{|l|}{$\begin{array}{l}\text { Rho-related GTP-binding protein } \\
\text { RhoE }\end{array}$} & 3.65369 \\
\hline 25 & PODXL & O00592 & \multicolumn{2}{|l|}{ Podocalyxin } & 5.52997 \\
\hline 26 & MOT1 & P53985 & \multicolumn{2}{|l|}{ Monocarboxylate transporter 1} & 4.65667 \\
\hline 27 & CLIC4 & Q9Y696 & \multicolumn{2}{|l|}{$\begin{array}{l}\text { Chloride intracellular channel } \\
\text { protein } 4\end{array}$} & 4.5989 \\
\hline 28 & $\begin{array}{l}\text { A0A075B73 } \\
0\end{array}$ & $\begin{array}{l}\text { A0A075B7 } \\
30\end{array}$ & \multicolumn{2}{|l|}{ Epiplakin } & 2.80249 \\
\hline 29 & S38A2 & Q96QD8 & \multicolumn{2}{|l|}{$\begin{array}{l}\text { Sodium-coupled neutral amino } \\
\text { acid transporter } 2\end{array}$} & 3.7117 \\
\hline 30 & TPBG & Q13641 & \multicolumn{2}{|l|}{ Trophoblast glycoprotein } & 3.97711 \\
\hline 31 & LAT1 & Q01650 & \multicolumn{2}{|l|}{$\begin{array}{l}\text { Large neutral amino acids trans- } \\
\text { porter small subunit } 1\end{array}$} & 6.21328 \\
\hline 32 & SQOR & Q9Y6N5 & \multicolumn{2}{|l|}{$\begin{array}{l}\text { Sulfide: quinone oxidoreductase, } \\
\text { mitochondrial }\end{array}$} & 3.5484 \\
\hline 33 & E9PEB5 & E9PEB5 & \multicolumn{2}{|l|}{$\begin{array}{l}\text { Far upstream element-binding } \\
\text { protein } 1\end{array}$} & 3.65134 \\
\hline 34 & AAAT & Q15758 & \multicolumn{2}{|l|}{ Neutral amino acid transporter B } & 4.73081 \\
\hline 35 & AHNK2 & Q8IVF2 & \multicolumn{2}{|l|}{ Protein AHNAK2 } & 2.93411 \\
\hline 36 & RTN4 & Q9NQC3-2 & \multicolumn{2}{|l|}{ Reticulon-4 } & 2.78976 \\
\hline 37 & AT1A1 & P05023-4 & \multicolumn{2}{|l|}{$\begin{array}{l}\text { Sodium/potassium-transporting } \\
\text { ATPase subunit alpha-1 }\end{array}$} & 2.80245 \\
\hline 38 & A0A087X054 & $\begin{array}{l}\text { A0A087X0 } \\
54\end{array}$ & \multicolumn{2}{|l|}{ Hypoxia up-regulated protein 1} & 2.67896 \\
\hline 39 & J3KPF3 & J3KPF3 & \multicolumn{2}{|l|}{$\begin{array}{l}\text { 4F2 cell-surface antigen heavy } \\
\text { chain }\end{array}$} & 3.40599 \\
\hline 40 & VINC & P18206 & \multicolumn{2}{|l|}{ Vinculin } & 2.67223 \\
\hline 41 & SYWC & P23381-2 & \multicolumn{2}{|l|}{$\begin{array}{l}\text { Tryptophan--tRNA ligase, } \\
\text { cytoplasmic }\end{array}$} & 2.87585 \\
\hline 42 & UAP1 & Q16222 & \multicolumn{2}{|l|}{$\begin{array}{l}\text { UDP-N-acetylhexosamine } \\
\text { pyrophosphorylase }\end{array}$} & 2.53642 \\
\hline 43 & PLST & P13797 & \multicolumn{2}{|c|}{ Plastin-3 } & 2.62095 \\
\hline \multicolumn{6}{|c|}{ Common in both WT- and P301L-tau expressing cells } \\
\hline 44 & Tau & P10636-6 & $\begin{array}{l}\text { Microtubule-associated protein } \\
\text { tau }\end{array}$ & 9.66209 & 9.99752 \\
\hline 45 & STX12 & Q86Y82 & Syntaxin-12 & 4.38935 & 3.90083 \\
\hline 46 & $\begin{array}{l}\text { AOAOAOMRJ } \\
7\end{array}$ & $\begin{array}{l}\text { AOAOAOMR } \\
\text { J7 }\end{array}$ & Coagulation factor $\mathrm{V}$ & 2.79996 & 2.55125 \\
\hline 47 & TSP1 & P07996 & Thrombospondin-1 & 4.63034 & 3.69623 \\
\hline 48 & STOM & P27105 & $\begin{array}{l}\text { Erythrocyte band } 7 \text { integral } \\
\text { membrane protein }\end{array}$ & 2.63921 & 4.70864 \\
\hline 49 & AT1B1 & P05026 & $\begin{array}{l}\text { Sodium/potassium-transporting } \\
\text { ATPase subunit beta-1 }\end{array}$ & 4.36966 & 4.04487 \\
\hline 50 & SNG2 & O43760 & Synaptogyrin-2 & 3.38169 & 3.37281 \\
\hline 51 & SODM & P04179 & $\begin{array}{l}\text { Superoxide dismutase [Mn], } \\
\text { mitochondrial }\end{array}$ & 4.35392 & 4.21758 \\
\hline 52 & E9PR17 & E9PR17 & CD59 glycoprotein & 4.06777 & 5.10992 \\
\hline 53 & MOT4 & 015427 & Monocarboxylate transporter 4 & 3.57067 & 3.15312 \\
\hline 54 & RAI3 & Q8NFJ5 & Retinoic acid-induced protein 3 & 5.11684 & 3.4051 \\
\hline
\end{tabular}




\begin{tabular}{l|lllll}
\hline 55 & RTN3 & O95197-3 & Reticulon-3 & 2.89615 & 2.83528 \\
56 & FLNB & O75369-8 & Filamin-B & 4.65931 & 4.00561 \\
57 & QCR1 & P31930 & $\begin{array}{l}\text { Cytochrome b-c1 complex sub- } \\
\text { unit 1, mitochondrial }\end{array}$ & 3.85388 & 5.56346 \\
58 & B4DKB2 & B4DKB2 & $\begin{array}{l}\text { Endothelin-converting enzyme 1 } \\
\text { Voltage-dependent anion- }\end{array}$ & 2.89353 & 2.60173 \\
59 & VDAC2 & P45880 & $\begin{array}{l}\text { Vo2634 } \\
\text { selective channel protein 2 }\end{array}$ & 3.84069 \\
60 & ANXA3 & P12429 & Annexin A3 & 2.89038 & 4.15502 \\
61 & BIP & P11021 & $\begin{array}{l}\text { Endoplasmic reticulum } \\
\text { chaperone BiP }\end{array}$ & 4.09375 & 5.35755 \\
62 & ANXA4 & P09525 & Annexin A4 & 4.25635 & 3.6306 \\
63 & A0A0G2JIW & A0A0G2JI & Heat shock 70 kDa protein 1B & 3.97859 & 2.54103 \\
& 1 & W1 & & & \\
\hline
\end{tabular}

Table 17: Tau down-regulated proteins: list of unique and common proteins (with their $-\log _{10} p$ values), that were down-regulated after expression of either WT-tau or P301L-tau

\begin{tabular}{|c|c|c|c|c|c|}
\hline No. & UniProt ID & $\begin{array}{l}\text { UniProt } \\
\text { Accession }\end{array}$ & Protein names & $\begin{array}{l}\text { WT vs } \\
\text { Cont. (p- } \\
\text { values) }\end{array}$ & $\begin{array}{l}\text { P301L vs } \\
\text { Cont. (p- } \\
\text { values) }\end{array}$ \\
\hline 1 & CAPZB & P47756-2 & $\begin{array}{l}\text { F-actin-capping protein subu- } \\
\text { nit beta }\end{array}$ & 2.90632 & \\
\hline 2 & CLIC1 & O00299 & $\begin{array}{l}\text { Chloride intracellular channel } \\
\text { protein } 1\end{array}$ & 2.74343 & \\
\hline 3 & 1433E & P62258 & 14-3-3 protein epsilon & 2.97992 & \\
\hline 4 & PDLI7 & Q9NR12 & $\begin{array}{l}\text { PDZ and LIM domain protein } \\
7\end{array}$ & 2.87187 & \\
\hline 5 & DHPR & P09417 & Dihydropteridine reductase & 3.29976 & \\
\hline 6 & TBB4B & P68371 & Tubulin beta-4B chain & 2.61161 & \\
\hline 7 & CYBP & Q9HB71 & Calcyclin-binding protein & 4.20659 & \\
\hline 8 & GSHO & P48507 & $\begin{array}{l}\text { Glutamate--cysteine ligase } \\
\text { regulatory subunit }\end{array}$ & 3.64493 & \\
\hline 9 & IDHC & O75874 & $\begin{array}{l}\text { Isocitrate dehydrogenase } \\
\text { [NADP] cytoplasmic }\end{array}$ & 3.18656 & \\
\hline 10 & CRK & P46108 & Adapter molecule crk & 2.66295 & \\
\hline 11 & LKHA4 & P09960 & Leukotriene A-4 hydrolase & 3.07686 & \\
\hline 12 & LDHA & P00338 & $\begin{array}{l}\text { L-lactate dehydrogenase A } \\
\text { chain }\end{array}$ & 2.60496 & \\
\hline 13 & COF1 & P23528 & Cofilin-1 & 2.86394 & \\
\hline 14 & PSME3 & P61289 & $\begin{array}{l}\text { Proteasome activator complex } \\
\text { subunit } 3\end{array}$ & 2.59093 & \\
\hline 15 & XPO6 & Q96QU8 & Exportin-6 & 2.63751 & \\
\hline 16 & PFKAM & P08237 & $\begin{array}{l}\text { ATP-dependent 6- } \\
\text { phosphofructokinase, muscle } \\
\text { type }\end{array}$ & 2.77411 & \\
\hline 17 & METK2 & P31153 & $\begin{array}{l}\text { S-adenosylmethionine syn- } \\
\text { thase isoform type-2 }\end{array}$ & 2.59551 & \\
\hline 18 & I3LOH8 & I3LOH8 & $\begin{array}{l}\text { ATP-dependent RNA helicase } \\
\text { DDX19A }\end{array}$ & 3.00712 & \\
\hline 19 & A0A087WYT3 & A0A087WYT3 & Prostaglandin E synthase 3 & 6.25017 & \\
\hline 20 & MBB1A & Q9BQG0 & Myb-binding protein $1 \mathrm{~A}$ & 3.31109 & \\
\hline 21 & GANAB & Q14697-2 & Neutral alpha-glucosidase $A B$ & 3.99331 & \\
\hline 22 & RPAC1 & O15160 & $\begin{array}{l}\text { DNA-directed RNA polymer- } \\
\text { ases I and III subunit RPAC1 }\end{array}$ & 2.95675 & \\
\hline 23 & 6PGD & P52209 & 6-phosphogluconate & 3.15286 & \\
\hline
\end{tabular}




\begin{tabular}{|c|c|c|c|c|}
\hline & & & $\begin{array}{l}\text { dehydrogenase, } \\
\text { decarboxylating }\end{array}$ & \\
\hline 24 & F2Z2Y4 & F2Z2Y4 & Pyridoxal kinase & 3.349 \\
\hline 25 & KTHY & P23919 & Thymidylate kinase & 4.05072 \\
\hline 26 & ACADM & P11310-2 & $\begin{array}{l}\text { Medium-chain specific acyl- } \\
\text { CoA dehydrogenase, mito- } \\
\text { chondrial }\end{array}$ & 3.37726 \\
\hline 27 & RT27 & Q92552 & $\begin{array}{l}28 \mathrm{~S} \text { ribosomal protein } \mathrm{S} 27 \text {, } \\
\text { mitochondrial }\end{array}$ & 2.65651 \\
\hline 28 & A0A1B0GW77 & A0A1B0GW77 & $\begin{array}{l}\text { Alpha-aminoadipic } \\
\text { semialdehyde dehydrogenase }\end{array}$ & 2.57993 \\
\hline 29 & IPYR & Q15181 & Inorganic pyrophosphatase & 2.56092 \\
\hline 30 & KPYM & $\mathrm{P} 14618$ & Pyruvate kinase PKM & 3.3471 \\
\hline 31 & TWF1 & Q12792 & Twinfilin-1 & 3.16219 \\
\hline 32 & MCM6 & Q14566 & $\begin{array}{l}\text { DNA replication licensing fac- } \\
\text { tor MCM6 }\end{array}$ & 2.98343 \\
\hline 33 & I3L2B0 & I3L2B0 & $\begin{array}{l}\text { Clustered mitochondria } \\
\text { protein homolog }\end{array}$ & 3.14353 \\
\hline 34 & KPRA & Q14558 & $\begin{array}{l}\text { Phosphoribosyl pyrophos- } \\
\text { phate synthase-associated } \\
\text { protein } 1\end{array}$ & 2.88641 \\
\hline 35 & ER01A & Q96HE7 & ERO1-like protein alpha & 3.48398 \\
\hline 36 & AP3D1 & 014617 & AP-3 complex subunit delta-1 & 2.64148 \\
\hline 37 & Q5QPM7 & Q5QPM7 & $\begin{array}{l}\text { Proteasome inhibitor PI31 } \\
\text { subunit }\end{array}$ & 2.779 \\
\hline 38 & TNPO3 & Q9Y5L0 & Transportin-3 & 3.19029 \\
\hline 39 & NAT10 & Q9H0A0 & $\begin{array}{l}\text { RNA cytidine } \\
\text { acetyltransferase }\end{array}$ & 2.60558 \\
\hline 40 & F6WQW2 & F6WQW2 & $\begin{array}{l}\text { Ran-specific GTPase- } \\
\text { activating protein }\end{array}$ & 2.6097 \\
\hline 41 & HAT1 & 014929 & $\begin{array}{l}\text { Histone acetyltransferase type } \\
\text { B catalytic subunit }\end{array}$ & 2.61858 \\
\hline 42 & E7ESZ7 & E7ESZ7 & $\begin{array}{l}\text { NADH dehydrogenase [ubiq- } \\
\text { uinone] } 1 \text { alpha subcomplex } \\
\text { subunit } 10, \text { mitochondrial }\end{array}$ & 3.34015 \\
\hline 43 & A0A1B0GWE8 & A0A1B0GWE8 & Cathepsin D & 3.59333 \\
\hline 44 & APEX1 & P27695 & $\begin{array}{l}\text { DNA-(apurinic or apyrimidinic } \\
\text { site) lyase }\end{array}$ & 2.573 \\
\hline 45 & LNP & Q9C0E8 & $\begin{array}{l}\text { Endoplasmic reticulum junc- } \\
\text { tion formation protein lunapark }\end{array}$ & 3.024 \\
\hline 46 & PARK7 & Q99497 & $\begin{array}{l}\text { Protein/nucleic acid deglycase } \\
\text { DJ-1 }\end{array}$ & 3.53845 \\
\hline 47 & PP2AA & P67775 & $\begin{array}{l}\text { Serine/threonine-protein } \\
\text { phosphatase } 2 \mathrm{~A} \text { catalytic sub- } \\
\text { unit alpha isoform }\end{array}$ & 2.85783 \\
\hline 48 & DUS23 & Q9BVJ7 & $\begin{array}{l}\text { Dual specificity protein } \\
\text { phosphatase } 23\end{array}$ & 2.6001 \\
\hline 49 & NUP43 & Q8NFH3 & Nucleoporin Nup43 & 2.92109 \\
\hline 50 & TF3C4 & Q9UKN8 & $\begin{array}{l}\text { General transcription factor } \\
\text { 3C polypeptide } 4\end{array}$ & 2.85149 \\
\hline 51 & UBA3 & Q8TBC4 & $\begin{array}{l}\text { NEDD8-activating enzyme E1 } \\
\text { catalytic subunit }\end{array}$ & 2.85788 \\
\hline 52 & B1AH49 & $\mathrm{B} 1 \mathrm{AH} 49$ & $\begin{array}{l}\text { 3-mercaptopyruvate } \\
\text { sulfurtransferase }\end{array}$ & 4.16312 \\
\hline 53 & ABCD3 & P28288 & $\begin{array}{l}\text { ATP-binding cassette sub- } \\
\text { family D member } 3\end{array}$ & 2.89151 \\
\hline 54 & Q5QPR3 & Q5QPR3 & Cyclin-dependent kinase $11 \mathrm{~A}$ & 3.07425 \\
\hline
\end{tabular}




\begin{tabular}{|c|c|c|c|c|c|}
\hline 55 & D6RG13 & D6RG13 & $40 \mathrm{~S}$ ribosomal protein $\mathrm{S} 3 \mathrm{a}$ & 3.40351 & \\
\hline 56 & B7Z4B8 & B7Z4B8 & $\begin{array}{l}\text { Heterogeneous nuclear ribo- } \\
\text { nucleoprotein U-like protein } 1\end{array}$ & 3.80247 & \\
\hline 57 & AN32E & Q9BTT0 & $\begin{array}{l}\text { Acidic leucine-rich nuclear } \\
\text { phosphoprotein } 32 \text { family } \\
\text { member E }\end{array}$ & 3.0431 & \\
\hline 58 & HPBP1 & Q9NZL4 & Hsp70-binding protein 1 & 3.14118 & \\
\hline 59 & PAIRB & Q8NC51 & $\begin{array}{l}\text { Plasminogen activator } \\
\text { inhibitor } 1 \text { RNA-binding } \\
\text { protein }\end{array}$ & 2.72168 & \\
\hline 60 & CDK1 & P06493 & Cyclin-dependent kinase 1 & 6.03772 & \\
\hline 61 & X6RLT1 & X6RLT1 & $\begin{array}{l}\text { Negative elongation factor } \\
C / D\end{array}$ & 2.93622 & \\
\hline 62 & TNAP2 & Q03169 & $\begin{array}{l}\text { Tumor necrosis factor alpha- } \\
\text { induced protein } 2\end{array}$ & 2.62938 & \\
\hline 63 & MLKL & Q8NB16 & $\begin{array}{l}\text { Mixed lineage kinase domain- } \\
\text { like protein }\end{array}$ & 3.39344 & \\
\hline 64 & E7ETKO & E7ETK0 & 40S ribosomal protein S24 & 3.59647 & \\
\hline 65 & DHX36 & Q9H2U1 & $\begin{array}{l}\text { ATP-dependent DNA/RNA } \\
\text { helicase DHX36 }\end{array}$ & 3.02912 & \\
\hline 66 & TBCC & Q15814 & Tubulin-specific chaperone C & 2.65875 & \\
\hline 67 & RL6 & Q02878 & $60 S$ ribosomal protein L6 & 3.71789 & \\
\hline 68 & TOM34 & Q15785 & $\begin{array}{l}\text { Mitochondrial import receptor } \\
\text { subunit TOM34 }\end{array}$ & 3.64649 & \\
\hline 69 & HP1B3 & Q5SSJ5 & $\begin{array}{l}\text { Heterochromatin protein } 1 \text { - } \\
\text { binding protein } 3\end{array}$ & 3.88668 & \\
\hline 70 & C19L1 & Q69YN2 & CWF19-like protein 1 & 2.65963 & \\
\hline 71 & RS17 & P08708 & $40 S$ ribosomal protein $\mathrm{S} 17$ & 3.74528 & \\
\hline 72 & E7ESA6 & E7ESA6 & Focal adhesion kinase 1 & 2.64713 & \\
\hline 73 & AP3M1 & Q9Y2T2 & AP-3 complex subunit mu-1 & 2.75333 & \\
\hline 74 & M0QXD6 & M0QXD6 & $\begin{array}{l}\text { General transcription factor IIF } \\
\text { subunit } 1\end{array}$ & 2.56235 & \\
\hline 75 & WNT5A & P41221 & Protein Wnt-5a & 2.59575 & \\
\hline 76 & GMDS & 060547 & $\begin{array}{l}\text { GDP-mannose } 4,6 \\
\text { dehydratase }\end{array}$ & 3.08654 & \\
\hline 77 & I3L0X5 & I3L0X5 & Sperm-associated antigen 7 & 3.3113 & \\
\hline 78 & E9PH64 & E9PH64 & $\begin{array}{l}\text { NADH dehydrogenase [ubiq- } \\
\text { uinone] } 1 \text { beta subcomplex } \\
\text { subunit } 9\end{array}$ & 2.95705 & \\
\hline 79 & MIPEP & Q99797 & $\begin{array}{l}\text { Mitochondrial intermediate } \\
\text { peptidase }\end{array}$ & 2.7807 & \\
\hline 80 & A0A087WZR9 & A0A087WZR9 & $\begin{array}{l}\text { Pyrroline-5-carboxylate } \\
\text { reductase }\end{array}$ & 5.26058 & \\
\hline 81 & CHIP & Q9UNE7 & $\begin{array}{l}\text { E3 ubiquitin-protein ligase } \\
\text { CHIP }\end{array}$ & 2.93915 & \\
\hline 82 & TBCE & Q15813 & Tubulin-specific chaperone E & 2.57258 & \\
\hline 83 & SAAL1 & Q96ER3 & Protein SAAL1 & 2.84296 & \\
\hline 84 & SYRC & P54136 & $\begin{array}{l}\text { Arginine--tRNA ligase, } \\
\text { cytoplasmic }\end{array}$ & & 3.68764 \\
\hline 85 & HTAl2 & Q9BUP3 & Oxidoreductase HTATIP2 & & 2.52883 \\
\hline 86 & GRP75 & P38646 & $\begin{array}{l}\text { Stress- } 70 \text { protein, } \\
\text { mitochondrial }\end{array}$ & & 3.35915 \\
\hline 87 & PSB3 & P49720 & $\begin{array}{l}\text { Proteasome subunit beta } \\
\text { type-3 }\end{array}$ & & 2.82347 \\
\hline 88 & GSTP1 & P09211 & Glutathione S-transferase P & & 3.25767 \\
\hline 89 & RAB7A & P51149 & Ras-related protein Rab-7a & & 2.95429 \\
\hline
\end{tabular}




\begin{tabular}{|c|c|c|c|c|}
\hline 90 & TCPG & P49368 & $\begin{array}{l}\text { T-complex protein } 1 \text { subunit } \\
\text { gamma }\end{array}$ & 3.5822 \\
\hline 91 & RAB9A & P51151 & Ras-related protein Rab-9A & 2.60002 \\
\hline 92 & PHB & P35232 & Prohibitin & 2.76765 \\
\hline 93 & RS27A & P62979 & $\begin{array}{l}\text { Ubiquitin-40S ribosomal pro- } \\
\text { tein S27a }\end{array}$ & 3.44529 \\
\hline 94 & E7EQR4 & E7EQR4 & Ezrin & 2.70739 \\
\hline 95 & RIR1 & P23921 & $\begin{array}{l}\text { Ribonucleoside-diphosphate reductase } \\
\text { large subunit }\end{array}$ & 2.55098 \\
\hline 96 & J3QQT2 & J3QQT2 & 605 ribosomal protein L17 & 2.99247 \\
\hline 97 & SRP68 & Q9UHB9 & $\begin{array}{l}\text { Signal recognition particle } \\
\text { subunit SRP68 }\end{array}$ & 3.01785 \\
\hline 98 & SMD3 & P62318 & $\begin{array}{l}\text { Small nuclear ribonucleopro- } \\
\text { tein Sm D3 }\end{array}$ & 3.12363 \\
\hline 99 & SYQ & P47897 & Glutamine--tRNA ligase & 2.65577 \\
\hline 100 & H3BQI1 & H3BQI1 & $\begin{array}{l}\text { Dynein light chain roadblock- } \\
\text { type } 2\end{array}$ & 2.72107 \\
\hline 101 & MCM5 & P33992 & $\begin{array}{l}\text { DNA replication licensing fac- } \\
\text { tor MCM5 }\end{array}$ & 2.94611 \\
\hline 102 & Q8WVC2 & Q8WVC2 & $40 S$ ribosomal protein $\mathrm{S} 21$ & 2.76124 \\
\hline 103 & NDUS3 & O75489 & $\begin{array}{l}\text { NADH dehydrogenase [ubiquinone] iron- } \\
\text { sulfur protein } 3 \text {, mitochondrial }\end{array}$ & 3.63337 \\
\hline 104 & RL7 & P18124 & $60 S$ ribosomal protein $\mathrm{L} 7$ & 2.73981 \\
\hline 105 & C9JA28 & C9JA28 & $\begin{array}{l}\text { Translocon-associated protein } \\
\text { subunit gamma }\end{array}$ & 3.0665 \\
\hline 106 & S4R3E9 & S4R3E9 & NEDD8-MDP1 readthrough & 3.09505 \\
\hline 107 & VIME & P08670 & Vimentin & 3.24861 \\
\hline 108 & I3L504 & I3L504 & $\begin{array}{l}\text { Eukaryotic translation initiation } \\
\text { factor } 5 \mathrm{~A}-1\end{array}$ & 2.65408 \\
\hline 109 & TM109 & Q9BVC6 & Transmembrane protein 109 & 3.52926 \\
\hline 110 & DRG1 & Q9Y295 & $\begin{array}{l}\text { Developmentally-regulated GTP-binding } \\
\text { protein } 1\end{array}$ & 3.14037 \\
\hline 111 & SMD1 & P62314 & $\begin{array}{l}\text { Small nuclear ribonucleopro- } \\
\text { tein Sm D1 }\end{array}$ & 3.54617 \\
\hline 112 & HOYEN5 & HOYEN5 & $40 S$ ribosomal protein $S 2$ & 3.76272 \\
\hline 113 & MCM3 & P25205-2 & $\begin{array}{l}\text { DNA replication licensing fac- } \\
\text { tor } \mathrm{MCM} 3\end{array}$ & 3.01339 \\
\hline 114 & ARF6 & P62330 & ADP-ribosylation factor 6 & 3.18205 \\
\hline 115 & RL7A & P62424 & $60 S$ ribosomal protein $\mathrm{L} 7 \mathrm{a}$ & 3.56152 \\
\hline 116 & BZW2 & Q9Y6E2 & $\begin{array}{l}\text { Basic leucine zipper and W2 domain- } \\
\text { containing protein } 2\end{array}$ & 4.20893 \\
\hline 117 & AOAOAOMTNO & AOAOAOMTNO & Cullin-2 & 2.85465 \\
\hline 118 & THOP1 & P52888 & Thimet oligopeptidase & 3.49956 \\
\hline 119 & J3QRI7 & J3QRI7 & $60 S$ ribosomal protein L26 & 5.03306 \\
\hline 120 & ATPO & P48047 & $\begin{array}{l}\text { ATP synthase subunit } \mathrm{O}, \mathrm{mi}- \\
\text { tochondrial }\end{array}$ & 2.73623 \\
\hline 121 & LSM3 & P62310 & $\begin{array}{l}\text { U6 snRNA-associated Sm-like } \\
\text { protein LSm3 }\end{array}$ & 3.12073 \\
\hline 122 & SRP14 & P37108 & $\begin{array}{l}\text { Signal recognition particle } 14 \\
\text { kDa protein }\end{array}$ & 2.97832 \\
\hline 123 & NCBP2 & P52298 & $\begin{array}{l}\text { Nuclear cap-binding protein } \\
\text { subunit } 2\end{array}$ & 2.55743 \\
\hline 124 & RAN & P62826 & $\begin{array}{l}\text { GTP-binding nuclear protein } \\
\text { Ran }\end{array}$ & 2.58877 \\
\hline 125 & J3KQ48 & J3KQ48 & $\begin{array}{l}\text { Peptidyl-tRNA hydrolase } 2 \text {, } \\
\text { mitochondrial }\end{array}$ & 4.00593 \\
\hline
\end{tabular}




\begin{tabular}{|c|c|c|c|}
\hline TXD17 & Q9BRA2 & $\begin{array}{l}\text { Thioredoxin domain- } \\
\text { containing protein } 17\end{array}$ & 3.25391 \\
\hline MGST1 & P10620 & $\begin{array}{l}\text { Microsomal glutathione S- } \\
\text { transferase } 1\end{array}$ & 2.82746 \\
\hline SNX1 & Q13596 & Sorting nexin-1 & 2.5274 \\
\hline HNRPF & P52597 & $\begin{array}{l}\text { Heterogeneous nuclear } \\
\text { ribonucleoprotein } \mathrm{F}\end{array}$ & 2.89938 \\
\hline RL13 & P26373 & $60 S$ ribosomal protein L13 & 3.9096 \\
\hline A0A087WUD3 & A0A087WUD3 & $\begin{array}{l}\text { Oligosaccharyltransferase complex subunit } \\
\text { OSTC }\end{array}$ & 2.65514 \\
\hline RS28 & P62857 & $40 S$ ribosomal protein S28 & 5.78257 \\
\hline J3KTA4 & J3KTA4 & $\begin{array}{l}\text { Probable ATP-dependent } \\
\text { RNA helicase DDX5 }\end{array}$ & 4.43944 \\
\hline DDX6 & P26196 & $\begin{array}{l}\text { Probable ATP-dependent } \\
\text { RNA helicase DDX6 }\end{array}$ & 2.70974 \\
\hline ZC3HF & Q8WU90 & $\begin{array}{l}\text { Zinc finger } \mathrm{CCCH} \text { domain-containing pro- } \\
\text { tein } 15\end{array}$ & 2.64774 \\
\hline RM21 & Q7Z2W9 & $\begin{array}{l}39 S \text { ribosomal protein } \mathrm{L} 21 \\
\text { mitochondrial }\end{array}$ & 3.18026 \\
\hline ULA1 & Q13564 & $\begin{array}{l}\text { NEDD8-activating enzyme E1 regulatory } \\
\text { subunit }\end{array}$ & 3.12154 \\
\hline RL11 & P62913 & $60 S$ ribosomal protein L11 & 4.33191 \\
\hline RS11 & P62280 & $40 S$ ribosomal protein $\mathrm{S} 11$ & 3.57281 \\
\hline RS8 & P62241 & $40 S$ ribosomal protein $S 8$ & 4.43922 \\
\hline RL21 & P46778 & $60 S$ ribosomal protein L21 & 3.30215 \\
\hline A0A087X0X3 & A0A087X0X3 & $\begin{array}{l}\text { Heterogeneous nuclear } \\
\text { ribonucleoprotein M }\end{array}$ & 2.92309 \\
\hline RS23 & P62266 & $40 S$ ribosomal protein S23 & 4.45084 \\
\hline ATD3A & Q9NVI7-2 & $\begin{array}{l}\text { ATPase family AAA domain-containing } \\
\text { protein } 3 A\end{array}$ & 2.65768 \\
\hline RL23 & P62829 & $60 S$ ribosomal protein L23 & 4.39359 \\
\hline 2A5D & Q14738-3 & $\begin{array}{l}\text { Serine/threonine-protein phosphatase } 2 \mathrm{~A} \\
56 \mathrm{kDa} \text { regulatory subunit delta isoform }\end{array}$ & 3.24429 \\
\hline RS13 & P62277 & $40 S$ ribosomal protein $\mathrm{S} 13$ & 5.13601 \\
\hline G5E9Q6 & G5E9Q6 & Profilin & 3.91099 \\
\hline RL12 & P30050 & $60 S$ ribosomal protein $L 12$ & 4.5988 \\
\hline XPO5 & Q9HAV4 & Exportin-5 & 2.81304 \\
\hline DDX23 & Q9BUQ8 & $\begin{array}{l}\text { Probable ATP-dependent RNA helicase } \\
\text { DDX23 }\end{array}$ & 3.09004 \\
\hline RL27 & P61353 & $60 S$ ribosomal protein L27 & 5.56322 \\
\hline E5RI99 & E5RI99 & $60 S$ ribosomal protein L30 & 4.79294 \\
\hline SHIP2 & O15357 & $\begin{array}{l}\text { Phosphatidylinositol 3,4,5-trisphosphate 5- } \\
\text { phosphatase } 2\end{array}$ & 2.93228 \\
\hline PRP6 & O94906 & $\begin{array}{l}\text { Pre-mRNA-processing factor } \\
6\end{array}$ & 4.71226 \\
\hline SYDM & Q6PI48 & $\begin{array}{l}\text { Aspartate--tRNA ligase, } \\
\text { mitochondrial }\end{array}$ & 4.19994 \\
\hline KIF2A & O00139-2 & Kinesin-like protein KIF2A & 2.70644 \\
\hline J3QR09 & J3QR09 & Ribosomal protein L19 & 2.74218 \\
\hline OCAD2 & Q56VL3 & $\begin{array}{l}\text { OCIA domain-containing } \\
\text { protein } 2\end{array}$ & 4.79686 \\
\hline J3QSV6 & J3QSV6 & $\begin{array}{l}\text { Ribosomal L1 domain- } \\
\text { containing protein } 1\end{array}$ & 4.28401 \\
\hline RL38 & P63173 & $60 S$ ribosomal protein L38 & 2.93381 \\
\hline A0A087X2D0 & A0A087X2D0 & $\begin{array}{l}\text { Serine/arginine-rich-splicing } \\
\text { factor } 3\end{array}$ & 4.36894 \\
\hline
\end{tabular}




\begin{tabular}{|c|c|c|c|c|c|}
\hline 163 & GPI8 & Q92643 & \multicolumn{2}{|l|}{ GPI-anchor transamidase } & 2.86273 \\
\hline 164 & NU160 & Q12769 & \multicolumn{2}{|l|}{$\begin{array}{l}\text { Nuclear pore complex protein } \\
\text { Nup160 }\end{array}$} & 2.76185 \\
\hline 165 & SMYD3 & Q9H7B4 & \multicolumn{2}{|l|}{$\begin{array}{l}\text { Histone-lysine } \mathrm{N} \text { - } \\
\text { methyltransferase SMYD3 }\end{array}$} & 3.32229 \\
\hline 166 & YMEL1 & Q96TA2 & \multicolumn{2}{|l|}{$\begin{array}{l}\text { ATP-dependent zinc metallo- } \\
\text { protease YME1L1 }\end{array}$} & 2.84011 \\
\hline 167 & PDCD4 & Q53EL6 & \multicolumn{2}{|l|}{$\begin{array}{l}\text { Programmed cell death } \\
\text { protein } 4\end{array}$} & 3.59557 \\
\hline 168 & TMED1 & Q13445 & \multicolumn{2}{|c|}{$\begin{array}{l}\text { Transmembrane emp24 domain-containing } \\
\text { protein } 1\end{array}$} & 2.72926 \\
\hline 169 & RCN2 & Q14257 & \multicolumn{2}{|c|}{ Reticulocalbin-2 } & 3.22922 \\
\hline 170 & R39L5 & Q59GN2 & \multicolumn{2}{|l|}{$\begin{array}{l}\text { Putative } 60 \text { S ribosomal pro- } \\
\text { tein L39-like } 5\end{array}$} & 5.63947 \\
\hline 171 & SRP09 & P49458 & \multicolumn{2}{|l|}{$\begin{array}{l}\text { Signal recognition particle } 9 \\
\text { kDa protein }\end{array}$} & 4.54385 \\
\hline 172 & TSR1 & Q2NL82 & \multicolumn{2}{|l|}{$\begin{array}{l}\text { Pre-rRNA-processing protein } \\
\text { TSR1 homolog }\end{array}$} & 2.96676 \\
\hline 173 & DYR & P00374 & \multicolumn{2}{|l|}{ Dihydrofolate reductase } & 4.23334 \\
\hline 174 & DHX8 & Q14562 & \multicolumn{2}{|l|}{$\begin{array}{l}\text { ATP-dependent RNA helicase } \\
\text { DHX8 }\end{array}$} & 2.56375 \\
\hline 175 & RIPK1 & Q13546 & \multicolumn{2}{|c|}{$\begin{array}{l}\text { Receptor-interacting serine/threonine- } \\
\text { protein kinase } 1\end{array}$} & 3.70312 \\
\hline \multicolumn{6}{|c|}{ Common in both WT- and P301L- tau expressing cells } \\
\hline 176 & F8WCF6 & F8WCF6 & $\begin{array}{l}\text { Actin-related protein } 2 / 3 \text { com- } \\
\text { plex subunit } 4\end{array}$ & 3.33715 & 4.14691 \\
\hline 177 & RACK1 & P63244 & $\begin{array}{l}\text { Receptor of activated protein } \\
\text { C kinase } 1\end{array}$ & 3.39824 & 4.51018 \\
\hline 178 & SYDC & P14868 & $\begin{array}{l}\text { Aspartate--tRNA ligase, } \\
\text { cytoplasmic }\end{array}$ & 2.74959 & 2.57307 \\
\hline 179 & EIF3K & Q9UBQ5 & $\begin{array}{l}\text { Eukaryotic translation initiation } \\
\text { factor } 3 \text { subunit } \mathrm{K}\end{array}$ & 2.81828 & 4.58194 \\
\hline 180 & H9KV45 & H9KV45 & $\begin{array}{l}\text { Ubiquitin-conjugating enzyme } \\
\text { E2 D3 }\end{array}$ & 2.67823 & 5.42209 \\
\hline 181 & Q5JR08 & Q5JR08 & $\begin{array}{l}\text { Rho-related GTP-binding pro- } \\
\text { tein RhoC }\end{array}$ & 3.44748 & 3.81268 \\
\hline 182 & PROF1 & P07737 & Profilin-1 & 3.07127 & 4.69295 \\
\hline 183 & FPPS & P14324 & $\begin{array}{l}\text { Farnesyl pyrophosphate } \\
\text { synthase }\end{array}$ & 3.80014 & 3.24431 \\
\hline 184 & Q5VV89 & Q5VV89 & $\begin{array}{l}\text { Microsomal glutathione S- } \\
\text { transferase } 3\end{array}$ & 3.6279 & 4.42465 \\
\hline 185 & SYK & Q15046 & Lysine--tRNA ligase & 3.9661 & 3.1659 \\
\hline 186 & PUR2 & P22102 & $\begin{array}{l}\text { Trifunctional purine biosyn- } \\
\text { thetic protein adenosine-3 } \\
\text { [Includes: Phosphoribosyla- } \\
\text { mine--glycine ligase }\end{array}$ & 2.81901 & 2.93211 \\
\hline 187 & SND1 & Q7KZF4 & $\begin{array}{l}\text { Staphylococcal nuclease do- } \\
\text { main-containing protein } 1\end{array}$ & 3.019 & 3.608 \\
\hline 188 & C9JZR2 & C9JZR2 & Catenin delta-1 & 2.6207 & 2.99525 \\
\hline 189 & CAN2 & P17655 & Calpain-2 catalytic subunit & 3.42537 & 3.11891 \\
\hline 190 & AAAS & Q9NRG9 & Aladin & 4.28764 & 4.41039 \\
\hline 191 & SYLC & Q9P2J5 & $\begin{array}{l}\text { Leucine--tRNA ligase, } \\
\text { cytoplasmic }\end{array}$ & 3.39758 & 3.6911 \\
\hline 192 & RNZ2 & Q9BQ52 & $\begin{array}{l}\text { Zinc phosphodiesterase ELAC } \\
\text { protein } 2\end{array}$ & 3.34161 & 3.22 \\
\hline 193 & TIF1B & Q13263 & $\begin{array}{l}\text { Transcription intermediary } \\
\text { factor } 1 \text {-beta }\end{array}$ & 3.23108 & 3.44094 \\
\hline 194 & EIF3B & P55884 & Eukaryotic translation initiation & 4.32237 & 4.51386 \\
\hline
\end{tabular}




\begin{tabular}{|c|c|c|c|c|c|}
\hline & & & factor 3 subunit B & & \\
\hline 195 & PPIA & P62937 & $\begin{array}{l}\text { Peptidyl-prolyl cis-trans iso- } \\
\text { merase A }\end{array}$ & 3.8054 & 4.17 \\
\hline 196 & HOYAK1 & HOYAK1 & G-rich sequence factor 1 & 3.46677 & 2.53854 \\
\hline 197 & EIF3G & O75821 & $\begin{array}{l}\text { Eukaryotic translation initiation } \\
\text { factor } 3 \text { subunit } G\end{array}$ & 2.72457 & 3.04545 \\
\hline 198 & Q32Q12 & Q32Q12 & $\begin{array}{l}\text { Nucleoside diphosphate } \\
\text { kinase }\end{array}$ & 3.00854 & 3.04007 \\
\hline 199 & EFTU & P49411 & $\begin{array}{l}\text { Elongation factor Tu, } \\
\text { mitochondrial }\end{array}$ & 3.64175 & 3.42478 \\
\hline 200 & RS3 & P23396 & 40 S ribosomal protein S3 & 2.61813 & 3.38603 \\
\hline 201 & MTREX & P42285 & Exosome RNA helicase MTR4 & 2.57741 & 2.61761 \\
\hline 202 & RS26 & P62854 & 40 S ribosomal protein S26 & 2.66498 & 3.15958 \\
\hline 203 & RS16 & P62249 & $40 S$ ribosomal protein $\mathrm{S} 16$ & 2.87042 & 5.66172 \\
\hline 204 & A0A087WZT3 & A0A087WZT3 & BolA-like protein 2 & 2.62918 & 3.9663 \\
\hline 205 & LANC1 & O43813 & $\begin{array}{l}\text { Glutathione S-transferase } \\
\text { LANCL1 }\end{array}$ & 4.28469 & 3.09074 \\
\hline 206 & CND3 & Q9BPX3 & Condensin complex subunit 3 & 2.9969 & 2.66232 \\
\hline 207 & AIMP2 & Q13155 & $\begin{array}{l}\text { Aminoacyl tRNA synthase } \\
\text { complex-interacting multifunc- } \\
\text { tional protein } 2\end{array}$ & 3.83739 & 3.0309 \\
\hline 208 & G3V325 & G3V325 & ATP5MF-PTCD1 readthrough & 4.35305 & 4.77429 \\
\hline 209 & TGM2 & P21980 & $\begin{array}{l}\text { Protein-glutamine gamma- } \\
\text { glutamyltransferase } 2\end{array}$ & 3.47902 & 2.55024 \\
\hline 210 & RS18 & P62269 & 40 S ribosomal protein $\mathrm{S} 18$ & 3.20805 & 4.17992 \\
\hline 211 & RS14 & P62263 & $40 S$ ribosomal protein $\mathrm{S} 14$ & 2.67833 & 4.25944 \\
\hline 212 & C9JXB8 & C9JXB8 & $60 S$ ribosomal protein L24 & 2.98512 & 4.69503 \\
\hline 213 & MGST2 & Q99735 & $\begin{array}{l}\text { Microsomal glutathione S- } \\
\text { transferase } 2\end{array}$ & 2.68604 & 3.32233 \\
\hline 214 & RL13A & P40429 & $60 S$ ribosomal protein $\mathrm{L} 13 \mathrm{a}$ & 2.87139 & 4.09016 \\
\hline 215 & SNR40 & Q96DI7 & $\begin{array}{l}\text { U5 small nuclear ribonucleo- } \\
\text { protein } 40 \mathrm{kDa} \text { protein }\end{array}$ & 2.83902 & 2.94416 \\
\hline 216 & SRP54 & P61011 & $\begin{array}{l}\text { Signal recognition particle } 54 \\
\text { kDa protein }\end{array}$ & 3.37647 & 3.10435 \\
\hline 217 & CUL4A & Q13619 & Cullin-4A & 5.00644 & 2.58406 \\
\hline 218 & UCK2 & Q9BZX2 & Uridine-cytidine kinase 2 & 2.99612 & 2.78459 \\
\hline 219 & K7EP65 & K7EP65 & $60 S$ ribosomal protein L22 & 3.68703 & 6.73393 \\
\hline 220 & RS19 & P39019 & $40 S$ ribosomal protein S19 & 3.54415 & 7.35693 \\
\hline 221 & TSYL1 & Q9H0U9 & $\begin{array}{l}\text { Testis-specific Y-encoded-like } \\
\text { protein } 1\end{array}$ & 2.66813 & 3.09096 \\
\hline 222 & E9PJD9 & E9PJD9 & $60 S$ ribosomal protein L27a & 3.04224 & 5.20122 \\
\hline 223 & VPS45 & Q9NRW7 & $\begin{array}{l}\text { Vacuolar protein sorting- } \\
\text { associated protein } 45\end{array}$ & 2.73297 & 4.2448 \\
\hline 224 & M0R3D6 & M0R3D6 & $60 S$ ribosomal protein $\mathrm{L} 18 \mathrm{a}$ & 3.29172 & 4.15871 \\
\hline 225 & RS6 & P62753 & $40 S$ ribosomal protein S6 & 2.85538 & 3.91982 \\
\hline 226 & H7C2W9 & H7C2W9 & $60 S$ ribosomal protein L31 & 2.97225 & 6.33005 \\
\hline 227 & NUCL & P19338 & Nucleolin & 3.49248 & 4.59942 \\
\hline 228 & A0A0D9SG12 & A0A0D9SG12 & $\begin{array}{l}\text { ATP-dependent RNA helicase } \\
\text { DDX3X }\end{array}$ & 2.69323 & 4.56215 \\
\hline 229 & CDC73 & Q6P1J9 & Parafibromin & 3.60285 & 2.99202 \\
\hline 230 & APT & P07741 & $\begin{array}{l}\text { Adenine } \\
\text { phosphoribosyltransferase }\end{array}$ & 3.34193 & 2.61598 \\
\hline 31 & E7ENU7 & E7ENU7 & Ribosomal protein L15 & 3.97322 & 2.8185 \\
\hline 232 & G3BP1 & Q13283 & Ras GTPase-activating & 3.04396 & 3.61278 \\
\hline
\end{tabular}




\begin{tabular}{|c|c|c|c|c|c|}
\hline & & & protein-binding protein 1 & & \\
\hline 233 & K7ERT8 & K7ERT8 & $60 S$ ribosomal protein L23a & 4.22784 & 5.36401 \\
\hline 234 & J3QQ67 & J3QQ67 & $60 S$ ribosomal protein L18 & 2.66443 & 2.95231 \\
\hline 235 & XRCC6 & P12956 & $\begin{array}{l}\text { X-ray repair cross- } \\
\text { complementing protein } 6\end{array}$ & 3.48879 & 3.75823 \\
\hline 236 & MOROR2 & MOR0R2 & 40 S ribosomal protein S5 & 4.26617 & 5.14718 \\
\hline 237 & RFC5 & P40937 & Replication factor $\mathrm{C}$ subunit 5 & 4.36703 & 3.35394 \\
\hline 238 & F8W727 & F8W727 & 60 S ribosomal protein L32 & 3.74762 & 5.39745 \\
\hline 239 & MD2L1 & Q13257 & $\begin{array}{l}\text { Mitotic spindle assembly } \\
\text { checkpoint protein MAD2A }\end{array}$ & 2.92545 & 2.98028 \\
\hline 240 & D6RAN4 & D6RAN4 & 60 S ribosomal protein L9 & 3.31616 & 4.19725 \\
\hline 241 & RL4 & P36578 & $60 S$ ribosomal protein L4 & 2.85467 & 3.7287 \\
\hline 242 & RL36 & Q9Y3U8 & $60 S$ ribosomal protein L36 & 4.53022 & 5.5977 \\
\hline 243 & SSBP & Q04837 & $\begin{array}{l}\text { Single-stranded DNA-binding } \\
\text { protein, mitochondrial }\end{array}$ & 5.65442 & 4.22935 \\
\hline 244 & RS10 & P46783 & 40 S ribosomal protein $\mathrm{S} 10$ & 3.25188 & 3.02068 \\
\hline 245 & C9JZI1 & C9JZI1 & Replication factor $C$ subunit 4 & 2.62627 & 3.47232 \\
\hline 246 & XRCC5 & P13010 & $\begin{array}{l}\text { X-ray repair cross- } \\
\text { complementing protein } 5\end{array}$ & 2.60763 & 3.48406 \\
\hline 247 & AMPN & P15144 & Aminopeptidase $\mathrm{N}$ & 3.75088 & 3.23543 \\
\hline 248 & GTF2I & P78347 & General transcription factor II-I & 2.60151 & 3.52247 \\
\hline 249 & RS7 & P62081 & 40 S ribosomal protein $S 7$ & 3.97012 & 3.42622 \\
\hline 250 & C9JQV0 & C9JQV0 & $\begin{array}{l}\text { Uncharacterized protein } \\
\text { C7orf50 }\end{array}$ & 2.86837 & 2.93894 \\
\hline 251 & ACOX3 & O15254 & $\begin{array}{l}\text { Peroxisomal acyl-coenzyme A } \\
\text { oxidase } 3\end{array}$ & 2.7579 & 4.58583 \\
\hline
\end{tabular}

Table 18: Top 5 networks identified from IPA analysis in WT-tau expressing cells

\begin{tabular}{clc}
\hline ID & \multicolumn{1}{c}{ Associated network functions } & Score \\
\hline 1 & RNA damage and repair, protein synthesis, cancer & 61 \\
2 & $\begin{array}{l}\text { DNA replication, recombination, and repair, cellular assembly and } \\
\text { organization, cell morphology }\end{array}$ & 41 \\
3 & Cell cycle, cellular function and maintenance, molecular transport & 28 \\
4 & $\begin{array}{l}\text { Cellular movement, haematological system development and func- } \\
\text { tion, immune cell trafficking }\end{array}$ & 21 \\
5 & $\begin{array}{l}\text { Organismal injury and abnormalities, cellular movement, skeletal } \\
\text { and muscular system development and function }\end{array}$ & 17 \\
\hline
\end{tabular}


Table 19: Top 5 networks identified from IPA analysis in P301L-tau expressing cells

ID Associated network functions Score

1 RNA damage and repair, protein synthesis, cancer 55

2 Cellular assembly and organization, cellular compromise, cellular 52 function and maintenance

3 Nucleic acid metabolism, small molecule biochemistry, digestive 27 system development and function

4 Cellular compromise, developmental disorder, endocrine system 22 disorders

5 Developmental disorder, endocrine system disorders, organ morphology

2

7




\section{Bibliography}

Abu-Rumeileh S, Capellari S, Parchi P. Rapidly progressive Alzheimer's disease: contributions to clinical-pathological definition and diagnosis. J Alzheimers Dis. 2018; 63(3): 887-897.

Abu-Rumeileh S, Lattanzio F, Stanzani Maserati M, Rizzi R, Capellari S, Parchi P. Diagnostic accuracy of a combined analysis of cerebrospinal fluid t-PrP, t-tau, p-tau, and A $\beta 42$ in the differential diagnosis of Creutzfeldt-Jakob disease from Alzheimer's disease with emphasis on atypical disease variants. $J$ Alzheimers Dis. 2017; 55(4): 1471-1480.

Al-Asmary SM, Kadasah S, Arfin M, Tariq M, Al-Asmari A. Apolipoprotein E polymorphism is associated with susceptibility to schizophrenia among Saudis. Arch Med Sci. 2015; 11(4): 869-876.

Alberti S, Halfmann R, King O, Kapila A, Lindquist S. A systematic survey identifies prions and illuminates sequence features of prionogenic proteins. Cell. 2009; 137(1): 146-158.

Alzheimer's Association. Alzheimer's disease facts and figures. Alzheimers Dement. 2019; 15(3): 321-387.

Anderson P, Kedersha N, Ivanov P. Stress granules, P-bodies and cancer. Biochim Biophys Acta. 2015; 1849(7): 861-870.

Anderson P, Kedersha N. RNA granules. J Cell Biol. 2006; 172(6): 803-808.

Anderson P, Kedersha N. RNA granules: post-transcriptional and epigenetic modulators of gene expression. Nat Rev Mol Cell Biol. 2009; 10(6): 430-436.

Andreyev AY, Kushnareva YE, Starkov AA. Mitochondrial metabolism of reactive oxygen species. Biochemistry (Mosc). 2005; 70(2): 200-214.

Anji A, Kumari M. Guardian of genetic messenger-RNA-binding proteins. Biomolecules. 2016; 6(1): 4. 
Apicco DJ, Ash PEA, Maziuk B, LeBlang C, Medalla M, Al Abdullatif A, Ferragud A, Botelho E, Ballance HI, Dhawan U, Boudeau S, Cruz AL, Kashy D, Wong A, Goldberg LR, Yazdani N, Zhang C, Ung CY, Tripodis Y, Kanaan NM, Ikezu T, Cottone P, Leszyk J, Li H, Luebke J, Bryant CD, Wolozin B. Reducing the RNA binding protein TIA1 protects against tau-mediated neurodegeneration in vivo. Nat Neurosci. 2018; 21(1): 72-80.

Aulas A, Vande Velde C. Alterations in stress granule dynamics driven by TDP-43 and FUS: a link to pathological inclusions in ALS? Front Cell Neurosci. 2015; 9: 423.

Ba M, Li X, Ng KP, Pascoal TA, Mathotaarachchi S, Rosa-Neto P, Gauthier S. The prevalence and biomarkers' characteristic of rapidly progressive Alzheimer's disease from the Alzheimer's disease neuroimaging initiative database. Alzheimers Dement (N Y). 2017; 3(1): 107-113.

Bai B, Hales CM, Chen PC, Gozal Y, Dammer EB, Fritz JJ, Wang X, Xia Q, Duong DM, Street C, Cantero G, Cheng D, Jones DR, Wu Z, Li Y, Diner I, Heilman CJ, Rees HD, Wu H, Lin L, Szulwach KE, Gearing M, Mufson EJ, Bennett DA, Montine TJ, Seyfried NT, Wingo TS, Sun YE, Jin P, Hanfelt J, Willcock DM, Levey A, Lah JJ, Peng J. U1 small nuclear ribonucleoprotein complex and RNA splicing alterations in Alzheimer's disease. Proc Natl Acad Sci USA. 2013; 110(41): 16562-16567.

Balcerak A, Trebinska-Stryjewska A, Konopinski R, Wakula M, Grzybowska EA. RNA-protein interactions: disorder, moonlighting and junk contribute to eukaryotic complexity. Open Biol. 2019; 9(6): 190096.

Bali J, Gheinani AH, Zurbriggen S, Rajendran L. Role of genes linked to sporadic Alzheimer's disease risk in the production of A $\beta$-amyloid peptides. Proc Natl Acad Sci USA. 2012; 109(38): 15307-15311.

Baltz AG, Munschauer M, Schwanhausser B, Vasile A, Murakawa Y, Schueler M, Youngs N, Penfold-Brown D, Drew K, Milek M, Wyler E, Bonneau R, Selbach $\mathrm{M}$, Dieterich $\mathrm{C}$, Landthaler M. The mRNA-bound proteome and its global 
occupancy profile on protein-coding transcripts. Mol Cell. 2012; 46(5): 674690.

Banerjee S, Barraud P. Functions of double-stranded RNA-binding domains in nucleocytoplasmic transport. RNA Biol. 2014; 11(10): 1226-1232.

Barmada SJ, Skibinski G, Korb E, Rao EJ, Wu JY, Finkbeiner S. Cytoplasmic mislocalization of TDP-43 is toxic to neurons and enhanced by a mutation associated with familial amyotrophic lateral sclerosis. J Neurosci. 2010; 30(2): 639-649.

Batlle C, Iglesias V, Navarro S, Ventura S. Prion-like proteins and their computational identification in proteomes. Expert Rev Proteomics. 2017; 14(4): 335-350.

Beckmann BM, Horos R, Fischer B, Castello A, Eichelbaum K, Alleaume AM, Schwarzl T, Curk T, Foehr S, Huber W, Krijgsveld J, Hentze MW. The RNAbinding proteomes from yeast to man harbour conserved enigmRBPs. Nat Commun. 2015; 6: 10127.

Belfiore R, Rodin A, Ferreira E, Velazquez R, Branca C, Caccamo A, Oddo S. Temporal and regional progression of Alzheimer's disease-like pathology in 3xTg-AD mice. Aging Cell. 2019; 18(1): e12873.

Bertram L, Lill CM, Tanzi RE. The genetics of Alzheimer disease: back to the future. Neuron. 2010; 68(2): 270-281.

Bishof I, Dammer EB, Duong DM, Kundinger S, Gearing M, Lah JJ, Levey AI, Seyfried NT. RNA-binding proteins with basic acidic dipeptide (BAD) domains self-assemble and aggregate in Alzheimer's disease. J Biol Chem. 2018; 293(28): 11047-11066.

Bishop MT, Will RG, Manson JC. Defining sporadic Creutzfeldt-Jakob disease strains and their transmission properties. Proc Natl Acad Sci USA. 2010; 107(26): 12005-12010. 
Blennow K, de Leon MJ, Zetterberg H. Alzheimer's disease. Lancet. 2006; 368(9533): 387-403.

Boeynaems S, Alberti S, Fawzi NL, Mittag T, Polymenidou M, Rousseau F, Schymkowitz J, Shorter J, Wolozin B, Van Den Bosch L, Tompa P, Fuxreiter M. Protein phase separation: a new phase in cell biology. Trends Cell Biol. 2018; 28(6): 420-435.

Bolte S, Cordelieres FP. A guided tour into subcellular colocalization analysis in light microscopy. J Microsc. 2006; 224(Pt 3): 213-232.

Bosco DA, Lemay N, Ko HK, Zhou H, Burke C, Kwiatkowski TJ Jr, Sapp P, McKenna-Yasek D, Brown RH Jr, Hayward LJ. Mutant FUS proteins that cause amyotrophic lateral sclerosis incorporate into stress granules. Hum Mol Genet. 2010; 19(21): 4160-4175.

Braak H, Alafuzoff I, Arzberger T, Kretzschmar H, Del Tredici K. Staging of Alzheimer disease-associated neurofibrillary pathology using paraffin sections and immunocytochemistry. Acta Neuropathol. 2006; 112(4): 389-404.

Braak H, Braak E. Neuropathological stageing of Alzheimer-related changes. Acta Neuropathol. 1991; 82(4): 239-259.

Brady RM, Zinkowski RP, Binder LI. Presence of tau in isolated nuclei from human brain. Neurobiol Aging. 1995; 16(3): 479-486.

Brangwynne CP. Phase transitions and size scaling of membrane-less organelles. $J$ Cell Biol. 2013; 203(6): 875.

Brown RS. Zinc finger proteins: getting a grip on RNA. Curr Opin Struct Biol. 2005; 15(1): 94-98.

Brunello CA, Yan X, Huttunen HJ. Internalized tau sensitizes cells to stress by promoting formation and stability of stress granules. Sci Rep. 2016; 6: 30498.

Buchan JR, Kolaitis RM, Taylor JP, Parker R. Eukaryotic stress granules are cleared by autophagy and Cdc48/VCP function. Cell. 2013; 153(7): 1461-1474. 
Bucholtz N, Demuth I. DNA-repair in mild cognitive impairment and Alzheimer's disease. DNA Repair (Amst). 2013; 12(10): 811-816.

Bukar MM, Al-Hilaly YK, Serpell LC. Nuclear tau and its potential role in Alzheimer's disease. Biomolecules. 2016; 6(1): 9.

Calabretta S, Richard Sp. Emerging roles of disordered sequences in RNA-binding proteins. Trends Biochem Sci. 2015; 40(11): 662-672.

Cao J, Hou J, Ping J, Cai D. Advances in developing novel therapeutic strategies for Alzheimer's disease. Mol Neurodegener. 2018; 13(1): 64.

Castello A, Fischer B, Eichelbaum K, Horos R, Beckmann BM, Strein C, Davey NE, Humphreys DT, Preiss T, Steinmetz LM, Krijgsveld J, Hentze MW. Insights into RNA biology from an atlas of mammalian mRNA-binding proteins. Cell. 2012; 149(6): 1393-1406.

Castello A, Fischer B, Frese CK, Horos R, Alleaume AM, Foehr S, Curk T, Krijgsveld $\mathrm{J}$, Hentze MW. Comprehensive identification of RNA-binding domains in human cells. Mol. Cell. 2016; 63(4): 696-710.

Castello A, Fischer B, Hentze MW, Preiss T. RNA-binding proteins in Mendelian disease. Trends Genet. 2013; 29(5): 318-327.

Castello A, Hentze MW, Preiss T. Metabolic enzymes enjoying new partnerships as RNA-binding proteins. Trends Endocrinol Metab. 2015; 26(12): 746-757.

Chen L, Liu B. Relationships between stress granules, oxidative stress, and neurodegenerative diseases. Oxid Med Cell Longev. 2017; 2017: 1809592.

Chen X, Guo C, Kong J. Oxidative stress in neurodegenerative diseases. Neural Regen Res. 2012; 7(5): 376-385.

Chitravas N, Jung RS, Kofskey DM, Blevins JE, Gambetti P, Leigh RJ, Cohen ML. Treatable neurological disorders misdiagnosed as Creutzfeldt-Jakob disease. Ann Neurol. 2011; 70(3): 437-444. 
Clery A, Blatter M, Allain FH. RNA recognition motifs: boring? Not quite. Curr Opin Struct Biol. 2008; 18(3): 290-298.

Cobley JN, Fiorello ML, Bailey DM. 13 reasons why the brain is susceptible to oxidative stress. Redox Biol. 2018; 15: 490-503.

Cohen ML, Kim C, Haldiman T, ElHag M, Mehndiratta P, Pichet T, Lissemore F, Shea M, Cohen Y, Chen W, Blevins J, Appleby BS, Surewicz K, Surewicz WK, Sajatovic M, Tatsuoka C, Zhang S, Mayo P, Butkiewicz M, Haines JL, Lerner AJ, Safar JG. Rapidly progressive Alzheimer's disease features distinct structures of amyloid-beta. Brain. 2015; 138(Pt 4): 1009-1022.

Collinge J. Prion diseases of humans and animals: their causes and molecular basis. Annu Rev Neurosci. 2001; 24: 519-550.

Conlon EG, Manley JL. RNA-binding proteins in neurodegeneration: mechanisms in aggregate. Genes Dev. 2017; 31(15): 1509-1528.

Cookson MR. RNA-binding proteins implicated in neurodegenerative diseases. Wiley Interdiscip Rev RNA. 2017; 8(1).

Coppin L, Leclerc J, Vincent A, Porchet N, Pigny P. Messenger RNA life-cycle in cancer cells: emerging role of conventional and non-conventional RNAbinding proteins? Int J Mol Sci. 2018; 19(3). E650.

Couthouis J, Hart MP, Shorter J, DeJesus-Hernandez M, Erion R, Oristano R, Liu AX, Ramos D, Jethava N, Hosangadi D, Epstein J, Chiang A, Diaz Z, Nakaya T, Ibrahim F, Kim HJ, Solski JA, Williams KL, Mojsilovic-Petrovic J, Ingre C, Boylan K, Graff-Radford NR, Dickson DW, Clay-Falcone D, Elman L, McCluskey L, Greene R, Kalb RG, Lee VM, Trojanowski JQ, Ludolph A, Robberecht W, Andersen PM, Nicholson GA, Blair IP, King OD, Bonini NM, Van D, V, Rademakers R, Mourelatos Z, Gitler AD. A yeast functional screen predicts new candidate ALS disease genes. Proc Natl Acad Sci USA. 2011; 108(52): 20881-20890. 
Crous-Bou M, Minguillón C, Gramunt N, Molinuevo JL. Alzheimer's disease prevention: from risk factors to early intervention. Alzheimers Res Ther. 2017; 9(1): 71.

Cushman M, Johnson BS, King OD, Gitler AD, Shorter J. Prion-like disorders: blurring the divide between transmissibility and infectivity. J Cell Sci. 2010; 123(Pt 8): 1191-1201.

Dassi E, Malossini A, Re A, Mazza T, Tebaldi T, Caputi L, Quattrone A. AURA: Atlas of UTR Regulatory Activity. Bioinformatics. 2012; 28(1): 142-144.

Delobel P, Mailliot C, Hamdane M, Sambo AV, Begard S, Violleau A, Delacourte A, Buee L. Stable-tau overexpression in human neuroblastoma cells: an open door for explaining neuronal death in tauopathies. Ann N Y Acad Sci. 2003; 1010: 623-634.

Diner I, Hales CM, Bishof I, Rabenold L, Duong DM, Yi H, Laur O, Gearing M, Troncoso J, Thambisetty M, Lah JJ, Levey Al, Seyfried NT. Aggregation properties of the small nuclear ribonucleoprotein U1-70K in Alzheimer disease. J Biol Chem. 2014; 289(51): 35296-35313.

Ding Q, Markesbery WR, Chen Q, Li F, Keller JN. Ribosome dysfunction is an early event in Alzheimer's disease. J Neurosci. 2005; 25(40): 9171-9175.

Ding $Y$, Tang $Y$, Kwok CK, Zhang Y, Bevilacqua PC, Assmann SM. In vivo genomewide profiling of RNA secondary structure reveals novel regulatory features. Nature. 2014; 505(7485): 696-700.

Doody RS, Thomas RG, Farlow M, Iwatsubo T, Vellas B, Joffe S, Kieburtz K, Raman R, Sun X, Aisen PS, Siemers E, Liu-Seifert H, Mohs R. Phase 3 trials of solanezumab for mild-to-moderate Alzheimer's disease. N Engl J Med. 2014; 370(4): 311-21.

Drummond E, Nayak S, Faustin A, Pires G, Hickman RA, Askenazi M, Cohen M, Haldiman T, Kim C, Han X, Shao Y, Safar JG, Ueberheide B, Wisniewski T. Proteomic differences in amyloid plaques in rapidly progressive and sporadic Alzheimer's disease. Acta Neuropathol. 2017; 133(6): 933-954. 
Duss O, Michel E, Diarra dit KN, Schubert M, Allain FH. Molecular basis for the wide range of affinity found in Csr/Rsm protein-RNA recognition. Nucleic Acids Res. 2014; 42(8): 5332-5346.

Dyson HJ, Wright PE. Intrinsically unstructured proteins and their functions. Nat Rev Mol Cell Biol. 2005; 6(3): 197-208.

Edwards lii GA, Gamez N, Escobedo G Jr, Calderon O, Moreno-Gonzalez I. Modifiable risk factors for Alzheimer's disease. Front Aging Neurosci. 2019; 11: 146 .

Evans HT, Benetatos J, van RM, Bodea LG, Gotz J. Decreased synthesis of ribosomal proteins in tauopathy revealed by non-canonical amino acid labelling. EMBO J. 2019; 38(13): e101174.

Everts B, Amiel E, Huang SC, Smith AM, Chang CH, Lam WY, Redmann V, Freitas TC, Blagih J, van der Windt GJ, Artyomov MN, Jones RG, Pearce EL, Pearce EJ. TLR-driven early glycolytic reprogramming via the kinases TBK1-IKKvarepsilon supports the anabolic demands of dendritic cell activation. Nat Immunol. 2014; 15(4): 323-332.

Feng Q, Cheng B, Yang R, Sun FY, Zhu CQ. Dynamic changes of phosphorylated tau in mouse hippocampus after cold water stress. Neurosci Lett. 2005; 388(1): 13-16.

Feng Y, Xia Y, Yu G, Shu X, Ge H, Zeng K, Wang J, Wang X. Cleavage of GSK3beta by calpain counteracts the inhibitory effect of Ser9 phosphorylation on GSK-3beta activity induced by $\mathrm{H}_{2} \mathrm{O}_{2}$. J Neurochem. 2013; 126(2): 234-242.

Fernández-Nogales $M$, Santos-Galindo $M$, Merchán-Rubira J, Hoozemans JJM, Rábano A, Ferrer I, Avila J, Hernández F, Lucas JJ. Tau-positive nuclear indentations in P301S tauopathy mice. Brain Pathol. 2017 27(3): 314-322.

Ferri CP, Prince M, Brayne C, Brodaty H, Fratiglioni L, Ganguli M, Hall K, Hasegawa K, Hendrie H, Huang $\mathrm{Y}$, Jorm A, Mathers C, Menezes PR, Rimmer E, Scazufca M. Global prevalence of dementia: a Delphi consensus study. Lancet. 2005; 366(9503): 2112-2117. 
Fillenbaum GG, van Belle G, Morris JC, Mohs RC, Mirra SS, Davis PC, Tariot PN, Silverman JM, Clark CM, Welsh-Bohmer KA, Heyman A. Consortium to Establish a Registry for Alzheimer's Disease (CERAD): the first twenty years. Alzheimers Dement. 2008; 4(2): 96-109.

Förstl H, Kurz A. Clinical features of Alzheimer's disease. Eur Arch Psychiatry Clin Neurosci. 1999; 249(6): 288-290.

Frost B, Hemberg M, Lewis J, Feany MB. Tau promotes neurodegeneration through global chromatin relaxation. Nat Neurosci. 2014; 17(3): 357-366.

Gacia M, Safranow K, Styczyńska M, Jakubowska K, Pepłońska B, ChodakowskaZebrowska M, Przekop I, Słowik A, Golańska E, Hułas-Bigoszewska K, Chlubek D, Religa D, Zekanowski C, Barcikowska M. Prion protein gene M129 allele is a risk factor for Alzheimer's disease. J Neural Transm (Vienna). 2006;113(11):1747-51.

Galietta A, Gunby RH, Redaelli S, Stano P, Carniti C, Bachi A, Tucker PW, Tartari CJ, Huang CJ, Colombo E, Pulford K, Puttini M, Piazza RG, Ruchatz H, Villa A, Donella-Deana A, Marin O, Perrotti D, Gambacorti-Passerini C. NPM/ALK binds and phosphorylates the RNA/DNA-binding protein PSF in anaplastic large-cell lymphoma. Blood. 2007; 110(7): 2600-2609.

Gao M, Arkov AL. Next generation organelles: structure and role of germ granules in the germline. Mol Reprod Dev. 2013; 80(8): 610-623.

GBD 2016 dementia collaborators. Global, regional, and national burden of Alzheimer's disease and other dementias, 1990-2016: a systematic analysis for the global burden of disease study 2016. Lancet Neurol. 2019 ;18(1): 88106.

Gerstberger S, Hafner M, Tuschl T. A census of human RNA-binding proteins. Nat Rev Genet. 2014; 15(12): 829-845.

Glisovic T, Bachorik JL, Yong J, Dreyfuss G. RNA-binding proteins and posttranscriptional gene regulation. FEBS Lett. 2008; 582(14): 1977-1986. 
Goate A, Hardy J. Twenty years of Alzheimer's disease-causing mutations. J Neurochem. 2012; 120(s1): 3-8.

Goedert M, Spillantini MG. Tau mutations in frontotemporal dementia FTDP-17 and their relevance for Alzheimer's disease. Biochim Biophys Acta. 2000; 1502(1): 110-21.

Goggin K, Beaudoin S, Grenier C, Brown AeA, Roucou X. Prion protein aggresomes are poly $(A)+$ ribonucleoprotein complexes that induce a PKR-mediated deficient cell stress response. Biochim Biophys Acta. 2008; 1783(3): 479491.

Gosai SJ, Foley SW, Wang D, Silverman IM, Selamoglu N, Nelson AD, Beilstein MA, Daldal F, Deal RB, Gregory BD. Global analysis of the RNA-protein interaction and RNA secondary structure landscapes of the Arabidopsis nucleus. Mol Cell. 2015; 57(2): 376-388.

Greco CM, Berman RF, Martin RM, Tassone F, Schwartz PH, Chang A, Trapp BD, Iwahashi C, Brunberg J, Grigsby J, HessI D, Becker EJ, Papazian J, Leehey MA, Hagerman RJ, Hagerman PJ. Neuropathology of fragile X-associated tremor/ataxia syndrome (FXTAS). Brain. 2006; 129(1): 243-255.

Grundke-lqbal I, Iqbal K, Quinlan M, Tung YC, Zaidi MS, Wisniewski HM. Microtubule-associated protein tau: a component of Alzheimer paired helical filaments. J. Biol. Chem. 1986; 261(13): 6084-6089.

Guerrero-Muñoz MJ, Gerson J, Castillo-Carranza DL. Tau oligomers: the toxic player at synapses in Alzheimer's disease. Front Cell Neurosci. 2015; 9: 464.

Guo L, Shorter J. It's raining liquids: RNA tunes viscoelasticity and dynamics of membraneless organelles. Mol Cell. 2015; 60(2): 189-192.

Hardy JA, Higgins GA. Alzheimer's disease: the amyloid cascade hypothesis . Science. 1992; 256(5054): 184-185.

Harrison AF, Shorter J. RNA-binding proteins with prion-like domains in health and disease. Biochem J. 2017; 474(8): 1417-1438. 
Helder S, Blythe AJ, Bond CS, Mackay JP. Determinants of affinity and specificity in RNA-binding proteins. Curr Opin Struct Biol. 2016; 38: 83-91.

Hennig S, Kong G, Mannen T, Sadowska A, Kobelke S, Blythe A, Knott GJ, lyer KS, Ho D, Newcombe EA, Hosoki K, Goshima N, Kawaguchi T, Hatters D, Trinkle-Mulcahy L, Hirose T, Bond CS, Fox AH. Prion-like domains in RNA binding proteins are essential for building subnuclear paraspeckles. $J$ Cell Biol. 2015; 210(4): 529-539.

Hentze MW, Castello A, Schwarzl T, Preiss T. A brave new world of RNA-binding proteins. Nat Rev Mol Cell Biol. 2018; 19(5): 327.

Herbert J, Lucassen PJ. Depression as a risk factor for Alzheimer's disease: Genes, steroids, cytokines and neurogenesis - What do we need to know? Front Neuroendocrinol. 2016; 41: 153-171.

Hernandez-Ortega K, Garcia-Esparcia P, Gil L, Lucas JJ, Ferrer I. Altered machinery of protein synthesis in Alzheimer's: from the nucleolus to the ribosome. Brain Pathol. 2016; 26(5): 593-605.

Heyd F, Lynch KW. Phosphorylation-dependent regulation of PSF by GSK3 controls CD45 alternative splicing. Mol Cell. 2010; 40(1): 126-137.

Hiller A, Quinn J, Schmidt P. Does psychological stress affect the progression of Parkinson's disease (N5.002). Neurology. 2017; 88(16 Supplement): N5.

Hoeijmakers L, Ruigrok SR, Amelianchik A, Ivan D, van Dam AM, Lucassen PJ, Korosi A. Early-life stress lastingly alters the neuroinflammatory response to amyloid pathology in an Alzheimer's disease mouse model. Brain Behav Immun. 2017; 63: 160-175.

Holt CE, Bullock SL. Subcellular mRNA localization in animal cells and why it matters. Science. 2009; 326(5957): 1212-1216.

Hoover BR, Reed MN, Su J, Penrod RD, Kotilinek LA, Grant MK, Pitstick R, Carlson GA, Lanier LM, Yuan LL, Ashe KH, Liao D. Tau mislocalization to dendritic 
spines mediates synaptic dysfunction independently of neurodegeneration. Neuron. 2010; 68(6): 1067-81.

Huang Q, Yang L, Luo J, Guo L, Wang Z, Yang X, Jin W, Fang Y, Ye J, Shan B, Zhang Y. SWATH enables precise label-free quantification on proteome scale. Proteomics. 2015; 15: 1215-1223.

Huang WJ, Zhang X, Chen WW. Role of oxidative stress in Alzheimer's disease. Biomed Rep. 2016; 15(5): 519-522.

Hyman AA, Weber CA, Jülicher F. Liquid-liquid phase separation in biology. Annu Rev Cell Dev Biol. 2014; 30(1): 39-58.

Ibáñez-Salazar A, Bañuelos-Hernández B, Rodríguez-Leyva I, Chi-Ahumada E, Monreal-Escalante E, Jiménez-Capdeville ME, Rosales-Mendoza S. Oxidative stress modifies the levels and phosphorylation state of tau protein in human fibroblasts. Front Neurosci. 2017; 11: 495.

Ishigaki S, Fujioka Y, Okada Y, Riku Y, Udagawa T, Honda D, Yokoi S, Endo K, Ikenaka K, Takagi S, Iguchi Y, Sahara N, Takashima A, Okano H, Yoshida M, Warita H, Aoki M, Watanabe H, Okado H, Katsuno M, Sobue. Altered tau isoform ratio caused by loss of FUS and SFPQ function leads to FTLD-like phenotypes. Cell Rep. 2017; 18(5): 1118-1131.

Islam $\mathrm{Bu}$, Jabir NR, Tabrez $\mathrm{S}$. The role of mitochondrial defects and oxidative stress in Alzheimer's disease. J Drug Target. 2019; 27(9): 932-942.

Iwahashi CK, Yasui DH, An HJ, Greco CM, Tassone F, Nannen K, Babineau B, Lebrilla CB, Hagerman RJ, Hagerman PJ. Protein composition of the intranuclear inclusions of FXTAS. Brain. 2006; 129(1): 256-271.

Jaafar L, Li Z, Li S, Dynan WS. SFPQ•NONO and XLF function separately and together to promote DNA double-strand break repair via canonical nonhomologous end joining. Nucleic Acids Res. 2017; 45(4): 1848-1859.

Jain S, Parker R. The discovery and analysis of P bodies. Adv Exp Med Biol. 2013; 768: 23-43. 
Jain S, Wheeler JR, Walters RW, Agrawal A, Barsic A, Parker R. ATPase-modulated stress granules contain a diverse proteome and substructure. Cell. 2016; 164(3): 487-498.

Janga SC, Mittal N. Construction, structure and dynamics of post-transcriptional regulatory network directed by RNA-binding proteins. Adv Exp Med Biol. 2011; 722: 103-117.

Jansen C, Parchi P, Capellari S, Ibrahim-Verbaas CA, Schuur M, Strammiello R, Corrado P, Bishop MT, van Gool WA, Verbeek MM, Baas F, van SW, Spliet WG, Jansen GH, van Duijn CM, Rozemuller AJ. Human prion diseases in the Netherlands (1998-2009): clinical, genetic and molecular aspects. PLoS One. 2012; 7(4): e36333.

Johnson RT, Gibbs CJ, Jr. Creutzfeldt-Jakob disease and related transmissible spongiform encephalopathies. N Engl J Med. 1998; 339(27): 1994-2004.

Justice NJ. The relationship between stress and Alzheimer's disease. Neurobiol Stress. 2018; 8: 127-133.

Kalaria RN, Maestre GE, Arizaga R, Friedland RP, Galasko D, Hall K, Luchsinger JA, Ogunniyi A, Perry EK, Potocnik F, Prince M, Stewart R, Wimo A, Zhang ZX, Antuono P. Alzheimer's disease and vascular dementia in developing countries: prevalence, management, and risk factors. Lancet Neurol. 2008; 7(9): 812-826.

Kanai Y, Dohmae N, Hirokawa N. Kinesin transports RNA: isolation and characterization of an RNA-transporting granule. Neuron. 2004; 43(4): 513525.

Karch CM, Goate AM. Alzheimer's disease risk genes and mechanisms of disease pathogenesis. Biol Psychiatry. 2015; 77(1): 43-51.

Kato M, Han TW, Xie, Shi K, Du X, Wu LC, Mirzaei H, Goldsmith EJ, Longgood J, Pei J, Grishin NV, Frantz DE, Schneider JW, Chen S, Li L, Sawaya MR, Eisenberg D, Tycko R, McKnight SL. Cell-free formation of RNA granules: 
low complexity sequence domains form dynamic fibers within hydrogels. Cell. 2012; 149(4): 753-767.

Ke YD, Dramiga J, Schutz U, Kril JJ, Ittner LM, Schroder H, Gotz J. Tau-mediated nuclear depletion and cytoplasmic accumulation of SFPQ in Alzheimer's and Pick's disease. PLoS One. 2012; 7(4): e35678.

King OD, Gitler AD, Shorter J. The tip of the iceberg: RNA-binding proteins with prion-like domains in neurodegenerative disease. Brain Res. 2012; 1462: 61-80.

Knott GJ, Bond CS, Fox AH. The DBHS proteins SFPQ, NONO and PSPC1: a multipurpose molecular scaffold. Nucleic Acids Res. 2016; 44(9): 39894004.

Korneyev AY. Stress-induced tau phosphorylation in mouse strains with different brain Erk $1+2$ immunoreactivity. Neurochem Res. 1998; 23(12): 15391543.

Krasemann S, Madore C, Cialic R, Baufeld C, Calcagno N, El Fatimy R, Beckers L, O'Loughlin E, Xu Y, Fanek Z, Greco DJ, Smith ST, Tweet G, Humulock Z, Zrzavy $T$, Conde-Sanroman $P$, Gacias $M$, Weng $Z$, Chen $H$, Tjon E, Mazaheri F, Hartmann K, Madi A, Ulrich JD, Glatzel M, Worthmann A, Heeren J, Budnik B, Lemere C, Ikezu T, Heppner FL, Litvak V, Holtzman DM, Lassmann H, Weiner HL, Ochando J, Haass C, Butovsky O. The TREM2-APOE pathway drives the transcriptional phenotype of dysfunctional microglia in neurodegenerative diseases. Immunity. 2017; 47(3): 566-581.

Krbot K, Glatzel M. Rapidly progressive Alzheimer's disease and sporadic Creutzfeldt-Jakob disease: comparison of clinical and neuropathological features. Alzheimers Dement. 2018; 14(7): 738-739.

Kunde SA, Musante L, Grimme A, Fischer U, Muller E, Wanker EE, Kalscheuer VM. The X-chromosome-linked intellectual disability protein PQBP1 is a component of neuronal RNA granules and regulates the appearance of stress granules. Hum Mol Genet. 2011; 20824: 4916-4931. 
Lambert MP, Sabo S, Zhang C, Enam SA, Klein WL. Constitutive Alzheimer's-type tau epitopes in a neuritogenic rat CNS cell line. Neurobiol Aging. 1995; 16(4): 583-589.

Lancaster AK, Nutter-Upham A, Lindquist S, King OD. PLAAC: a web and command-line application to identify proteins with prion-like amino acid composition. Bioinformatics. 2014; 30(17): 2501-2502.

Langstrom NS, Anderson JP, Lindroos HG, Winblad B, Wallace WC. Alzheimer's disease-associated reduction of polysomal mRNA translation. Brain Res Mol Brain Res. 1989; 5(4): 259-269.

Lattanzio F, Abu-Rumeileh S, Franceschini A, Kai H, Amore G, Poggiolini I, Rossi M, Baiardi S, McGuire L, Ladogana A, Pocchiari M, Green A, Capellari S, Parchi P. Prion-specific and surrogate CSF biomarkers in Creutzfeldt-Jakob disease: diagnostic accuracy in relation to molecular subtypes and analysis of neuropathological correlates of $\mathrm{p}$-tau and Abeta42 levels. Acta Neuropathol. 2017; 133(4): 559-578.

Lesuis SL, Maurin H, Borghgraef P, Lucassen PJ, Van LF, Krugers HJ. Positive and negative early life experiences differentially modulate long term survival and amyloid protein levels in a mouse model of Alzheimer's disease. Oncotarget. 2016; 7(26): 39118-39135.

Li F, Zheng Q, Ryvkin P, Dragomir I, Desai Y, Aiyer S, Valladares O, Yang J, Bambina S, Sabin LR, Murray JI, Lamitina T, Raj A, Cherry S, Wang LS, Gregory BD. Global analysis of RNA secondary structure in two metazoans. Cell Rep. 2012; 1(1): 69-82.

Li L, Jiang Y, Hu W, Tung YC, Dai C, Chu D, Gong CX, Iqbal K, Liu F. Pathological alterations of tau in Alzheimer's disease and 3xTg-AD mouse brains. Mol Neurobiol. 2019; 56(9): 6168-6183.

Li L, Lindquist S. Creating a protein-based element of inheritance. Science. 2000; 287(5453): 661-664. 
Li Q, Lau A, Morris TJ, Guo L, Fordyce CB, Stanley EF. A syntaxin 1, Galpha(o), and $\mathrm{N}$-type calcium channel complex at a presynaptic nerve terminal: analysis by quantitative immunocolocalization. J Neurosci. 2004; 24(16): 4070-4081.

Li Q, Lee JA, Black DL. Neuronal regulation of alternative pre-mRNA splicing. Nat Rev Neurosci. 2007; 8(11): 819-831.

Li YR, King OD, Shorter J, Gitler AD. Stress granules as crucibles of ALS pathogenesis. J Cell Biol. 2013; 201(3): 361-372.

Liao Y, Wang J, Jaehnig EJ, Shi Z, Zhang B. WebGestalt 2019: gene set analysis toolkit with revamped Uls and APIs. Nucleic Acids Res. 2019; 47(W1): W199-W205.

Liu EY, Cali CP, Lee EB. RNA metabolism in neurodegenerative disease. Dis Models Mech. 2017; 10(5): 509.

Liu-Yesucevitz L, Bilgutay A, Zhang YJ, Vanderweyde T, Citro A, Mehta T, Zaarur N, McKee A, Bowser R, Sherman M, Petrucelli L, Wolozin B. Tar DNA binding protein-43 (TDP-43) associates with stress granules: analysis of cultured cells and pathological brain tissue. PLoS One. 2010; 5(10): e13250.

Liu-Yesucevitz L, Lin AY, Ebata A, Boon JY, Reid W, Xu YF, Kobrin K, Murphy GJ, Petrucelli L, Wolozin B. ALS-Linked mutations enlarge TDP-43-enriched neuronal RNA granules in the dendritic arbor. $J$ Neurosci. 2014; 34(12): 4167-4174.

Livak KJ, Schmittgen TD. Analysis of relative gene expression data using real-time quantitative PCR and the $2^{-\Delta \Delta C t}$ Method. Methods. 2001; 25(4): 402-408.

Llorens F, Schmitz M, Karch A, Cramm M, Lange P, Gherib K, Varges D, Schmidt C, Zerr I, Stoeck K. Comparative analysis of cerebrospinal fluid biomarkers in the differential diagnosis of neurodegenerative dementia. Alzheimers Dement. 2016; 12(5): 577-589.

Lopes S, Vaz-Silva Jo, Pinto V, Dalla C, Kokras N, Bedenk B, Mack N, Czisch M, Almeida OFX, Sousa N, Sotiropoulos I. Tau protein is essential for stress- 
induced brain pathology. Proc Natl Acad Sci USA. 2016; 113(26): E3755E3763.

Lovell MA, Xiong S, Xie C, Davies P, Markesbery WR. Induction of hyperphosphorylated tau in primary rat cortical neuron cultures mediated by oxidative stress and glycogen synthase kinase-3. J Alzheimers Dis. 2004; 6(6): 659-671.

Lovestone S, Boada M, Dubois B, Hüll M, Rinne JO, Huppertz HJ, Calero M, Andrés MV, Gómez-Carrillo B, León T, del Ser T. A phase II trial of tideglusib in Alzheimer's disease. J Alzheimers Dis. 2015; 45(1): 75-88.

Lowery LA, Rubin J, Sive H. whitesnake/sfpq is required for cell survival and neuronal development in the zebrafish. Dev Dyn. 2007; 236(5): 1347-1357.

Lu J, Li T, He R, Bartlett PF, Götz J. Visualizing the microtubule-associated protein tau in the nucleus. Sci China Life Sci. 2014; 57(4): 422-31.

Lu J, Miao J, Su T, Liu Y, He R. Formaldehyde induces hyperphosphorylation and polymerization of Tau protein both in vitro and in vivo. Biochim Biophys Acta. 2013; 1830(8): 4102-4116.

Lu J, Shu R, Zhu Y. Dysregulation and dislocation of SFPQ disturbed DNA organization in Alzheimer's disease and frontotemporal dementia. $J$ Alzheimers Dis. 2018; 61(4): 1311-1321.

Luisier R, Tyzack GE, Hall CE, Mitchell JS, Devine H, Taha DM, Malik B, Meyer I, Greensmith L, Newcombe J, Ule J, Luscombe NM, Patani R. Intron retention and nuclear loss of SFPQ are molecular hallmarks of ALS. Nature Communications. 2018; 9(1): 2010.

Lukong KE, Huot ME, Richard S. BRK phosphorylates PSF promoting its cytoplasmic localization and cell cycle arrest. Cell Signal. 2009; 21(9): 14151422.

Lunde BM, Moore C, Varani G. RNA-binding proteins: modular design for efficient function. Nat Rev Mol Cell Biol. 2007; 8(6): 479-490. 
Magalingam KB, Radhakrishnan A, Ping NS, Haleagrahara N. Current concepts of neurodegenerative mechanisms in Alzheimer's disease. Biomed Res Int. 2018; 2018: 3740461.

Mahley RW. Apolipoprotein E: from cardiovascular disease to neurodegenerative disorders. J Mol Med (Berl) 2016; 94(7): 739-746.

Maina MB, Bailey LJ, Doherty AJ, Serpell LC. The involvement of $A \beta 42$ and tau in nucleolar and protein synthesis machinery dysfunction. Front Cell Neurosci. 2018; 12: 220.

March ZM, King OD, Shorter J. Prion-like domains as epigenetic regulators, scaffolds for subcellular organization, and drivers of neurodegenerative disease. Brain Res. 2016; 1647: 9-18.

Marchese D, de Groot NS, Lorenzo GN, Livi CM, Tartaglia GG. Advances in the characterization of RNA-binding proteins. Wiley Interdiscip Rev RNA. 2016; 7(6): 793-810.

Martin KC, Ephrussi A. mRNA localization: gene expression in the spatial dimension. Cell. 2009; 136(4): 719-730.

Masison DC, Maddelein ML, Wickner RB. The prion model for [URE3] of yeast: Spontaneous generation and requirements for propagation. Proc Natl Acad Sci USA. 1997; 94(23): 12503-12508.

Mastroeni D, Grover A, Delvaux E, Whiteside C, Coleman PD, Rogers J. Epigenetic mechanisms in Alzheimer's disease. Neurobiol Aging. 2011; 32(7): 11611180.

Mays CE, Armijo E, Morales R, Kramm C, Flores A, Tiwari A, Bian J, Telling GC, Pandita TK, Hunt CR, Soto C. Prion disease is accelerated in mice lacking stress-induced heat shock protein 70 (HSP70). J Biol Chem. 2019; 294(37): 13619-13628.

Maziuk B, Ballance HI, Wolozin B. Dysregulation of RNA binding protein aggregation in neurodegenerative disorders. Front Mol Neurosci. 2017; 10: 89. 
Maziuk BF, Apicco DJ, Cruz AL, Jiang L, Ash PEA, da Rocha EL, Zhang C, Yu WH, Leszyk J, Abisambra JF, Li H, Wolozin B. RNA binding proteins co-localize with small tau inclusions in tauopathy. Acta Neuropathol Commun. 2018; 6(1): 71 .

McEwen E, Kedersha N, Song B, Scheuner D, Gilks N, Han A, Chen JJ, Anderson $P$, Kaufman RJ. Heme-regulated inhibitor kinase-mediated phosphorylation of eukaryotic translation initiation factor 2 inhibits translation, induces stress granule formation, and mediates survival upon arsenite exposure. $J$ Biol Chem. 2005; 280(17): 16925-16933.

McMahon AC, Rahman R, Jin H, Shen JL, Fieldsend A, Luo W, Rosbash M. TRIBE: hijacking an RNA-editing enzyme to identify cell-specific targets of RNAbinding proteins. Cell. 2016; 165(3): 742-753.

Mendez MF. Early-onset Alzheimer disease. Neurol Clin. 2017; 35(2): 263-281.

Meissner B, Kallenberg K, Sanchez-Juan P, Collie D, Summers DM, Almonti S, Collins SJ, Smith P, Cras P, Jansen GH, Brandel JP, Coulthart MB, Roberts H, Van EB, Galanaud D, Mellina V, Will RG, Zerr I. MRI lesion profiles in sporadic Creutzfeldt-Jakob disease. Neurology. 2009; 72(23): 1994-2001.

Meissner M, Dechat T, Gerner C, Grimm R, Foisner R, Sauermann G. Differential nuclear localization and nuclear matrix association of the splicing factors PSF and PTB. J Cell Biochem. 2000; 76(4): 559-566.

Mejía S, Giraldo M, Pineda D, Ardila A, Lopera F. Nongenetic factors as modifiers of the age of onset of familial Alzheimer's disease. Int Psychogeriatr. 2003; 15(4): 337-349.

Melton AA, Jackson J, Wang J, Lynch KW. Combinatorial control of signal-induced exon repression by hnRNPL and PSF. Mol Cell Biol. 2007; 27(19): 69726984.

Michelitsch MD, Weissman JS. A census of glutamine/asparagine-rich regions: implications for their conserved function and the prediction of novel prions. Proc Natl Acad Sci USA. 2000; 97(22): 11910-11915. 
Mirra SS, Heyman A, McKeel D et al. The Consortium to Establish a Registry for Alzheimer's Disease (CERAD). Part II. Standardization of the neuropathologic assessment of Alzheimer's disease. Neurology. 1991; 41(4): 479-486.

Molliex A, Temirov J, Lee J, Coughlin M, Kanagaraj AP, Kim HJ, Mittag T, Taylor JP. Phase separation by low complexity domains promotes stress granule assembly and drives pathological fibrillization. Cell. 2015; 163(1): 123-133.

Montine TJ, Phelps CH, Beach TG, Bigio EH, Cairns NJ, Dickson DW, Duyckaerts C, Frosch MP, Masliah E, Mirra SS, Nelson PT, Schneider JA, Thal DR, Trojanowski JQ, Vinters HV, Hyman BT; National Institute on Aging; Alzheimer's Association. National Institute on Aging-Alzheimer's Association guidelines for the neuropathologic assessment of Alzheimer's disease: a practical approach. Acta Neuropathol. 2012; 123(1): 1-11.

Mori F, Tanji K, Toyoshima Y, Sasaki H, Yoshida M, Kakita A, Takahashi H, Wakabayashi K. Valosin-containing protein immunoreactivity in tauopathies, synucleinopathies, polyglutamine diseases and intranuclear inclusion body disease. Neuropathology. 2013; 33(6): 637-644.

Nelson PT, Braak H, Markesbery WR. Neuropathology and cognitive impairment in Alzheimer disease: a complex but coherent relationship. $J$ Neuropathol Exp Neurol. 2009; 68(1): 1-14.

Nesvizhskii Al, Keller A, Kolker E, Aebersold R. A statistical model for identifying proteins by tandem mass spectrometry. Anal. Chem. 2003; 75(17): 4646-4658.

Neumann M, Sampathu DM, Kwong LK, Truax AC, Micsenyi MC, Chou TT, Bruce J, Schuck T, Grossman M, Clark CM, McCluskey LF, Miller BL, Masliah E, Mackenzie IR, Feldman H, Feiden W, Kretzschmar HA, Trojanowski JQ, Lee VM. Ubiquitinated TDP-43 in frontotemporal lobar degeneration and amyotrophic lateral sclerosis. Science. 2006; 314(5796): 130-133. 
Niedzielska E, Smaga I, Gawlik M, Moniczewski A, Stankowicz P, Pera J, Filip M. Oxidative stress in neurodegenerative diseases. Mol Neurobiol. 2016; 53(6): 4094-4125.

Nussbacher JK, Batra R, Lagier-Tourenne C, Yeo GW. RNA-binding proteins in neurodegeneration: Seq and you shall receive. Trends Neurosci. 2015; 38(4): 226-236.

Oddo S, Caccamo A, Shepherd JD, Murphy MP, Golde TE, Kayed R, Metherate R, Mattson MP, Akbari Y, LaFerla FM. Triple-transgenic model of Alzheimer's disease with plaques and tangles: intracellular $\mathrm{A} \beta \mathrm{a}$ and synaptic dysfunction. Neuron. 2003; 39(3): 409-421.

Otto H, Dreger M, Bengtsson L, Hucho F. Identification of tyrosine-phosphorylated proteins associated with the nuclear envelope. Eur J Biochem. 2001; 268(2): 420-428.

Ownby RL, Crocco E, Acevedo A, John V, Loewenstein D. Depression and risk for Alzheimer disease: systematic review, meta-analysis, and metaregression analysis. Arch Gen Psychiatry. 2006; 63(5): 530-538.

Papasozomenos SC. Heat shock induces rapid dephosphorylation of tau in both female and male rats followed by hyperphosphorylation only in female rats: implications for Alzheimer's disease. J Neurochem. 1996; 66(3): 1140-1149.

Parchi P, Giese A, Capellari S, Brown P, Schulz-Schaeffer W, Windl O, Zerr I, Budka H, Kopp N, Piccardo P, Poser S, Rojiani A, Streichemberger N, Julien J, Vital C, Ghetti B, Gambetti P, Kretzschmar H. Classification of sporadic Creutzfeldt-Jakob disease based on molecular and phenotypic analysis of 300 subjects. Ann Neurol. 1999; 46(2): 224-233.

Parchi P, Strammiello R, Notari S, Giese A, Langeveld JP, Ladogana A, Zerr I, Roncaroli F, Cras P, Ghetti B, Pocchiari M, Kretzschmar H, Capellari S. Incidence and spectrum of sporadic Creutzfeldt-Jakob disease variants with mixed phenotype and co-occurrence of PrPSc types: an updated classification. Acta Neuropathol. 2009; 118(5): 659-671. 
Patten DA, Germain M, Kelly MA, Slack RS. Reactive oxygen species: stuck in the middle of neurodegeneration. J Alzheimers Dis. 2010; 20 Suppl 2: S357367.

Perl DP. Neuropathology of Alzheimer's disease. Mt Sinai J Med. 2010; 77(1): 32-42.

Petti E, Buemi V, Zappone A, Schillaci O, Broccia PV, Dinami R, Matteoni S, Benetti R, Schoeftner S. SFPQ and NONO suppress RNA: DNA-hybrid-related telomere instability. Nat Commun. 2019; 10(1): 1001.

Pickering BM, Willis AE. The implications of structured $5^{\prime}$ untranslated regions on translation and disease. Semin Cell Dev Biol. 2005; 16(1): 39-47.

Planel E, Miyasaka T, Launey T, Chui DH, Tanemura K, Sato S, Murayama O, Ishiguro K, Tatebayashi Y, Takashima A. Alterations in glucose metabolism induce hypothermia leading to tau hyperphosphorylation through differential inhibition of kinase and phosphatase activities: implications for Alzheimer's disease. J Neurosci. 2004; 24(10): 2401-2411.

Planel E, Yasutake K, Fujita SC, Ishiguro K. Inhibition of protein phosphatase 2A overrides tau protein kinase $1 /$ glycogen synthase kinase 3 beta and cyclindependent kinase 5 inhibition and results in tau hyperphosphorylation in the hippocampus of starved mouse. J Biol Chem. 2001; 276(36): 34298-34306.

Prilusky J, Felder CE, Zeev-Ben-Mordehai T, Rydberg EH, Man O, Beckmann JS, Silman I, Sussman JL. Foldlndex: a simple tool to predict whether a given protein sequence is intrinsically unfolded. Bioinformatics. 2005; 21(16): 3435-3438.

Prince M, Bryce R, Albanese E, Wimo A, Ribeiro W, Ferri CP. The global prevalence of dementia: a systematic review and metaanalysis. Alzheimers Dement. 2013; 9(1): 63-75.

Prince MJ, Wimo A, Guerchet MM, Ali GC, Wu Y-T, Prina M. World Alzheimer report 2015 - The global impact of dementia: an analysis of prevalence, incidence, cost and trends. London: Alzheimer's disease international, 2015. 84 p. 
Prusiner SB. Novel proteinaceous infectious particles cause scrapie. Science. 1982; 216(4542): 136-144.

Ramaswami M, Taylor JP, Parker R. Altered ribostasis: RNA-protein granules in degenerative disorders. Cell. 2013; 154(4): 727-736.

Rangaraju V, Tom DS, Schuman EM. Local translation in neuronal compartments: how local is local? EMBO Rep. 2017; 18(5): 693-711.

Reitz C, Mayeux R. Alzheimer disease: epidemiology, diagnostic criteria, risk factors and biomarkers. Biochem Pharmacol. 2014; 88(4): 640-651.

Reitz C. Alzheimer's disease and the amyloid cascade hypothesis: a critical review. Int J Alzheimers Dis. 2012; 2012: 369808.

Ren Y, Sahara N. Characteristics of tau oligomers. Front Neurol. 2013; 4: 102.

Resende R, Moreira PI, Proenca T, Deshpande A, Busciglio J, Pereira C, Oliveira CR. Brain oxidative stress in a triple-transgenic mouse model of Alzheimer disease. Free Radic Biol Med. 2008; 44(12): 2051-2057.

Riback JA, Katanski CD, Kear-Scott JL, Pilipenko EV, Rojek AE, Sosnick TR, Drummond DA. Stress-triggered phase separation is an adaptive, evolutionarily tuned response. Cell. 2017; 168(6): 1028-1040.

Richter JD, Klann E. Making synaptic plasticity and memory last: mechanisms of translational regulation. Genes Dev. 2009; 23(1): 1-11.

Rossi G, Conconi D, Panzeri E, Redaelli S, Piccoli E, Paoletta L, Dalpra L, Tagliavini F. Mutations in MAPT gene cause chromosome instability and introduce copy number variations widely in the genome. J Alzheimers Dis. 2013; 33(4): 969-982.

Rossi G, Dalpra L, Crosti F, Lissoni S, Sciacca FL, Catania M, Di FG, Mangieri M, Giaccone G, Croci D, Tagliavini F. A new function of microtubule-associated protein tau: involvement in chromosome stability. Cell Cycle. 2008; 7(12): 1788-1794. 
Sabate R, Rousseau F, Schymkowitz J, Batlle C, Ventura S. Amyloids or prions? That is the question. Prion. 2015; 9(3): 200-206.

Sabate R, Rousseau F, Schymkowitz J, Ventura S. What makes a protein sequence a prion? PLoS Comput Biol. 2015; 11(1): e1004013.

Sanabria-Castro A, Alvarado- Echeverría I, Monge-Bonilla C. Molecular pathogenesis of Alzheimer's disease: an update. Ann Neurosci. 2017; 24(1): 46-54.

Sanchez-Mut JV, Graff J. Epigenetic alterations in Alzheimer's disease. Front Behav Neurosci. 2015; 9: 347.

Santacruz K, Lewis J, Spires T, Paulson J, Kotilinek L, Ingelsson M, Guimaraes A, DeTure M, Ramsden M, McGowan E, Forster C, Yue M, Orne J, Janus C, Mariash A, Kuskowski M, Hyman B, Hutton M, Ashe KH. Tau suppression in a neurodegenerative mouse model improves memory function. Science. 2005; 309(5733): 476-481.

Sardiu ME, Florens L, Washburn MP. Evaluation of clustering algorithms for protein complex and protein interaction network assembly. J Proteome Res. 2009; 8(6): 2944-2952.

Scheltens P, Blennow K, Breteler MM, De SB, Frisoni GB, Salloway S, Van der Flier WM. Alzheimer's disease. Lancet. 2016; 388(10043): 505-517.

Schmidt C, Redyk K, Meissner B, Krack L, von Ahsen N, Roeber S, Kretzschmar H, Zerr I. Clinical features of rapidly progressive Alzheimer's disease. Dement Geriatr Cogn Disord. 2010; 29(4): 371-378.

Schmidt C, Wolff M, Weitz M, Bartlau T, Korth C, Zerr I. Rapidly progressive Alzheimer disease. JAMA Neurology. 2011; 68(9): 1124-1130.

Selkoe DJ, Hardy J. The amyloid hypothesis of Alzheimer's disease at 25 years. EMBO Mol Med. 2016; 8(6): 595-608. 
Sellier C, Usdin K, Pastori C, Peschansky VJ, Tassone F, Charlet-Berguerand N. The multiple molecular facets of fragile $\mathrm{X}$-associated tremor/ataxia syndrome. J Neurodev Disord. 2014; 6(1): 23.

Sengupta U, Montalbano M, McAllen S, Minuesa G, Kharas M, Kayed R. Formation of toxic oligomeric assemblies of RNA-binding protein: musashi in Alzheimer's disease. Acta Neuropathol Commun. 2018; 6(1): 113.

Sephton CF, Yu G. The function of RNA-binding proteins at the synapse: implications for neurodegeneration. Cell Mol Life Sci. 2015; 72(19): 36213635.

Shafiei SS, Guerrero-Muñoz MJ, Castillo-Carranza DL. Tau oligomers: cytotoxicity, propagation, and mitochondrial damage. Front Aging Neurosci. 2017; 9: 83.

Sharathchandra A, Lal R, Khan D, Das S. Annexin A2 and PSF proteins interact with p53 IRES and regulate translation of p53 mRNA. RNA Biol. 2012; 9(12): 1429-1439.

Shav-Tal Y, Cohen M, Lapter S, Dye B, Patton JG, Vandekerckhove J, Zipori D. Nuclear relocalization of the pre-mRNA splicing factor PSF during apoptosis involves hyperphosphorylation, masking of antigenic epitopes, and changes in protein interactions. Mol Biol Cell. 2001; 12(8): 2328-2340.

Shchepachev V, Bresson S, Spanos C, Petfalski E, Fischer L, Rappsilber J, Tollervey D. Defining the RNA interactome by total RNA-associated protein purification. Mol Syst Biol. 2019; 15(4): e8689.

Shea TB, Cressman CM. A 26-30 kDa developmentally-regulated tau isoform localized within nuclei of mitotic human neuroblastoma cells. Int $J$ Dev Neurosci. 1998; 16(1): 41-48.

Siano G, Varisco M, Caiazza MC, Quercioli V, Mainardi M, Ippolito C, Cattaneo A, Primio CD: Nuclear tau modulates VGluT1 expression: a new function for tau. J Mol Biol. 2019; 431(4): 873-884. 
Sjöberg MK, Shestakova E, Mansuroglu Z, Maccioni RB, Bonnefoy E. Tau protein binds to pericentromeric DNA: a putative role for nuclear tau in nucleolar organization. J Cell Sci. 2006; 119(10): 2025-2034.

Slomnicki LP, Pietrzak M, Vashishta A, Jones J, Lynch N, Elliot S, Poulos E, Malicote D, Morris BE, Hallgren J, Hetman M. Requirement of neuronal ribosome synthesis for growth and maintenance of the dendritic tree. J Biol Chem. 2016; 291(11): 5721-5739.

Soto ME, Andrieu S, Arbus C, Ceccaldi M, Couratier P, Dantoine T, Dartigues JF, Gillette-Guyonnet S, Nourhashemi F, Ousset PJ, Poncet M, Portet F, Touchon J, Vellas B. Rapid cognitive decline in Alzheimer's disease. Consensus paper. J Nutr Health Aging. 2008; 12(10): 703-713.

Stoeck K, Sanchez-Juan P, Gawinecka J, Green A, Ladogana A, Pocchiari M, Sanchez-Valle R, Mitrova E, Sklaviadis T, Kulczycki J, Slivarichova D, Saiz A, Calero M, Knight R, Aguzzi A, Laplanche JL, Peoc'h K, Schelzke G, Karch A, van Duijn CM, Zerr I. Cerebrospinal fluid biomarker supported diagnosis of Creutzfeldt-Jakob disease and rapid dementias: a longitudinal multicentre study over 10 years. Brain. 2012; 135(Pt 10): 3051-3061.

Stoeck K, Schmitz M, Ebert E, Schmidt C, Zerr I. Immune responses in rapidly progressive dementia: a comparative study of neuroinflammatory markers in Creutzfeldt-Jakob disease, Alzheimer's disease and multiple sclerosis. $J$ Neuroinflammation. 2014; 11: 170.

Su B, Wang X, Lee HG, Tabaton M, Perry G, Smith MA, Zhu X. Chronic oxidative stress causes increased tau phosphorylation in M17 neuroblastoma cells. Neurosci Lett. 2010; 468(3): 267-271.

Sultan A, Nesslany F, Violet M, Begard S, Loyens A, Talahari S, Mansuroglu Z, Marzin D, Sergeant N, Humez S, Colin M, Bonnefoy E, Buee L, Galas MC: Nuclear tau, a key player in neuronal DNA protection. J Biol Chem. 2011; 286(6): 4566-4575. 
Suzuki K, Bose P, Leong-Quong RY, Fujita DJ, Riabowol K. REAP: A two minute cell fractionation method. BMC Res Notes. 2010; 3: 294.

Tanzi RE, Bertram L. Twenty years of the Alzheimer's disease amyloid hypothesis: a genetic perspective. Cell. 2005; 120(4): 545-55.

Tarawneh R, Holtzman DM. The clinical problem of symptomatic Alzheimer disease and mild cognitive impairment. Cold Spring Harb Perspect Med. 2012; 2(5): a006148.

Tassone F, Iwahashi C, Hagerman PJ. FMR1 RNA within the intranuclear inclusions of fragile X-associated tremor/ataxia syndrome (FXTAS). RNA Biol. 2004; 1(2): 103-105.

Thal DR, Rüb U, Orantes M, Braak H. Phases of Abeta-deposition in the human brain and its relevance for the development of AD. Neurology. 2002; 58(12): 1791-800.

Toombs JA, McCarty BR, Ross ED. Compositional determinants of prion formation in yeast. Mol Cell Biol. 2010; 30(1): 319-332.

Toombs JA, Petri M, Paul KR, Kan GY, Ben-Hur A, Ross ED. De novo design of synthetic prion domains. Proc Natl Acad Sci USA. 2012; 109(17): 65196524.

Toretsky JA, Wright PE2. Assemblages: functional units formed by cellular phase separation. Cell Biol. 2014; 206(5): 579-88.

Tosto G, Gasparini M, Brickman AM, Letteri F, Renie' R, Piscopo P, Talarico G, Canevelli M, Confaloni A, Bruno G. Neuropsychological predictors of rapidly progressive Alzheimer's disease. Acta Neurol Scand. 2015; 132(6): 417422.

Tschampa HJ, Kallenberg K, Kretzschmar HA, Meissner B, Knauth M, Urbach H, Zerr I. Pattern of cortical changes in sporadic Creutzfeldt-Jakob disease. AJNR Am J Neuroradiol. 2007; 28(6): 1114-1118. 
Turakhiya A, Meyer SR, Marincola G, Bohm S, Vanselow JT, Schlosser A, Hofmann K, Buchberger A. ZFAND1 recruits p97 and the 26S proteasome to promote the clearance of arsenite-induced stress granules. Mol Cell. 2018; 70(5): 906-919.

Tyanova S, Cox J. Perseus: a bioinformatics platform for integrative analysis of proteomics data in cancer research. Methods Mol Biol. 2018; 1711: 133148.

Tyedmers J, Treusch S, Dong J, McCaffery JM, Bevis B, Lindquist S. Prion induction involves an ancient system for the sequestration of aggregated proteins and heritable changes in prion fragmentation. Proc Natl Acad Sci USA. 2010; 107(19): 8633-8638.

Ulrich G, Salvadè A, Boersema P, Calì T, Foglieni C, Sola M, Picotti P, Papin S, Paganetti $\mathrm{P}$. Phosphorylation of nuclear tau is modulated by distinct cellular pathways. Sci Rep. 2018; 8(1): 17702.

Valverde R, Edwards L, Regan L. Structure and function of KH domains. FEBS J. 2008; 275(11): 2712-2726.

Vance C, Scotter EL, Nishimura AL, Troakes C, Mitchell JC, Kathe C, Urwin H, Manser C, Miller CC, Hortobagyi T, Dragunow M, Rogelj B, Shaw CE. ALS mutant FUS disrupts nuclear localization and sequesters wild-type FUS within cytoplasmic stress granules. Hum Mol Genet. 2013; 22(13): 26762688.

Vanderweyde T, Apicco DJ, Youmans-Kidder K, Ash PEA, Cook C, Lummertz da RE, Jansen-West K, Frame AA, Citro A, Leszyk JD, Ivanov P, Abisambra JF, Steffen M, Li H, Petrucelli L, Wolozin B. Interaction of tau with the RNAbinding rotein TIA1 regulates tau pathophysiology and toxicity. Cell Rep. 2016; 15(7): 1455-1466.

Vanderweyde T, Yu H, Varnum M, Liu-Yesucevitz L, Citro A, Ikezu T, Duff K, Wolozin B. Contrasting pathology of the stress granule proteins TIA-1 and G3BP in tauopathies. J Neurosci. 2012; 32(24): 8270-8283. 
Varadi M, Zsolyomi F, Guharoy M, Tompa P. Functional advantages of conserved intrinsic disorder in RNA-binding proteins. PLoS One. 2015; 10(10): e0139731.

Verdile V, De Paola E, Paronetto MP. Aberrant phase transitions: side sffects and novel therapeutic strategies in human disease. Front Genet. 2019; 10: 173.

Wahl MC, Will CL, Luhrmann R. The spliceosome: design principles of a dynamic RNP machine. Cell. 2009; 136(4): 701-718.

Wang C, Szaro BG. Post-transcriptional regulation mediated by specific neurofilament introns in vivo. J. Cell Sci. 2016; 129(7): 1500.

Wang X, Wang W, Li L, Perry G, Lee HG, Zhu X. Oxidative stress and mitochondrial dysfunction in Alzheimer's disease. Biochim Biophys Acta. 2014; 1842(8): 1240-1247.

Wang Y, Loomis PA, Zinkowski RP, Binder LI. A novel tau transcript in cultured human neuroblastoma cells expressing nuclear tau. J Cell Biol. 1993; 121(2): 257-267.

Wei Y, Qu MH, Wang XS, Chen L, Wang DL, Liu Y, Hua Q, He RQ. Binding to the minor groove of the double-strand, tau protein prevents DNA from damage by peroxidation. PLoS One. 2008; 3(7): e2600.

Weingarten MD, Lockwood AH, Hwo SY, Kirschner MW. A protein factor essential for microtubule assembly. Proc Natl Acad Sci USA. 1975; 72(5): 1858-1862.

Wickner RB, Shewmaker FP, Bateman DA, Edskes HK, Gorkovskiy A, Dayani Y, Bezsonov EE. Yeast prions: structure, biology, and prion-handling systems. Microbiol Mol Biol Rev. 2015; 79(1): 1-17.

Wolozin B: Regulated protein aggregation: stress granules and neurodegeneration. Mol Neurodegener. 2012; 7: 56.

Wright PE, Dyson HJ. Intrinsically disordered proteins in cellular signalling and regulation. Nat Rev Mol Cell Biol. 2015; 16(1): 18-29. 
Wurth L. Versatility of RNA-binding proteins in cancer. Comp Func Genomics. 2012; 2012: 178525.

Xing L, Bassell GJ. mRNA localization: an orchestration of assembly, traffic and synthesis. Traffic. 2013; 14(1): 2-14.

$\mathrm{Xu}$ J, Zhong N, Wang $\mathrm{H}$ et al. The Parkinson's disease-associated DJ-1 protein is a transcriptional co-activator that protects against neuronal apoptosis. Hum $\mathrm{Mol}$ Genet. 2005; 14(9): 1231-1241.

Yanagisawa M, Planel E, Ishiguro K, Fujita SC. Starvation induces tau hyperphosphorylation in mouse brain: implications for Alzheimer's disease. FEBS Lett. 1999; 461(3): 329-333.

Yarosh CA, lacona JR, Lutz CS, Lynch KW. PSF: nuclear busy-body or nuclear facilitator? Wiley Interdiscip Rev RNA. 2015; 6(4): 351-67.

Yasuda K, Clatterbuck-Soper SF, Jackrel ME, Shorter J, Mili S. FUS inclusions disrupt RNA localization by sequestering kinesin-1 and inhibiting microtubule detyrosination. J Cell Biol. 2017; 216(4): 1015-1034.

Yeo G, Holste D, Kreiman G, Burge CB. Variation in alternative splicing across human tissues. Genome Biol. 2004; 5(10): R74.

Zafar S, Shafiq M, Younas N, Schmitz M, Ferrer I, Zerr I. Prion protein interactome: identifying novel targets in slowly and rapidly progressive forms of Alzheimer's disease. J Alzheimers Dis. 2017; 59(1): 265-275.

Zafar S, Younas N, Sheikh N, Tahir W, Shafiq M, Schmitz M, Ferrer I, Andréoletti O, Zerr I. Cytoskeleton-associated risk modifiers involved in early and rapid progression of sporadic Creutzfeldt-Jakob disease. Mol Neurobiol. 2018; 55(5): 4009-4029.

Zambrano R, Conchillo-Sole O, Iglesias V, Illa R, Rousseau F, Schymkowitz J, Sabate $\mathrm{R}$, Daura $\mathrm{X}$, Ventura $\mathrm{S}$. PrionW: a server to identify proteins containing glutamine/asparagine rich prion-like domains and their amyloid cores. Nucleic Acids Res. 2015; 43(W1): W331-W337. 
Zerr I, Parchi P. Sporadic Creutzfeldt-Jakob disease. Handb Clin Neurol. 2018; 153: 155-174.

Zhang X, Poo MM. Localized synaptic potentiation by BDNF requires local protein synthesis in the developing axon. Neuron. 2002; 36(4): 675-688.

Zhang Z, Boonen K, Ferrari P, Schoofs L, Janssens E, van N, V, Rolland F, Geuten K. UV crosslinked mRNA-binding proteins captured from leaf mesophyll protoplasts. Plant Methods. 2016; 12: 42.

Zhou H, Mangelsdorf M, Liu J, Zhu L, Wu JY. RNA-binding proteins in neurological diseases. Sci China Life Sci. 2014; 57(4): 432-444.

Zhou Y, Dong F, Mao Y. Control of CNS functions by RNA-binding proteins in neurological diseases. Curr Pharmacol Rep. 2018; 4(4): 301-313.

Zhu X, Lee HG, Casadesus G, Avila J, Drew K, Perry G, Smith MA. Oxidative imbalance in Alzheimer's disease. Mol Neurobiol. 2005; 31(1-3): 205-217. 


\section{Acknowledgements}

First and foremost, I would like to thank "Allah Almighty" who has blessed and guided me so that I was able to accomplish this thesis. In addition, may peace and salutation be given to the prophet Muhammad (PBUH), who is forever a beacon of light and knowledge for me.

I would like to express my sincerest gratitude to Prof. Dr. med. Inga Zerr for all the support and confidence she gave me. I would like to thank her for encouraging my research approaches and for shaping my scientific attitude by her insightful discussions and inputs. I consider myself lucky to work with such a motivated and focused leader. I feel pleasure in acknowledging Dr. Saima Zafar, who has supported me throughout my research work. Her encouragement made this project exciting from the beginning on. Her good advice, support and friendship has been invaluable on both an academic and a personal level, for which I am extremely grateful to her.

I express profound gratitude to my thesis committee members, Prof. Dr. Thomas Meyer and Prof. Dr. Tiago Fleming Outeiro, for their supervision, support, encouragement and contribution to my work. Their suggestions are highly appreciated, and without whom this dissertation would not be completed. I owe a debt of gratitude and appreciation to Dr. Jesús R. Requena, University of Santiago de CompostelaSpain for referring me in Prion Lab, UMG. His support and encouragement meant a great deal to me. I would also like to thank, Dr. Nadeem Sheikh for his invaluable support and guidance since my bachelor thesis. I present my sincere thanks to Dr. Waqas Tahir for his assistance and guidance from very first day in the lab, and I admire his helping nature.

In my daily work I have been blessed with a friendly and cheerful group. I am deeply thankful to Dr. Mohsin Shafiq, who was always willing to help and give his best suggestions. I present my thanks to other colleagues and friends of the prion research lab, Dr. Amandeep, Dr. Matthias Schmitz, Dr. Katrin Thüne, Dr. Niccolo, Dr. Katrin Ditmar, Dr. Maria, Aneeqa, Tobias, Anna S., Anna L., Susana, Angela, Vivi and Mandy for providing a good work environment, and for their direct or indirect help. I am thankful to Dr. Christof Lenz for his inputs in the bioinformatics analysis. Great thanks to our secretaries Maja and Jolanthe. Without their ambitious work we would be lost in administrative issues.

Special thanks are due to my best, cute little friend, Arham for being such a good girl always cheering me up and sharing a great time in Göttingen. Her smiling face was always a source of cherishment and motivation for me. I would like to say a heartfelt thank you to my dearest friend Dr. Sidra Shahid for her endless support, love, and encouragement throughout the process. I'll never forget the many wonderful adventures and fun activities we've done together. I know that when we are old, Sidra will still be there as a supportive and caring friend. I would also like to thank Kiran for her love and constant support. Many thanks to Maryam for our shared laughter, support and encouragement. Special word of thank to my office neighbour, Miss Homburg for her cheerful chit chat and moral support.

Last, but by no means least, I would like to thank my family back home, my sister Swaiba, brother Asrar, Amma Abba, and Uncle Zulfiqar, for their love, prayers and support. Special thanks to my brother Abrar for always believing in me and encouraging me to follow my dreams.

Neelam Younas 


\section{Biography}

Neelam Younas was born in 1991 in Sialkot, Pakistan. She received her higher secondary education with "role of honor (Gold medal)" from Government Girls college, Pasrur, Pakistan. She received her bachelor's degree in Zoology (2012) with "role of honor (Gold medal)" from University of the Punjab, Lahore, Pakistan. Following her graduation, Neelam got a joint Master's degree with "role of honor (First position)" in Zoology (2014) from the University of Santiago de Compostela, Spain, and the University of the Punjab, Pakistan. For her Master's thesis, she joined the Prion Research Lab at the Department of Neurology, University Medical Centre Göttingen, Germany. She continued her research for a PhD project (October, $2015)$ in the same laboratory with the thesis title "Emerging role of RNA-binding proteins in sporadic and rapid progressive Alzheimer's disease". Neelam was involved in research projects investigating the molecular basis and risk factors of neurodegenerative diseases, including Alzheimer's and prion diseases. She has published following papers,

- Younas N., Zafar S., Sheikh N., Tahir W., Shafiq M., Schmitz M., Grewe, V., Ferrer I., Andréoletti O., Zerr I. Cytoskeleton-associated risk modifiers involved in early and rapid progression of sporadic Creutzfeldt-Jakob disease. Mol. Neurobiol. 2017; 55(5): 4009-4029.

- Sevillano A, M., Fernández-Borges, N., Younas, N., Wang, F., R Elezgarai S, Bravo S, Vázquez-Fernández E, Rosa I, Eraña H2, Gil D, Veiga S, Vidal E, Erickson-Beltran ML, Guitián E, Silva CJ, Nonno R, Ma J, Castilla J, R Requena J. Recombinant PrPSc shares structural features with brain-derived PrPSc: Insights from limited proteolysis. PLoS Pathog. 2018; 14(1): e1006797.

- Zafar, S., Shafiq, M., Younas, N., Schmitz, M., Ferrer, I., Zerr, I. Prion protein interactome: identifying novel targets in slowly and rapidly progressive forms of Alzheimer's disease. J Alzheimers Dis. 2017; 59(1): 265-275.

- Zafar S, Younas N, Zerr I. 2017. Subtype specific CSF biomarkers in sporadic Creutzfeldt-Jakob disease. J Alzheimer's Dis Parkinsonism. 2017; 7: 332.

- Zafar, S., Younas, N., Correia, S., Shafiq, M., Tahir, W., Schmitz, M., Andéoletti, O., Zerr, I., 2016. Strain-specific altered regulatory response of Rab7a and tau in Creutzfeldt-Jakob disease and Alzheimer's disease. Mol. Neurobiol. 54(1): 697-709.

- Zafar, S., Younas, N., Zerr, I., 2016. Novel biomarkers and the diagnosis of prion diseases. J Mol Biomarkers Diagn. S8: 011.

- Zafar, S., Schmitz, M., Younas, N., Tahir, W., Shafiq, M., Llorens, F., Ferrer, I. Andéoletti, O., Zerr, I. 2015. Creutzfeldt-Jakob disease subtype-specific regional and temporal regulation of ADP ribosylation factor-1-dependent Rho/MLC pathway at pre-clinical stage. J Mol Neurosci. 56(2): 329-48. 